BNL- 66931

Informal Report

\title{
AGS Experiment 945A Radiation Damage in Metals at Liquid Helium Temperature by $\mathrm{GeV}$ Protons
}

\author{
G.A. Greene, C.C. Finfrock, C.L. Snead and A.L. Hanson \\ Brookhaven National Laboratory \\ and \\ W.F. Sommer and M.R. James \\ Los Alamos National Laboratory
}

August 1999

Department of Advanced Technology

Brookhaven National Laboratory

Upton, Long Island, New York 11973 


\title{
AGS Experiment 945A \\ Radiation Damage in Metals at Liquid Helium Temperature by GeV Protons*
}

\author{
G.A. Greene, C.C. Finfrock, C.L. Snead and A.L. Hanson \\ Brookhaven National Laboratory \\ and \\ W.F. Sommer and M.R. James \\ Los Alamos National Laboratory
}

August 1999

Department of Advanced Technology

Brookhaven National Laboratory

Brookhaven Science Associates

Upton, Long Island, New York 11973

Under Contract No. DE-AC02-98CH10886

UNITED STATES DEPARTMENT OF ENERGY

*This work was performed under the auspices of the U.S. Department of Energy 


\begin{abstract}
The purpose of this experiment was to provide data for the validation and improvement of models used in computer codes which are being used to predict radiation-induced damage in target and blanket materials of APT and similar technologies when the materials are irradiated with GeVrange protons. The experiment was performed at the BNL AGS at liquid helium temperatures, employing a commercially-available flow cryostat for the mounting of the samples. The shaft of the cryostat is mounted on a 2.75 -inch conflat flange around which the vacuum chamber was constructed. The target chamber of the vacuum vessel was a six-inch diameter six-way cross. Two flanges on this cross were for the 0.010-inch beam windows; a third flange was for the cryostat mount; a fourth flange was for the vacuum valve and turbo-roughing pump assembly; a fifth flange was for the vacuum gauge assembly, and the sixth flange was for the electrical fecdthrough for the instrumentation wires to the data-acquisition system. Target samples were prepared from copper and tungsten wire and mounted to the cryostat tip. Each sample consisted of 0.002 -inch diameter wire, wrapped around U-shaped posts, and anchored to OFHC copper bases. The U-shaped posts were made of anodized aluminum threaded rods which insulated the wires electrically while providing good thermal contact to the hase. The radiation damage was determined indirectly by measuring changes in the resistivities of the samples using the four-wire current reversal technique. The temperature of the target assembly was monitored during the experiments by a Cernox ${ }^{\mathrm{TM}}$ model CX1070-AA-4L resistor attached to the copper base. The data-acquisition instrumentation used to measure the resistance changes of the sample wires was also used to measure the resistance of the Cernox ${ }^{\mathrm{TM}}$ resistor as well. A series of experiments were performed with both 1.94-GeV and 1.10$\mathrm{GeV}$ protons with the sample wires at $4.7 \mathrm{~K}$ to "freeze" the radiation-induced defects into the samples in order to measure the defect-induced changes in electrical resistivity.

Resistance changes due to point-defect production were measured to be on the order of 2-3 $\mu \Omega / \mathrm{TP}_{\mathrm{i}}$ for copper and $60-90 \mu \Omega / \mathrm{TP}_{\mathrm{i}}$ for tungsten, easily measurable with the present instrumentation $\left(\mathrm{TP}=10^{12}\right.$ protons incident on target). Activation foils and an integrating current transformer were used to measure the total proton fluence for each experiment, and beam spot size estimates were made from analyses of the activity distributions of the activation foils and the videotapes of the beam line phosphor upstream of the target. The proton beam spot diameters so estimated were $1.6 \mathrm{~cm}$ for the $1.94-\mathrm{GeV}$ experiments and $2.4 \mathrm{~cm}$ for the $1.10-\mathrm{GeV}$ experiments. An engineering analysis of the experimental data was performed to convert the raw experimental measurements into measured electrical resistivity increases of the samples in order to compare to calculations of the predicted resistivity increases of the samples due to defect production in the wire samples. The measured resistivity increases for both copper and tungsten were found to compare well with predicted resistivity increases that were calculated from radiation damage parameters in the literature. The ratios of the measured-to-calculated resistivity incrcascs vs. the measured resistivity increases were calculated for each of the thirteen data points. The tungsten ratio was nearly unity for the $1.94-\mathrm{GeV}$ proton data and fell to 0.78 for the $1.10-\mathrm{GeV}$ data. The copper ratios were not as good, ranging from 0.62 for the $1.94-\mathrm{GeV}$-proton data to 0.57 for the $1.10-\mathrm{GeV}$ data. The predictions always exceeded the measured resistivity increases; the comparisons improved with increasing atomic number of the sample and with increasing proton energy over the range of atomic numbers and proton energies investigated.
\end{abstract}




\section{TABLE OF CONTENTS}

Page

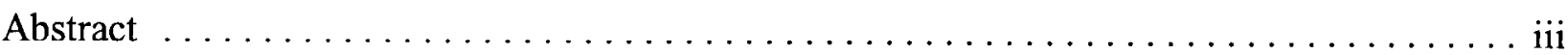

List of Figures and Tables $\ldots \ldots \ldots \ldots \ldots \ldots \ldots \ldots \ldots \ldots \ldots \ldots \ldots \ldots$ vii

Background and Objectives $\ldots \ldots \ldots \ldots \ldots \ldots \ldots \ldots \ldots \ldots \ldots \ldots \ldots \ldots$

Description of Cryostat Assembly $\ldots \ldots \ldots \ldots \ldots \ldots \ldots \ldots \ldots \ldots \ldots \ldots \ldots \ldots$

Description of Vacuum Chamber $\ldots \ldots \ldots \ldots \ldots \ldots \ldots \ldots \ldots \ldots \ldots \ldots \ldots$

Description of Test Samples and Temperature Sensor $\ldots \ldots \ldots \ldots \ldots \ldots \ldots \ldots \ldots$

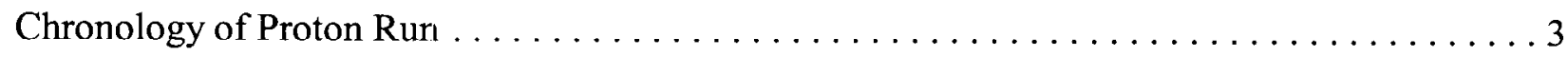

Discussion of Test Results $\ldots \ldots \ldots \ldots \ldots \ldots \ldots \ldots \ldots \ldots \ldots \ldots \ldots \ldots \ldots$

Preliminary Data Analyses and Observations $\ldots \ldots \ldots \ldots \ldots \ldots \ldots \ldots \ldots \ldots \ldots \ldots \ldots \ldots$

Contents of Appendices $\ldots \ldots \ldots \ldots \ldots \ldots \ldots \ldots \ldots \ldots \ldots \ldots \ldots \ldots \ldots$

Appendix 1: Experiment Run Sheets $\ldots \ldots \ldots \ldots \ldots \ldots \ldots \ldots \ldots \ldots \ldots \ldots \ldots$

Appendix 2: EXCEL Data File of Resistance Measurements of Copper Sample \#1 . . . . 2.1

Appendix 3: EXCEL Data File of Resistance Measurements of Copper Sample \#2 . . . . 3.1

Appendix 4: EXCEL Data File of Resistance Measurements of Tungsten Samplc $\ldots . \ldots 4.1$ 


\section{LIST OF TABLES}

Page

Table $1 \quad$ Results of ${ }^{\prime}$ Total Proton Dosimetry for Experiment 945 A . . . . . . . . . 4

Table 2 EXCEL Spreadsheet for preliminary Engineering Analysis of Experimental Data from AGS Experiment 945A: Radiation Damage in Metals at Liquid

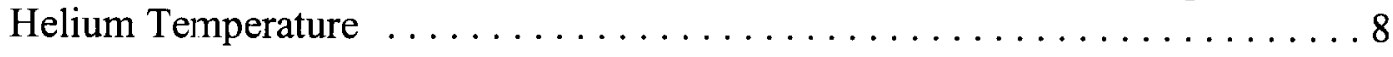

\section{LIST OF FIGURES}

Figure 1 Comparison of the Measured Resistivity Increases of the Wire Samples to the Calculated Increases Due to Proton Irradiation Based Upon Measurement of ${ }^{24} \mathrm{Na}$ Activity in Aluminum Activation Foils . . . . . . . . . . . . 5

Figure 2 Comparison of the Ratio of the Measured Resistivity Increases of the Wire Samples to the Calculated Increases Due to Proton Irradiation vs. Measured Resistivity Increase Based Upon Measurement of ${ }^{24} \mathrm{Na}$ Activity in Aluminum

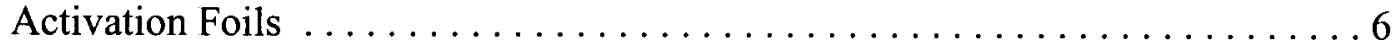




\section{BACKGROUND AND OBJECTIVES}

The purpose of this report is to provide a brief description of AGS Experiment 945A which was conducted to provide data for the validation and improvement of models used in computer codes which are being used to predict proton radiation damage in APT materials and to document and distribute the experimental data that was generated during the experiment. The experimental data have been made available to participants in a collaboration of several organizations who will work together to analyze the experimental data, perform coordinated Monte Carlo and molecular dynamics

simulations of the experirnents, determine the most pressing needs for model development and improvement, and generally advance the state-of-the-art of radiation damage simulations, both experimentally and calculationally, for application to the needs of the spallation physics community in general and the APT project in particular.

\section{DESCRIPTION OF CRYOSTAT ASSEMBLY}

A cryostat assembly for the cryogenic radiation-damage experiment at the BNL AGS was designed and assembled, employing a commercially-available flow cryostat for the mounting of the sample holder assembled at LANL. The cryostat can be used as a double-pipe liquid-nitrogen or liquid-helium flow system to deliver a metered flow of a liquid cryogen to an oxygen-free highconductivity $(\mathrm{OFHC})$ copper mounting surface onto which a test sample can be attached. The endmount is 0.75 -inch diameter and has a 1/4-28 threaded end to mount a test fixture. A thermal shield was mounted around the end of the cryostat to reflect incoming thermal radiation in order to achieve temperatures below $10 \mathrm{~K}$. The cryostat is 24 -inches long, accepts a standard screw-on capillary tube transfer line and has a heated exhaust to vent the boil-off gas. The transfer line and the cryostat are matched. The transfer line has a standard siphon bayonet on one end and a product supply bayonet on the other end to deliver the cryogen directly to the inside face of the OFHC mount at the end of the cryostat where the samples are mounted. The vapor returns in the annulus formed between the outer surface of the supply bayonet and the inside surface of the cryostat, offering additional thermal ballast to the liquid cryogen. The helium gas exits through the heated vent, and is directed through a calibrated gas flow meter to monitor and control the product supply flow rate. The transfer line has a vacuum jacket with super insulation, inside of which are two capillary tubes; one is for cryogen delivery to the cryostat and the other is to supply liquid helium to surround and thermally insulate the supply capillary. This is called the shroud flow. The shroud flow is exhausted and metered through a gas flow meter identical to the one which meters the cryostat exhaust. The transfer line is equipped with two non adjustable pressure relief valves to protect against over pressurization of the transfer line and the cryostat. The cryostat can operate continuously for more than 13 hours from a 60-liter helium dewar.

\section{DESCRIPTION OF VACUUM CHAMBER}

The shaft of the cryostat is provided with a 2.75-inch conflat flange, around which a vacuum chamber was constructed. A vacuum bellows provided for fine alignment adjustment of the cryostat 
inside of, and relative to, the vacuum chamber in order to position a target in the proton beam. The entire vacuum chamber was constructed with UHV conflat components. The business end of the vacuum chamber was a six-inch diameter six-way cross. Two flanges on this cross were for the 0.010 -inch beam windows; a third flange was for the cryostat mount; a fourth flange was for the vacuum valve and turbo-roughing pump assembly; a fifth flange was for the vacuum gauge assembly, and the sixth flange was for the electrical feedthrough for the instrumentation wires to the data-acquisition system. A.fter the cryostat assembly for the cryogenic radiation-damage experiment had been fully instrumented, a full simulation test was performed under a vacuum with flowing liquid helium. The vacuurn chamber could pump down to $10^{-8}$ Torr at room temperature, and could achieve $10^{-9}$ Torr with liquid helium flowing and the vacuum chamber isolated from the turbomolecular pump. The temperature at the surface of the OFHC mounting block at the tip of the cryostat was measured to be $4.3 \mathrm{~K}$ and the temperature in the base of the sample holder was measured to be $4.7 \mathrm{~K}$.

\section{DESCRIPTION OF TEST SAMPLES AND TEMPERATURE SENSOR}

The target wire sarnples $(\mathrm{Cu}, \mathrm{Al}, \mathrm{W})$ were prepared at BNL by LANL staff and mounted to the cryostat tip. Each samplc consisted of 0.002-inch diameter wire, wrapped around U-shaped posts, and anchored to OFHC copper bases with Stycast ${ }^{\mathrm{TM}}$ epoxy. The U-shaped posts were made of anodized aluminum threaded rods ( 80 threads per inch) which insulated the wires electrically while providing good thermal contact to the base. The wire was wrapped on every third thread on the posts. The wire wraps were anchored to the threaded rods with Stycast ${ }^{\mathrm{TM}}$ epoxy as well. The ends of the tungsten, copper and aluminum wire samples were terminated mechanically to copper pigtails and potted in electrically-conductive epoxy. The copper pigtails were then soft soldered to the four-wire source and sense leads which connect to the instrumentation penetration and from there to the data-acquisition system. All three of the samples were installed on the cryostat and wired to the data-acquisition system for shakedown testing at both room temperature and at cryogenic temperatures. The aluminum sample was not successfully connected to the copper pigtails due to the extreme fragility of the aluminum wire itself and was removed. The electrical connections of the tungsten sample exhibited degradation after cooldown. However, the defective tungsten test sample was diagnosed and repaired in place. A second copper test sample was installed in the cryostat in place of the aluminum test sample which provided the experiment with three test samples for the experiment, two copper samples and one tungsten sample. During the experiments, the proton beam was focused so that the illumination area was entirely enveloped by the wire wraps, thus illuminating only a fraction of the total wire length but by the entire proton beam. The temperature of the target assembly was monitored during the experiments by a Cernox ${ }^{\mathrm{TM}}$ resistive sensor mounted in a hole which had been drilled in the OFHC copper base. The Cernox ${ }^{\mathrm{TM}}$ resistance thermometer was chosen for this application because of its superior thermal stability at cryogenic temperatures, its precise factory calibration, its thermal sensitivity at liquid helium temperatures, and its resistance to damage in high radiation environments. The data-acquisition instrumentation which was used to measure the resistance changes of the sample wires by a four-wire current-reversal technique was used to measure the resistance of the Cernox ${ }^{\mathrm{TM}}$ thermal resistor as well. 


\section{CHRONOLOGY OF PROTON RUN}

Installation of the radiation-damage apparatus and associated hardware into the AGS U-line began on $3 / 10 / 99$. The apparatus was surveyed in place and fixed to mechanical stops on the traversing rails; the accuracy of repositioning the assembly to the stops was measured to be 0.005 inch. Installation of all hardware and electrical cables to the trailer was completed on 3/12/99. After the SNS run was completed (3/13/99 to 3/15/99), access to the beam line was regained and a final dry run at room temperature was performed in the AGS U-line to verify that all systems were functioning properly after the installation. This was accomplished at 1630 hours on 3/15/99. Beam tuning at $1.94-\mathrm{GeV}$ was conducted through the night of 3/15/99 and beam was delivered on-target at 0725 hours on 3/16/99. Three one-hour irradiations were planned for this beam energy. However, the third irradiation at $1.94-\mathrm{GeV}$ was terminated early due to an upset condition in the liquid hclium delivery system. The cause of this upset was subsequently identified to be the result of debris in the bottom of the liquid helium dewar which migrated to the suction end of the transfer line, causing a sudden reduction in the total liquid helium supply flow rate to the transfer line shroud and the cryostat from four liters per hour to two liters per hour. In order to protect the test samples from possible damage as a result of undercooling, the third run at $1.94-\mathrm{GeV}$ was terminated after 13 minutes of beam at 1345 hours on $3 / 16 / 99$. The test samples had heated to between 7.2 to $7.7 \mathrm{~K}$ due to the reduction in liquid helium flow, causing the copper samples to partially anneal. Although the data from the two copper samples was of no value for this prematurely terminated 13-minute run as a result of their partial anneal, the tungsten sample was unaffected and data were taken on the tungsten sample prior to the anneal to room temperature. At the end of the $1.94-\mathrm{GeV}$ irradiation and prior to the start of the $1.10-\mathrm{GeV}$ irradiation, the specimens were warmed (annealed) to room temperature. The beam was tuned to $1.10-\mathrm{GeV}$, and the beam was delivered on-target at 1140 hours on $3 / 17 / 99$. The liquid helium dewar had been replaced with a fresh dewar and liquid helium supply to the cryostat proceeded without incident. Although three one-hour irradiations were also planned for this beam energy, only two irradiations were performed due to schedule constraints. The 1.10$\mathrm{GeV}$ irradiation was terminated at 1500 hours on 3/17/99.

\section{DISCUSSION OF TEST RESULTS}

A discussion of the test results follows. In general, the temperatures of the samples were maintained at $4.7 \mathrm{~K}$ and the test chamber maintained a vacuum in the $10^{-9}$ Torr range. At $1.94-\mathrm{GeV}$, the copper samples were irradiated twice (150 minutes of beam) and the tungsten sample was irradiated thrice (163 minutes of beam). At $1.10-\mathrm{GeV}$, all three samples were irradiated twice (135 minutes of beam). The resistance measurements were of high precision, accurate, and reproducible. Resistance changes duc to point-defect production were measured to be on the order of 2-3 $\mu \Omega / \mathrm{TP}_{\mathrm{i}}$ for copper and 60-90 $\mu \Omega / T P_{i}$ for tungsten, easily measurable with the present instrumentation (TP $=10^{12}$ protons incident on target). A comparison of the results of the total-proton dosimetry by both activation foil analysis and integrating beam current transformer output for each of the five irradiations is given in Table 1. The activation foils were analyzed for ${ }^{24} \mathrm{Na}$ at $\mathrm{BNL}$ and for ${ }^{22} \mathrm{Na}$ at LANL. The results of the two independent activation foil analyses are in excellent agreement; the results for total protons are identical to three significant figures for two of the foils $(5 \mathrm{~B}, 6 \mathrm{~B})$ and the 
results for the other three foils $(5 \mathrm{~A}, 5 \mathrm{C}, 6 \mathrm{~A})$ agree to within $1 \%$ for total protons. The Bergoz model ICT-122-070-20:1 integrating current transformer results for total protons for the two irradiations at $1.10-\mathrm{GeV}$ are within $2 \%$ on the high side of the activation foil results. Confidence in the proton fluence for the five irradiations is very high. The column labeled UXF3-GPM are the results from a integrating current transformer located in the beam line upstream of the Bergoz ${ }^{\mathrm{TM}}$ integrating current transformer; the differences between the UXF3-GPM and Bergoz ${ }^{\mathrm{TM}}$ transformers are an indication of the bcam losses between the two positions at these low proton energies. The ${ }^{24} \mathrm{Na}$ activation foil data were used in the engineering analysis results to be presented in this report. R. Cooper (Bechtel-Nevada) and G. Morgan (LANL) are analyzing the videotapes of the target phosphor for illumination distribution and the activation foils to determine the activity distribution. Both distributions will be used to finalize the estimates of the beam spot sizes on the targets to complete the engineering analyses of the data. The data from the videotapes of the target phosphor (analyzed by R. Cooper, et al.) agree with the initial visual estimates of the beam spot sizes for the two energies. The activation foil results (G. Morgan, et al.) have not been fully evaluated, but a preliminary evaluation of the results supports the beam spot size estimates used in the preliminary data analyses presented in this report.

Table 1

Results of Proton Dosimetry For AGS Experiment 945A

\begin{tabular}{||c|c|c|c|c||}
\hline Test & ${ }^{24} \mathrm{Na}$ Activity & ${ }^{22} \mathrm{Na}$ Activity & Bergoz ${ }^{\text {TM }}$ Coil & UXF3 GPM \\
\hline $\begin{array}{c}1.94 \mathrm{GeV}-1 \\
\text { (Foil 5A) }\end{array}$ & $3.22 \times 10^{15}$ & $3.28 \times 10^{15}$ & ----- & $3.94 \times 10^{15}$ \\
\hline $\begin{array}{c}1.94 \mathrm{GeV}-2 \\
(\text { Foil } 5 \mathrm{~B})\end{array}$ & $2.34 \times 10^{15}$ & $2.34 \times 10^{15}$ & $-\cdots$ & $2.95 \times 10^{15}$ \\
\hline $\begin{array}{c}1.94 \mathrm{GeV}-3 \\
(\text { Foil } 5 \mathrm{C})\end{array}$ & $4.95 \times 10^{14}$ & $5.00 \times 10^{14}$ & --- & $5.86 \times 10^{14}$ \\
\hline $\begin{array}{c}1.10 \mathrm{GeV}-1 \\
(\text { Foil } 6 \mathrm{~A})\end{array}$ & $3.75 \times 10^{14}$ & $3.73 \times 10^{14}$ & $3.83 \times 10^{14}$ & $1.52 \times 10^{15}$ \\
\hline $\begin{array}{c}1.10 \mathrm{GeV}-2 \\
(\text { Foil } 6 \mathrm{~B})\end{array}$ & $1.01 \times 10^{15}$ & $1.01 \times 10^{15}$ & $1.04 \times 10^{15}$ & $4.40 \times 10^{15}$ \\
\hline
\end{tabular}




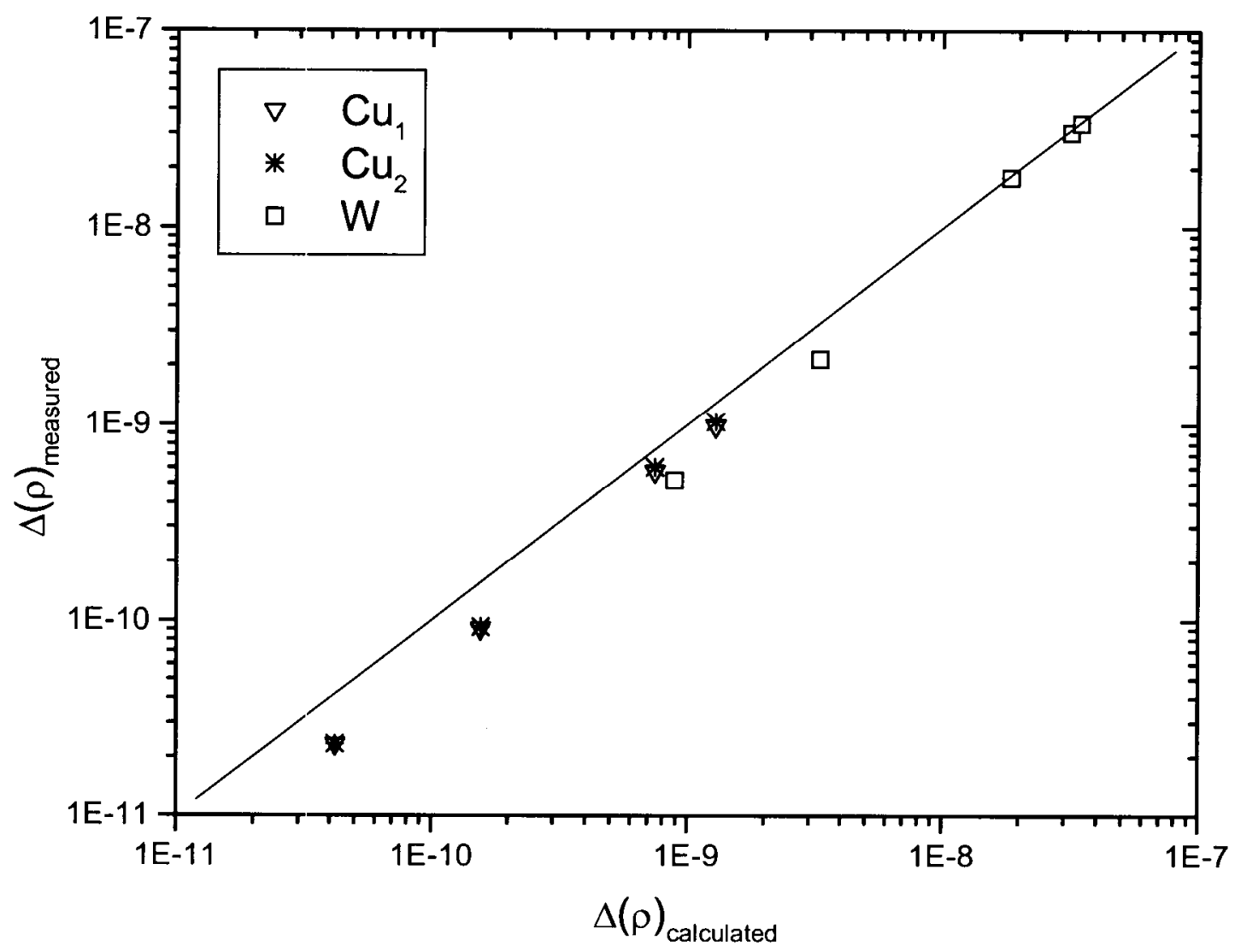

Figure 1. Comparison of the Measured Resistivity Increases of the Wire Samples to the Calculated Increases Due to 1.94 and 1.10 GeV Proton Irradiations (based upon measurements of ${ }^{24} \mathrm{Na}$ activity in aluminum activation foils; resistivity in units of $\left.\Omega-\mathrm{cm}\right)$.

\section{PRELIMINARY DATA ANALYSES AND OBSERVATIONS}

A preliminary engineering analysis of the experimental data was performed to convert the raw experimental measurements into measured electrical resistivity increases of the samples in order to compare to calculations of the predicted resistivity increases of the samples due to defect production in the wire samples. The data are presented in Figurc 1 as measured resistivity increases for both copper and tungsten vs. the resistivity increases that are calculated from parameters in the literature. The data are presented again in Figure 2 as ratios of the measured-to-calculated resistivity increases vs. the measured resistivity increases for each of the thirteen data points. The tungsten ratio is nearly unity for the $1.94-\mathrm{GeV}$ proton data and falls to 0.78 for the $1.10-\mathrm{GeV}$ data. The copper ratios are not as good, ranging from 0.62 for the $1.94-\mathrm{GeV}$-proton data to 0.57 for the 1.10 - 


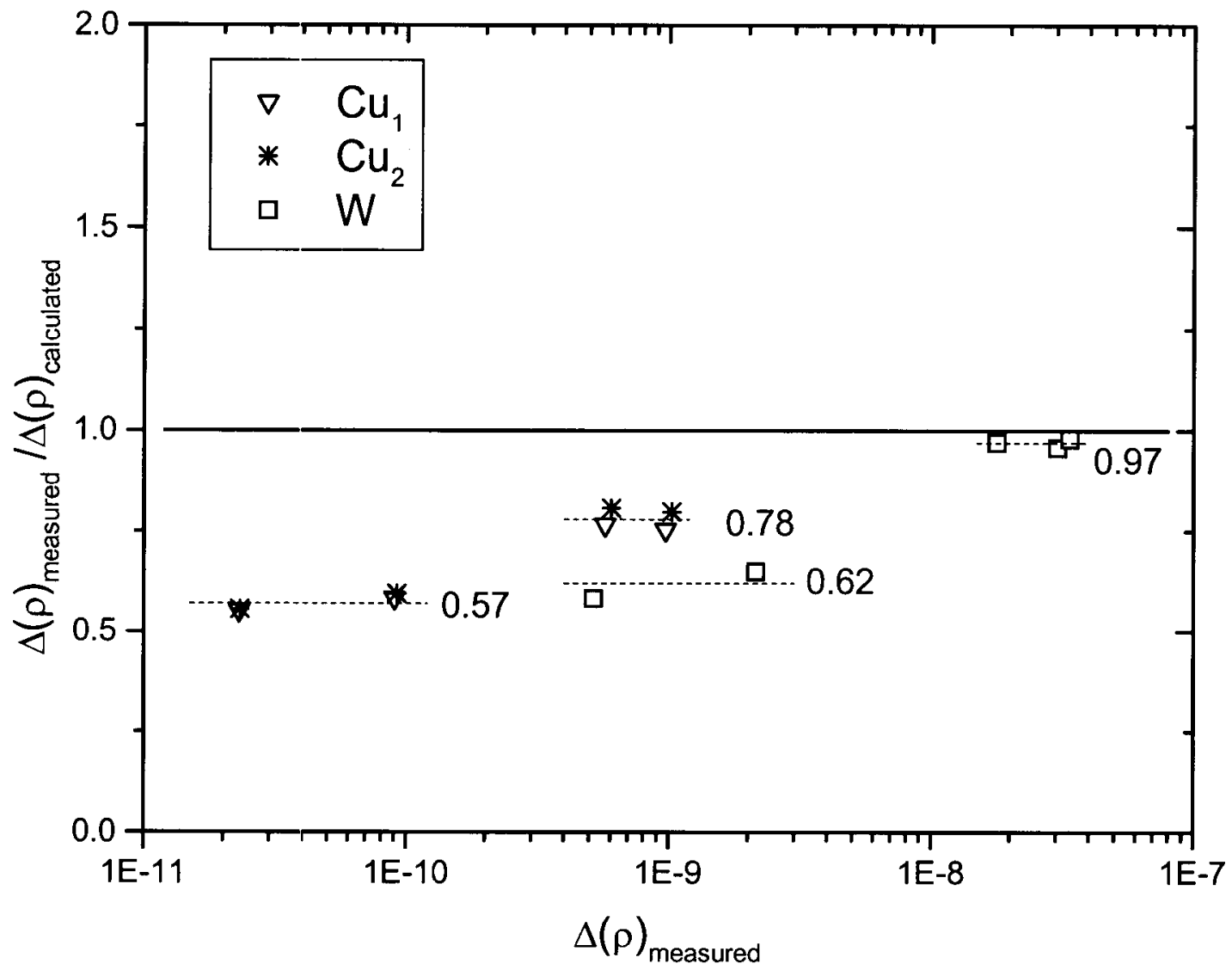

Figure 2. Comparison of the Ratios of the Measured Resistivity Increases of the Wire Samples to the Calculated Increases Due to 1.94 and $1.10 \mathrm{GeV}$ Proton Irradiations vs. the Measured Resistivity Increases (based upon measurements of ${ }^{24} \mathrm{Na}$ activity in aluminum activation foils; resistivity in units of $\Omega-\mathrm{cm})$.

$\mathrm{GeV}$ data. The predictions exceed the measured resistivity increases; the comparisons improve with increasing atomic number of the sample $(Z)$ and with increasing proton energy over the range of $Z$ and proton energy investigated. The experimental data and the parameters that went into the calculations of the predicted resistivity increases are tabulated in Table 2 which is an EXCEL spreadsheet. All the variables and their respective dimensions are defined in the spreadsheet. All formulae used in the engineering analyses are similarly defined in Table 2 . It is worth repeating that the beam spot areas used in the analyses, $A_{i}$, may yet be changed when the data from the analyses of the activation foils have been evaluated. The magnitude of the possible changes is not expected to be large and the effects as manifested through the measured-to-calculated resistivity ratios will be offsetting because both the measured and calculated resistivities are inversely proportional to $A_{i}$. 


\section{CONTENTS OF APPENDICES}

The bulk of the report consists of appendices to transmit and archive the results of the experiment. Appendix 1 contains the run sheets which were filled out manually during data acquisition in the beam line and later converted to electronic format after a quality control check. The resistance and proton fluence data indicated in bold face on these sheets are those used in Table 2. Appcndiccs 2, 3 and 4 contain the raw data for samples Copper-1, Copper-2 and Tungsten, as imported to EXCEL from text files. These have been converted from currents and voltages to resistance, and these are the resistances recorded on the run sheets in Appendix 1 and in Table 2. 


\section{Table 2}

EXCEL Spreadsheet for Preliminary Engineering Analysis of Experimental Data from AGS Experiment 945A:

Radiation Damage in Metals at Liquid Helium Temperature

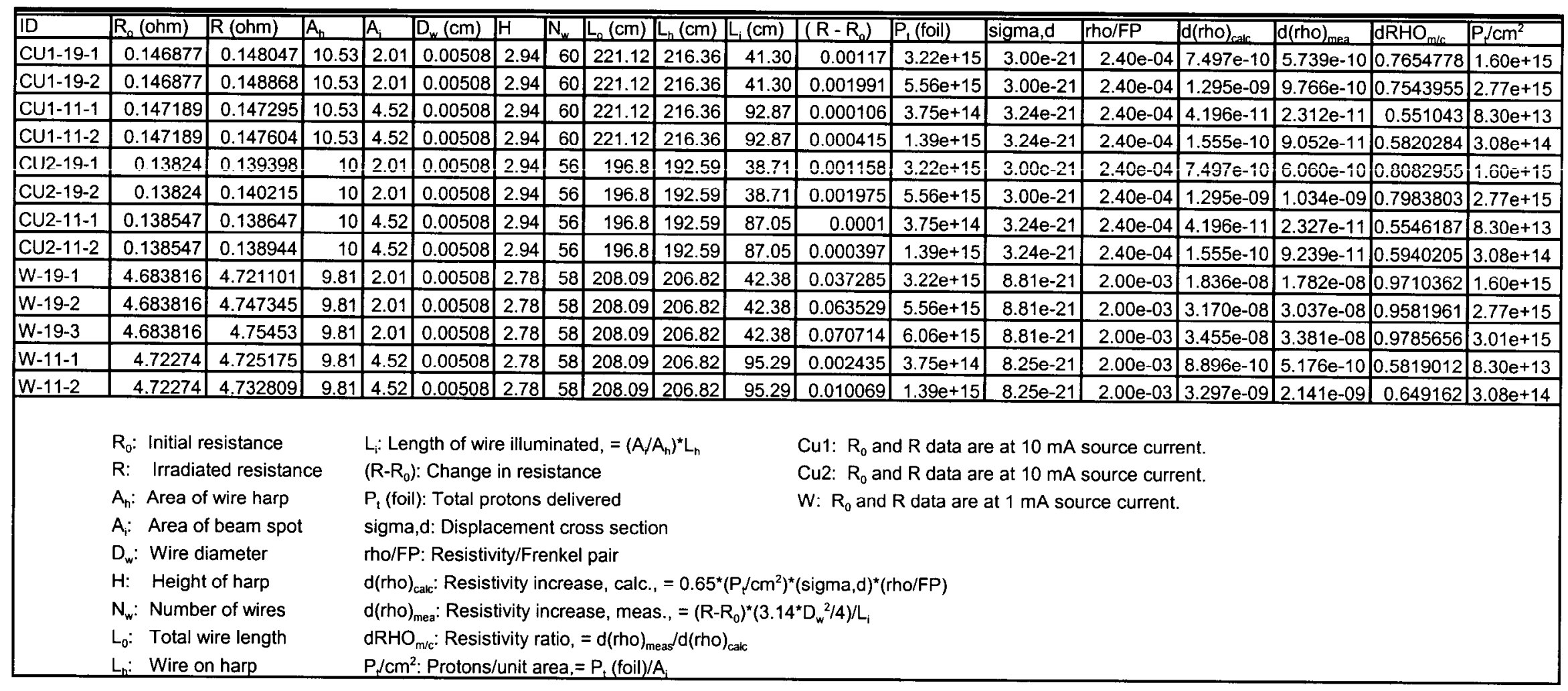


APPENDIX 1

EXPERIMENT RUN SHEETS

1.1 


\section{RADIATION DAMAGE BY ENERGETIC PROTONS}

Date, time:

$3 / 15 / 99,1630$ hours

Proton energy:

pre-1.94 GeV

Cumulative irradiation time: 0

Cumulative fluence:

0

Activation foil identification: none

\section{Room Temperature Resistance}

Cu \#1: $\quad$ Nominal resistance at $300 \mathrm{~K}$ is $18 \Omega$

Source current $(\mathrm{mA}): \quad 0.05 \quad 0.50$

Resistance $(\Omega)$ : $\quad 18.002148 \quad 18.000804$

Standard deviation $(\Omega): \quad 0.0001144 \quad 2.0488 \times 10^{-5}$

Temperature (K): $\quad 290.82 \quad 290.78$

Cu \#2: $\quad$ Nominal resistance at $300 \mathrm{~K}$ is $17 \Omega$

Source current (mA): $\quad 0.05 \quad 0.50$

Resistance $(\Omega)$ : $\quad 17.315271 \quad 17.314081$

Standard deviation $(\Omega): \quad 0.00010412 \quad 3.0088 \times 10^{-5}$

Temperature (K): $\quad 290.88 \quad 290.74$

W: $\quad$ Nominal resistance at $300 \mathrm{~K}$ is $60 \Omega$

Source current (mA): $\quad 0.05 \quad 0.50$

Resistance $(\Omega)$ : $\quad 59.986642 \quad 59.979019$

Standard deviation $(\Omega): \quad 0.00064685 \quad 0.00014088$

Temperature (K): $\quad 290.85 \quad 290.74$

Notes: These data were taken following installation of the apparatus in the U-line to evaluate the condition of the samples. 


\section{RADIATION DAMAGE BY ENERGETIC PROTONS}

Date, time:

3/16/99, 0600 hours

Proton energy: pre- $1.94 \mathrm{GeV}$

Cumulative irradiation time: 0

Cumulative fluence:

0

Activation foil identification: none

\section{Cryogenic Temperature Resistance}

Cu \#1:

Nominal resistance at $5 \mathrm{~K}$ is $0.150 \Omega$

Source current (mA):

5

0.146877

10

15

Resistance $(\Omega)$ :

$1.409 \times 10^{-6}$

0.146877

0.146876

Standard deviation $(\Omega)$ :

4.784

$5.5044 \times 10^{-7}$

$6.3207 \times 10^{-7}$

Temperature (K):

4.785

4.785

Cu \#2:

Nominal resistance at $5 \mathrm{~K}$ is $0.140 \Omega$

Source current (mA):

5

10

15

Resistance $(\Omega)$ :

0.138240

0.138240

0.138240

Standard deviation $(\Omega)$ :

$1.1783 \times 10^{-6}$

$5.1583 \times 10^{-7}$

$5.2773 \times 10^{-7}$

Temperature (K):

4.786

4.786

4.786

W:

Nominal resistance at $5 \mathrm{~K}$ is $4.700 \Omega$

Source current (mA):

0.5

1.0

2.0

Resistance $(\Omega)$ :

4.683817

$\mathbf{4 . 6 8 3 8 1 6}$

4.683718

Standard deviation $(\Omega)$ :

$1.4371 \times 10^{-5}$

$1.0598 \times 10^{-5}$

$1.1247 \times 10^{-5}$

Temperature $(\mathrm{K})$ :

4.787

4.787

4.787

Notes: LHe started at 0500 hours. Started data acquisition at 0600 hours. Data acquisition completed at 0630 hours. Resistances and proton fluences shown in bold face are those used in the EXCEL spreadsheet analyses. 


\section{RADIATION DAMAGE BY ENERGETIC PROTONS}

Date, time:

Proton energy:

$3 / 16 / 99,0725$ hours (first run)

Cumulative irradiation time: 90 minutes

Cumulative fluence: $\quad 3.22 \times 10^{15}\left({ }^{24} \mathrm{Na}\right.$ activation), $3.28 \times 10^{15}\left({ }^{22} \mathrm{Na}\right.$ activation), $3.94 \times 10^{15}(\mathrm{UXF} 3)$

Activation foil identification: $5 \mathrm{~A}$

\section{Cryogenic Temperature Resistance}

Cu \#1:

Nominal resistance at $5 \mathrm{~K}$ is $0.150 \Omega$

Source current (mA):

5

10

15

Resistance $(\Omega)$ :

0.148048

0.148047

0.148048

Standard deviation $(\Omega)$ :

$1.1633 \times 10^{-6}$

$5.2858 \times 10^{-7}$

$3.7631 \times 10^{-7}$

Temperature $(\mathrm{K})$ :

4.763

4.762

4.764

Cu \#2:

Nominal resistance at $5 \mathrm{~K}$ is $0.140 \Omega$

Source current (mA):

5

10

15

Resistance $(\Omega)$ :

0.139400

0.139398

0.139398

Standard deviation $(\Omega)$ :

$8.9797 \times 10^{-7}$

$3.5355 \times 10^{-7}$

$4.1268 \times 10^{-7}$

Temperature $(\mathrm{K})$ :

4.775

4.777

4.778

W:

Nominal resistance at $5 \mathrm{~K}$ is $4.700 \Omega$

Source current (mA):

Resistance ( $\Omega$ ):

Standard deviation $(\Omega)$ :

Temperature $(\mathrm{K})$ :
0.5

4.721110

$9.7792 \times 10^{-6}$

4.777
1.0

4.721101

$4.9594 \times 10^{-6}$

4.777
2.0

4.720945

$7.4193 \times 10^{-6}$

4.777

Notes: Bergoz coil is disabled. UXF3 (upstream of WD1) is reading $1.1 \times 10^{12} \mathrm{p} /$ spill every 1.4 seconds. The estimate is that Bergoz/UXF3 $\sim 0.8$. The irradiation started at 0725 hours, ended at 0855 hours. Started data acquisition at 1000 hours; completed at 1030 hours. Resistances and proton fluences shown in bold face are those used in the EXCEL spreadsheet analyses. 


\section{RADIATION DAMAGE BY ENERGETIC PROTONS}

Date, time:

Proton energy:

$3 / 16 / 99,1106$ hours (second run)

Cumulative irradiation time: 150 minutes

Cumulative fluence:

$5.56 \times 10^{15}\left({ }^{24} \mathrm{Na}\right.$ activation), $5.62 \times 10^{15}\left({ }^{22} \mathrm{Na}\right.$ activation $)$, $6.89 \times 10^{15}(\mathrm{UXF} 3)$

Activation foil identification: 5B

\section{Cryogenic Temperature Resistance}

\section{Cu \#1:}

Nominal resistance at $5 \mathrm{~K}$ is $0.150 \Omega$

Source current $(\mathrm{mA})$ :

Resistance $(\Omega)$ :

5

0.148869

10

15

Standard deviation $(\Omega)$ :

$9.1222 \times 10^{-7}$

0.148868

0.148868

Temperature (K):

4.791

$5.9903 \times 10^{-7} \quad 5.6651 \times 10^{-7}$

4.790

4.790

Cu \#2:

Nominal resistance at $5 \mathrm{~K}$ is $0.140 \Omega$

Source current (mA):

Resistance $(\Omega)$ :

5

10

15

Standard deviation $(\Omega)$ :

0.140216

0.140215

0.140215

Temperature (K):

$8.105 \times 10^{-7}$

$5.2625 \times 10^{-7}$

$5.0756 \times 10^{-7}$

4.790

4.790

4.790

$\mathbf{w}$ :

Nominal resistance at $5 \mathrm{~K}$ is $4.700 \Omega$

Source current $(\mathrm{mA})$ :

$$
0.5
$$

4.747354

1.0

2.0

Resistance $(\Omega)$ : $1.0294 \times 10^{-5}$

4.747345

4.747202

Standard deviation $(\Omega)$ :

4.790

$6.3057 \times 10^{-6}$

$7.5646 \times 10^{-6}$

Temperature $(\mathrm{K})$ :

4.789

4.790

Notes: Irradiation started at 1106 hours. Irradiation ended at 1206 hours. Resistances and proton fluences shown in bold face are those used in the EXCEL spreadsheet analyses. 


\section{RADIATION DAMAGE BY ENERGETIC PROTONS}

Date, time:

Proton energy:

$3 / 16 / 99,1333$ hours (third run)

Cumulative irradiation time:

$1.94 \mathrm{GeV}$

Cumulative fluence:

163 minutes

Activation foil identification: $5 \mathrm{C}$

$6.06 \times 10^{15}\left({ }^{24} \mathrm{Na}\right.$ activation), $6.12 \times 10^{15}\left({ }^{22} \mathrm{Na}\right.$ activation), $7.48 \times 10^{15}$ (UXF3)

\section{Cryogenic Temperature Resistance}

Cu \#1:

Norninal resistance at $5 \mathrm{~K}$ is $0.150 \Omega$

Source current (mA):

Resistance $(\Omega)$ :

5

0.148760

10

15

Standard deviation $(\Omega)$ :

$1.7283 \times 10^{-6}$

0.148755

$\operatorname{xxxxxx}$

7.141

$9.9034 \times 10^{-7}$

$\operatorname{xxxxxx}$

Temperature $(\mathrm{K})$ :

7.206

$\operatorname{xxxxxx}$

Cu \#2:

Norninal resistance at $5 \mathrm{~K}$ is $0.140 \Omega$

Source current (mA):

Resistance $(\Omega)$ :

5

0.140026

10

0.139988

15

1

Standard deviation $(\Omega)$ :

$2.0743 \times 10^{-6}$

$1.2202 \times 10^{-6}$

$\operatorname{xxxxxx}$

0.140024

Temperature $(\mathrm{K})$ :

7.28

7.67

$\operatorname{xxxxxx}$

$\operatorname{xxxxxx}$

$4.4325 \times 10^{-6}$

7.33

W:

Norninal resistance at $5 \mathrm{~K}$ is $4.700 \Omega$

Source current (mA):

0.5

1.0

2.0

Resistance $(\Omega)$ :

4.754621

4.754530

4.754415

Standard deviation $(\Omega)$ :

$1.5157 \times 10^{-5}$

$8.856 \times 10^{-6}$

7.58

7.43

$1.4665 \times 10^{-5}$

Temperature (K):

7.48

Notes: Irradiation started at 1333 hours. Irradiation was terminated at 1345 hours due to a sudden reduction in LHe flow from $4 \mathrm{lphr}$ to $2 \mathrm{lphr}$. The Bergoz readings are: NTRIG $=489$, $\mathrm{C} 2 \mathrm{~F}=-169.482, \mathrm{C} 3 \mathrm{~F}=-180.117, \mathrm{C} 2 \mathrm{~B}=3.333, \mathrm{C} 3 \mathrm{~B}=2.191$. Coil $2=4.54 \times 10^{14}$ protons, coil 3 $=4.49 \times 10^{14}$ protons, for an average of $4.52 \times 10^{14}$ protons. UXF3 gives $5.86 \times 10^{14}$ protons for this run. There were two consecutive runs at $0.5 \mathrm{~mA}$ on the tungsten sample which gave false readings. The reading was repeated until a correct reading was made. The two false readings are still in the data base, uncorrected. Resistances and proton fluences shown in bold face are those used in the EXCEL spreadsheet analyses. 


\section{RADIATION DAMAGE BY ENERGETIC PROTONS}

Date, time:

Proton energy:

Cumulative irradiation time:

Cumulative fluence:

Activation foil identification: $5 \mathrm{~A}, 5 \mathrm{~B}, 5 \mathrm{C}$
3/16/99, 1600 hours

post- $1.94 \mathrm{GeV}$

\section{Room Temperature Resistance}

Cu \#1: $\quad$ Norninal resistance at $300 \mathrm{~K}$ is $18 \Omega$

$\begin{array}{lll}\text { Source current }(\mathrm{mA}): & 0.05 & 0.50 \\ \text { Resistance }(\Omega): & 17.67165 & 17.66832 \\ \text { Standard deviation }(\Omega): & 0.0023 & 0.00169 \\ \text { Temperature }(\mathrm{K}): & 290.23 & 290.29\end{array}$

Cu \#2:

Norninal resistance at $300 \mathrm{~K}$ is $17 \Omega$

$\begin{array}{lll}\text { Source current }(\mathrm{mA}): & 0.05 & 0.50 \\ \text { Resistance }(\Omega): & 17.04075 & 17.076771 \\ \text { Standard deviation }(\Omega): & 0.00479 & 0.00757 \\ \text { Temperature }(\mathrm{K}): & 290.69 & 291.34\end{array}$

W: $\quad$ Norninal resistance at $300 \mathrm{~K}$ is $60 \Omega$

$\begin{array}{lll}\text { Source current }(\mathrm{mA}): & 0.05 & 0.50 \\ \text { Resistance }(\Omega): & 59.617638 & 59.805468 \\ \text { Standard deviation }(\Omega): & 0.032612 & 0.03737 \\ \text { Temperature }(\mathrm{K}): & 292.23 & 293.04\end{array}$

Notes: These temperatures may not be at steady state; the samples may still be colder, still in a warm-up transient. This is supported by the standard deviations. Only ten readings were taken per batch to minimize the effect of the room temperature variations on the standard deviation. Resistances and proton fluences shown in bold face are those used in the EXCEL spreadsheet analyses. 


\section{RADIATION DAMAGE BY ENERGETIC PROTONS}

Date, time:

3/17/99, 0830 hours

Proton energy:

pre-1.10 GeV

Cumulative irradiation time: 0

Cumulative fluence:

0

Activation foil identification: none

\section{Room Temperature Resistance}

Cu \#1:

Nominal resistance at $300 \mathrm{~K}$ is $18 \Omega$

Source current (mA):

0.05

0.50

Resistance $(\Omega)$ :

17.973918

17.972512

Standard deviation $(\Omega)$ :

0.00011876

$3.0824 \times 10^{-5}$

Temperature (K):

290

290

Cu \#2:

Norninal resistance at $300 \mathrm{~K}$ is $17 \Omega$

Source current (mA):

0.05

0.50

Resistance ( $\Omega$ ):

17.288274

17.287024

Standard deviation $(\Omega)$ :

$9.9049 \times 10^{-5}$

$3.9359 \times 10^{-5}$

Temperature (K):

291

290

W:

Norninal resistance at $300 \mathrm{~K}$ is $60 \Omega$

Source current (mA):

0.05

0.50

Resistance $(\Omega)$ :

59.931729

59.923370

Standard deviation $(\Omega)$ :

$1.9778 \times 10^{-4}$

290

$2.2418 \times 10^{-4}$

Temperature (K):

291

Notes: This is the warm data after $1.94-\mathrm{GeV}$ and prior to $1.10-\mathrm{GeV}$. The apparatus had overnight to equilibrate to room temperature. 


\section{RADIATION DAMAGE BY ENERGETIC PROTONS}

Date, time:

$3 / 17 / 99,1000$ hours

Proton energy:

pre-1.10 GeV

Cumulative irradiation time: 0

Cumulative fluence:

0

Activation foil identification: none

\section{Cryogenic Temperature Resistance}

Cu \#1: $\quad$ Nominal resistance at $5 \mathrm{~K}$ is $0.150 \Omega$

$\begin{array}{lllll}\text { Source current }(\mathrm{m} \Lambda): & 5 & 10 & 15 & 10 \text { (at } 1025 \text { hours) } \\ \text { Resistance }(\Omega): & 0.147189 & \mathbf{0 . 1 4 7 1 8 9} & 0.147189 & 0.147190 \\ \text { Standard deviation }(\Omega): & 9.086 \times 10^{-7} & 4.8123 \times 10^{-7} & 4.9868 \times 10^{-7} & 4.7249 \times 10^{-7} \\ \text { Temperature }(\mathrm{K}): & 4.728 & 4.728 & 4.728 & 4.727\end{array}$

Cu \#2: $\quad$ Nominal resistance at $5 \mathrm{~K}$ is $0.140 \Omega$

$\begin{array}{llll}\text { Source current }(\mathrm{mA}): & 5 & 10 & 15 \\ \text { Resistance }(\Omega): & 0.138547 & \mathbf{0 . 1 3 8 5 4 7} & 0.138547 \\ \text { Standard deviation }(\Omega): & 1.1993 \times 10^{-6} & 5.7923 \times 10^{-7} & 3.844 \times 10^{-7} \\ \text { Temperature }(\mathrm{K}): & 4.728 & 4.728 & 4.727\end{array}$

W: $\quad$ Norninal resistance at $5 \mathrm{~K}$ is $4.700 \Omega$

$\begin{array}{llll}\text { Source current }(\mathrm{mA}): & 0.5 & 1.0 & 2.0 \\ \text { Resistance }(\Omega): & 4.722742 & \mathbf{4 . 7 2 2 7 4 0} & 4.722625 \\ \text { Standard deviation }(\Omega): & 1.9556 \times 10^{-5} & 1.5127 \times 10^{-5} & 1.6891 \times 10^{-5} \\ \text { Temperature }(\mathrm{K}): & 4.727 & 4.727 & 4.727\end{array}$

Notes: Entry into beam line at 0800 hours. Started LHe flow at 0910 hours. The dewar has 54 liters. The vacuum is $5 \times 10^{-8}$ Torr. The Cernox reads $4.82 \mathrm{~K}$ at 0940 hours, $4.72 \mathrm{~K}$ at 0950 hours, $4.727 \mathrm{~K}$ at 0954 hours. The data acquisition was started at 1000 hours and finished at 1030 hours. The last measurement was made to see if there was a measurable temperature transient in the wires over the 30 minute time interval. None was measured. Resistances and proton fluences shown in bold face are those used in the EXCEL spreadsheet analyses. 


\section{RADIATION DAMAGE BY ENERGETIC PROTONS}

Date, time:

Proton energy:

3/17/99, 1140 hours (first run)

Cumulative fluence:

$1.10 \mathrm{GeV}$

Cumulative irradiation time: 60 minutes

Activation foil identification: $6 \mathrm{~A}$

$3.75 \times 10^{14}\left({ }^{24} \mathrm{Na}\right.$ activation), $3.73 \times 10^{14}\left({ }^{22} \mathrm{Na}\right.$ activation), $1.52 \times 10^{15}$ (UXF3), 3.83 $\times 10^{14}$ (Bergoz)

Cryogenic Temperature Resistance

Cu \#1: $\quad$ Norninal resistance at $5 \mathrm{~K}$ is $0.150 \Omega$

$\begin{array}{llll}\text { Source current }(\mathrm{mA}): & 5 & 10 & 15 \\ \text { Resistance }(\Omega): & 0.147295 & \mathbf{0 . 1 4 7 2 9 5} & 0.147294 \\ \text { Standard deviation }(\Omega): & 1.2954 \times 10^{-6} & 4.5629 \times 10^{-7} & 5.5046 \times 10^{-7} \\ \text { Temperature }(\mathrm{K}): & 4.716 & 4.718 & 4.719\end{array}$

Cu \#2: $\quad$ Norninal resistance at $5 \mathrm{~K}$ is $0.140 \Omega$

$\begin{array}{llll}\text { Source current }(\mathrm{mA}): & 5 & 10 & 15 \\ \text { Resistancc }(\Omega): & 0.138647 & \mathbf{0 . 1 3 8 6 4 7} & 0.138646 \\ \text { Standard deviation }(\Omega): & 6.7566 \times 10^{-7} & 4.3243 \times 10^{-7} & 5.3004 \times 10^{-7} \\ \text { Temperature }(\mathrm{K}): & 4.719 & 4.719 & 4.720\end{array}$

W: $\quad$ Norainal resistance at $5 \mathrm{~K}$ is $4.700 \Omega$

$\begin{array}{lllll}\text { Source current }(\mathrm{mA}): & 0.5 & 1.0 & 1.0 & 2.0 \\ \text { Resistance }(\Omega): & 4.725175 & 4.725175 & \mathbf{4 . 7 2 5 1 7 5} & 4.725060 \\ \text { Standard deviation }(\Omega): & 6.9794 \times 10^{-6} & 5.9327 \times 10^{-6} & 5.7838 \times 10^{-6} & 1.277 \times 10^{-5} \\ \text { Temperature }(\mathrm{K}): & 4.720 & 4.720 & 4.720 & 4.721\end{array}$

Notes: The vacuum is holding at $2 \times 10^{-8}$ Torr. Beam started at 1140 hours, ended at 1240 hours. Start data acquisition at 1305 hours, finish at 1330 hours. The Bergoz readings are: NTRIG = $1281, \mathrm{C} 2 \mathrm{~F}=-53.574, \mathrm{C} 3 \mathrm{~F}=-55.999, \mathrm{C} 2 \mathrm{~B}=3.438, \mathrm{C} 3 \mathrm{~B}=1.949$. Coil $2=3.926 \times 10^{14}$ protons, coil $3=3.739 \times 10^{14}$ protons, for an average of $3.83 \times 10^{14}$ protons. Resistances and proton fluences shown in bold face are those used in the EXCEL spreadsheet analyses. 


\section{RADIATION DAMAGE BY ENERGETIC PROTONS}

Date, time:

Proton energy:

$3 / 17 / 99,1345$ hours (second run)

Cumulative irradiation time: 135 minutes

Cumulative fluence:

$1.39 \times 10^{15}\left({ }^{24} \mathrm{Na}\right.$ activation), $1.39 \times 10^{15}\left({ }^{22} \mathrm{Na}\right.$ activation $)$, $5.92 \times 10^{15}$ (UXF3), $1.42 \times 10^{15}$ (Bergoz)

Activation foil identification: $6 \mathrm{~B}$

\section{Cryogenic Temperature Resistance}

Cu \#1: $\quad$ Nominal resistance at $5 \mathrm{~K}$ is $0.150 \Omega$

$\begin{array}{llll}\text { Source current }(\mathrm{mA}): & 5 & 10 & 15 \\ \text { Resistance }(\Omega): & 0.147605 & \mathbf{0 . 1 4 7 6 0 4} & 0.147604 \\ \text { Standard deviation }(\Omega): & 5.2927 \times 10^{-7} & 5.0473 \times 10^{-7} & 4.0219 \times 10^{-7} \\ \text { Temperature }(\mathrm{K}): & 4.726 & 4.727 & 4.726\end{array}$

Cu \#2: $\quad$ Norninal resistance at $5 \mathrm{~K}$ is $0.140 \Omega$

$\begin{array}{llll}\text { Source current }(\mathrm{mA}): & 5 & 10 & 15\end{array}$

$\begin{array}{llll}\text { Resistance }(\Omega): & 0.138946 & \mathbf{0 . 1 3 8 9 4 4} & 0.138945\end{array}$

$\begin{array}{llll}\text { Standard deviation }(\Omega): & 8.8777 \times 10^{-7} & 3.6685 \times 10^{-7} & 3.3223 \times 10^{-7}\end{array}$

Temperature (K): $\quad 4.726 \quad 4.726 \quad 4.727$

W: $\quad$ Norninal resistance at $5 \mathrm{~K}$ is $4.700 \Omega$

$\begin{array}{llll}\text { Source current }(\mathrm{mA}): & 0.5 & 1.0 & 2.0 \\ \text { Resistance }(\Omega): & 4.732822 & \mathbf{4 . 7 3 2 8 0 9} & 4.732626 \\ \text { Standard deviation }(\Omega): & 8.4794 \times 10^{-6} & 5.3722 \times 10^{-6} & 8.1206 \times 10^{-6} \\ \text { Temperature }(\mathrm{K}): & 4.727 & 4.726 & 4.727\end{array}$

Notes: Vacuum is holding at $9.5 \times 10^{-9}$ Torr at the start of data acquisition. The beam was started at 1345 hours, shut off at 1500 hours and data acquisition started at 1530 hours. The Bergoz readings are: $\mathrm{NTRIG}=2987, \mathrm{C} 2 \mathrm{~F}=-62.97, \mathrm{C} 3 \mathrm{~F}=-64.976, \mathrm{C} 2 \mathrm{~B}=3.335, \mathrm{C} 3 \mathrm{~B}=2.635$. Coil $2=$ $1.065 \times 10^{15}$ protons, coil $3=1.017 \times 10^{15}$ protons, for an average of $1.041 \times 10^{15}$ protons. There were 54 liters of LHe at 0900 hours, 21 liters left at 1600 hours, 33 liters in 7 hours $\sim 4.7 \mathrm{lphr}$. At this consumption rate, the LHe would have lasted until 2030 hours. Resistances and proton fluences shown in bold face are those used in the EXCEL spreadsheet analyses. 


\section{RADIATION DAMAGE BY ENERGETIC PROTONS}

Date, time:

3/19/99, 1345 hours

Proton energy: post- $1.10 \mathrm{GeV}$

Cumulative irradiation time: 135 minutes

Cumulative fluence:

$1.39 \times 10^{15}\left({ }^{24} \mathrm{Na}\right.$ activation), $1.39 \times 10^{15}\left({ }^{22} \mathrm{Na}\right.$ activation), $5.92 \times 10^{15}$ (UXF3), $1.42 \times 10^{15}$ (Bergoz)

Activation foil identification: $6 \mathrm{~A}, 6 \mathrm{~B}$

\section{Room Temperature Resistance}

Cu \#1:

Norninal resistance at $300 \mathrm{~K}$ is $18 \Omega$

Source current (mA):

Resistance $(\Omega)$ :

Standard deviation $(\Omega)$ :

Temperature $(\mathrm{K})$ :

$\begin{array}{ll}0.05 & 0.50 \\ 17.9428937 & 17.9408250 \\ 6.00068 \times 10^{-4} & 9.12000 \times 10^{-5} \\ 290.1 & 290.0\end{array}$

Norninal resistance at $300 \mathrm{~K}$ is $17 \Omega$

$\mathrm{Cu} \# 2$ :

$\begin{array}{ll}0.05 & 0.50 \\ 17.2573124 & 17.2555178 \\ 1.19776 \times 10^{-4} & 8.29000 \times 10^{-5} \\ 290.0 & 290.0\end{array}$

W:

Nominal resistance at $300 \mathrm{~K}$ is $60 \Omega$

Source current (mA):

Resistance $(\Omega)$ :

0.05

0.50

Standard deviation $(\Omega)$ :

59.825758

59.815396

$2.96171 \times 10^{-4}$

$2.12581 \times 10^{-4}$

Temperature $(\mathrm{K})$ :
290.0

Notes: The apparatus has had 46 hours to warm up to room temperature and stabilize. Resistances and proton fluences shown in bold face are those used in the EXCEL spreadsheet analyses. 


\section{RADLATION DAMAGE BY ENERGETIC PROTONS}

Date, time:

Proton energy:

Cumulative irradiation time: 135 minutes

Cumulative fluence:

$1.39 \times 10^{15}\left({ }^{24} \mathrm{Na}\right.$ activation), $1.39 \times 10^{15}\left({ }^{22} \mathrm{Na}\right.$ activation), $5.92 \times 10^{15}$ (UXF3), $1.42 \times 10^{15}$ (Bergoz)
$6 / 22 / 99,1535$ hours

post-1.10 GeV

Activation foil identification: $6 \mathrm{~A}, 6 \mathrm{~B}$

\section{Activation foil identification: $6 \mathrm{~A}$, $6 \mathrm{~B}$}

\section{Cryogenic Temperature Resistance}

Cu \#1:

Norninal resistance at $5 \mathrm{~K}$ is $0.150 \Omega$

$\begin{array}{llll}\text { Source current }(\mathrm{mA}): & 5 & 10 & 15 \\ \text { Resistance }(\Omega): & 0.146908 & \mathbf{0 . 1 4 6 9 0 8} & 0.146906 \\ \text { Standard deviation }(\Omega): & 1.1006 \times 10^{-6} & 6.0296 \times 10^{-7} & 5.9648 \times 10^{-7} \\ \text { Temperature }(\mathrm{K}): & 4.827 & 4.828 & 4.828\end{array}$

Cu \#2:

Norninal resistance at $5 \mathrm{~K}$ is $0.140 \Omega$

Source current (mA):

Resistance $(\Omega)$ :

Standard deviation $(\Omega)$ :

Temperature (K):

$\begin{array}{lll}5 & 10 & 15 \\ 0.138270 & \mathbf{0 . 1 3 8 2 7 0} & 0.138268 \\ 1.4595 \times 10^{-6} & 7.5048 \times 10^{-7} & 5.9523 \times 10^{-7} \\ 4.829 & 4.829 & 4.829\end{array}$

Nominal resistance at $5 \mathrm{~K}$ is $4.700 \Omega$

Source current (mA): $\quad 0.5 \quad 1.0 \quad 2.0$

Resistance $(\Omega)$

4.726346

4.726338

4.726189

Standard deviation $(\Omega)$ :

$1.9156 \times 10^{-5}$

$1.1016 \times 10^{-5}$

4.829

$1.5547 \times 10^{-5}$

4.829

Notes: Vacuum is holding at $6.3 \times 10^{-9}$ Torr at the start of data acquisition. Samples were at room temperature under vacuum for three months since the end of the experiments in March 1999. Resistances and proton fluences shown in bold face are those used in the EXCEL spreadsheet analyses. 
APPENDIX 2

EXCEL IATA FILE OF RESISTANCE MEASUREMENTS

OF COPPER SAMPLE \#1 
Copper1.xls

\begin{tabular}{|c|c|c|c|c|c|c|c|c|}
\hline Current (A) & Voltage (V) & Res.(ohms) & Std. Dev. on $\Omega$ & Power (W) & Date/time & Cernox (ohms) & \multicolumn{2}{|c|}{ Cernox $(K) 4$ th order poly. } \\
\hline \multicolumn{9}{|c|}{ Pre-irradiation resistivity data at room temperature prior to testing at $1.94 \mathrm{GeV}$} \\
\hline & & & & & & & & \\
\hline $5.000259 \mathrm{E}-05$ & $-9.004734 \mathrm{E}-04$ & & & & $3 / 16 / 994: 30: 13$ & & & \\
\hline$-5.000480 \mathrm{E}-05$ & $8.999057 \mathrm{E}-04$ & & & & $3 / 16 / 994: 30: 19$ & & & \\
\hline $5.000259 \mathrm{E}-05$ & $-9.004365 E-04$ & & & & $3 / 16 / 994: 30: 22$ & & & \\
\hline $5.000264 \mathrm{E}-05$ & $-9.004270 \mathrm{E}-04$ & & & & $3 / 16 / 994: 30: 25$ & & & \\
\hline$-5.000479 \mathrm{E}-05$ & $8.999270 \mathrm{E}-04$ & & & & $3 / 16 / 994: 30: 27$ & & & \\
\hline $5.000263 \mathrm{E}-05$ & $-9.004022 E-04$ & & & & $3 / 16 / 994: 30: 36$ & & & \\
\hline$-5.000476 \mathrm{E}-05$ & $8.999429 \mathrm{E}-04$ & & & & $3 / 16 / 994: 30: 39$ & & & \\
\hline$-5.000478 \mathrm{E}-05$ & $8.999686 \mathrm{E}-04$ & & & & $3 / 16 / 994: 30: 41$ & & & \\
\hline $5.000263 \mathrm{E}-05$ & $-9.004001 \mathrm{E}-04$ & & & & $3 / 16 / 994: 30: 44$ & & & \\
\hline $5.000261 \mathrm{E}-05$ & $-9.003764 E-04$ & & & & $3 / 16 / 994: 30: 47$ & & & \\
\hline$-5.000479 E-05$ & $8.999804 \mathrm{E}-04$ & & & & $3 / 16 / 994: 30: 50$ & & & \\
\hline$-5.000479 \mathrm{E}-05$ & 8.999896E-04 & & & & $3 / 16 / 994: 30: 53$ & & & \\
\hline $5.000261 \mathrm{E}-05$ & $-9.003430 \mathrm{E}-04$ & & & & $3 / 16 / 994: 31: 10$ & & & \\
\hline$-5.000480 \mathrm{E}-05$ & $9.000028 \mathrm{E}-04$ & & & & $3 / 16 / 994: 31: 13$ & & & \\
\hline$-5.000475 \mathrm{E}-05$ & $9.000077 \mathrm{E}-04$ & & & & $3 / 16 / 994: 31: 15$ & & & \\
\hline $5.000264 \mathrm{E}-05$ & $-9.003421 E-04$ & & & & $3 / 16 / 994: 31: 18$ & & & \\
\hline $5.000267 \mathrm{E}-05$ & $-9.003451 E-04$ & & & & $3 / 16 / 99$ 4:31:21 & & & \\
\hline$-5.000484 \mathrm{E}-05$ & $9.000224 \mathrm{E}-04$ & & & & $3 / 16 / 99$ 4:31:24 & & & \\
\hline$-5.000484 \mathrm{E}-05$ & $9.000029 \mathrm{E}-04$ & & & & $3 / 16 / 994: 31: 27$ & & & \\
\hline $5.000263 \mathrm{E}-05$ & $-9.003367 E-04$ & & & & $3 / 16 / 994: 31: 30$ & & & \\
\hline $5.000265 \mathrm{E}-05$ & $-9.003339 E-04$ & & & & $3 / 16 / 994: 31: 32$ & & & \\
\hline$-5.000489 E-05$ & $9.000130 \mathrm{E}-04$ & & & & $3 / 16 / 994: 31: 35$ & & & \\
\hline$-5.000482 \mathrm{E}-05$ & $9.000247 \mathrm{E}-04$ & & & & $3 / 16 / 994: 31: 38$ & & & \\
\hline $5.000264 \mathrm{E}-05$ & $-9.003148 E-04$ & & & & $3 / 16 / 99$ 4:31:41 & & & \\
\hline $5.000263 \mathrm{E}-05$ & $-9.003165 \mathrm{E}-04$ & & & & 3/16/99 4:31:44 & & & \\
\hline$-5.000487 E-05$ & $9.000174 \mathrm{E}-04$ & & & & $3 / 16 / 99$ 4:31:47 & & & \\
\hline
\end{tabular}


Copper1.xis

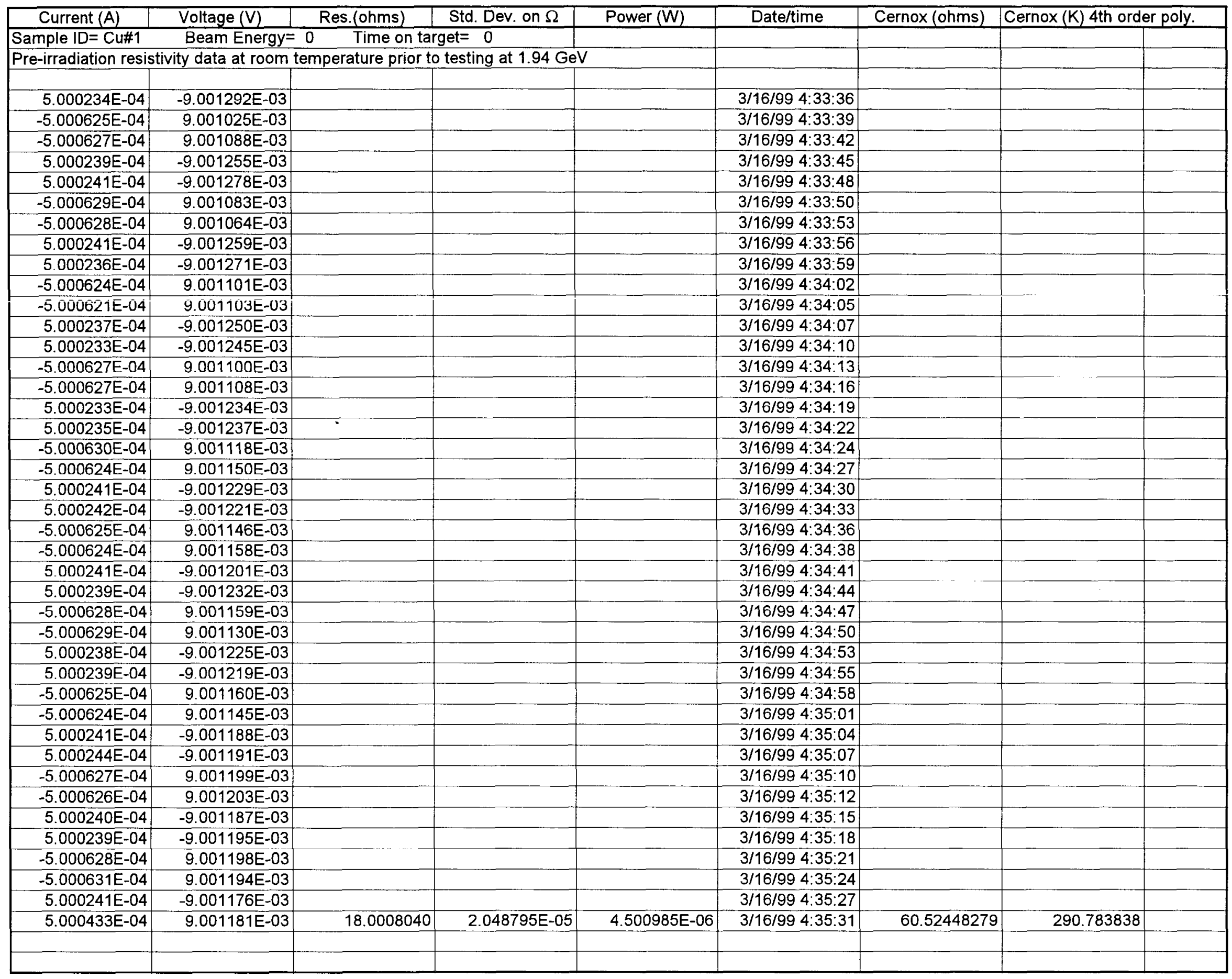


Copper1.xls

\begin{tabular}{|c|c|c|c|c|c|c|c|c|}
\hline Current $(\mathrm{A})$ & Voltage (V) & Res.(ohms) & Std. Dev. on $\Omega$ & Power (W) & Date/time & Cernox (ohms) & Cernox (K) 4th ord & er poly. \\
\hline Sample ID= Cu\#1 & Beam Energy= & $0 \quad$ Time on ta & get $=0$ & & & & & \\
\hline Pre-irradiation resis & vity data at liquid & ielium temperature & prior to testing at & $34 \mathrm{GeV}$ & & & & \\
\hline & & & & & & & & \\
\hline 5.000198E-03 & $-7.364354 \mathrm{E}-04$ & & & & $3 / 16 / 996: 10: 08$ & & & \\
\hline$-5.000710 \mathrm{E}-03$ & $7.324545 \mathrm{E}-04$ & & & & $3 / 16 / 996: 10: 11$ & & & \\
\hline$-5.000709 \mathrm{E}-03$ & $7.324722 \mathrm{E}-04$ & & & & $3 / 16 / 996: 10: 14$ & & & \\
\hline $5.000200 \mathrm{E}-03$ & $-7.364356 \mathrm{E}-04$ & & & & 3/16/99 6:10:17 & & & \\
\hline 5.000194E-03 & $-7.364339 \mathrm{E}-04$ & & & & $3 / 16 / 996: 10: 19$ & & & \\
\hline$-5.000712 \mathrm{E}-03$ & $7.325089 \mathrm{E}-04$ & & & & $3 / 16 / 996: 10: 22$ & & & \\
\hline$-5.000711 \mathrm{E}-03$ & $7.324807 \mathrm{E}-04$ & & & & $3 / 16 / 996: 10: 25$ & & & \\
\hline 5.000199E-03 & $-7.363978 \mathrm{E}-04$ & & & & $3 / 16 / 996: 10: 28$ & & & \\
\hline $5.000200 \mathrm{E}-03$ & $-7.364033 E-04$ & & & & $3 / 16 / 996: 10: 31$ & & & \\
\hline$-5.000708 \mathrm{E}-03$ & $7.324935 \mathrm{E}-04$ & & & & $3 / 16 / 996: 10: 34$ & & & \\
\hline$-5.000704 E-03$ & $7.3249 / 8$ 七 -04 & & & & $3 / 16 / 996: 10: 36$ & & & \\
\hline $5.000205 \mathrm{E}-03$ & $-7.363924 E-04$ & & & & $3 / 16 / 996: 10: 39$ & & & \\
\hline 5.000204E-03 & $-7.363935 E-04$ & & & & $3 / 16 / 996: 10: 42$ & & & \\
\hline$-5.000711 \mathrm{E}-03$ & $7.325069 \mathrm{E}-04$ & & & & $3 / 16 / 996: 10: 45$ & & & \\
\hline$-5.000710 \mathrm{E}-03$ & $7.325169 \mathrm{E}-04$ & & & & $3 / 16 / 996: 10: 48$ & & & \\
\hline $5.000202 \mathrm{E}-03$ & $-7.363815 E-04$ & & & & $3 / 16 / 996: 10: 51$ & & & \\
\hline $5.000202 E-03$ & $-7.363938 E-04$ & & & & $3 / 16 / 996: 10: 53$ & & & \\
\hline$-5.000715 \mathrm{E}-03$ & $7.325289 \mathrm{E}-04$ & & & & $3 / 16 / 996: 10: 56$ & & & \\
\hline$-5.000709 \mathrm{E}-03$ & $7.325128 \mathrm{E}-04$ & & & & $3 / 16 / 996: 10: 59$ & & & \\
\hline $5.000202 E-03$ & -7.363790 E-04 & & & & $3 / 16 / 996: 11: 02$ & & & \\
\hline $5.000206 \mathrm{E}-03$ & $-7.363730 \mathrm{E}-04$ & & & & $3 / 16 / 996: 11: 05$ & & & \\
\hline$-5.000720 \mathrm{E}-03$ & $7.325240 \mathrm{E}-04$ & & & & $3 / 16 / 996: 11: 08$ & & & \\
\hline$-5.000717 E-03$ & $7.325200 \mathrm{E}-04$ & & & & $3 / 16 / 996: 11: 10$ & & & \\
\hline $5.000198 \mathrm{E}-03$ & $-7.363952 E-04$ & & & & $3 / 16 / 996: 11: 13$ & & & \\
\hline 5.000196E-03 & $-7.363936 \mathrm{E}-04$ & & & & $3 / 16 / 996: 11: 16$ & & & \\
\hline$-5.000714 \mathrm{E}-03$ & $7.325092 \mathrm{E}-04$ & & & & $3 / 16 / 996: 11: 19$ & & & \\
\hline$-5.000712 \mathrm{E}-03$ & $7.325105 E-04$ & & & & $3 / 16 / 996: 11: 22$ & & & \\
\hline $5.000198 \mathrm{E}-03$ & $-7.363851 \mathrm{E}-04$ & & & & $3 / 16 / 996: 11: 24$ & & & \\
\hline $5.000201 \mathrm{E}-03$ & $-7.364064 E-04$ & & & & $3 / 16 / 996: 11: 27$ & & & \\
\hline$-5.000710 \mathrm{E}-03$ & $7.325064 \mathrm{E}-04$ & & & & $3 / 16 / 996: 11: 30$ & & & \\
\hline$-5.000712 \mathrm{E}-03$ & $7.325077 \mathrm{E}-04$ & & & & $3 / 16 / 996: 11: 33$ & & & \\
\hline $5.000207 E-03$ & -7.363997E-04 & & & & $3 / 16 / 996: 11: 36$ & & & \\
\hline $5.000209 E-03$ & $-7.364085 E-04$ & & & & $3 / 16 / 996: 11: 39$ & & & \\
\hline$-5.000710 \mathrm{E}-03$ & $7.325060 \mathrm{E}-04$ & & & & $3 / 16 / 996: 11: 41$ & & & \\
\hline$-5.000711 E-03$ & $7.325118 \mathrm{E}-04$ & & & & $3 / 16 / 996: 11: 44$ & & & \\
\hline $5.000201 \mathrm{E}-03$ & $-7.363990 E-04$ & & & & $3 / 16 / 996: 11: 47$ & & & \\
\hline $5.000195 \mathrm{E}-03$ & $-7.364131 \mathrm{E}-04$ & & & & $3 / 16 / 996: 11: 50$ & & & \\
\hline$-5.000720 \mathrm{E}-03$ & $7.325098 \mathrm{E}-04$ & & & & $3 / 16 / 996: 11: 53$ & & & \\
\hline$-5.000718 \mathrm{E}-03$ & $7.325046 \mathrm{E}-04$ & & & & $3 / 16 / 996: 11: 56$ & & & \\
\hline $5.000199 \mathrm{E}-03$ & $-7.363983 E-04$ & & & & $3 / 16 / 996: 11: 58$ & & & \\
\hline $5.000457 \mathrm{E}-03$ & $7.344525 \mathrm{E}-04$ & 0.1468771 & $1.408990 \mathrm{E}-06$ & 3.672594E-06 & $3 / 16 / 996: 12: 02$ & 4871.355652 & 4.783801866 & \\
\hline & & & & & & & & \\
\hline & & & & & & & & \\
\hline
\end{tabular}


Copper1.xis

\begin{tabular}{|c|c|c|c|c|c|c|c|c|}
\hline Current $(\mathrm{A})$ & Voltage (V) & Res.(ohms) & Std. Dev. on $\Omega$ & Power (W) & Date/time & Cernox (ohms) & Cernox (K) 4th ord & er poly. \\
\hline Sample ID= Cu\#1 & Beam Energy $=$ & $0 \quad$ Time on tar & get $=0$ & & & & & \\
\hline Pre-irradiation resis & vity data at liquid & elium temperature & prior to testing at 1 & $4 \mathrm{GeV}$ & & & & \\
\hline & & & & & & & & \\
\hline 9.999786E-03 & $-1.470755 \mathrm{E}-03$ & & & & $3 / 16 / 996: 12: 55$ & & & \\
\hline$-1.000001 E-02$ & 1.466751E-03 & & & & $3 / 16 / 996: 12: 58$ & & & \\
\hline$-1.000001 \mathrm{E}-02$ & $1.466760 \mathrm{E}-03$ & & & & $3 / 16 / 996: 13: 01$ & & & \\
\hline 9.999790E-03 & $-1.470752 \mathrm{E}-03$ & & & & $3 / 16 / 996: 13: 04$ & & & \\
\hline 9.999787E-03 & $-1.470756 \mathrm{E}-03$ & & & & $3 / 16 / 996: 13: 06$ & & & \\
\hline$-1.000001 E-02$ & $1.466753 \mathrm{E}-03$ & & & & $3 / 16 / 996: 13: 09$ & & & \\
\hline$-1.000001 \mathrm{E}-02$ & $1.466752 \mathrm{E}-03$ & & & & $3 / 16 / 996: 13: 12$ & & & \\
\hline $9.999796 \mathrm{E}-03$ & $-1.470748 \mathrm{E}-03$ & & & & $3 / 16 / 996: 13: 15$ & & & \\
\hline $9.999792 \mathrm{E}-03$ & $-1.470741 \mathrm{E}-03$ & & & & $3 / 16 / 996: 13: 18$ & & & \\
\hline$-1.000001 E-02$ & $1.466781 \mathrm{E}-03$ & & & & $3 / 16 / 996: 13: 21$ & & & \\
\hline$-1.000001 E-02$ & 1.466782t-03 & & & & $3 / 16 / 996: 13: 23$ & & & \\
\hline 9.999792E-03 & $-1.470727 \mathrm{E}-03$ & & & & $3 / 16 / 996: 13: 26$ & & & \\
\hline 9.999787E-03 & $-1.470718 \mathrm{E}-03$ & & & & $3 / 16 / 996: 13: 29$ & & & \\
\hline$-1.000001 \mathrm{E}-02$ & 1.466763E-03 & & & & $3 / 16 / 996: 13: 32$ & & & \\
\hline$-1.000001 E-02$ & 1.466792E-03 & & & & $3 / 16 / 996: 13: 35$ & & & \\
\hline 9.999796E-03 & $-1.470719 \mathrm{E}-03$ & & & & $3 / 16 / 996: 13: 38$ & & & \\
\hline 9.999794E-03 & $-1.470742 \mathrm{E}-03$ & & & & $3 / 16 / 996: 13: 40$ & & & \\
\hline$-1.000001 E-02$ & $1.466786 \mathrm{E}-03$ & & & & $3 / 16 / 996: 13: 43$ & & & \\
\hline$-1.000000 \mathrm{E}-02$ & 1.466794E-03 & & & & $3 / 16 / 996: 13: 46$ & & & \\
\hline 9.999792E-03 & $-1.470721 \mathrm{E}-03$ & & & & $3 / 16 / 996: 13: 49$ & & & \\
\hline 9.999791E-03 & $-1.470728 \mathrm{E}-03$ & & & & $3 / 16 / 996: 13: 52$ & & & \\
\hline$-1.000001 E-02$ & $1.466776 \mathrm{E}-03$ & & & & $3 / 16 / 996: 13: 54$ & & & \\
\hline$-1.000001 E-02$ & 1.466775E-03 & & & & $3 / 16 / 996: 13: 57$ & & & \\
\hline $9.999790 \mathrm{E}-03$ & $-1.470729 \mathrm{E}-03$ & & & & $3 / 16 / 996: 14: 00$ & & & \\
\hline 9.999792E-03 & $-1.470730 \mathrm{E}-03$ & & & & $3 / 16 / 996: 14: 03$ & & & \\
\hline$-1.000001 \mathrm{E}-02$ & $1.466775 \mathrm{E}-03$ & & & & $3 / 16 / 996: 14: 06$ & & & \\
\hline$-1.000000 \mathrm{E}-02$ & $1.466767 \mathrm{E}-03$ & & & & $3 / 16 / 996: 14: 09$ & & & \\
\hline $9.999802 \mathrm{E}-03$ & $-1.470739 \mathrm{E}-03$ & & & & $3 / 16 / 996: 14: 11$ & & & \\
\hline $9.999796 \mathrm{E}-03$ & $-1.470742 E-03$ & & & & $3 / 16 / 996: 14: 14$ & & & \\
\hline$-1.000001 \mathrm{E}-02$ & $1.466768 \mathrm{E}-03$ & & & & $3 / 16 / 996: 14: 17$ & & & \\
\hline$-1.000001 E-02$ & $1.466774 \mathrm{E}-03$ & & & & $3 / 16 / 996: 14: 20$ & & & \\
\hline 9.999799E-03 & $-1.470731 E-03$ & & & & $3 / 16 / 996: 14: 23$ & & & \\
\hline 9.999793E-03 & $-1.470741 E-03$ & & & & $3 / 16 / 996: 14: 26$ & & & \\
\hline$-1.000002 \mathrm{E}-02$ & 1.466756E-03 & & & & $3 / 16 / 996: 14: 28$ & & & \\
\hline$-1.000001 E-02$ & 1.466763E-03 & & & & $3 / 16 / 996: 14: 31$ & & & \\
\hline $9.999798 \mathrm{E}-03$ & $-1.470739 \mathrm{E}-03$ & & & & $3 / 16 / 996: 14: 34$ & & & \\
\hline 9.999794E-03 & $-1.470746 \mathrm{E}-03$ & & & & $3 / 16 / 996: 14: 37$ & & & \\
\hline$-1.000002 E-02$ & $1.466760 \mathrm{E}-03$ & & & & $3 / 16 / 996: 14: 40$ & & & \\
\hline$-1.000001 \mathrm{E}-02$ & 1.466768E-03 & & & & $3 / 16 / 996: 14: 43$ & & & \\
\hline $9.999800 \mathrm{E}-03$ & $-1.470769 \mathrm{E}-03$ & & & & $3 / 16 / 996: 14: 45$ & & & \\
\hline $9.999902 E-03$ & 1.468754E-03 & 0.1468769 & 5.504442E-07 & 1.468754E-05 & $3 / 16 / 996: 14: 49$ & 4869.915504 & 4.784598992 & \\
\hline & & & & & & & & \\
\hline & & & & & & ⿷ & & \\
\hline
\end{tabular}


Copper1.xis

\begin{tabular}{|c|c|c|c|c|c|c|c|c|}
\hline Current (A) & Voltage (V) & Res.(ohms) & Std. Dev. on $\Omega$ & Power (W) & Date/time & Cernox (ohms) & Cernox $(K)$ 4th ord & er poly. \\
\hline Sample ID= Cu\#1 & Beam Energy= & Time on tal & get $=0$ & & & & & \\
\hline \multicolumn{9}{|c|}{ Pre-irradiation resistivity data at liquid helium temperature prior to testing at $1.94 \mathrm{GeV}$} \\
\hline & & & & & & & & \\
\hline 1.499891E-02 & $-2.205240 E-03$ & & & & $3 / 16 / 996: 15: 31$ & & & \\
\hline$-1.500321 \mathrm{E}-02$ & 2.201363E-03 & & & & $3 / 16 / 996: 15: 34$ & & & \\
\hline$-1.500315 E-02$ & $2.201368 \mathrm{E}-03$ & & & & $3 / 16 / 996: 15: 37$ & & & \\
\hline 1.499899E-02 & $-2.205222 E-03$ & & & & $3 / 16 / 996: 15: 40$ & & & \\
\hline 1.499897E-02 & $-2.205265 E-03$ & & & & $3 / 16 / 996: 15: 43$ & & & \\
\hline$-1.500311 \mathrm{E}-02$ & $2.201333 \mathrm{E}-03$ & & & & $3 / 16 / 996: 15: 45$ & & & \\
\hline$-1.500307 \mathrm{E}-02$ & $2.201340 \mathrm{E}-03$ & & & & $3 / 16 / 996: 15: 48$ & & & \\
\hline 1.499903E-02 & $-2.205226 \mathrm{E}-03$ & & & & $3 / 16 / 996: 15: 51$ & & & \\
\hline 1.499904E-02 & $-2.205235 E-03$ & & & & $3 / 16 / 996: 15: 54$ & & & \\
\hline$-1.500308 \mathrm{E}-02$ & 2.201393E-03 & & & & $3 / 16 / 996: 15: 57$ & & & \\
\hline$-1.500311 E-02$ & $2.201386 E-03$ & & & & $3 / 16 / 996: 16: 00$ & & & \\
\hline 1.499906E-02 & $-2.205224 \mathrm{E}-03$ & & & & $3 / 16 / 996: 16: 02$ & & & \\
\hline 1.499909E-02 & $-2.205229 \mathrm{E}-03$ & & & & $3 / 16 / 996: 16: 05$ & & & \\
\hline$-1.500306 \mathrm{E}-02$ & $2.201386 \mathrm{E}-03$ & & & & $3 / 16 / 996: 16: 08$ & & & \\
\hline$-1.500309 \mathrm{E}-02$ & $2.201390 \mathrm{E}-03$ & & & & $3 / 16 / 996: 16: 11$ & & & \\
\hline 1.499904E-02 & $-2.205221 \mathrm{E}-03$ & & & & $3 / 16 / 996: 16: 14$ & & & \\
\hline $1.499902 \mathrm{E}-02$ & $-2.205235 E-03$ & & & & $3 / 16 / 996: 16: 17$ & & & \\
\hline$-1.500305 \mathrm{E}-02$ & $2.201385 E-03$ & & & & $3 / 16 / 996: 16: 19$ & & & \\
\hline$-1.500302 E-02$ & $2.201385 E-03$ & & & & $3 / 16 / 996: 16: 22$ & & & \\
\hline 1.499900E-02 & $-2.205237 \bar{E}-03$ & & & & $3 / 16 / 996: 16: 25$ & & & \\
\hline $1.499903 \mathrm{E}-02$ & $-2.205252 E-03$ & & & & $3 / 16 / 996: 16: 28$ & & & \\
\hline$-1.500310 \mathrm{E}-02$ & $2.201369 \mathrm{E}-03$ & & & & $3 / 16 / 996: 16: 31$ & & & \\
\hline$-1.500309 \mathrm{E}-02$ & $2.201341 \mathrm{E}-03$ & & & & $3 / 16 / 996: 16: 33$ & & & \\
\hline 1.499909E-02 & $-2.205236 \mathrm{E}-03$ & & & & $3 / 16 / 996: 16: 36$ & & & \\
\hline $1.499902 \mathrm{E}-02$ & $-2.205251 E-03$ & & & & $3 / 16 / 996: 16: 39$ & & & \\
\hline$-1.500304 \mathrm{E}-02$ & $2.201343 \mathrm{E}-03$ & & & & $3 / 16 / 996: 16: 42$ & & & \\
\hline$-1.500306 \mathrm{E}-02$ & $2.201338 \mathrm{E}-03$ & & & & $3 / 16 / 996: 16: 45$ & & & \\
\hline 1.499909E-02 & $-2.205242 E-03$ & & & & $3 / 16 / 996: 16: 48$ & & & \\
\hline 1.499907E-02 & $-2.205264 E-03$ & & & & $3 / 16 / 996: 16: 50$ & & & \\
\hline$-1.500310 \mathrm{E}-02$ & $2.201324 \mathrm{E}-03$ & & & & $3 / 16 / 996: 16: 53$ & & & \\
\hline$-1.500306 \mathrm{E}-02$ & 2.201357E-03 & & & & $3 / 16 / 996: 16: 56$ & & & \\
\hline $1.499901 \mathrm{E}-02$ & $-2.205256 E-03$ & & & & $3 / 16 / 996: 16: 59$ & & & \\
\hline $1.499906 \mathrm{E}-02$ & $-2.205272 E-03$ & & & & $3 / 16 / 996: 17: 02$ & & & \\
\hline$-1.500306 \mathrm{E}-02$ & $2.201327 \mathrm{E}-03$ & & & & $3 / 16 / 996: 17: 05$ & & & \\
\hline$-1.500309 \mathrm{E}-02$ & $2.201334 \mathrm{E}-03$ & & & & $3 / 16 / 996: 17: 07$ & & & \\
\hline $1.499901 \mathrm{E}-02$ & $-2.205265 \mathrm{E}-03$ & & & & $3 / 16 / 996: 17: 10$ & & & \\
\hline $1.499903 \mathrm{E}-02$ & $-2.205270 \mathrm{E}-03$ & & & & $3 / 16 / 996: 17: 13$ & & & \\
\hline$-1.500305 E-02$ & $2.201322 \mathrm{E}-03$ & & & & $3 / 16 / 996: 17: 16$ & & & \\
\hline$-1.500305 \mathrm{E}-02$ & $2.201326 \mathrm{E}-03$ & & & & $3 / 16 / 996: 17: 19$ & & & \\
\hline $1.499905 \mathrm{E}-02$ & $-2.205263 E-03$ & & & & $3 / 16 / 996: 17: 22$ & & & \\
\hline $1.500106 \mathrm{E}-02$ & $2.203300 \mathrm{E}-03$ & 0.1468764 & 6.320717E-07 & $3.305173 \mathrm{E}-05$ & $3 / 16 / 996: 17: 26$ & 4868.532384 & 4.785364954 & \\
\hline & & & & & & & & \\
\hline & & & & & & & & \\
\hline
\end{tabular}


Copper1.xls

\begin{tabular}{|c|c|c|c|c|c|c|c|c|}
\hline Current (A) & Voltage (V) & Res.(ohms) & Std. Dev. on $\Omega$ & Power (W) & Date/time & Cernox (ohms) & Cernox $(\mathrm{K})$ 4th ord & er poly. \\
\hline Sample ID = Cu\#1 & Beam Energy= & $1.94 \mathrm{GeV}$ & e on target $=90 \mathrm{~m}$ & utes & & & & \\
\hline First data acquisitic & after irradiation b & $1.94 \mathrm{GeV}$ protons & for 90 minutes at & & & & & \\
\hline & & & & & & & & \\
\hline $5.000209 E-03$ & -7.428750 E-04 & & & & $3 / 16 / 99$ 10:01:50 & & & \\
\hline$-5.000430 \mathrm{E}-03$ & $7.376950 \mathrm{E}-04$ & & & & $3 / 16 / 99$ 10:01:53 & & & \\
\hline$-5.000430 \mathrm{E}-03$ & 7.377207E-04 & & & & $3 / 16 / 9910: 01: 56$ & & & \\
\hline $5.000212 \mathrm{E}-03$ & $-7.428525 \mathrm{E}-04$ & & & & $3 / 16 / 99$ 10:01:59 & & & \\
\hline $5.000210 \mathrm{E}-03$ & $-7.428661 E-04$ & & & & $3 / 16 / 9910: 02: 02$ & & & \\
\hline$-5.000432 E-03$ & $7.377282 \mathrm{E}-04$ & & & & $3 / 16 / 99$ 10:02:04 & & & \\
\hline$-5.000430 \mathrm{E}-03$ & $7.377274 \mathrm{E}-04$ & & & & $3 / 16 / 9910: 02: 07$ & & & \\
\hline $5.000209 \mathrm{E}-03$ & $-7.428499 E-04$ & & & & $3 / 16 / 99$ 10:02:10 & & & \\
\hline $5.000211 \mathrm{E}-03$ & $-7.428520 \mathrm{E}-04$ & & & & $3 / 16 / 9910: 02: 13$ & & & \\
\hline$-5.000435 \mathrm{E}-03$ & $7.377332 \mathrm{E}-04$ & & & & 3/16/99 10:02:16 & & & \\
\hline$-5.000 \overline{0} 32 E-03$ & $7.377370 \bar{E}-04$ & & & & $3 / 16 / 9910: 02: 18$ & & & \\
\hline $5.000209 \mathrm{E}-03$ & $-7.428279 E-04$ & & & & $3 / 16 / 9910: 02: 21$ & & & \\
\hline $5.000209 \mathrm{E}-03$ & $-7.428263 E-04$ & & & & $3 / 16 / 9910: 02: 24$ & & & \\
\hline$-5.000435 \mathrm{E}-03$ & $7.377596 \mathrm{E}-04$ & & & & $3 / 16 / 9910: 02: 27$ & & & \\
\hline$-5.000437 \mathrm{E}-03$ & $7.377405 \mathrm{E}-04$ & & & & $3 / 16 / 9910: 02: 30$ & & & \\
\hline $5.000214 E-03$ & $-7.428134 \mathrm{E}-04$ & & & & $3 / 16 / 99 \quad 10: 02: 33$ & & & \\
\hline $5.000211 \mathrm{E}-03$ & $-7.428223 E-04$ & & & & $3 / 16 / 9910: 02: 35$ & & & \\
\hline$-5.000436 \mathrm{E}-03$ & $7.377616 \mathrm{E}-04$ & & & & $3 / 16 / 9910: 02: 38$ & & & \\
\hline$-5.000436 \mathrm{E}-03$ & $7.377683 \mathrm{E}-04$ & & & & $3 / 16 / 9910: 02: 41$ & & & \\
\hline $5.000213 \mathrm{E}-03$ & $-7.428088 E-04$ & & & & $3 / 16 / 9910: 02: 44$ & & & \\
\hline $5.000209 E-03$ & $-7.428003 E-04$ & & & & $3 / 16 / 9910: 02: 47$ & & & \\
\hline$-5.000437 \mathrm{E}-03$ & $7.377758 \mathrm{E}-04$ & & & & $3 / 16 / 9910: 02: 50$ & & & \\
\hline$-5.000436 \mathrm{E}-03$ & 7.377837E-04 & & & & $3 / 16 / 9910: 02: 52$ & & & \\
\hline $5.000208 \mathrm{E}-03$ & $-7.427954 \mathrm{E}-04$ & & & & $3 / 16 / 99 \quad 10: 02: 55$ & & & \\
\hline $5.000209 \mathrm{E}-03$ & $-7.427922 \mathrm{E}-04$ & & & & $3 / 16 / 9910: 02: 58$ & & & \\
\hline$-5.000444 E-03$ & $7.377907 \mathrm{E}-04$ & & & & 3/16/99 10:03:01 & & & \\
\hline$-5.000445 E-03$ & $7.377883 \mathrm{E}-04$ & & & & 3/16/99 10:03:04 & & & \\
\hline $5.000208 \mathrm{E}-03$ & $-7.427835 \mathrm{E}-04$ & & & & 3/16/99 10:03:07 & & & \\
\hline $5.000209 E-03$ & $-7.427937 \mathrm{E}-04$ & & & & $3 / 16 / 9910: 03: 09$ & & & \\
\hline$-5.000442 E-03$ & $7.378012 \mathrm{E}-04$ & & & & $3 / 16 / 99$ 10:03:12 & & & \\
\hline$-5.000447 E-03$ & $7.378142 \mathrm{E}-04$ & & & & $3 / 16 / 99$ 10:03:15 & & & \\
\hline $5.000206 \mathrm{E}-03$ & $-7.427880 \mathrm{E}-04$ & & & & $3 / 16 / 99$ 10:03:18 & & & \\
\hline $5.000208 \mathrm{E}-03$ & $-7.427751 \mathrm{E}-04$ & & & & $3 / 16 / 99$ 10:03:21 & & & \\
\hline$-5.000447 \mathrm{E}-03$ & $7.377944 \mathrm{E}-04$ & & & & $3 / 16 / 9910: 03: 24$ & & & \\
\hline$-5.000447 \mathrm{E}-03$ & $7.378048 E-04$ & & & & $3 / 16 / 99$ 10:03:26 & & & \\
\hline $5.000210 E-03$ & $-7.427655 \mathrm{E}-04$ & & & & $3 / 16 / 9910: 03: 29$ & & & \\
\hline $5.000210 \mathrm{E}-03$ & $-7.427717 E-04$ & & & & $3 / 16 / 99$ 10:03:32 & & & \\
\hline$-5.000450 \mathrm{E}-03$ & 7.378291E-04 & & & & $3 / 16 / 9910: 03: 35$ & & & \\
\hline$-5.000452 E-03$ & $7.378221 \mathrm{E}-04$ & & & & $3 / 16 / 99$ 10:03:38 & & & \\
\hline $5.000208 \mathrm{E}-03$ & $-7.427630 \mathrm{E}-04$ & & & & $3 / 16 / 9910: 03: 41$ & & & \\
\hline $5.000324 \mathrm{E}-03$ & $7.402900 \mathrm{E}-04$ & 0.1480484 & 1.163321E-06 & $3.701707 \mathrm{E}-06$ & $3 / 16 / 9910: 03: 45$ & 4909.106288 & 4.763057497 & \\
\hline & & & & & & & & \\
\hline
\end{tabular}


Copper1.xls

\begin{tabular}{|c|c|c|c|c|c|c|c|c|}
\hline Current (A) & Voltage (V) & Res.(ohms) & Std. Dev. on $\Omega$ & Power (W) & Date/time & Cernox (ohms) & \multicolumn{2}{|c|}{ Cernox (K) 4th order poly } \\
\hline Sample ID= Cu\#1 & Beam Energy= & $1.94 \mathrm{GeV}$ & \multicolumn{2}{|c|}{ Time on target $=90$ minutes } & & & & \\
\hline \multicolumn{9}{|c|}{ First data acquisition after irradiation by $1.94 \mathrm{GeV}$ protons for 90 minutes at $4.7 \mathrm{~K}$. } \\
\hline & & & & & & & & \\
\hline $9.999874 \mathrm{E}-03$ & $-1.482884 \mathrm{E}-03$ & & & & $3 / 16 / 9910: 04: 51$ & & & \\
\hline$-9.999847 E-03$ & $1.478042 \mathrm{E}-03$ & & & & $3 / 16 / 9910: 04: 54$ & & & \\
\hline$-9.999844 \mathrm{E}-03$ & $1.478035 \mathrm{E}-03$ & & & & $3 / 16 / 9910: 04: 57$ & & & \\
\hline $9.999876 \mathrm{E}-03$ & $-1.482864 \mathrm{E}-03$ & & & & $3 / 16 / 9910: 05: 00$ & & & \\
\hline 9.999874E-03 & $-1.482863 E-03$ & & & & $3 / 16 / 99$ 10:05:02 & & & \\
\hline$-9.999850 E-03$ & 1.478043E-03 & & & & $3 / 16 / 9910: 05: 05$ & & & \\
\hline$-9.999849 E-03$ & $1.478052 E-03$ & & & & $3 / 16 / 9910: 05: 08$ & & & \\
\hline 9.999879E-03 & $-1.482848 E-03$ & & & & $3 / 16 / 99$ 10:05:11 & & & \\
\hline 9.999875E-03 & $-1.482863 E-03$ & & & & $3 / 16 / 99$ 10:05:14 & & & \\
\hline$-9.999847 E-03$ & $1.478066 \mathrm{E}-03$ & & & & $3 / 16 / 99$ 10:05:17 & & & \\
\hline$-9.999842 E-03$ & $1.478064 E-03$ & & & & $3 / 16 / 99$ 10:05:19 & & & \\
\hline 9.999879E-03 & $-1.482847 E-03$ & & & & $3 / 16 / 99 \quad 10: 05: 22$ & & & \\
\hline $9.999872 E-03$ & $-1.482840 E-03$ & & & & $3 / 16 / 9910: 05: 25$ & & & \\
\hline$-9.999848 E-03$ & $1.478078 \mathrm{E}-03$ & & & & $3 / 16 / 9910: 05: 28$ & & & \\
\hline$-9.999845 E-03$ & $1.478078 \mathrm{E}-03$ & & & & $3 / 16 / 9910: 05: 31$ & & & \\
\hline $9.999879 \mathrm{E}-03$ & $-1.482825 E-03$ & & & & $3 / 16 / 9910: 05: 33$ & & & \\
\hline $9.999874 \mathrm{E}-03$ & $-1.482843 E-03$ & & & & $3 / 16 / 9910: 05: 36$ & & & \\
\hline$-9.999852 E-03$ & $1.478077 \mathrm{E}-03$ & & & & $3 / 16 / 9910: 05: 39$ & & & \\
\hline$-9.999848 E-03$ & $1.478074 \mathrm{E}-03$ & & & & $3 / 16 / 9910: 05: 42$ & & & \\
\hline $9.999880 \mathrm{E}-03$ & $-1.482830 E-03$ & & & & $3 / 16 / 9910: 05: 45$ & & & \\
\hline $9.999873 \mathrm{E}-03$ & $-1.482815 E-03$ & & & & $3 / 16 / 9910: 05: 48$ & & & \\
\hline$-9.999856 \mathrm{E}-03$ & $1.478078 \mathrm{E}-03$ & & & & $3 / 16 / 9910: 05: 50$ & & & \\
\hline$-9.999855 E-03$ & $1.478085 \mathrm{E}-03$ & & & & $3 / 16 / 9910: 05: 53$ & & & \\
\hline 9.999871E-03 & $-1.482815 E-03$ & & & & $3 / 16 / 9910: 05: 56$ & & & \\
\hline $9.999869 \mathrm{E}-03$ & $-1.482814 E-03$ & & & & $3 / 16 / 9910: 05: 59$ & & & \\
\hline$-9.999855 E-03$ & $1.478095 \mathrm{E}-03$ & & & & $3 / 16 / 99$ 10:06:02 & & & \\
\hline$-9.999850 E-03$ & 1.478092E-03 & & & & $3 / 16 / 9910: 06: 05$ & & & \\
\hline 9.999873E-03 & $-1.482822 E-03$ & & & & 3/16/99 10:06:07 & & & \\
\hline $9.999872 \mathrm{E}-03$ & $-1.482808 E-03$ & & & & $3 / 16 / 9910: 06: 10$ & & & \\
\hline$-9.999854 E-03$ & $1.478104 \mathrm{E}-03$ & & & & $3 / 16 / 9910: 06: 13$ & & & \\
\hline$-9.999854 E-03$ & 1.478102E-03 & & & & $3 / 16 / 9910: 06: 16$ & & & \\
\hline $9.999870 \mathrm{E}-03$ & $-1.482798 \mathrm{E}-03$ & & & & $3 / 16 / 9910: 06: 19$ & & & \\
\hline $9.999870 \mathrm{E}-03$ & $-1.482792 E-03$ & & & & $3 / 16 / 99$ 10:06:22 & & & \\
\hline$-9.999854 \mathrm{E}-03$ & $1.478106 \mathrm{E}-03$ & & & & $3 / 16 / 9910: 06: 24$ & & & \\
\hline$-9.999853 E-03$ & 1.478112E-03 & & & & $3 / 16 / 9910: 06: 27$ & & & \\
\hline $9.999866 \mathrm{E}-03$ & $-1.482787 E-03$ & & & & $3 / 16 / 9910: 06: 30$ & & & \\
\hline $9.999865 \mathrm{E}-03$ & $-1.482786 \mathrm{E}-03$ & & & & $3 / 16 / 9910: 06: 33$ & & & \\
\hline$-9.999858 E-03$ & 1.478129E-03 & & & & $3 / 16 / 9910: 06: 36$ & & & \\
\hline$-9.999859 \mathrm{E}-03$ & 1.478115E-03 & & & & $3 / 16 / 99$ 10:06:38 & & & \\
\hline $9.999868 \mathrm{E}-03$ & $-1.482776 E-03$ & & & & $3 / 16 / 9910: 06: 41$ & & & \\
\hline 9.999862E-03 & 1.480454E-03 & 0.1480474 & $5.285845 \mathrm{E}-07$ & $1.480425 \mathrm{E}-05$ & $3 / 16 / 9910: 06: 45$ & 4911.533216 & 4.761733726 & \\
\hline & & & & & & & & \\
\hline & & & & & & & & \\
\hline
\end{tabular}


Copper1.xis

\begin{tabular}{|c|c|c|c|c|c|c|c|c|}
\hline Current (A) & Voltage (V) & Res.(ohms) & Std. Dev. on $\Omega$ & Power (W) & \multirow[t]{2}{*}{ Date/time } & \multirow[t]{2}{*}{ Cernox (ohms) } & \multicolumn{2}{|c|}{ Cernox $(\mathrm{K})$ 4th order poly. } \\
\hline Sample ID= Cu\#1 & Beam Energy $=$ & $1.94 \mathrm{GeV}$ & \multicolumn{2}{|c|}{ Time on target $=90$ minutes } & & & & \\
\hline \multicolumn{9}{|c|}{ First data acquisition after irradiation by $1.94 \mathrm{GeV}$ protons for 90 minutes at $4.7 \mathrm{~K}$. } \\
\hline & & & & & & & & \\
\hline 1.499804E-02 & $-2.223103 E-03$ & & & & $3 / 16 / 9910: 07: 47$ & & & \\
\hline$-1.500010 \mathrm{E}-02$ & $2.218081 \mathrm{E}-03$ & & & & $3 / 16 / 9910: 07: 50$ & & & \\
\hline$-1.500010 \mathrm{E}-02$ & $2.218070 \mathrm{E}-03$ & & & & $3 / 16 / 9910: 07: 53$ & & & \\
\hline $1.499816 \mathrm{E}-02$ & $-2.223095 \mathrm{E}-03$ & & & & $3 / 16 / 9910: 07: 56$ & & & \\
\hline $1.499813 \mathrm{E}-02$ & $-2.223087 E-03$ & & & & $3 / 16 / 9910: 07: 59$ & & & \\
\hline$-1.500013 E-02$ & $2.218075 \mathrm{E}-03$ & & & & $3 / 16 / 9910: 08: 01$ & & & \\
\hline$-1.500012 E-02$ & $2.218083 \mathrm{E}-03$ & & & & $3 / 16 / 9910: 08: 04$ & & & \\
\hline $1.499815 \mathrm{E}-02$ & $-2.223097 \mathrm{E}-03$ & & & & $3 / 16 / 9910: 08: 07$ & & & \\
\hline $1.499816 \mathrm{E}-02$ & $-2.223090 \mathrm{E}-03$ & & & & 3/16/99 10:08:10 & & & \\
\hline$-1.500011 E-02$ & $2.218092 \mathrm{E}-03$ & & & & $3 / 16 / 99$ 10:08:13 & & & \\
\hline$-1.50 \overline{0} 0 \overline{1} i \mathrm{E}-0 \mathrm{2}$ & $2.218098 E-03$ & & & & $3 / 16 / 99$ 10:08:16 & & & \\
\hline $1.499818 \mathrm{E}-02$ & $-2.223087 E-03$ & & & & $3 / 16 / 9910: 08: 18$ & & & \\
\hline 1.499823E-02 & $-2.223069 \mathrm{E}-03$ & & & & $3 / 16 / 99$ 10:08:21 & & & \\
\hline$-1.500014 \mathrm{E}-02$ & $2.218110 \mathrm{E}-03$ & & & & 3/16/99 10:08:24 & & & \\
\hline$-1.500011 \mathrm{E}-02$ & $2.218105 \mathrm{E}-03$ & & & & 3/16/99 10:08:27 & & & \\
\hline 1.499822E-02 & $-2.223078 E-03$ & & & & $3 / 16 / 9910: 08: 30$ & & & \\
\hline 1.499819E-02 & $-2.223091 E-03$ & & & & $3 / 16 / 99$ 10:08:32 & & & \\
\hline$-1.500013 E-02$ & $2218113 E-03$ & & & & $3 / 16 / 99$ 10:08:35 & & & \\
\hline$-1.500014 E-02$ & $2.218120 \mathrm{E}-03$ & & & & $3 / 16 / 9910: 08: 38$ & & & \\
\hline 1.499821E-02 & $-2.223082 E-03$ & & & & $3 / 16 / 9910: 08: 41$ & & & \\
\hline 1.499822E-02 & $-2.223070 \mathrm{E}-03$ & & & & $3 / 16 / 9910: 08: 44$ & & & \\
\hline$-1.500016 \mathrm{E}-02$ & $2.218115 \mathrm{E}-03$ & & & & $3 / 16 / 9910: 08: 47$ & & & \\
\hline$-1.500015 E-02$ & $2.218112 \mathrm{E}-03$ & & & & $3 / 16 / 9910: 08: 49$ & & & \\
\hline $1.499824 \mathrm{E}-02$ & $-2.223071 \mathrm{E}-03$ & & & & $3 / 16 / 99$ 10:08:52 & & & \\
\hline $1.499826 \mathrm{E}-02$ & $-2.223058 \mathrm{E}-03$ & & & & $3 / 16 / 99$ 10:08:55 & & & \\
\hline$-1.500019 \mathrm{E}-02$ & $2.218143 \mathrm{E}-03$ & & & & $3 / 16 / 99$ 10:08:58 & & & \\
\hline$-1.500019 \mathrm{E}-02$ & $2.218145 \mathrm{E}-03$ & & & & $3 / 16 / 99$ 10:09:01 & & & \\
\hline 1.499831E-02 & $-2.223059 \mathrm{E}-03$ & & & & $3 / 16 / 99$ 10:09:04 & & & \\
\hline $1.499831 \mathrm{E}-02$ & $-2.223059 E-03$ & & & & $3 / 16 / 99$ 10:09:06 & & & \\
\hline$-1.500014 \mathrm{E}-02$ & $2.218157 \mathrm{E}-03$ & & & & 3/16/99 10:09:09 & & & \\
\hline$-1.500013 E-02$ & $2.218148 \mathrm{E}-03$ & & & & $3 / 16 / 99$ 10:09:12 & & & \\
\hline $1.499832 E-02$ & $-2.223053 \mathrm{E}-03$ & & & & $3 / 16 / 99$ 10:09:15 & & & \\
\hline 1.499835E-02 & $-2.223045 \mathrm{E}-03$ & & & & $3 / 16 / 99$ 10:09:18 & & & \\
\hline$-1.500011 \mathrm{E}-02$ & $2.218162 \mathrm{E}-03$ & & & & $3 / 16 / 99$ 10:09:20 & & & \\
\hline$-1.500010 \mathrm{E}-02$ & $2.218148 \mathrm{E}-03$ & & & & $3 / 16 / 9910: 09: 23$ & & & \\
\hline $1.499832 \mathrm{E}-02$ & $-2.223042 E-03$ & & & & $3 / 16 / 99$ 10:09:26 & & & \\
\hline 1.499833E-02 & $-2.223020 \mathrm{E}-03$ & & & & $3 / 16 / 99$ 10:09:29 & & & \\
\hline$-1.500013 E-02$ & 2.218180 E-03 & & & & 3/16/99 10:09:32 & & & \\
\hline$-1.500011 \mathrm{E}-02$ & $2.218184 E-03$ & & & & $3 / 16 / 99$ 10:09:35 & & & \\
\hline $1.499838 \mathrm{E}-02$ & $-2.223010 \mathrm{E}-03$ & & & & $3 / 16 / 99$ 10:09:37 & & & \\
\hline $1.499918 \mathrm{E}-02$ & 2.220595E-03 & 0.1480477 & 3.763133E-07 & $3.330729 \mathrm{E}-05$ & $3 / 16 / 99$ 10:09:42 & 4907.743266 & 4.763801478 & \\
\hline & & & & & & & & \\
\hline
\end{tabular}


Copper1.xls

\begin{tabular}{|c|c|c|c|c|c|c|c|c|}
\hline Current $(A)$ & Voltage (V) & Res.(ohms) & Std. Dev. on $\Omega$ & Power $(W)$ & Date/time & Cernox (ohms) & \multicolumn{2}{|c|}{ Cernox (K) 4th order poly } \\
\hline Sample ID= Cu\#1 & \multicolumn{4}{|c|}{ Beam Energy $=1.94 \mathrm{GeV} \quad$ Time on target $=2.5$ hours } & & & & \\
\hline \multicolumn{9}{|c|}{ 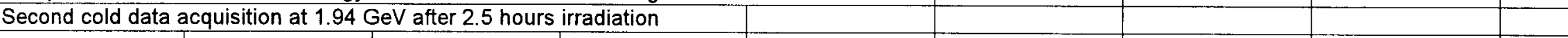 } \\
\hline & & & & & & & & \\
\hline $5.000196 \mathrm{E}-03$ & $-7.470468 E-04$ & & & & $3 / 16 / 9912: 51: 53$ & & & \\
\hline$-5.000491 \mathrm{E}-03$ & $7.417456 \mathrm{E}-04$ & & & & $3 / 16 / 9912: 51: 56$ & & & \\
\hline$-5.000487 \mathrm{E}-03$ & 7.417376E-04 & & & & $3 / 16 / 9912: 51: 59$ & & & \\
\hline $5.000202 \mathrm{E}-03$ & $-7.470551 \mathrm{E}-04$ & & & & $3 / 16 / 9912: 52: 01$ & & & \\
\hline $5.000200 \mathrm{E}-03$ & $-7.470485 E-04$ & & & & $3 / 16 / 99$ 12:52:04 & & & \\
\hline$-5.000495 \mathrm{E}-03$ & 7.417392E-04 & & & & $3 / 16 / 9912: 52: 07$ & & & \\
\hline$-5.000495 \mathrm{E}-03$ & $7.417460 \mathrm{E}-04$ & & & & $3 / 16 / 9912: 52: 10$ & & & \\
\hline $5.000194 \mathrm{E}-03$ & $-7.470509 E-04$ & & & & $3 / 16 / 99$ 12:52:13 & & & \\
\hline $5.000195 \mathrm{E}-03$ & $-7.470485 E-04$ & & & & $3 / 16 / 99$ 12:52:16 & & & \\
\hline$-5.000492 \mathrm{E}-03$ & $7.417441 \mathrm{E}-04$ & & & & $3 / 16 / 99 \quad 12: 52: 18$ & & & \\
\hline$-5.000493 E-03$ & $7.417463 \mathrm{E}-04$ & & & & $3 / 16 / 99$ 12:52:21 & & & \\
\hline $5.000197 \mathrm{E}-03$ & $-7.470398 E-04$ & & & & $3 / 16 / 9912: 52: 24$ & & & \\
\hline 5.000195E-03 & $-7.470312 \mathrm{E}-04$ & & & & $3 / 16 / 99$ 12:52:27 & & & \\
\hline$-5.000499 \mathrm{E}-03$ & $7.417506 \mathrm{E}-04$ & & & & $3 / 16 / 9912: 52: 30$ & & & \\
\hline$-5.000498 \mathrm{E}-03$ & $7.417610 \mathrm{E}-04$ & & & & $3 / 16 / 99$ 12:52:33 & & & \\
\hline 5.000194E-03 & $-7.470298 \mathrm{E}-04$ & & & & $3 / 16 / 9912: 52: 35$ & & & \\
\hline 5.000194E-03 & $-7.470312 \mathrm{E}-04$ & & & & $3 / 16 / 9912: 52: 38$ & & & \\
\hline$-5.000502 \mathrm{E}-03$ & 7.417607E-04 & & & & $3 / 16 / 9912: 52: 41$ & & & \\
\hline$-5.000501 \mathrm{E}-03$ & 7.417655E-04 & & & & $3 / 16 / 99 \quad 12: 52: 44$ & & & \\
\hline $5.000199 \mathrm{E}-03$ & $-7.470332 E-04$ & & & & $3 / 16 / 99$ 12:52:47 & & & \\
\hline $5.000198 \mathrm{E}-03$ & $-7.470354 \mathrm{E}-04$ & & & & $3 / 16 / 9912: 52: 50$ & & & \\
\hline$-5.000506 \mathrm{E}-03$ & $7.417726 \mathrm{E}-04$ & & & & $3 / 16 / 9912: 52: 52$ & & & \\
\hline$-5.000510 \mathrm{E}-03$ & 7.417640E-04 & & & & $3 / 16 / 9912: 52: 55$ & & & \\
\hline 5.000194E-03 & $-7.470257 \mathrm{E}-04$ & & & & $3 / 16 / 99 \quad 12: 52: 58$ & & & \\
\hline $5.000200 \mathrm{E}-03$ & $-7.470295 E-04$ & & & & 3/16/99 12:53:01 & & & \\
\hline$-5.000505 E-03$ & $7.417692 \mathrm{E}-04$ & & & & $3 / 16 / 9912: 53: 04$ & & & \\
\hline$-5.000506 \mathrm{E}-03$ & $7.417758 \mathrm{E}-04$ & & & & $3 / 16 / 99$ 12:53:06 & & & \\
\hline $5.000195 \mathrm{E}-03$ & $-7.470316 \mathrm{E}-04$ & & & & $3 / 16 / 9912: 53: 09$ & & & \\
\hline $5.000193 E-03$ & $-7.470162 \mathrm{E}-04$ & & & & $3 / 16 / 9912: 53: 12$ & & & \\
\hline$-5.000508 \mathrm{E}-03$ & 7.417684E-04 & & & & $3 / 16 / 99$ 12:53:15 & & & \\
\hline$-5.000504 \mathrm{E}-03$ & 7.417554E-04 & & & & $3 / 16 / 9912: 53: 18$ & & & \\
\hline $5.000196 \mathrm{E}-03$ & $-7.470232 \mathrm{E}-04$ & & & & 3/16/99 12:53:21 & & & \\
\hline $5.000194 \mathrm{E}-03$ & $-7.470118 \mathrm{E}-04$ & & & & $3 / 16 / 99$ 12:53:23 & & & \\
\hline$-5.000509 \mathrm{E}-03$ & 7.417644E-04 & & & & $3 / 16 / 99 \quad 12: 53: 26$ & & & \\
\hline$-5.000502 \mathrm{E}-03$ & 7.417793E-04 & & & & $3 / 16 / 99$ 12:53:29 & & & \\
\hline $5.000199 \mathrm{E}-03$ & $-7.470242 E-04$ & & & & $3 / 16 / 99$ 12:53:32 & & & \\
\hline $5.000196 E-03$ & $-7.470229 E-04$ & & & & $3 / 16 / 99$ 12:53:35 & & & \\
\hline$-5.000504 \mathrm{E}-03$ & $7.417843 \mathrm{E}-04$ & & & & $3 / 16 / 99$ 12:53:38 & & & \\
\hline$-5.000506 E-03$ & $7.417800 \mathrm{E}-04$ & & & & $3 / 16 / 9912: 53: 40$ & & & \\
\hline 5.000194E-03 & $-7.470238 \mathrm{E}-04$ & & & & $3 / 16 / 99$ 12:53:43 & & & \\
\hline $5.000348 E-03$ & 7.443967E-04 & 0.1488690 & $9.122245 \mathrm{E}-07$ & $3.722270 \mathrm{E}-06$ & $3 / 16 / 9912: 53: 47$ & 4859.360401 & 4.790454298 & \\
\hline & & & & & & & & \\
\hline & & & & & & & & \\
\hline
\end{tabular}


Copper1.xls

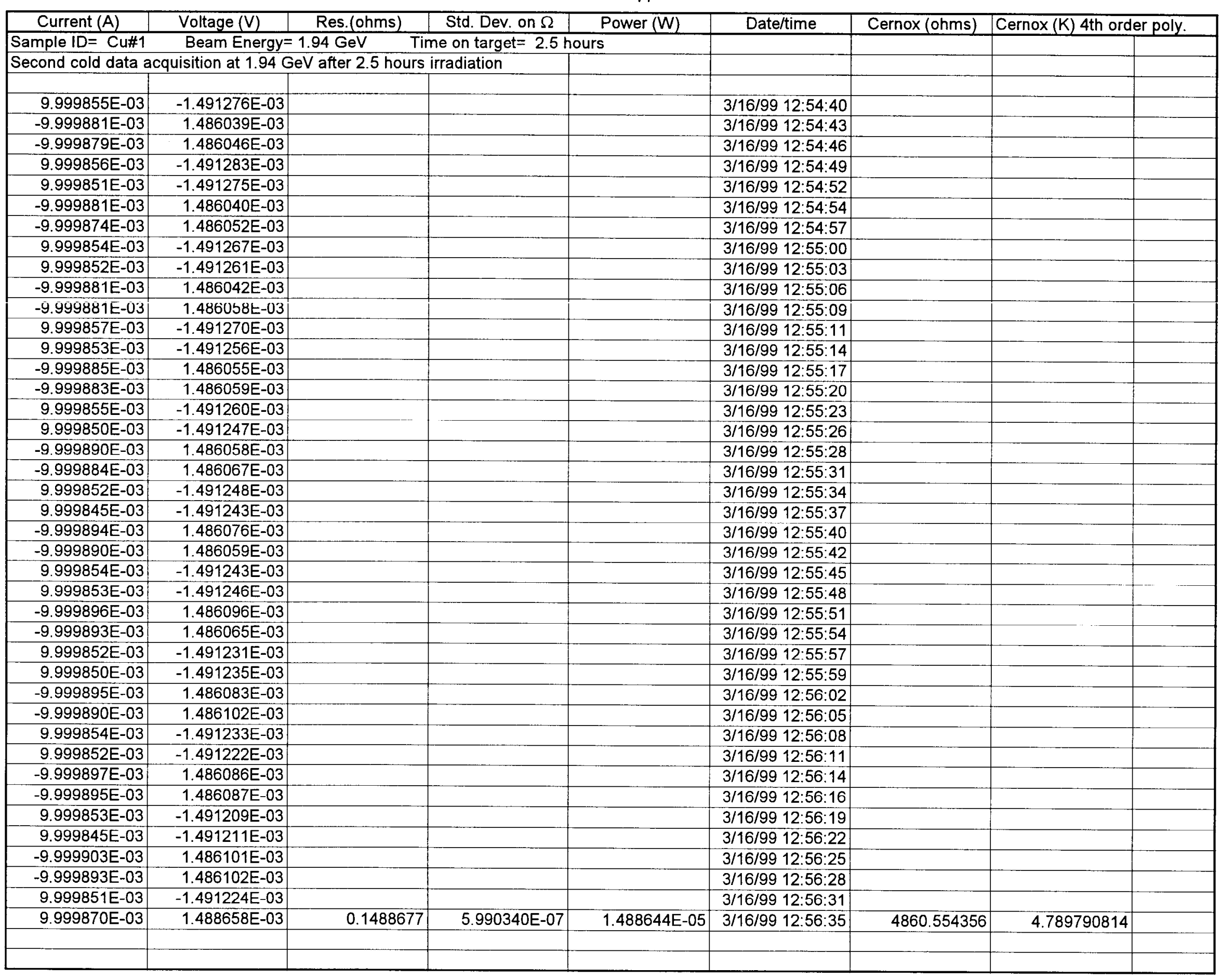


Copper $1 . x \mid s$

\begin{tabular}{|c|c|c|c|c|c|c|c|c|}
\hline Current (A) & Voltage (V) & Res.(ohms) & Std. Dev. on $\Omega$ & Power (W) & Date/time & Cernox (ohms) & \multicolumn{2}{|c|}{ Cernox $(K)$ 4th order poly. } \\
\hline Sample ID= Cu\#1 & \multicolumn{2}{|c|}{ Beam Energy $=1.94 \mathrm{GeV} \quad \mathrm{T}$} & \multicolumn{2}{|c|}{ Time on target $=2.5$ hours } & & & & \\
\hline \multicolumn{9}{|c|}{\begin{tabular}{|l|l} 
Second cold data acquisition at $1.94 \mathrm{GeV}$ after 2.5 hours irradiation &
\end{tabular}} \\
\hline & & & & & & & & \\
\hline 1.499823E-02 & $-2.235628 \mathrm{E}-03$ & & & & $3 / 16 / 9912: 57: 27$ & & & \\
\hline$-1.500081 \mathrm{E}-02$ & $2.230283 E-03$ & & & & $3 / 16 / 9912: 57: 30$ & & & \\
\hline$-1.500084 \mathrm{E}-02$ & $2.230301 \mathrm{E}-03$ & & & & $3 / 16 / 9912: 57: 33$ & & & \\
\hline 1.499826E-02 & $-2.235611 \mathrm{E}-03$ & & & & $3 / 16 / 9912: 57: 36$ & & & \\
\hline 1.499831E-02 & $-2.235603 E-03$ & & & & $3 / 16 / 9912: 57: 38$ & & & \\
\hline$-1.500079 \mathrm{E}-02$ & $2.230279 \mathrm{E}-03$ & & & & $3 / 16 / 9912: 57: 41$ & & & \\
\hline-1.500080 E-02 & $2.230298 \mathrm{E}-03$ & & & & $3 / 16 / 9912: 57: 44$ & & & \\
\hline 1.499830 E-02 & $-2.235624 \mathrm{E}-03$ & & & & $3 / 16 / 9912: 57: 47$ & & & \\
\hline 1.499835E-02 & $-2.235622 \mathrm{E}-03$ & & & & $3 / 16 / 9912: 57: 50$ & & & \\
\hline$-1.500078 E-02$ & $2.230289 \mathrm{E}-03$ & & & & $3 / 16 / 9912: 57: 53$ & & & \\
\hline$-1.500081 E-02$ & $2.2302 / 4 t-03$ & & & & $3 / 16 / 9912: 57: 55$ & & & \\
\hline 1.499834E-02 & $-2.235615 \mathrm{E}-03$ & & & & $3 / 16 / 9912: 57: 58$ & & & \\
\hline 1.499837E-02 & $-2.235597 \mathrm{E}-03$ & & & & $3 / 16 / 9912: 58: 01$ & & & \\
\hline$-1.500082 \mathrm{E}-02$ & $2.230300 \mathrm{E}-03$ & & & & $3 / 16 / 9912: 58: 04$ & & & \\
\hline$-1.500083 \mathrm{E}-02$ & $2.230288 \mathrm{E}-03$ & & & & 3/16/99 12:58:07 & & & \\
\hline $1.499835 \mathrm{E}-02$ & $-2.235595 \mathrm{E}-03$ & & & & $3 / 16 / 9912: 58: 10$ & & & \\
\hline 1.499832E-02 & $-2.235601 \mathrm{E}-03$ & & & & $3 / 16 / 9912: 58: 12$ & & & \\
\hline$-1.500082 \mathrm{E}-02$ & $2.230296 \mathrm{E}-03$ & & & & $3 / 16 / 9912: 58: 15$ & & & \\
\hline$-1.500085 E-02$ & $2.230303 E-03$ & & & & $3 / 16 / 9912: 58: 18$ & & & \\
\hline $1.499834 \mathrm{E}-02$ & $-2.235590 \mathrm{E}-03$ & & & & $3 / 16 / 9912: 58: 21$ & & & \\
\hline 1.499836E-02 & $-2.235586 \mathrm{E}-03$ & & & & $3 / 16 / 9912: 58: 24$ & & & \\
\hline$-1.500081 E-02$ & $2.230315 \mathrm{E}-03$ & & & & $3 / 16 / 9912: 58: 27$ & & & \\
\hline$-1.500082 \mathrm{E}-02$ & $2.230296 \mathrm{E}-03$ & & & & $3 / 16 / 9912: 58: 29$ & & & \\
\hline $1.499836 \mathrm{E}-02$ & $-2.235583 \mathrm{E}-03$ & & & & $3 / 16 / 9912: 58: 32$ & & & \\
\hline $1.499836 \mathrm{E}-02$ & $-2.235603 E-03$ & & & & $3 / 16 / 9912.58: 35$ & & & \\
\hline$-1.500081 E-02$ & $2.230318 \mathrm{E}-03$ & & & & $3 / 16 / 9912: 58: 38$ & & & \\
\hline$-1.500083 \mathrm{E}-02$ & $2.230311 \mathrm{E}-03$ & & & & $3 / 16 / 9912: 58: 41$ & & & \\
\hline $1.499832 \mathrm{E}-02$ & $-2.235598 \mathrm{E}-03$ & & & & $3 / 16 / 9912: 58: 44$ & & & \\
\hline 1.499836E-02 & $-2.235589 \mathrm{E}-03$ & & & & $3 / 16 / 9912: 58: 46$ & & & \\
\hline$-1.500082 \mathrm{E}-02$ & $2.230319 \mathrm{E}-03$ & & & & $3 / 16 / 9912: 58: 49$ & & & \\
\hline$-1.500084 E-02$ & $2.230316 \mathrm{E}-03$ & & & & $3 / 16 / 9912: 58: 52$ & & & \\
\hline $1.499834 \mathrm{E}-02$ & $-2.235576 \mathrm{E}-03$ & & & & $3 / 16 / 9912: 58: 55$ & & & \\
\hline $1.499834 \mathrm{E}-02$ & $-2.235585 \mathrm{E}-03$ & & & & $3 / 16 / 9912: 58: 58$ & & & \\
\hline$-1.500084 \mathrm{E}-02$ & $2.230332 E-03$ & & & & $3 / 16 / 9912: 59: 00$ & & & \\
\hline$-1.500084 E-02$ & $2.230345 E-03$ & & & & $3 / 16 / 99$ 12:59:03 & & & \\
\hline $1.499836 \mathrm{E}-02$ & $-2.235588 \mathrm{E}-03$ & & & & $3 / 16 / 99$ 12:59:06 & & & \\
\hline $1.499834 \mathrm{E}-02$ & $-2.235589 \mathrm{E}-03$ & & & & $3 / 16 / 99$ 12:59:09 & & & \\
\hline$-1.500086 \mathrm{E}-02$ & $2.230336 \mathrm{E}-03$ & & & & $3 / 16 / 9912: 59: 12$ & & & \\
\hline$-1.500086 \mathrm{E}-02$ & $2.230333 \mathrm{E}-03$ & & & & $3 / 16 / 9912: 59: 15$ & & & \\
\hline $1.499842 \mathrm{E}-02$ & $-2.235565 E-03$ & & & & $3 / 16 / 99$ 12:59:17 & & & \\
\hline $1.499958 \mathrm{E}-02$ & $2.232952 \mathrm{E}-03$ & 0.1488676 & 5.665143E-07 & 3.349343E-05 & $3 / 16 / 99$ 12:59:22 & 4860.619498 & 4.789754623 & \\
\hline & & & & & & & & \\
\hline & & & & & & & & \\
\hline
\end{tabular}


Copper1.xls

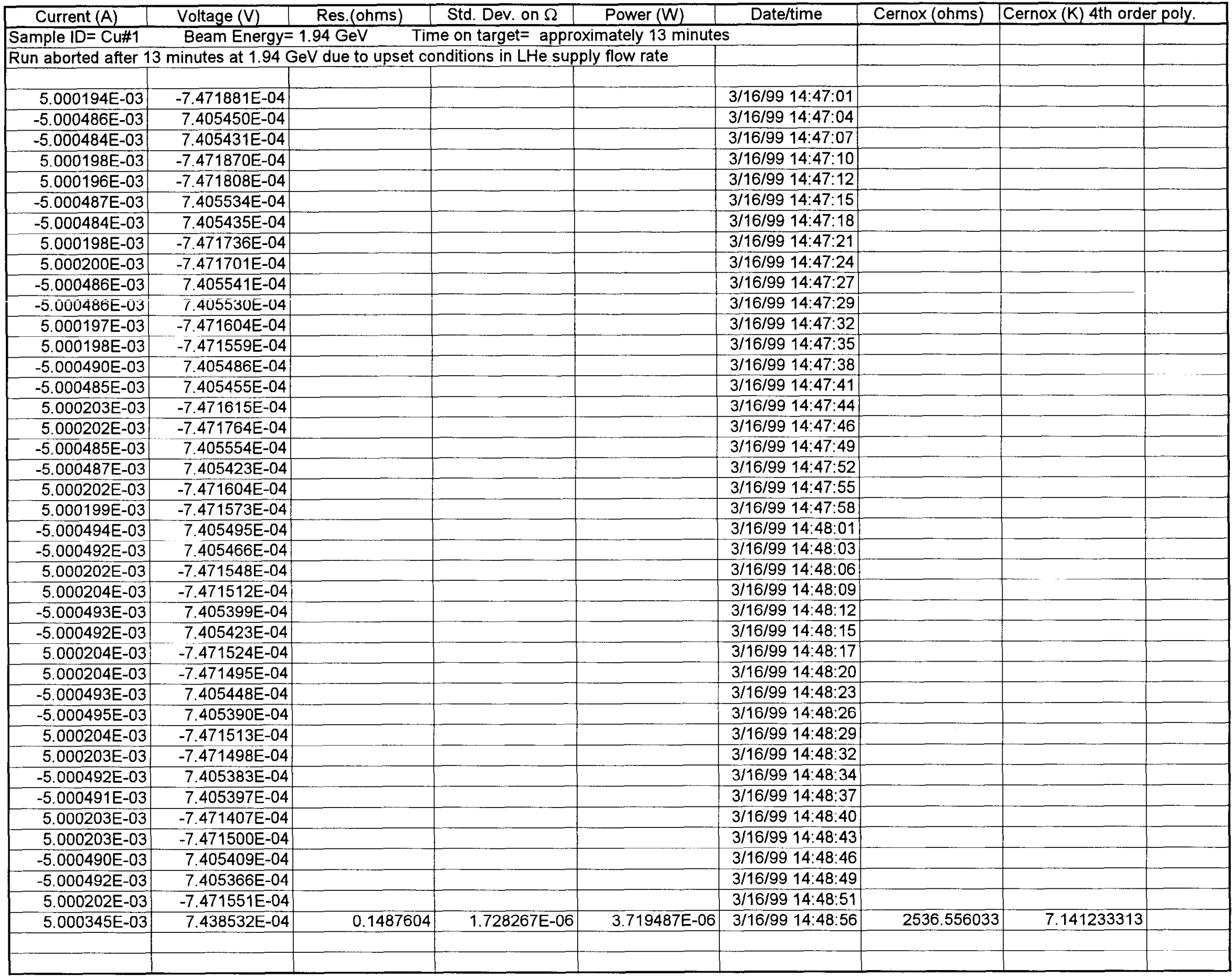


Copper1.xls

\begin{tabular}{|c|c|c|c|c|c|c|c|c|}
\hline Current $(A)$ & Voltage (V) & Res.(ohms) & Std. Dev. on $\Omega$ & Power (W) & Date/time & Cernox (ohms) & Cernox (K) 4th ord & er poly. \\
\hline Sample ID = Cu\#1 & Beam Energy= & $94 \mathrm{GeV}$ & e on target $=$ appro & mately 13 minute & & & & \\
\hline Run aborted after 1 & minutes at 1.94 & $V$ due to upset co & ditions in LHe sup & y flow rate & & & & \\
\hline & & & & & & & & \\
\hline $9.999860 \mathrm{E}-03$ & $-1.490839 \mathrm{E}-03$ & & & & $3 / 16 / 9914: 49: 47$ & & & \\
\hline$-9.999864 \mathrm{E}-03$ & $1.484242 \mathrm{E}-03$ & & & & $3 / 16 / 9914: 49: 50$ & & & \\
\hline$-9.999858 E-03$ & 1.484247E-03 & & & & $3 / 16 / 9914: 49: 53$ & & & \\
\hline 9.999855E-03 & $-1.490839 \mathrm{E}-03$ & & & & $3 / 16 / 9914: 49: 56$ & & & \\
\hline $9.999853 E-03$ & $-1.490834 \mathrm{E}-03$ & & & & $3 / 16 / 9914: 49: 58$ & & & \\
\hline$-9.999868 E-03$ & $1.484248 E-03$ & & & & $3 / 16 / 9914: 50: 01$ & & & \\
\hline$-9.999864 \mathrm{E}-03$ & $1.484250 \mathrm{E}-03$ & & & & $3 / 16 / 9914: 50: 04$ & & & \\
\hline $9.999858 \mathrm{E}-03$ & $-1.490817 E-03$ & & & & $3 / 16 / 9914: 50: 07$ & & & \\
\hline $9.999856 E-03$ & $-1.490820 E-03$ & & & & $3 / 16 / 99$ 14:50:10 & & & \\
\hline$-9.999861 E-03$ & $1.484252 \mathrm{E}-03$ & & & & $3 / 16 / 9914: 50: 13$ & & & \\
\hline$-9.999858 E-03$ & $1.484243 \mathrm{E}-03$ & & & & $3 / 16 / 9914: 50: 15$ & & & \\
\hline $9.999858 E-03$ & $-1.490814 \mathrm{E}-03$ & & & & $3 / 16 / 9914: 50: 18$ & & & \\
\hline $9.999854 \mathrm{E}-03$ & $-1.490810 E-03$ & & & & $3 / 16 / 9914: 50: 21$ & & & \\
\hline$-9.999866 \mathrm{E}-03$ & 1.484245E-03 & & & & $3 / 16 / 9914: 50: 24$ & & & \\
\hline$-9.999863 \mathrm{E}-03$ & 1.484262E-03 & & & & $3 / 16 / 9914: 50: 27$ & & & \\
\hline $9.999860 \mathrm{E}-03$ & $-1.490806 E-03$ & & & & $3 / 16 / 9914: 50: 30$ & & & \\
\hline 9.999855E-03 & $-1.4 \overline{490802 E-03}$ & & & & $3 / 16 / 9914: 50: 32$ & & & \\
\hline$-9.999863 E-03$ & $1.484254 \mathrm{E}-03$ & & & & $3 / 16 / 9914: 50: 35$ & & & \\
\hline$-9.999862 \mathrm{E}-03$ & $1.484262 \mathrm{E}-03$ & & & & $3 / 16 / 9914: 50: 38$ & & & \\
\hline 9.999858E-03 & $-1.490784 \mathrm{E}-03$ & & & & $3 / 16 / 9914: 50: 41$ & & & \\
\hline 9.999854E-03 & $-1.490788 \mathrm{E}-03$ & & & & $3 / 16 / 9914: 50: 44$ & & & \\
\hline$-9.999865 E-03$ & $1.484253 \mathrm{E}-03$ & & & & $3 / 16 / 9914: 50: 47$ & & & \\
\hline$-9.999861 \mathrm{E}-03$ & $1.484255 \mathrm{E}-03$ & & & & $3 / 16 / 9914: 50: 49$ & & & \\
\hline 9.999857E-03 & $-1.490786 E-03$ & & & & $3 / 16 / 9914: 50: 52$ & & & \\
\hline 9.999855E-03 & $-1.490783 E-03$ & & & & $3 / 16 / 9914: 50.55$ & & & \\
\hline$-9.999866 \mathrm{E}-03$ & $1.484271 E-03$ & & & & $3 / 16 / 9914: 50: 58$ & & & \\
\hline$-9.999868 E-03$ & 1.484269E-03 & & & & $3 / 16 / 9914: 51: 01$ & & & \\
\hline $9.999858 \mathrm{E}-03$ & $-1.490775 \mathrm{E}-03$ & & & & $3 / 16 / 9914: 51: 03$ & & & \\
\hline 9.999854E-03 & $-1.490775 E-03$ & & & & $3 / 16 / 9914: 51: 06$ & & & \\
\hline$-9.999871 \mathrm{E}-03$ & $1.484274 E-03$ & & & & $3 / 16 / 9914: 51: 09$ & & & \\
\hline$-9.999868 \mathrm{E}-03$ & $1.484271 \mathrm{E}-03$ & & & & $3 / 16 / 9914: 51: 12$ & & & \\
\hline 9.999859E-03 & $-1.490779 \mathrm{E}-03$ & & & & $3 / 16 / 9914: 51: 15$ & & & \\
\hline $9.999855 \mathrm{E}-03$ & $-1.490769 \mathrm{E}-03$ & & & & $3 / 16 / 9914: 51: 18$ & & & \\
\hline$-9.999869 \mathrm{E}-03$ & $1.484264 \mathrm{E}-03$ & & & & $3 / 16 / 9914: 51: 20$ & & & \\
\hline$-9.999867 \mathrm{E}-03$ & 1.484278E-03 & & & & $3 / 16 / 9914: 51: 23$ & & & \\
\hline $9.999859 \mathrm{E}-03$ & $-1.490754 \mathrm{E}-03$ & & & & $3 / 16 / 99$ 14:51:26 & & & \\
\hline $9.99985 \overline{4 E-03}$ & $-1.490747 \mathrm{E}-03$ & & & & $3 / 16 / 99$ 14:51:29 & & & \\
\hline$-9.999871 \mathrm{E}-03$ & $1.484265 \mathrm{E}-03$ & & & & $3 / 16 / 9914: 51: 32$ & & & \\
\hline$-9.999870 \mathrm{E}-03$ & $1.484279 E-03$ & & & & $3 / 16 / 9914: 51: 35$ & & & \\
\hline $9.999858 \mathrm{E}-03$ & $-1.490741 \mathrm{E}-03$ & & & & $3 / 16 / 9914: 51: 37$ & & & \\
\hline 9.999861E-03 & $1.487526 \mathrm{E}-03$ & 0.1487547 & $9.903400 \mathrm{E}-07$ & $1.487490 \mathrm{E}-05$ & $3 / 16 / 99 \quad 14: 51: 42$ & 2502.857794 & 7.205578947 & \\
\hline & & & & & & & & \\
\hline & & & & & & & & \\
\hline
\end{tabular}


Copper1.xls

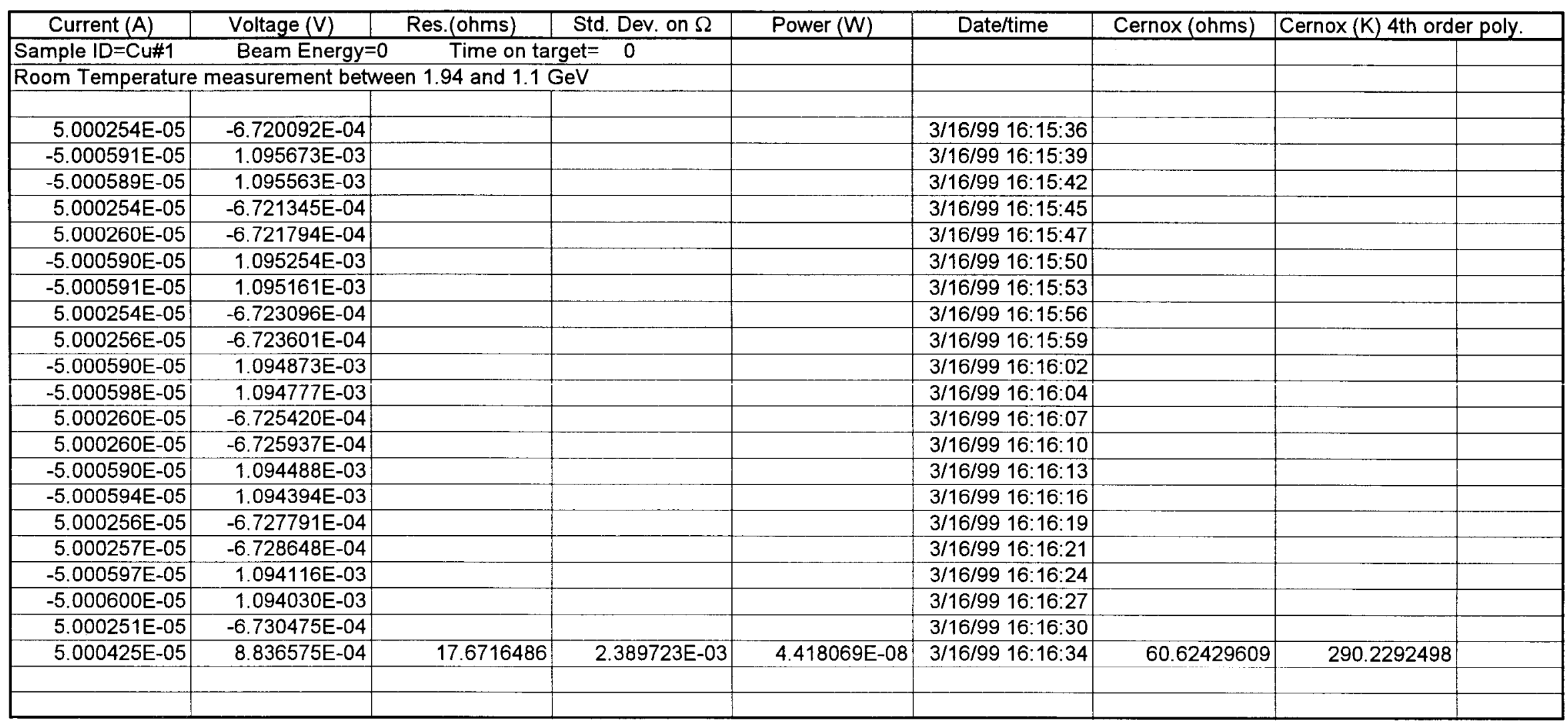


Copper1.xls

\begin{tabular}{|c|c|c|c|c|c|c|c|c|}
\hline Current (A) & Voltage (V) & Res.(ohms) & Std. Dev. on $\Omega$ & Power (W) & Date/time & Cernox (ohms) & \multicolumn{2}{|c|}{ Cernox $(K)$ 4th order poly } \\
\hline \multirow{2}{*}{\multicolumn{9}{|c|}{\begin{tabular}{|lccc} 
Sample ID $=$ Cu\#1 & Beam Energy $=0$ & Time on target $=$ & 0 \\
Room Temperature measurement between 1.94 and $1.1 \mathrm{GeV}$ &
\end{tabular}}} \\
\hline & & & & & & & & \\
\hline \\
\hline 5.000224E-04 & $-8.624823 \mathrm{E}-03$ & & & & $3 / 16 / 9916: 17: 20$ & & & \\
\hline$-5.000730 \mathrm{E}-04$ & $9.042940 \mathrm{E}-03$ & & & & $3 / 16 / 99$ 16:17:23 & & & \\
\hline$-5.000730 \mathrm{E}-04$ & $9.042917 \mathrm{E}-03$ & & & & 3/16/99 16:17:26 & & & \\
\hline $5.000221 \mathrm{E}-04$ & $-8.625301 E-03$ & & & & $3 / 16 / 99$ 16:17:29 & & & \\
\hline $5.000230 \mathrm{E}-04$ & $-8.625524 E-03$ & & & & $3 / 16 / 9916: 17: 32$ & & & \\
\hline$-5.000727 \mathrm{E}-04$ & $9.042854 \mathrm{E}-03$ & & & & $3 / 16 / 9916: 17: 34$ & & & \\
\hline$-5.000720 \mathrm{E}-04$ & $9.042903 \mathrm{E}-03$ & & & & $3 / 16 / 9916: 17: 37$ & & & \\
\hline $5.000231 \mathrm{E}-04$ & $-8.626126 \mathrm{E}-03$ & & & & $3 / 16 / 99$ 16:17:40 & & & \\
\hline $5.000230 \mathrm{E}-04$ & $-8.626334 E-03$ & & & & $3 / 16 / 9916: 17: 43$ & & & \\
\hline$-5.000723 E-04$ & $9.042989 \mathrm{E}-03$ & & & & $3 / 16 / 99$ 16:17:46 & & & \\
\hline$-5.000725 E-04$ & $9.043043 E-03$ & & & & $3 / 16 / 9916: 17: 49$ & & & \\
\hline $5.000221 \mathrm{E}-04$ & $-8.627069 \mathrm{E}-03$ & & & & $3 / 16 / 9916: 17: 51$ & & & \\
\hline $5.000219 \mathrm{E}-04$ & $-8.627347 \mathrm{E}-03$ & & & & $3 / 16 / 9916: 17: 54$ & & & \\
\hline$-5.000726 \mathrm{E}-04$ & $9.043206 \mathrm{E}-03$ & & & & $3 / 16 / 9916: 17: 57$ & & & \\
\hline$-5.000733 \mathrm{E}-04$ & $9.043311 \mathrm{E}-03$ & & & & 3/16/99 16:18:00 & & & \\
\hline $5.000227 \mathrm{E}-04$ & $-8.628155 E-03$ & & & & 3/16/99 16:18:03 & & & \\
\hline 5.000223E-04 & $-8.628428 \mathrm{E}-03$ & & & & 3/16/99 16:18:06 & & & \\
\hline$-5.000723 \mathrm{E}-04$ & $9.043629 \mathrm{E}-03$ & & & & $3 / 16 / 99$ 16:18:08 & & & \\
\hline$-5.000728 \mathrm{E}-04$ & $9.043740 \mathrm{E}-03$ & & & & 3/16/99 16:18:11 & & & \\
\hline $5.000235 \mathrm{E}-04$ & $-8.629371 E-03$ & & & & $3 / 16 / 99$ 16:18:14 & & & \\
\hline $5.000476 \mathrm{E}-04$ & $8.835000 \mathrm{E}-03$ & 17.6683177 & $1.688824 \mathrm{E}-03$ & $4.418703 \mathrm{E}-06$ & $3 / 16 / 99$ 16:18:18 & 60.61359529 & 290.2886144 & \\
\hline & & & & & & & & \\
\hline
\end{tabular}


Copper1.x/s

\begin{tabular}{|c|c|c|c|c|c|c|c|c|}
\hline Current $(A)$ & Voltage (V) & Res.(ohms) & Std. Dev. on $\Omega$ & Power (W) & Date/time & Cernox (ohms) & Cernox $(K)$ 4th ord & er poly. \\
\hline Sample ID= Cu\#1 & Beam Energy $=$ & Time 0 & $n$ target $=$ none & & & & & \\
\hline Room temperature & ata after over-nigh & warm up after 1.5 & $4 \mathrm{GeV}$ & & & & & \\
\hline & & & & & & & & \\
\hline $5.000223 E-05$ & $-8.990694 \mathrm{E}-04$ & & & & $3 / 17 / 998: 37: 54$ & & & \\
\hline$-5.000406 \mathrm{E}-05$ & $8.984294 \mathrm{E}-04$ & & & & $3 / 17 / 998: 37: 57$ & & & \\
\hline$-5.000404 E-05$ & $8.984438 E-04$ & & & & 3/17/99 8:38:00 & & & \\
\hline $5.000227 \mathrm{E}-05$ & $-8.990719 \mathrm{E}-04$ & & & & $3 / 17 / 998: 38: 03$ & & & \\
\hline $5.000228 \mathrm{E}-05$ & $-8.990641 E-04$ & & & & $3 / 17 / 998: 38: 06$ & & & \\
\hline$-5.000409 E-05$ & $8.984534 \mathrm{E}-04$ & & & & $3 / 17 / 998: 38: 09$ & & & \\
\hline$-5.000406 \mathrm{E}-05$ & $8.984469 \mathrm{E}-04$ & & & & $3 / 17 / 998: 38: 11$ & & & \\
\hline $5.000228 \mathrm{E}-05$ & $-8.990467 E-04$ & & & & $3 / 17 / 998: 38: 14$ & & & \\
\hline $5.000231 \mathrm{E}-05$ & $-8.990522 E-04$ & & & & $3 / 17 / 998: 38: 17$ & & & \\
\hline$-5.000408 \mathrm{E}-05$ & $8.984529 \mathrm{E}-04$ & & & & $3 / 17 / 998: 38: 20$ & & & \\
\hline$-5.000406 \mathrm{E}-05$ & $8.984569 \mathrm{E}-04$ & & & & $3 / 17 / 998: 38: 23$ & & & \\
\hline $5.000229 \mathrm{E}-05$ & $-8.990446 \mathrm{E}-04$ & & & & $3 / 17 / 998: 38: 26$ & & & \\
\hline $5.000229 E-05$ & $-8.990396 \mathrm{E}-04$ & & & & $3 / 17 / 998: 38: 28$ & & & \\
\hline$-5.000410 \mathrm{E}-05$ & 8.984520E-04 & & & & $3 / 17 / 998: 38: 31$ & & & \\
\hline$-5.000409 E-05$ & $8.984674 \mathrm{E}-04$ & & & & $3 / 17 / 998: 38: 34$ & & & \\
\hline $5.000230 \mathrm{E}-05$ & $-8.990507 E-04$ & & & & $3 / 17 / 998: 38: 37$ & & & \\
\hline $5.000231 \mathrm{E}-05$ & $-8.990614 \mathrm{E}-04$ & & & & 3/17/99 8:38:40 & & & \\
\hline$-5.000412 \mathrm{E}-05$ & $8.984741 \mathrm{E}-04$ & & & & $3 / 17 / 998: 38: 42$ & & & \\
\hline$-5.000411 \mathrm{E}-05$ & $8.984635 E-04$ & & & & $3 / 17 / 998: 38: 45$ & & & \\
\hline $5.000233 E-05$ & $-8.990528 \mathrm{E}-04$ & & & & $3 / 17 / 998: 38: 48$ & & & \\
\hline $5.000232 E-05$ & $-8.990385 E-04$ & & & & $3 / 17 / 998: 38: 51$ & & & \\
\hline$-5.000415 E-05$ & $8.984668 \mathrm{E}-04$ & & & & $3 / 17 / 99$ 8:38:54 & & & \\
\hline$-5.000413 \mathrm{E}-05$ & $8.984652 E-04$ & & & & $3 / 17 / 998: 38: 57$ & & & \\
\hline $5.000233 \mathrm{E}-05$ & $-8.990335 E-04$ & & & & $3 / 17 / 998: 38: 59$ & & & \\
\hline $5.000233 E-05$ & $-8.990388 E-04$ & & & & $3 / 17 / 998: 39: 02$ & & & \\
\hline$-5.000416 \mathrm{E}-05$ & 8.984737E-04 & & & & 3/17/99 8:39:05 & & & \\
\hline$-5.000415 \mathrm{E}-05$ & $8.984696 \mathrm{E}-04$ & & & & $3 / 17 / 998: 39: 08$ & & & \\
\hline $5.000236 \mathrm{E}-05$ & $-8.990204 \mathrm{E}-04$ & & & & $3 / 17 / 998: 39: 11$ & & & \\
\hline $5.000237 \mathrm{E}-05$ & $-8.990143 \mathrm{E}-04$ & & & & $3 / 17 / 998: 39: 14$ & & & \\
\hline$-5.000414 \mathrm{E}-05$ & $8.984865 \mathrm{E}-04$ & & & & $3 / 17 / 998: 39: 16$ & & & \\
\hline$-5.000416 \mathrm{E}-05$ & $8.984989 \mathrm{E}-04$ & & & & $3 / 17 / 998: 39: 19$ & & & \\
\hline 5.000239E-05 & $-8.990285 \mathrm{E}-04$ & & & & $3 / 17 / 998: 39: 22$ & & & \\
\hline $5.000236 \mathrm{E}-05$ & $-8.990263 \mathrm{E}-04$ & & & & $3 / 17 / 998: 39: 25$ & & & \\
\hline$-5.000418 \mathrm{E}-05$ & $8.984861 \mathrm{E}-04$ & & & & $3 / 17 / 99$ 8:39:28 & & & \\
\hline$-5.000420 \mathrm{E}-05$ & $8.984846 \mathrm{E}-04$ & & & & $3 / 17 / 998: 39: 30$ & & & \\
\hline $5.000233 E-05$ & $-8.990078 \mathrm{E}-04$ & & & & $3 / 17 / 998: 39: 33$ & & & \\
\hline 5.000233E-05 & $-8.990122 \mathrm{E}-04$ & & & & $3 / 17 / 998: 39: 36$ & & & \\
\hline$-5.000412 \mathrm{E}-05$ & $8.984990 \mathrm{E}-04$ & & & & $3 / 17 / 998: 39: 39$ & & & \\
\hline$-5.000416 \mathrm{E}-05$ & $8.985112 \mathrm{E}-04$ & & & & $3 / 17 / 998: 39: 42$ & & & \\
\hline $5.000232 E-05$ & $-8.989938 \mathrm{E}-04$ & & & & $3 / 17 / 99$ 8:39:45 & & & \\
\hline $5.000322 \mathrm{E}-05$ & 8.987537E-04 & 17.9739182 & 1.187639E-04 & 4.494053E-08 & $3 / 17 / 998: 39: 49$ & 60.59897298 & 290.3697699 & \\
\hline & & & & & & & & \\
\hline & & & & & & & & \\
\hline
\end{tabular}


Copper1.xis

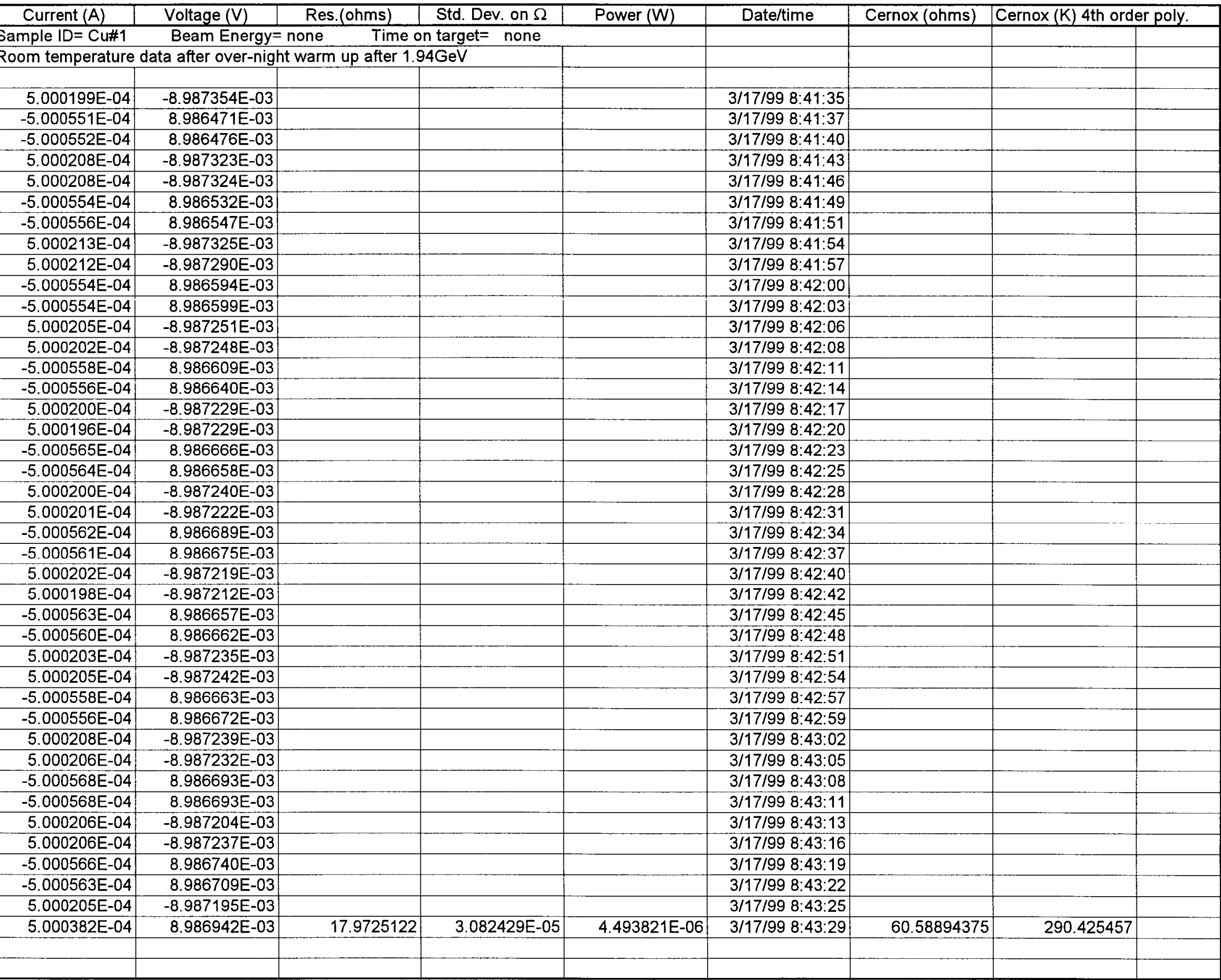


Copper $1 . x \mid s$

\begin{tabular}{|c|c|c|c|c|c|c|c|c|}
\hline Current $(A)$ & Voltage (V) & Res.(ohms) & Std. Dev. on $\Omega$ & Power (W) & Date/time & Cernox (ohms) & \multicolumn{2}{|c|}{ Cernox (K) 4th order poly. } \\
\hline Sample ID= Cu\#1 & \multicolumn{2}{|c|}{ Beam Energy $=$ pre $-1.1 \mathrm{GeV}$} & Time on target $=0$ & & & & & \\
\hline \multicolumn{9}{|c|}{ This is the cold data acquisition at $4.72 \mathrm{~K}$ prior to irradiation at $1.1 \mathrm{GeV}$} \\
\hline & & & & & & & & \\
\hline $5.000180 \mathrm{E}-03$ & $-7.379192 \mathrm{E}-04$ & & & & $3 / 17 / 9910: 01: 09$ & & & \\
\hline$-5.000678 \mathrm{E}-03$ & 7.340835E-04 & & & & $3 / 17 / 9910: 01: 12$ & & & \\
\hline$-5.000674 E-03$ & 7.340877E-04 & & & & $3 / 17 / 9910: 01: 15$ & & & \\
\hline $5.000176 \mathrm{E}-03$ & $-7.379201 \mathrm{E}-04$ & & & & 3/17/99 10:01:18 & & & \\
\hline 5.000177E-03 & $-7.379383 E-04$ & & & & $3 / 17 / 99$ 10:01:20 & & & \\
\hline$-5.000676 \mathrm{E}-03$ & $7.341012 \mathrm{E}-04$ & & & & $3 / 17 / 99$ 10:01:23 & & & \\
\hline$-5.000682 E-03$ & 7.340887E-04 & & & & $3 / 17 / 9910: 01: 26$ & & & \\
\hline $5.000180 \mathrm{E}-03$ & $-7.379286 \mathrm{E}-04$ & & & & $3 / 17 / 99$ 10:01:29 & & & \\
\hline $5.000180 \mathrm{E}-03$ & $-7.379318 E-04$ & & & & $3 / 17 / 99$ 10:01:32 & & & \\
\hline$-5.000678 \mathrm{E}-03$ & $7.340950 \mathrm{E}-04$ & & & & $3 / 17 / 99$ 10:01:35 & & & \\
\hline$-5.000677 \mathrm{E}-03$ & $7.340910 \mathrm{E}-04$ & & & & $3 / 17 / 9910: 01: 37$ & & & \\
\hline 5.000185E-03 & $-7.379306 \mathrm{E}-04$ & & & & $3 / 17 / 9910: 01: 40$ & & & \\
\hline $5.000181 \mathrm{E}-03$ & $-7.379268 \mathrm{E}-04$ & & & & $3 / 17 / 9910: 01: 43$ & & & \\
\hline$-5.000672 E-03$ & $7.340986 \mathrm{E}-04$ & & & & $3 / 17 / 9910: 01: 46$ & & & \\
\hline$-5.000672 \mathrm{E}-03$ & 7.340947E-04 & & & & $3 / 17 / 9910: 01: 49$ & & & \\
\hline $5.000190 \mathrm{E}-03$ & $-7.379316 \mathrm{E}-04$ & & & & $3 / 17 / 99$ 10:01:51 & & & \\
\hline $5.000191 \mathrm{E}-03$ & $-7.379365 E-04$ & & & & $3 / 17 / 99$ 10:01:54 & & & \\
\hline$-5.000680 \mathrm{E}-03$ & 7.340734E-04 & & & & $3 / 17 / 99$ 10:01:57 & & & \\
\hline$-5.000676 \mathrm{E}-03$ & $7.340898 \mathrm{E}-04$ & & & & $3 / 17 / 9910: 02: 00$ & & & \\
\hline $5.000185 \mathrm{E}-03$ & $-7.379311 E-04$ & & & & $3 / 17 / 9910: 02: 03$ & & & \\
\hline 5.000187E-03 & $-7.379393 \mathrm{E}-04$ & & & & $3 / 17 / 99$ 10:02:06 & & & \\
\hline$-5.000678 \mathrm{E}-03$ & $7.340902 \mathrm{E}-04$ & & & & $3 / 17 / 99$ 10:02:08 & & & \\
\hline$-5.000686 \mathrm{E}-03$ & 7.340893E-04 & & & & $3 / 17 / 9910: 02: 11$ & & & \\
\hline $5.000178 \mathrm{E}-03$ & $-7.379358 \mathrm{E}-04$ & & & & $3 / 17 / 9910: 02: 14$ & & & \\
\hline $5.000179 \mathrm{E}-03$ & $-7.379320 \mathrm{E}-04$ & & & & $3 / 17 / 99$ 10:02:17 & & & \\
\hline$-5.000689 \mathrm{E}-03$ & 7.340918E-04 & & & & $3 / 17 / 9910: 02: 20$ & & & \\
\hline$-5.000686 \mathrm{E}-03$ & 7.340833E-04 & & & & $3 / 17 / 99$ 10:02:23 & & & \\
\hline 5.000180E-03 & $-7.379452 E-04$ & & & & $3 / 17 / 9910: 02: 25$ & & & \\
\hline 5.000183E-03 & $-7.379310 \mathrm{E}-04$ & & & & $3 / 17 / 9910: 02: 28$ & & & \\
\hline$-5.000686 \mathrm{E}-03$ & 7.340899E-04 & & & & $3 / 17 / 9910: 02: 31$ & & & \\
\hline$-5.000686 \mathrm{E}-03$ & 7.340778E-04 & & & & $3 / 17 / 9910: 02: 34$ & & & \\
\hline $5.000181 \mathrm{E}-03$ & $-7.379339 E-04$ & & & & $3 / 17 / 9910: 02: 37$ & & & \\
\hline $5.000181 \mathrm{E}-03$ & $-7.379451 \mathrm{E}-04$ & & & & $3 / 17 / 99 \quad 10: 02: 39$ & & & \\
\hline$-5.000693 \mathrm{E}-03$ & $7.340911 \mathrm{E}-04$ & & & & $3 / 17 / 9910: 02: 42$ & & & \\
\hline$-5.000690 \mathrm{E}-03$ & 7.340863E-04 & & & & $3 / 17 / 9910: 02: 45$ & & & \\
\hline $5.000185 \mathrm{E}-03$ & $-7.379294 \mathrm{E}-04$ & & & & $3 / 17 / 9910: 02: 48$ & & & \\
\hline $5.000183 E-03$ & $-7.379245 E-04$ & & & & $3 / 17 / 9910: 02: 51$ & & & \\
\hline$-5.000686 \mathrm{E}-03$ & $7.340890 \mathrm{E}-04$ & & & & $3 / 17 / 9910: 02: 54$ & & & \\
\hline$-5.000683 \mathrm{E}-03$ & 7.340957E-04 & & & & $3 / 17 / 99$ 10:02:56 & & & \\
\hline $5.000178 \mathrm{E}-03$ & $-7.379267 \mathrm{E}-04$ & & & & $3 / 17 / 9910: 02: 59$ & & & \\
\hline $5.000432 \mathrm{E}-03$ & 7.360106E-04 & 0.1471894 & $9.085980 \mathrm{E}-07$ & 3.680373E-06 & $3 / 17 / 99$ 10:03:03 & 4974.351282 & 4.727875166 & \\
\hline & & & & & & & & \\
\hline
\end{tabular}


Copper1.xis

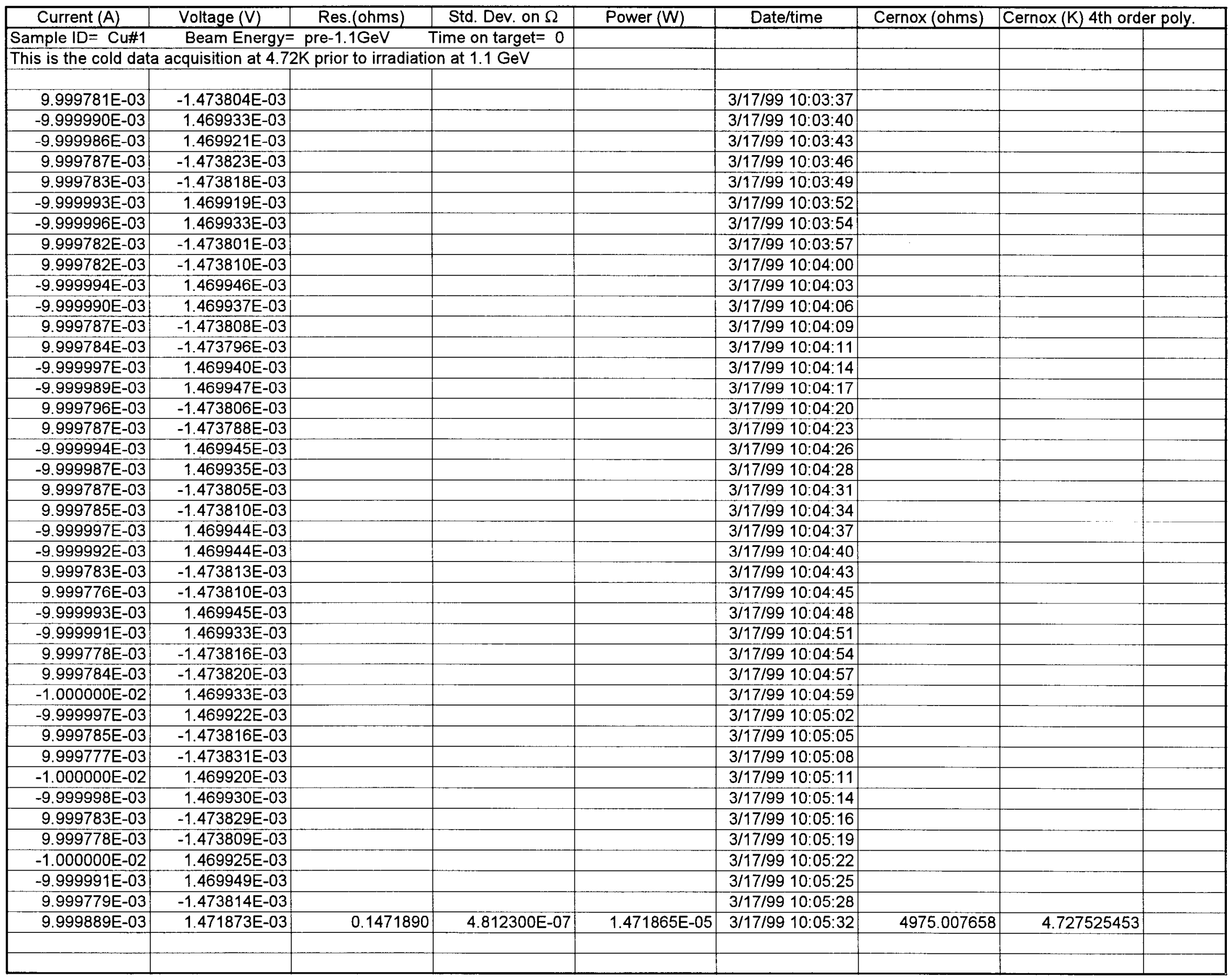


Copper1.xls

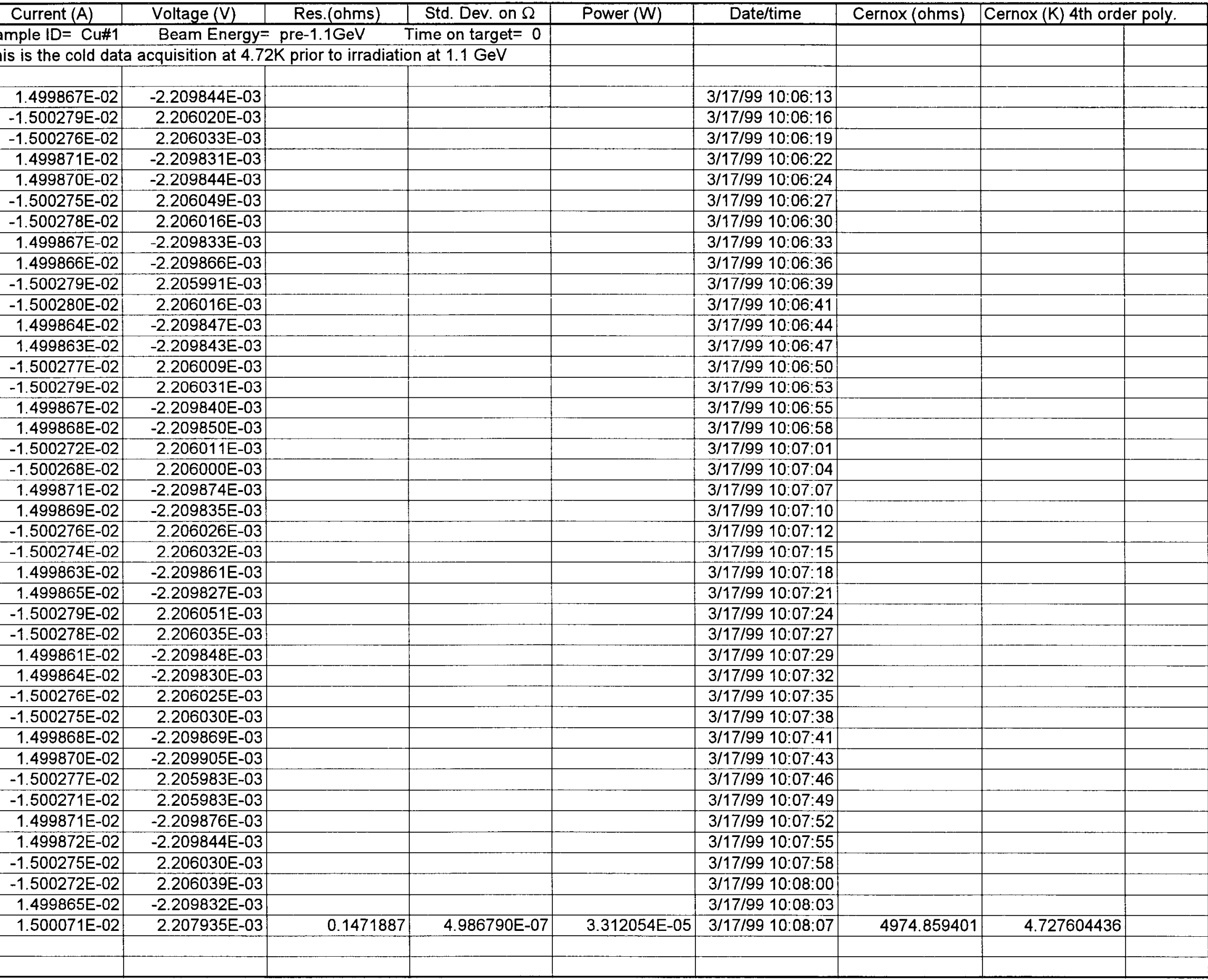


Copper1.xIs

\begin{tabular}{|c|c|c|c|c|c|c|c|c|}
\hline Current $(\mathrm{A})$ & Voltage (V) & Res.(ohms) & Std. Dev. on $\Omega$ & Power (W) & Date/time & Cernox (ohms) & \multicolumn{2}{|c|}{ Cernox (K) 4th order poly. } \\
\hline Sample ID= Cu\#1 & \multicolumn{2}{|c|}{ Beam Energy $=$ pre-1.1GeV } & Time on target $=0$ & & & & & \\
\hline \multicolumn{9}{|c|}{ This is the cold data acquisition at $4.72 \mathrm{~K}$ prior to irradiation at $1.1 \mathrm{GeV}$} \\
\hline & & & & & & & & \\
\hline $9.999767 \mathrm{E}-03$ & $-1.473958 \mathrm{E}-03$ & & & & $3 / 17 / 9910: 24: 28$ & & & \\
\hline$-1.000001 \mathrm{E}-02$ & $1.469806 \mathrm{E}-03$ & & & & $3 / 17 / 9910: 24: 31$ & & & \\
\hline$-1.000000 \mathrm{E}-02$ & 1.469807E-03 & & & & $3 / 17 / 9910: 24: 34$ & & & \\
\hline $9.999774 \mathrm{E}-03$ & $-1.473951 E-03$ & & & & $3 / 17 / 9910: 24: 36$ & & & \\
\hline $9.999773 \mathrm{E}-03$ & $-1.473934 \mathrm{E}-03$ & & & & $3 / 17 / 99 \quad 10: 24: 39$ & & & \\
\hline$-1.000000 \mathrm{E}-02$ & $1.469823 \mathrm{E}-03$ & & & & $3 / 17 / 99 \quad 10: 24: 42$ & & & \\
\hline$-9.999996 \mathrm{E}-03$ & $1.469822 \mathrm{E}-03$ & & & & $3 / 17 / 9910: 24: 45$ & & & \\
\hline $9.999786 \mathrm{E}-03$ & $-1.473938 E-03$ & & & & $3 / 17 / 99 \quad 10: 24: 48$ & & & \\
\hline $9.999776 \mathrm{E}-03$ & $-1.473931 \mathrm{E}-03$ & & & & $3 / 17 / 9910: 24: 51$ & & & \\
\hline$-1.000000 \mathrm{E}-02$ & $1469849 \mathrm{E}-03$ & & & & $3 / 17 / 9910: 24: 53$ & & & \\
\hline$-9.999998 E-03$ & $1.469854 \mathrm{E}-03$ & & & & $3 / 17 / 9910: 24: 56$ & & & \\
\hline $9.999775 \mathrm{E}-03$ & $-1.473922 E-03$ & & & & $3 / 17 / 9910: 24: 59$ & & & \\
\hline $9.999771 \mathrm{E}-03$ & $-1.473923 E-03$ & & & & $3 / 17 / 9910: 25: 02$ & & & \\
\hline$-1.000000 \mathrm{E}-02$ & $1.469852 \mathrm{E}-03$ & & & & $3 / 17 / 99$ 10:25:05 & & & \\
\hline$-1.000001 E-02$ & 1.469843E-03 & & & & $3 / 17 / 99$ 10:25:08 & & & \\
\hline $9.999771 \mathrm{E}-03$ & $-1.473922 \mathrm{E}-03$ & & & & $3 / 17 / 99$ 10:25:10 & & & \\
\hline 9.999769E-03 & $-1.473913 \mathrm{E}-03$ & & & & $3 / 17 / 99 \quad 10: 25: 13$ & & & \\
\hline$-1.000000 \mathrm{E}-02$ & 1.469853E-03 & & & & $3 / 17 / 9910: 25: 16$ & & & \\
\hline$-1.000000 \mathrm{E}-02$ & $1.469848 \mathrm{E}-03$ & & & & $3 / 17 / 99$ 10:25:19 & & & \\
\hline 9.999777E-03 & $-1.473912 \mathrm{E}-03$ & & & & $3 / 17 / 99 \quad 10: 25: 22$ & & & \\
\hline $9.999773 \mathrm{E}-03$ & $-1.473914 \mathrm{E}-03$ & & & & $3 / 17 / 99 \quad 10: 25: 24$ & & & \\
\hline$-1.000001 E-02$ & 1.469847E-03 & & & & $3 / 17 / 9910: 25: 27$ & & & \\
\hline$-1.000001 E-02$ & 1.469839E-03 & & & & $3 / 17 / 9910: 25: 30$ & & & \\
\hline $9.999775 \mathrm{E}-03$ & $-1.473910 \mathrm{E}-03$ & & & & $3 / 17 / 9910: 25: 33$ & & & \\
\hline $9.999769 \mathrm{E}-03$ & $-1.473919 \mathrm{E}-03$ & & & & $3 / 17 / 99 \quad 10: 25: 36$ & & & \\
\hline$-1.000002 E-02$ & $1.469846 \mathrm{E}-03$ & & & & $3 / 17 / 99$ 10:25:39 & & & \\
\hline$-1.000001 \mathrm{E}-02$ & 1.469852E-03 & & & & $3 / 17 / 99 \quad 10: 25: 41$ & & & \\
\hline $9.999775 \mathrm{E}-03$ & $-1.473923 E-03$ & & & & $3 / 17 / 9910: 25: 44$ & & & \\
\hline $9.999774 \mathrm{E}-03$ & $-1.473908 \mathrm{E}-03$ & & & & $3 / 17 / 99$ 10:25:47 & & & \\
\hline$-1.000001 E-02$ & $1.469840 \mathrm{E}-03$ & & & & $3 / 17 / 9910: 25: 50$ & & & \\
\hline$-1.000001 \mathrm{E}-02$ & $1.469848 \mathrm{E}-03$ & & & & $3 / 17 / 99 \quad 10: 25: 53$ & & & \\
\hline $9.999780 \mathrm{E}-03$ & $-1.473917 \mathrm{E}-03$ & & & & 3/17/99 10:25:56 & & & \\
\hline $9.999769 \mathrm{E}-03$ & $-1.473911 \mathrm{E}-03$ & & & & $3 / 17 / 99 \quad 10: 25: 58$ & & & \\
\hline$-1.000001 E-02$ & $1.469862 \mathrm{E}-03$ & & & & $3 / 17 / 99$ 10:26:01 & & & \\
\hline$-1.000001 E-02$ & $1.469866 \mathrm{E}-03$ & & & & $3 / 17 / 99$ 10:26:04 & & & \\
\hline $9.999775 \mathrm{E}-03$ & $-1.473911 E-03$ & & & & $3 / 17 / 9910: 26: 07$ & & & \\
\hline $9.999775 \mathrm{E}-03$ & $-1.473918 \mathrm{E}-03$ & & & & $3 / 17 / 99$ 10:26:10 & & & \\
\hline$-1.000001 E-02$ & $1.469844 \mathrm{E}-03$ & & & & $3 / 17 / 99 \quad 10: 26: 13$ & & & \\
\hline$-1.000001 E-02$ & $1.469840 \mathrm{E}-03$ & & & & $3 / 17 / 99$ 10:26:15 & & & \\
\hline $9.999779 E-03$ & $-1.473912 E-03$ & & & & $3 / 17 / 99$ 10:26:18 & & & \\
\hline $9.999890 \mathrm{E}-03$ & $1.471882 E-03$ & 0.1471898 & $4.724915 \mathrm{E}-07$ & $1.471860 \mathrm{E}-05$ & $3 / 17 / 99 \quad 10: 26: 22$ & 4976.104152 & 4.726941434 & \\
\hline & & & & & & & & \\
\hline & & & & & & & & \\
\hline
\end{tabular}


Copper $1 . x / s$

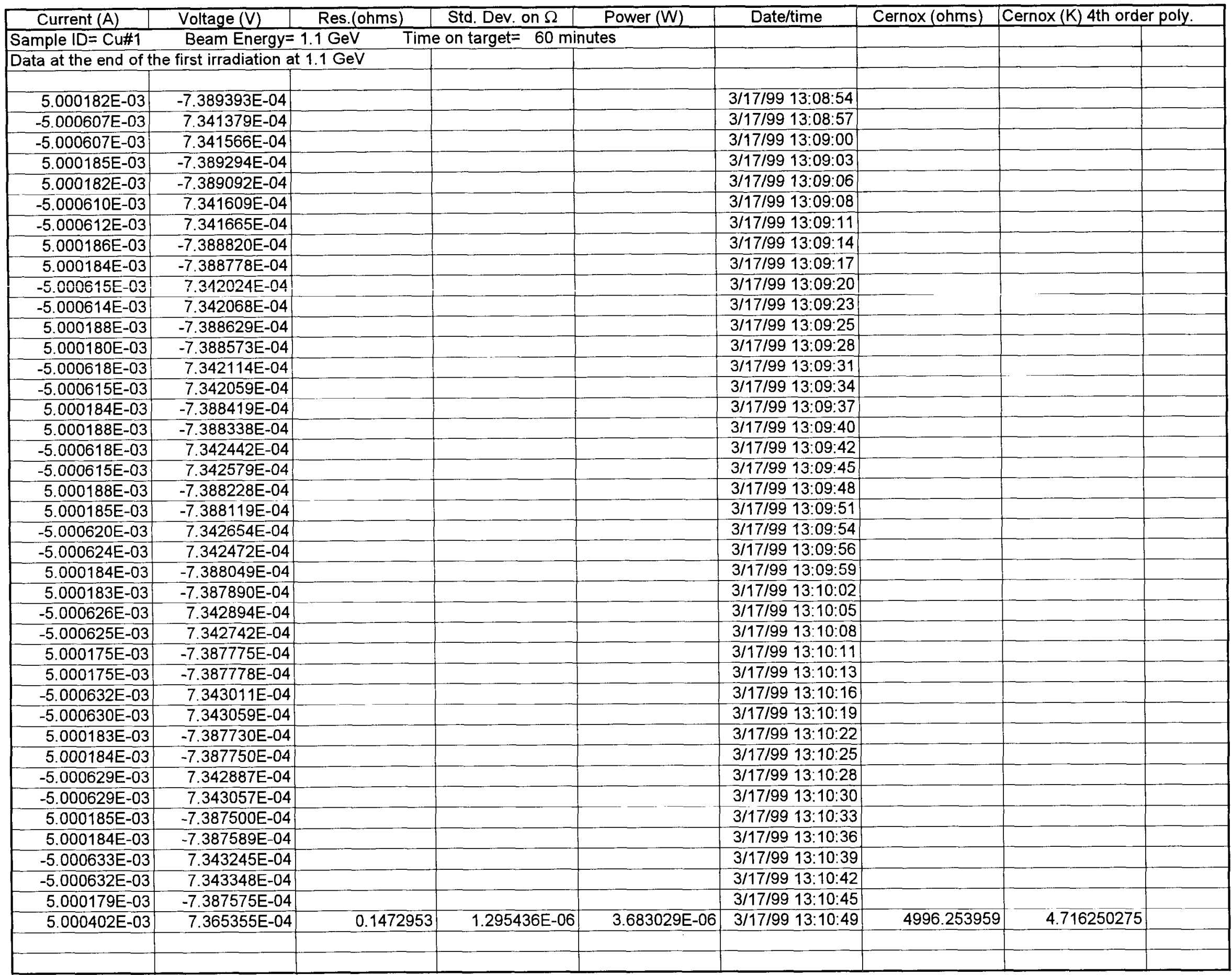


Copper1.xIs

\begin{tabular}{|c|c|c|c|c|c|c|c|c|}
\hline Current $(\mathrm{A})$ & Voltage (V) & Res.(ohms) & Std. Dev. on $\Omega$ & Power (W) & Date/time & Cernox (ohms) & \multicolumn{2}{|c|}{ Cernox (K) 4th order poly. } \\
\hline Sample ID= Cu\#1 & \multicolumn{2}{|c|}{ Beam Energy $=1.1 \mathrm{GeV}$} & \multicolumn{2}{|c|}{ Time on target $=60$ minutes } & & & & \\
\hline \multicolumn{9}{|c|}{ Data at the end of the first irradiation at $1.1 \mathrm{GeV}$} \\
\hline & & & & & & & & \\
\hline $9.999803 \mathrm{E}-03$ & $-1.475119 \mathrm{E}-03$ & & & & $3 / 17 / 9913: 11: 34$ & & & \\
\hline$-9.999961 \mathrm{E}-03$ & $1.470745 \mathrm{E}-03$ & & & & $3 / 17 / 9913: 11: 37$ & & & \\
\hline$-9.999958 \mathrm{E}-03$ & $1.470751 \mathrm{E}-03$ & & & & $3 / 17 / 9913: 11: 40$ & & & \\
\hline $9.999808 \mathrm{E}-03$ & $-1.475131 \mathrm{E}-03$ & & & & $3 / 17 / 99 \quad 13: 11: 43$ & & & \\
\hline $9.999802 \mathrm{E}-03$ & $-1.475122 \mathrm{E}-03$ & & & & $3 / 17 / 9913: 11: 45$ & & & \\
\hline$-9.999963 \mathrm{E}-03$ & 1.470751E-03 & & & & $3 / 17 / 99 \quad 13: 11: 48$ & & & \\
\hline$-9.999962 E-03$ & $1.470754 \mathrm{E}-03$ & & & & $3 / 17 / 9913: 11: 51$ & & & \\
\hline 9.999810E-03 & $-1.475104 \mathrm{E}-03$ & & & & $3 / 17 / 9913: 11: 54$ & & & \\
\hline $9.999803 \mathrm{E}-03$ & $-1.475107 \mathrm{E}-03$ & & & & $3 / 17 / 99$ 13:11:57 & & & \\
\hline$-3.333970 E-03$ & $1.470763 \mathrm{E}-03$ & & & & $3 / 17 / 9913: 12: 00$ & & & \\
\hline$-9.999969 E-03$ & $1.470768 \mathrm{E}-03$ & & & & $3 / 17 / 99 \quad 13: 12: 02$ & & & \\
\hline $9.999804 \mathrm{E}-03$ & $-1.475103 \mathrm{E}-03$ & & & & $3 / 17 / 9913: 12: 05$ & & & \\
\hline $9.999799 \mathrm{E}-03$ & $-1.475095 E-03$ & & & & $3 / 17 / 9913: 12: 08$ & & & \\
\hline$-9.999968 \mathrm{E}-03$ & $1.470768 \mathrm{E}-03$ & & & & $3 / 17 / 9913: 12: 11$ & & & \\
\hline$-9.999965 E-03$ & $1.470770 \mathrm{E}-03$ & & & & $3 / 17 / 9913: 12: 14$ & & & \\
\hline $9.999803 E-03$ & $-1.475104 E-03$ & & & & $3 / 17 / 9913: 12: 17$ & & & \\
\hline $9.999800 \mathrm{E}-03$ & $-1.475095 \mathrm{E}-03$ & & & & $3 / 17 / 9913: 12: 19$ & & & \\
\hline$-9.999972 E-03$ & $1.470778 \mathrm{E}-03$ & & & & $3 / 17 / 99 \quad 13: 12: 22$ & & & \\
\hline$-9.999971 \mathrm{E}-03$ & $1.470769 \mathrm{E}-03$ & & & & $3 / 17 / 9913: 12: 25$ & & & \\
\hline $9.999803 \mathrm{E}-03$ & $-1.475108 E-03$ & & & & $3 / 17 / 9913: 12: 28$ & & & \\
\hline $9.999800 \mathrm{E}-03$ & $-1.475088 \mathrm{E}-03$ & & & & $3 / 17 / 9913: 12: 31$ & & & \\
\hline$-9.999979 E-03$ & $1.470785 \mathrm{E}-03$ & & & & $3 / 17 / 99$ 13:12:34 & & & \\
\hline$-9.999972 E-03$ & $1.470781 \mathrm{E}-03$ & & & & $3 / 17 / 9913: 12: 36$ & & & \\
\hline $9.999798 \mathrm{E}-03$ & $-1.475072 \mathrm{E}-03$ & & & & $3 / 17 / 9913: 12: 39$ & & & \\
\hline $9.999793 \mathrm{E}-03$ & $-1.475089 \mathrm{E}-03$ & & & & $3 / 17 / 9913: 12: 42$ & & & \\
\hline$-9.999983 E-0 \overline{3}$ & $1.470794 \mathrm{E}-03$ & & & & $3 / 17 / 9913: 12: 45$ & & & \\
\hline$-9.999980 \mathrm{E}-03$ & $1.470794 \mathrm{E}-03$ & & & & $3 / 17 / 99$ 13:12:48 & & & \\
\hline $9.999793 \mathrm{E}-03$ & $-1.475071 \mathrm{E}-03$ & & & & $3 / 17 / 99$ 13:12:51 & & & \\
\hline $9.999792 E-03$ & $-1.475075 E-03$ & & & & $3 / 17 / 99 \quad 13: 12: 53$ & & & \\
\hline$-9.999985 \mathrm{E}-03$ & $1.470810 \mathrm{E}-03$ & & & & $3 / 17 / 99$ 13:12:56 & & & \\
\hline -9.999977E-03 & $1 . \overline{47} 0788 \mathrm{E}-03$ & & & & $3 / 17 / 9913: 12: 59$ & & & \\
\hline $9.999796 \mathrm{E}-03$ & $-1.475087 \mathrm{E}-03$ & & & & 3/17/99 13:13:02 & & & \\
\hline $9.999793 E-03$ & $-1.475080 \mathrm{E}-03$ & & & & 3/17/99 13:13:05 & & & \\
\hline$-9.999983 \mathrm{E}-03$ & $1.470793 \mathrm{E}-03$ & & & & $3 / 17 / 9913: 13: 07$ & & & \\
\hline$-9.999980 \mathrm{E}-03$ & 1.470787E-03 & & & & $3 / 17 / 99 \quad 13: 13: 10$ & & & \\
\hline $9.999793 \mathrm{E}-03$ & $-1.475082 \mathrm{E}-03$ & & & - & 3/17/99 13:13:13 & & & \\
\hline $9.999790 \mathrm{E}-03$ & $-1.475066 \mathrm{E}-03$ & & & & $3 / 17 / 9913: 13: 16$ & & & \\
\hline$-9.999986 E-03$ & $1.470784 \mathrm{E}-03$ & & & & 3/17/99 13:13:19 & & & \\
\hline$-9.999983 \mathrm{E}-03$ & $1.470802 \mathrm{E}-03$ & & & & $3 / 17 / 9913: 13: 22$ & & & \\
\hline $9.999789 \mathrm{E}-03$ & $-1.475080 \mathrm{E}-03$ & & & & $3 / 17 / 9913: 13: 24$ & & & \\
\hline $9.999886 \mathrm{E}-03$ & 1.472935E-03 & 0.1472952 & $4.562880 \mathrm{E}-07$ & 1.472924E-05 & $3 / 17 / 9913: 13: 29$ & 4992.582791 & 4.718192347 & \\
\hline & & & & & & & & \\
\hline
\end{tabular}


Copper1.x/s

\begin{tabular}{|c|c|c|c|c|c|c|c|c|}
\hline Current (A) & Voltage (V) & Res.(ohms) & Std. Dev. on $\Omega$ & Power (W) & Date/time & Cernox (ohms) & \multicolumn{2}{|c|}{ Cernox $(\mathrm{K})$ 4th order poly. } \\
\hline Sample ID = Cu\#1 & \multicolumn{2}{|c|}{ Beam Energy $=1.1 \mathrm{GeV}$} & \multicolumn{2}{|c|}{ Time on target $=60$ minutes } & & & & \\
\hline \multicolumn{9}{|c|}{ Data at the end of the first irradiation at $1.1 \mathrm{GeV}$} \\
\hline & & & & & & & & \\
\hline 1.499852E-02 & $-2.211611 \mathrm{E}-03$ & & & & $3 / 17 / 9913: 13: 56$ & & & \\
\hline$-1.500233 \mathrm{E}-02$ & $2.207332 \mathrm{E}-03$ & & & & $3 / 17 / 9913: 13: 58$ & & & \\
\hline$-1.500231 \mathrm{E}-02$ & $2.207332 \mathrm{E}-03$ & & & & $3 / 17 / 9913: 14: 01$ & & & \\
\hline $1.499858 \mathrm{E}-02$ & $-2.211631 \mathrm{E}-03$ & & & & $3 / 17 / 9913: 14: 04$ & & & \\
\hline 1.499861E-02 & $-2.211645 \mathrm{E}-03$ & & & & $3 / 17 / 9913: 14: 07$ & & & \\
\hline$-1.500237 \mathrm{E}-02$ & $2.207340 \mathrm{E}-03$ & & & & $3 / 17 / 9913: 14: 10$ & & & \\
\hline$-1.500234 \mathrm{E}-02$ & $2.207327 \mathrm{E}-03$ & & & & $3 / 17 / 9913: 14: 13$ & & & \\
\hline $1.499861 \mathrm{E}-02$ & $-2.211616 \mathrm{E}-03$ & & & & $3 / 17 / 9913: 14: 15$ & & & \\
\hline 1.499863E-02 & $-2.211653 \mathrm{E}-03$ & & & & $3 / 17 / 9913: 14: 18$ & & & \\
\hline$-1.500230 E-02$ & $2.207304 E-0.3$ & & & & $3 / 17 / 9913: 14: 21$ & & & \\
\hline$-1.500232 E-02$ & $2.207305 \mathrm{E}-03$ & & & & $3 / 17 / 9913: 14: 24$ & & & \\
\hline $1.499866 \mathrm{E}-02$ & $-2.211666 \mathrm{E}-03$ & & & & $3 / 17 / 9913: 14: 27$ & & & \\
\hline $1.499865 \mathrm{E}-02$ & $-2.211654 E-03$ & & & & $3 / 17 / 9913: 14: 30$ & & & \\
\hline$-1.500232 \mathrm{E}-02$ & $2.207310 \mathrm{E}-03$ & & & & $3 / 17 / 9913: 14: 32$ & & & \\
\hline$-1.500233 \mathrm{E}-02$ & $2.207302 \mathrm{E}-03$ & & & & $3 / 17 / 9913: 14: 35$ & & & \\
\hline $1.499865 \mathrm{E}-02$ & $-2.211676 \mathrm{E}-03$ & & & & $3 / 17 / 9913: 14: 38$ & & & \\
\hline $1.499866 \mathrm{E}-02$ & $-2.211652 \mathrm{E}-03$ & & & & $3 / 17 / 99$ 13:14:41 & & & \\
\hline$-1.500233 E-02$ & $2.207318 \mathrm{E}-03$ & & & & $3 / 17 / 9913: 14: 44$ & & & \\
\hline$-1.500233 E-02$ & $2.207286 \mathrm{E}-03$ & & & & $3 / 17 / 9913: 14: 46$ & & & \\
\hline $1.499866 \mathrm{E}-02$ & $-2.211666 \mathrm{E}-03$ & & & & $3 / 17 / 9913: 14: 49$ & & & \\
\hline 1.499869E-02 & $-2.211667 \mathrm{E}-03$ & & & & $3 / 17 / 9913: 14: 52$ & & & \\
\hline$-1.500234 \mathrm{E}-02$ & $2.207318 \mathrm{E}-03$ & & & & $3 / 17 / 9913: 14: 55$ & & & \\
\hline$-1.500232 E-02$ & $2.207336 \mathrm{E}-03$ & & & & $3 / 17 / 9913: 14: 58$ & & & \\
\hline 1.499863E-02 & $-2.211669 \mathrm{E}-03$ & & & & $3 / 17 / 9913: 15: 01$ & & & \\
\hline $1.499866 \mathrm{E}-02$ & $-2.211648 \mathrm{E}-03$ & & & & $3 / 17 / 9913: 15: 03$ & & & \\
\hline$-1.500242 E-02$ & $2.207330 \mathrm{E}-03$ & & & & $3 / 17 / 9913: 15: 06$ & & & \\
\hline$-1.500233 E-02$ & $2.207302 \mathrm{E}-03$ & & & & $3 / 17 / 9913: 15: 09$ & & & \\
\hline $1.499862 \mathrm{E}-02$ & $-2.211629 E-03$ & & & & $3 / 17 / 9913: 15: 12$ & & & \\
\hline $1.499860 \mathrm{E}-02$ & $-2.211659 E-03$ & & & & $3 / 17 / 9913: 15: 15$ & & & \\
\hline$-1.500231 E-02$ & $2.207315 \mathrm{E}-03$ & & & & $3 / 17 / 9913: 15: 18$ & & & \\
\hline$-1.500235 \mathrm{E}-02$ & $2.207315 \mathrm{E}-03$ & & & & $3 / 17 / 9913: 15: 20$ & & & \\
\hline 1.499865E-02 & $-2.211666 \mathrm{E}-03$ & & & & $3 / 17 / 99$ 13:15:23 & & & \\
\hline 1.499872E-02 & $-2.211672 E-03$ & & & & $3 / 17 / 9913: 15: 26$ & & & \\
\hline$-1.500234 \mathrm{E}-02$ & $2.207310 \mathrm{E}-03$ & & & & $3 / 17 / 9913: 15: 29$ & & & \\
\hline$-1.500235 E-02$ & $2.207302 \mathrm{E}-03$ & & & & $3 / 17 / 99$ 13:15:32 & & & \\
\hline $1.499866 \mathrm{E}-02$ & $-2.211642 E-03$ & & & & $3 / 17 / 99$ 13:15:35 & & & \\
\hline $1.499872 \mathrm{E}-02$ & $-2.211637 \mathrm{E}-03$ & & & & $3 / 17 / 9913: 15: 37$ & & & \\
\hline$-1.500232 \mathrm{E}-02$ & $2.207338 \mathrm{E}-03$ & & & & $3 / 17 / 9913: 15: 40$ & & & \\
\hline$-1.500238 E-02$ & $2.207332 \mathrm{E}-03$ & & & & $3 / 17 / 9913: 15: 43$ & & & \\
\hline $1.499869 \mathrm{E}-02$ & $-2.211657 \mathrm{E}-03$ & & & & $3 / 17 / 9913: 15: 46$ & & & \\
\hline $1.500049 \mathrm{E}-02$ & $2.209484 \mathrm{E}-03$ & 0.1472941 & $5.504603 \mathrm{E}-07$ & $3.314360 \mathrm{E}-05$ & $3 / 17 / 9913: 15: 50$ & 4991.524407 & 4.718752717 & \\
\hline & & & & & & & & \\
\hline & & & & & & & & \\
\hline
\end{tabular}


Copper1.xls

\begin{tabular}{|c|c|c|c|c|c|c|c|c|}
\hline Current $(A)$ & Voltage (V) & Res.(ohms) & Std. Dev. on $\Omega$ & Power (W) & Date/time & Cernox (ohms) & \multicolumn{2}{|c|}{ Cernox $(K)$ 4th order poly. } \\
\hline Sample ID= Cu\#1 & \multicolumn{2}{|c|}{ Beam Energy $=1.1 \mathrm{Gev}$} & on target $=135 \mathrm{~min}$ & tes & & & & \\
\hline \multicolumn{9}{|c|}{ End of second and last irradiation at $1.1 \mathrm{GeV}$} \\
\hline & & & & & & & & \\
\hline $5.000189 E-03$ & $-7.403216 E-04$ & & & & $3 / 17 / 9915: 29: 11$ & & & \\
\hline$-5.000414 \mathrm{E}-03$ & $7.358276 \mathrm{E}-04$ & & & & $3 / 17 / 9915: 29: 14$ & & & \\
\hline$-5.000415 E-03$ & 7.358165E-04 & & & & $3 / 17 / 99 \quad 15: 29: 16$ & & & \\
\hline $5.000189 \mathrm{E}-03$ & $-7.403141 \mathrm{E}-04$ & & & & $3 / 17 / 99$ 15:29:19 & & & \\
\hline 5.000191E-03 & $-7.403152 \mathrm{E}-04$ & & & & $3 / 17 / 99 \quad 15: 29: 22$ & & & \\
\hline$-5.000419 \mathrm{E}-03$ & $7.358298 \mathrm{E}-04$ & & & & 3/17/99 15:29:25 & & & \\
\hline$-5.000417 \mathrm{E}-03$ & $7.358330 \mathrm{E}-04$ & & & & $3 / 17 / 99$ 15:29:28 & & & \\
\hline $5.000189 \mathrm{E}-03$ & $-7.403159 \mathrm{E}-04$ & & & & $3 / 17 / 9915: 29: 31$ & & & \\
\hline $5.000188 \mathrm{E}-03$ & $-7.403140 \mathrm{E}-04$ & & & & 3/17/99 15:29:33 & & & \\
\hline$-5.000420 \mathrm{E}-03$ & $7.358322 \mathrm{E}-04$ & & & & 3/17/99 15:29:36 & & & \\
\hline$-5.000420 \mathrm{E}-03$ & $7.358328 \mathrm{E}-04$ & & & & $3 / 17 / 9915: 29: 39$ & & & \\
\hline $5.000190 \mathrm{E}-03$ & $-7.403090 \mathrm{E}-04$ & & & & $3 / 17 / 9915: 29: 42$ & & & \\
\hline $5.000188 \mathrm{E}-03$ & $-7.403064 \mathrm{E}-04$ & & & & $3 / 17 / 9915: 29: 45$ & & & \\
\hline$-5.000423 E-03$ & $7.358489 \mathrm{E}-04$ & & & & $3 / 17 / 9915: 29: 48$ & & & \\
\hline$-5.000421 \mathrm{E}-03$ & $7.358374 \mathrm{E}-04$ & & & & 3/17/99 15:29:50 & & & \\
\hline $5.0 \overline{001} 93 \mathrm{E}-03$ & $-7.403104 E-04$ & & & & $3 / 17 / 99 \quad 15: 29: 53$ & & & \\
\hline $5.000193 \mathrm{E}-03$ & $-7.403051 \mathrm{E}-04$ & & & & $3 / 17 / 9915: 29: 56$ & & & \\
\hline$-5.000425 \mathrm{E}-03$ & $7.358387 E-04$ & & & & 3/17/99 15:29:59 & & & \\
\hline$-5.000423 E-03$ & $7.358438 \mathrm{E}-04$ & & & & 3/17/99 15:30:02 & & & \\
\hline $5.000192 \mathrm{E}-03$ & $-7.403011 E-04$ & & & & 3/17/99 15:30:05 & & & \\
\hline $5.000192 \mathrm{E}-03$ & $-7.403034 \mathrm{E}-04$ & & & & $3 / 17 / 9915: 30: 07$ & & & \\
\hline$-5.000428 \mathrm{E}-03$ & $7.358368 \mathrm{E}-04$ & & & & $3 / 17 / 99$ 15:30:10 & & & \\
\hline$-5.000429 \mathrm{E}-03$ & $7.358408 \mathrm{E}-04$ & & & & 3/17/99 15:30:13 & & & \\
\hline $5.000194 \mathrm{E}-03$ & $-7.403041 \mathrm{E}-04$ & & & & 3/17/99 15:30:16 & & & \\
\hline $5.000190 \mathrm{E}-03$ & $-7.403010 \mathrm{E}-04$ & & & & $3 / 17 / 99$ 15:30:19 & & & \\
\hline$-5.000434 \mathrm{E}-03$ & $7.358448 \mathrm{E}-04$ & & & & $3 / 17 / 99$ 15:30:21 & & & \\
\hline$-5.000429 \mathrm{E}-03$ & $7.358437 \mathrm{E}-04$ & & & & $3 / 17 / 99$ 15:30:24 & & & \\
\hline $5.000193 \mathrm{E}-03$ & $-7.403037 \mathrm{E}-04$ & & & & $3 / 17 / 9915: 30: 27$ & & & \\
\hline $5.000192 \mathrm{E}-03$ & $-7.402992 E-04$ & & & & 3/17/99 15:30:30 & & & \\
\hline$-5.000432 \mathrm{E}-03$ & $7.358401 \mathrm{E}-04$ & & & & $3 / 17 / 99 \quad 15: 30: 33$ & & & \\
\hline$-5.000435 \mathrm{E}-03$ & 7.358393E-04 & & & & $3 / 17 / 99$ 15:30:36 & & & \\
\hline $5.000189 \mathrm{E}-03$ & $-7.403132 \mathrm{E}-04$ & & & & $3 / 17 / 99 \quad 15: 30: 38$ & & & \\
\hline $5.000191 \mathrm{E}-03$ & $-7.402983 E-04$ & & & & $3 / 17 / 9915: 30: 41$ & & & \\
\hline$-5.000436 \mathrm{E}-03$ & $7.358430 \mathrm{E}-04$ & & & & $3 / 17 / 9915: 30: 44$ & & & \\
\hline$-5.000436 \mathrm{E}-03$ & $7.358467 \mathrm{E}-04$ & \multirow[b]{2}{*}{ - } & & & $3 / 17 / 9915: 30: 47$ & & & \\
\hline $5.000192 \mathrm{E}-03$ & $-7.402984 \mathrm{E}-04$ & & & & $3 / 17 / 9915: 30: 50$ & & & \\
\hline $5.000190 \mathrm{E}-03$ & $-7.403003 E-04$ & & & & $3 / 17 / 99$ 15:30:53 & & & \\
\hline$-5.000440 \mathrm{E}-03$ & $7.358446 \mathrm{E}-04$ & & & & $3 / 17 / 99$ 15:30:55 & & & \\
\hline$-5.000440 \mathrm{E}-03$ & $7.358413 \mathrm{E}-04$ & & & & 3/17/99 15:30:58 & & & \\
\hline $5.000193 \mathrm{E}-03$ & $-7.403002 \mathrm{E}-04$ & & & & $3 / 17 / 99$ 15:31:01 & & & \\
\hline $5.000309 \mathrm{E}-03$ & $7.380724 \mathrm{E}-04$ & 0.1476054 & $5.292717 \mathrm{E}-07$ & $3.69058 \overline{7} E-06$ & $3 / 17 / 9915: 31: 05$ & 4977.762953 & 4.726058357 & \\
\hline & & & & & & & & \\
\hline & & & & & & & & \\
\hline
\end{tabular}


Copper1.xls

\begin{tabular}{|c|c|c|c|c|c|c|c|c|}
\hline Current (A) & Voltage (V) & Res.(ohms) & Std. Dev. on $\Omega$ & Power (W) & Date/time & Cernox (ohms) & \multicolumn{2}{|c|}{ Cernox $(K)$ 4th order poly. } \\
\hline Sample ID= Cu\#1 & \multicolumn{2}{|c|}{ Beam Energy $=1.1 \mathrm{Gev}$} & on target $=135 \mathrm{mir}$ & ites & & & & \\
\hline \multicolumn{9}{|c|}{ End of second and last irradiation at $1.1 \mathrm{GeV}$} \\
\hline & & & & & & & & \\
\hline $9.999865 \mathrm{E}-03$ & $-1.478250 \mathrm{E}-03$ & & & & 3/17/99 15:31:53 & & & \\
\hline$-9.999826 \mathrm{E}-03$ & $1.473786 \mathrm{E}-03$ & & & & $3 / 17 / 9915: 31: 56$ & & & \\
\hline$-9.999825 E-03$ & 1.473779E-03 & & & & $3 / 17 / 9915: 31: 59$ & & & \\
\hline $9.999869 \mathrm{E}-03$ & $-1.478253 \mathrm{E}-03$ & & & & 3/17/99 15:32:02 & & & \\
\hline 9.999864E-03 & $-1.478261 \mathrm{E}-03$ & & & & $3 / 17 / 9915: 32: 04$ & & & \\
\hline$-9.999831 \mathrm{E}-03$ & $1.473785 \mathrm{E}-03$ & & & & $3 / 17 / 9915: 32: 07$ & & & \\
\hline$-9.999828 E-03$ & $1.473792 \mathrm{E}-03$ & & & & $3 / 17 / 99$ 15:32:10 & & & \\
\hline $9.999868 \mathrm{E}-03$ & $-1.478266 \mathrm{E}-03$ & & & & $3 / 17 / 9915: 32: 13$ & & & \\
\hline 9.999864E-03 & $-1.478262 \mathrm{E}-03$ & & & & $3 / 17 / 9915: 32: 16$ & & & \\
\hline$-9.999836 \mathrm{E}-03$ & $1.473779 \mathrm{E}-03$ & & & & $3 / 17 / 99$ 15:32:19 & & & \\
\hline$-9.999833 \mathrm{E}-03$ & $1.473785 \mathrm{E}-03$ & & & & $3 / 17 / 99 \quad 15: 32: 21$ & & & \\
\hline $9.999863 \mathrm{E}-03$ & $-1.478259 \mathrm{E}-03$ & & & & $3 / 17 / 9915: 32: 24$ & & & \\
\hline 9.999857E-03 & $-1.478254 E-03$ & & & & 3/17/99 15:32:27 & & & \\
\hline$-9.999840 \mathrm{E}-03$ & $1.473799 \mathrm{E}-03$ & & & & $3 / 17 / 9915: 32: 30$ & & & \\
\hline$-9.999835 \mathrm{E}-03$ & $1.473792 \mathrm{E}-03$ & & & & $3 / 17 / 9915: 32: 33$ & & & \\
\hline $9.999859 \mathrm{E}-03$ & $-1.478251 E-03$ & & & & 3/17/99 15:32:36 & & & \\
\hline $9.999854 \mathrm{E}-03$ & $-1.478244 E-03$ & & & & $3 / 17 / 9915: 32: 38$ & & & \\
\hline$-9.999841 \mathrm{E}-03$ & $1.473782 \mathrm{E}-03$ & & & & $3 / 17 / 9915: 32: 41$ & & & \\
\hline$-9.999837 \mathrm{E}-03$ & 1.473783E-03 & & & & $3 / 17 / 9915: 32: 44$ & & & \\
\hline $9.999858 \mathrm{E}-03$ & $-1.478233 E-03$ & & & & $3 / 17 / 99$ 15:32:47 & & & \\
\hline $9.999855 \mathrm{E}-03$ & $-1.478241 \mathrm{E}-03$ & & & & $3 / 17 / 99 \quad 15: 32: 50$ & & & \\
\hline$-9.999841 E-03$ & $1.473789 \mathrm{E}-03$ & & & & $3 / 17 / 9915: 32: 53$ & & & \\
\hline$-9.999841 E-03$ & $1.473786 \mathrm{E}-03$ & & & & $3 / 17 / 9915: 32: 55$ & & & \\
\hline $9.999858 \mathrm{E}-03$ & $-1.478252 E-03$ & & & & $3 / 17 / 9915: 32: 58$ & & & \\
\hline $9.999855 \mathrm{E}-03$ & $-1.478243 \mathrm{E}-03$ & & & & 3/17/99 15:33:01 & & & \\
\hline$-9.999841 E-03$ & $1.473794 \mathrm{E}-03$ & & & & $3 / 17 / 99 \quad 15: 33: 04$ & & & \\
\hline$-9.999838 \mathrm{E}-03$ & $1.473785 E-03$ & & & & $3 / 17 / 9915: 33: 07$ & & & \\
\hline 9.999858E-03 & $-1.478241 E-03$ & & & & $3 / 17 / 9915: 33: 10$ & & & \\
\hline $9.999857 \mathrm{E}-03$ & $-1.478242 E-03$ & & & & $3 / 17 / 99$ 15:33:12 & & & \\
\hline$-9.999845 \mathrm{E}-03$ & $1.473783 \mathrm{E}-03$ & & & & $3 / 17 / 9915: 33: 15$ & & & \\
\hline$-9.99984 \overline{1 E-03}$ & 1.473790E-03 & & & & $3 / 17 / 99$ 15:33:18 & & & \\
\hline $9.999855 E-03$ & $-1.478234 E-03$ & & & & 3/17/99 15:33:21 & & & \\
\hline $9.999854 \mathrm{E}-03$ & $-1.478237 \mathrm{E}-03$ & & & & $3 / 17 / 99$ 15:33:24 & & & \\
\hline$-9.999837 \mathrm{E}-03$ & 1.473794E-03 & & & & 3/17/99 15:33:26 & & & \\
\hline$-9.999834 \mathrm{E}-03$ & 1.473791E-03 & \multirow[b]{2}{*}{ - } & & & $3 / 17 / 9915: 33: 29$ & & & \\
\hline 9.999854E-03 & $-1.47823 \overline{7 E-03}$ & & & & $3 / 17 / 9915: 33: 32$ & & & \\
\hline $9.999848 \mathrm{E}-03$ & $-1.478242 \mathrm{E}-03$ & & $\cdot$ & & $3 / 17 / 99$ 15:33:35 & & & \\
\hline$-9.999844 \mathrm{E}-03$ & $1.473792 \mathrm{E}-03$ & & & & $3 / 17 / 99 \quad 15: 33: 38$ & & & \\
\hline$-9.999841 E-03$ & $1.473802 \mathrm{E}-03$ & & & & $3 / 17 / 99$ 15:33:41 & & & \\
\hline $9.999851 \mathrm{E}-03$ & $-1.478243 \mathrm{E}-03$ & & & & $3 / 17 / 9915: 33: 43$ & & & \\
\hline $9.999848 \mathrm{E}-03$ & $1.476018 \mathrm{E}-03$ & 0.1476040 & $5.047303 \mathrm{E}-07$ & $1.475999 \mathrm{E}-0 \overline{5}$ & $3 / 17 / 9915: 33: 48$ & 4976.947725 & 4.726492284 & \\
\hline & & & & & & & & \\
\hline & & & & & & & & \\
\hline
\end{tabular}


Copper1.xls

\begin{tabular}{|c|c|c|c|c|c|c|c|c|}
\hline Current $(A)$ & Voltage $(V)$ & Res.(ohms) & Std. Dev. on $\Omega$ & Power (W) & Date/time & Cernox (ohms) & \multicolumn{2}{|c|}{ Cernox $(\mathrm{K})$ 4th order poly. } \\
\hline Sample ID = Cu\#1 & \multicolumn{2}{|c|}{ Beam Energy $=1.1 \mathrm{Gev}$} & \multicolumn{2}{|c|}{ Time on target $=135$ minutes } & & & & \\
\hline \multicolumn{9}{|c|}{ End of second and last irradiation at $1.1 \mathrm{GeV}$} \\
\hline & & & & & & & & \\
\hline 1.499791E-02 & $-2.216392 E-03$ & & & & $3 / 17 / 9915: 34: 33$ & & & \\
\hline$-1.499979 E-02$ & $2.211412 E-03$ & & & & $3 / 17 / 9915: 34: 36$ & & & \\
\hline$-1.499980 E-02$ & $2.211429 E-03$ & & & & $3 / 17 / 9915: 34: 39$ & & & \\
\hline $1.499794 \mathrm{E}-02$ & $-2.216391 E-03$ & & & & $3 / 17 / 9915: 34: 42$ & & & \\
\hline $1.499796 \mathrm{E}-02$ & $-2.216374 \mathrm{E}-03$ & & & & $3 / 17 / 9915: 34: 44$ & & & \\
\hline$-1.499984 \mathrm{E}-02$ & $2.211419 \mathrm{E}-03$ & & & & $3 / 17 / 9915: 34: 47$ & & & \\
\hline$-1.499982 \mathrm{E}-02$ & $2.211432 \mathrm{E}-03$ & & & & $3 / 17 / 9915: 34: 50$ & & & \\
\hline $1.499799 \mathrm{E}-02$ & $-2.216379 \mathrm{E}-03$ & & & & $3 / 17 / 9915: 34: 53$ & & & \\
\hline 1.499800E-02 & $-2.216385 \mathrm{E}-03$ & & & & $3 / 17 / 9915: 34: 56$ & & & \\
\hline$-1.499986 E-02$ & $2.211436 \mathrm{E}-03$ & & & & $3 / 17 / 9915: 34: 59$ & & & \\
\hline$-1.499987 \mathrm{E}-02$ & $2.211432 \mathrm{E}-03$ & & & & $3 / 17 / 9915: 35: 01$ & & & \\
\hline $1.499801 \mathrm{E}-02$ & $-2.216377 \mathrm{E}-03$ & & & & $3 / 17 / 9915: 35: 04$ & & & \\
\hline $1.499802 \mathrm{E}-02$ & $-2.216368 \mathrm{E}-03$ & & & & $3 / 17 / 9915: 35: 07$ & & & \\
\hline$-1.499986 \mathrm{E}-02$ & $2.211444 \mathrm{E}-03$ & & & & $3 / 17 / 9915: 35: 10$ & & & \\
\hline$-1.499989 E-02$ & $2.211456 \mathrm{E}-03$ & & & & $3 / 17 / 9915: 35: 13$ & & & \\
\hline $1.499804 \mathrm{E}-02$ & $-2.216387 \mathrm{E}-03$ & & & & $3 / 17 / 9915: 35: 16$ & & & \\
\hline $1.499806 \mathrm{E}-02$ & $-2.216379 \mathrm{E}-03$ & & & & 3/17/99 15:35:18 & & & \\
\hline$-1.499986 E-02$ & $2.211447 \mathrm{E}-03$ & & & & $3 / 17 / 9915: 35: 21$ & & & \\
\hline$-1.499985 E-02$ & $2.211446 \mathrm{E}-03$ & & & & $3 / 17 / 9915: 35: 24$ & & & \\
\hline $1.499805 E-02$ & $-2.216364 \mathrm{E}-03$ & & & & $3 / 17 / 9915: 35: 27$ & & & \\
\hline $1.499804 \mathrm{E}-02$ & $-2.216371 \mathrm{E}-03$ & & & & $3 / 17 / 99$ 15:35:30 & & & \\
\hline$-1.499987 E-02$ & $2.211448 \mathrm{E}-03$ & & & & $3 / 17 / 99$ 15:35:33 & & & \\
\hline$-1.499985 E-02$ & $2.211441 \mathrm{E}-03$ & & & & $3 / 17 / 9915: 35: 35$ & & & \\
\hline $1.499805 \mathrm{E}-02$ & $-2.216374 \mathrm{E}-03$ & & & & $3 / 17 / 9915: 35: 38$ & & & \\
\hline $1.499802 \mathrm{E}-02$ & $-2.216359 \mathrm{E}-03$ & & & & $3 / 17 / 9915: 35: 41$ & & & \\
\hline$-1 . \overline{49} 9992 \mathrm{E}-\overline{02}$ & $2.211470 \mathrm{E}-03$ & & & & $3 / 17 / 99$ 15:35:44 & & & \\
\hline$-1.499994 \mathrm{E}-02$ & $2.211471 \mathrm{E}-03$ & & & & $3 / 17 / 99 \quad 15: 35: 47$ & & & \\
\hline 1.499804E-02 & $-2.216348 \mathrm{E}-03$ & & & & $3 / 17 / 9915: 35: 50$ & & & \\
\hline 1.499802E-02 & $-2.216363 E-03$ & & & & $3 / 17 / 9915: 35: 52$ & & & \\
\hline$-1.499995 \mathrm{E}-02$ & $2.211464 \mathrm{E}-03$ & & & & $3 / 17 / 9915: 35: 55$ & & & \\
\hline$-1.499993 \mathrm{E}-02$ & $2.211463 \mathrm{E}-03$ & & & & $3 / 17 / 9915: 35: 58$ & & & \\
\hline $1.499811 \mathrm{E}-02$ & $-2.216351 E-03$ & & & & $3 / 17 / 9915: 36: 01$ & & & \\
\hline $1.499811 \mathrm{E}-02$ & $-2.216375 \mathrm{E}-03$ & & & & $3 / 17 / 9915: 36: 04$ & & & \\
\hline$-1.499994 \mathrm{E}-02$ & $2.211460 \mathrm{E}-03$ & & & & $3 / 17 / 9915: 36: 06$ & & & \\
\hline$-1.499996 \mathrm{E}-02$ & $2.211467 \mathrm{E}-03$ & \multirow{2}{*}{-} & & & $3 / 17 / 9915: 36: 09$ & & & \\
\hline $1.499812 \mathrm{E}-02$ & $-2.216370 \mathrm{E}-03$ & & & & $3 / 17 / 9915: 36: 12$ & & & \\
\hline 1.499811E-02 & $-2.216371 E-03$ & & & & $3 / 17 / 99$ 15:36:15 & & & \\
\hline$-1.500000 \mathrm{E}-02$ & $2.211479 \mathrm{E}-03$ & & & & 3/17/99 15:36:18 & & & \\
\hline$-1.500002 E-02$ & $2.211481 \mathrm{E}-03$ & & & & $3 / 17 / 99$ 15:36:21 & & & \\
\hline $1.499808 \mathrm{E}-02$ & $-2.216348 E-03$ & & & & $3 / 17 / 9915: 36: 23$ & & & \\
\hline $1.499896 \mathrm{E}-02$ & $2.213911 \mathrm{E}-03$ & 0.1476042 & 4.021889E-07 & $3.320661 E-05$ & $3 / 17 / 99$ 15:36:28 & 4977.053861 & 4.726435783 & \\
\hline & & & & & & & & \\
\hline & & & & & & & & \\
\hline
\end{tabular}


Copper $1 . x / s$

\begin{tabular}{|c|c|c|c|c|c|c|c|c|}
\hline Current $(A)$ & Voltage (V) & Res.(ohms) & Std. Dev. on $\Omega$ & Power (W) & Date/time & Cernox (ohms) & \multicolumn{2}{|c|}{ Cernox (K) 4th order poly. } \\
\hline Sample ID= Cu\#1 & \multicolumn{3}{|c|}{ Beam Energy $=0 \quad$ Time on target $=0$} & & & & & \\
\hline \multicolumn{9}{|c|}{ Post irradiation resistance measurements at ambient temperature in the U-line } \\
\hline & & & & & & & & \\
\hline $5.000000 \mathrm{E}-05$ & $-8.970000 \mathrm{E}-04$ & & & & 3/19/99 13:44:00 & & & \\
\hline$-5.000000 E-05$ & $8.970000 \mathrm{E}-04$ & & & & $3 / 19 / 9913: 44: 00$ & & & \\
\hline$-5.000000 \mathrm{E}-05$ & $8.970000 \mathrm{E}-04$ & & & & $3 / 19 / 9913: 44: 00$ & & & \\
\hline $5.000000 \mathrm{E}-05$ & $-8.970000 \mathrm{E}-04$ & & & & 3/19/99 13:44:00 & & & \\
\hline $5.000000 E-05$ & $-8.970000 \mathrm{E}-04$ & & & & $3 / 19 / 99 \quad 13: 44: 00$ & & & \\
\hline$-5.000000 \mathrm{E}-05$ & $8.970000 \mathrm{E}-04$ & & & & 3/19/99 13:45:00 & & & \\
\hline$-5.000000 E-05$ & $8.970000 \mathrm{E}-04$ & & & & $3 / 19 / 9913: 45: 00$ & & & \\
\hline $5.000000 \mathrm{E}-05$ & $-8.970000 \mathrm{E}-04$ & & & & 3/19/99 13:45:00 & & & \\
\hline $5.000000 \mathrm{E}-05$ & $-8.970000 \mathrm{E}-04$ & & & & $3 / 19 / 9913: 45: 00$ & & & \\
\hline-5.000000505 & $8.970000 E-04$ & & & & $3 / 19 / 9913: 45: 00$ & & & \\
\hline$-5.000000 E-05$ & $8.970000 \mathrm{E}-04$ & & & & $3 / 19 / 9913: 45: 00$ & & & \\
\hline $5.000000 \mathrm{E}-05$ & $-8.970000 E-04$ & & & & 3/19/99 13:45:00 & & & \\
\hline $5.000000 \mathrm{E}-05$ & $-8.970000 E-04$ & & & & 3/19/99 13:45:00 & & & \\
\hline$-5.000000 \mathrm{E}-05$ & $8.970000 \mathrm{E}-04$ & & & & $3 / 19 / 99$ 13:45:00 & & & \\
\hline$-5.000000 E-05$ & 8.970000 E-04 & & & & $3 / 19 / 9913: 45: 00$ & & & \\
\hline $5.000000 \mathrm{E}-05$ & $-8.970000 \mathrm{E}-04$ & & & & $3 / 19 / 99 \quad 13: 45: 00$ & & & \\
\hline $5.000000 \mathrm{E}-05$ & $-8.970000 \mathrm{E}-04$ & & & & $3 / 19 / 99$ 13:45:00 & & & \\
\hline-5.000000 E-05 & $8.970000 \mathrm{E}-04$ & & & & 3/19/99 13:45:00 & & & \\
\hline$-5.000000 \mathrm{E}-05$ & 8.970000 E-04 & & & & 3/19/99 13:45:00 & & & \\
\hline $5.000000 \mathrm{E}-05$ & $-8.970000 \mathrm{E}-04$ & & & & $3 / 19 / 9913: 45: 00$ & & & \\
\hline $5.000000 \mathrm{E}-05$ & $-8.970000 E-04$ & & & & $3 / 19 / 9913: 45: 00$ & & & \\
\hline$-5.000000 \mathrm{E}-05$ & $8.970000 \mathrm{E}-04$ & & & & $3 / 19 / 99$ 13:45:00 & & & \\
\hline$-5.000000 \mathrm{E}-05$ & 8.970000 E-04 & & & & $3 / 19 / 99$ 13:45:00 & & & \\
\hline $5.000000 \mathrm{E}-05$ & $-8.970000 E-04$ & & & & $3 / 19 / 99$ 13:45:00 & & & \\
\hline $5.000000 \mathrm{E}-05$ & $-8.970000 \mathrm{E}-04$ & & & & $3 / 19 / 9913: 45: 00$ & & & \\
\hline$-5.000000 \mathrm{E}-05$ & $8.970000 \mathrm{E}-04$ & & & & $3 / 19 / 9913: 45: 00$ & & & \\
\hline$-5.000000 \mathrm{E}-05$ & $8.970000 \mathrm{E}-04$ & & & & 3/19/99 13:45:00 & & & \\
\hline $5.000000 \mathrm{E}-05$ & $-8.970000 E-04$ & & & & $3 / 19 / 9913: 46: 00$ & & & \\
\hline $5.000000 E-05$ & $-8.970000 E-04$ & & & & $3 / 19 / 9913: 46: 00$ & & & \\
\hline$-5.000000 \mathrm{E}-05$ & $8.970000 E-04$ & & & & $3 / 19 / 9913: 46: 00$ & & & \\
\hline$-5.000000 \mathrm{E}-05$ & $8.970000 E-04$ & & & & $3 / 19 / 9913: 46: 00$ & & & \\
\hline $5.000000 \mathrm{E}-05$ & $-8.970000 \mathrm{E}-04$ & & & & $3 / 19 / 9913: 46: 00$ & & & \\
\hline $5.000000 \mathrm{E}-05$ & -8.970000 E-04 & & & & $3 / 19 / 9913: 46: 00$ & & & \\
\hline$-5.000000 \mathrm{E}-05$ & $8.970000 E-04$ & & & & $3 / 19 / 99$ 13:46:00 & & & \\
\hline$-5.000000 \mathrm{E}-05$ & $8.970000 E-04$ & & & & 3/19/99 13:46:00 & & & \\
\hline $5.000000 \mathrm{E}-05$ & $-8.970000 \mathrm{E}-04$ & & & & $3 / 19 / 99$ 13:46:00 & & & \\
\hline $5.000000 E-05$ & -8.970000 E-04 & & & & $3 / 19 / 9913: 46: 00$ & & & \\
\hline$-5.000000 \mathrm{E}-05$ & 8.970000 E-04 & & & & $3 / 19 / 9913: 46: 00$ & & & \\
\hline$-5.000000 \mathrm{E}-05$ & $8.970000 \mathrm{E}-04$ & & & & $3 / 19 / 9913: 46: 00$ & & & \\
\hline $5.000000 \mathrm{E}-05$ & $-8.970000 \mathrm{E}-04$ & & & & $3 / 19 / 9913: 46: 00$ & & & \\
\hline $5.000000 \mathrm{E}-05$ & $8.972050 \mathrm{E}-04$ & 17.9428937 & $6.000680 \mathrm{E}-04$ & $4.490000 \mathrm{E}-08$ & $3 / 19 / 9913: 46: 00$ & 60.6461358 & 290.1081583 & \\
\hline & & & & & & & & \\
\hline
\end{tabular}


Copper1.x/s

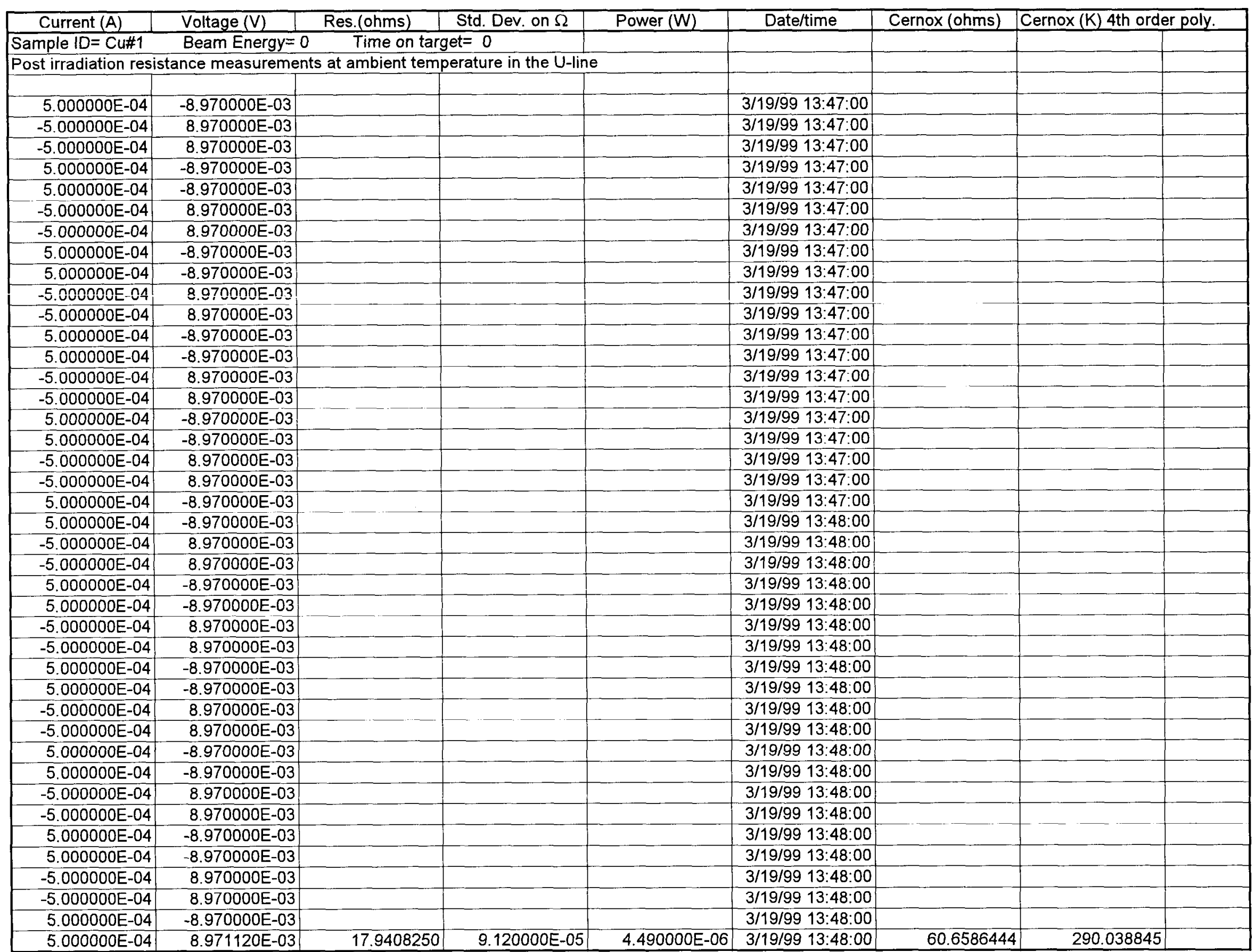


COPPER1.XIs

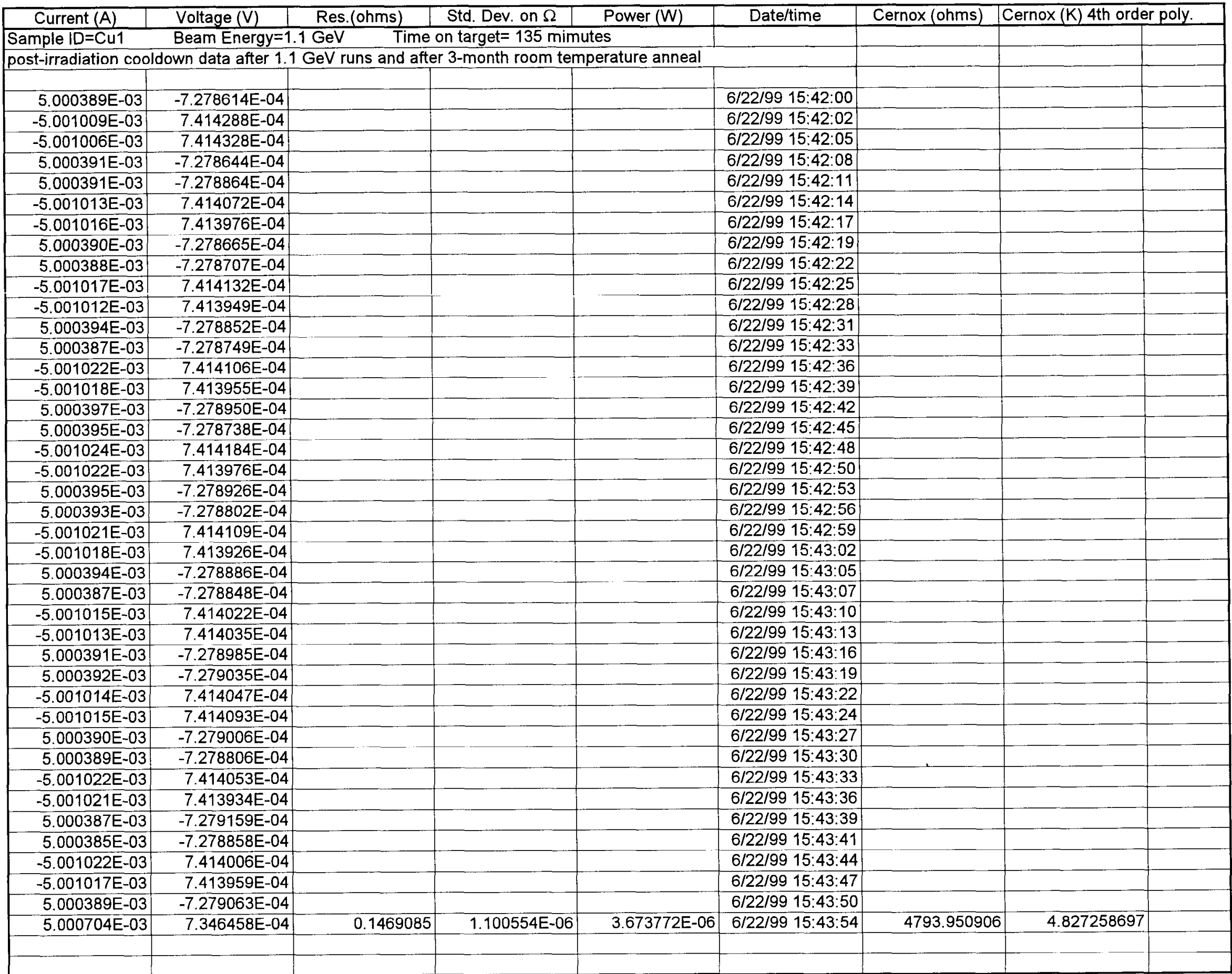


COPPER 1.xls

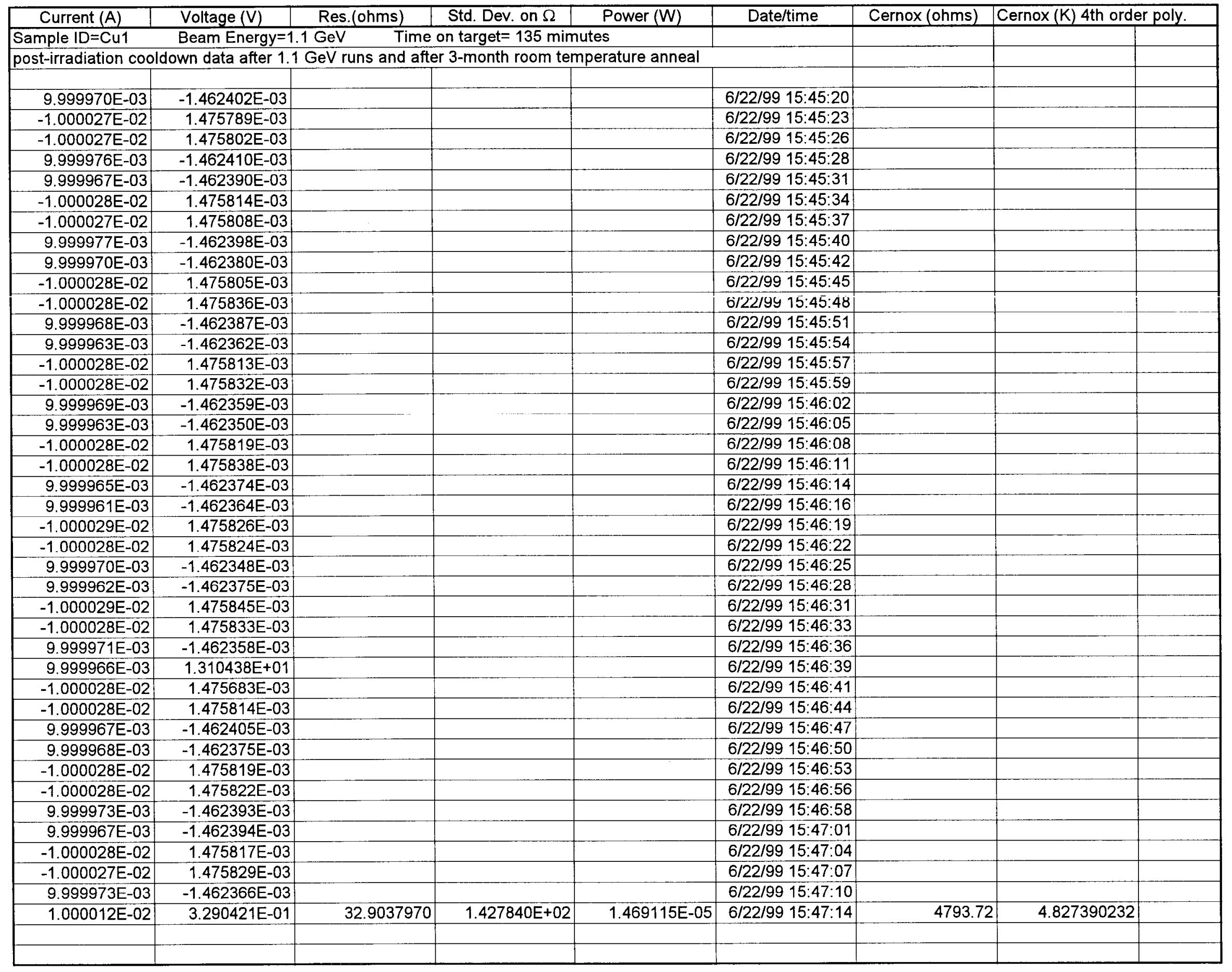


COPPER1.xls

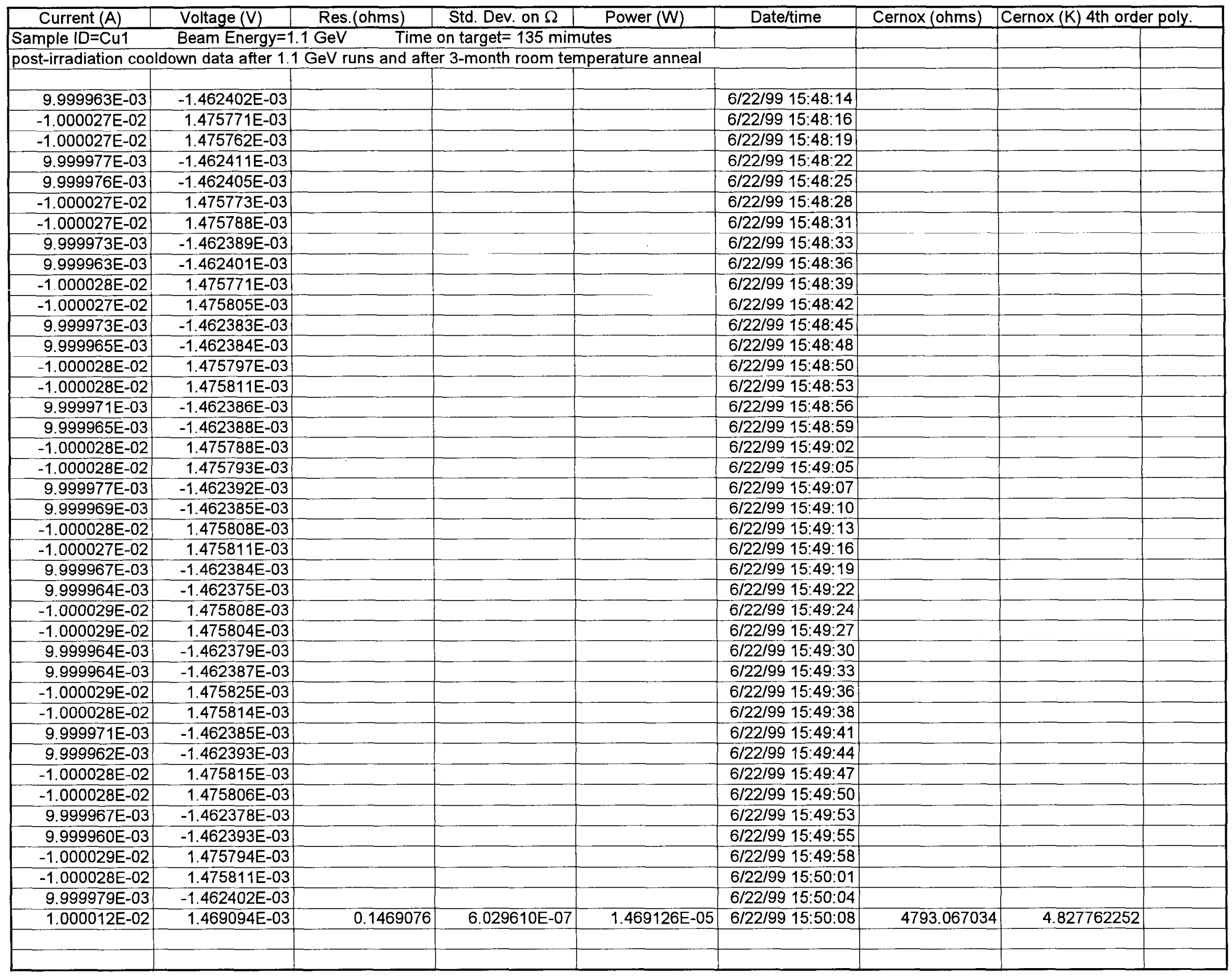


COPPER1.xls

\begin{tabular}{|c|c|c|c|c|c|c|c|c|}
\hline Current $(A)$ & Voltage (V) & Res.(ohms) & Std. Dev. on $\Omega$ & Power (W) & Date/time & Cernox (ohms) & \multicolumn{2}{|c|}{ Cernox (K) 4th order poly. } \\
\hline Sample ID=Cu1 & \multicolumn{4}{|c|}{ Time on target $=135$ mimutes } & & & & \\
\hline \multicolumn{9}{|c|}{ post-irradiation cooldown data after $1.1 \mathrm{GeV}$ runs and after 3 -month room temperature anneal } \\
\hline & & & & & & & & \\
\hline $1.500124 \mathrm{E}-02$ & $-2.197149 \mathrm{E}-03$ & & & & $6 / 22 / 9915: 50: 58$ & & & \\
\hline$-1.500674 \mathrm{E}-02$ & $2.211221 \mathrm{E}-03$ & & & & $6 / 22 / 9915: 51: 01$ & & & \\
\hline$-1.500676 \mathrm{E}-02$ & $2.211201 E-03$ & & & & $6 / 22 / 9915: 51: 04$ & & & \\
\hline $1.500137 \mathrm{E}-02$ & $-2.197173 \mathrm{E}-03$ & & & & $6 / 22 / 99$ 15:51:06 & & & \\
\hline $1.500141 \mathrm{E}-02$ & $-2.197171 \mathrm{E}-03$ & & & & $6 / 22 / 99$ 15:51:09 & & & \\
\hline$-1.500663 \mathrm{E}-02$ & $2.211188 \mathrm{E}-03$ & & & & $6 / 22 / 99 \quad 15: 51: 12$ & & & \\
\hline$-1.500659 \mathrm{E}-02$ & $2.211204 E-03$ & & & & $6 / 22 / 99$ 15:51:15 & & & \\
\hline $1.500138 \mathrm{E}-02$ & $-2.197143 E-03$ & & & & $6 / 22 / 99$ 15:51:18 & & & \\
\hline $1.500139 \mathrm{E}-02$ & $-2.197161 \mathrm{E}-03$ & & & & $6 / 22 / 99$ 15:51:21 & & & \\
\hline$-1.500664 \mathrm{E}-02$ & $2.211224 \mathrm{E}-03$ & & & & $6 / 22 / 99$ 15:51:23 & & & \\
\hline$-1.500669 \mathrm{E}-02$ & $2.211233 \mathrm{E}-03$ & & & & $6 / 22 / 99$ 15:51:26 & & & \\
\hline $1.500141 E-02$ & $-2.197147 \mathrm{E}-03$ & & & & $6 / 22 / 99$ 15:51:29 & & & \\
\hline $1.500147 \mathrm{E}-02$ & $-2.197156 \mathrm{E}-03$ & & & & $6 / 22 / 99$ 15:51:32 & & & \\
\hline$-1.500660 \mathrm{E}-02$ & $2.211218 \mathrm{E}-03$ & & & & $6 / 22 / 99$ 15:51:35 & & & \\
\hline$-1.500665 \mathrm{E}-02$ & $2.211239 \mathrm{E}-03$ & & & & $6 / 22 / 9915: 51: 38$ & & & \\
\hline $1.500142 E-02$ & $-2.197124 E-03$ & & & & $6 / 22 / 9915: 51: 40$ & & & \\
\hline $1.500145 \mathrm{E}-02$ & $-2.197160 \mathrm{E}-03$ & & & & $6 / 22 / 9915: 51: 43$ & & & \\
\hline$-1.500660 \mathrm{E}-02$ & $2.211223 E-03$ & & & & $6 / 22 / 9915: 51: 46$ & & & \\
\hline$-1.500657 \mathrm{E}-02$ & $2.211225 E-03$ & & & & $6 / 22 / 9915: 51: 49$ & & & \\
\hline $1.500139 \mathrm{E}-02$ & $-2.197138 \mathrm{E}-03$ & & & & $6 / 22 / 99$ 15:51:52 & & & \\
\hline 1.500139E-02 & $-2.197166 \mathrm{E}-03$ & & & & $6 / 22 / 9915: 51: 54$ & & & \\
\hline$-1.500664 \mathrm{E}-02$ & $2.211246 \mathrm{E}-03$ & & & & $6 / 22 / 9915: 51: 57$ & & & \\
\hline$-1.500663 \mathrm{E}-02$ & $2.211211 \mathrm{E}-03$ & & & & $6 / 22 / 99$ 15:52:00 & & & \\
\hline $1.500143 \mathrm{E}-02$ & $-2.197141 E-03$ & & & & $6 / 22 / 9915: 52: 03$ & & & \\
\hline $1.500142 E-02$ & $-2.197133 \mathrm{E}-03$ & & & & $6 / 22 / 9915: 52: 06$ & & & \\
\hline$-1.500663 \mathrm{E}-02$ & $2.211227 \mathrm{E}-03$ & & & & $6 / 22 / 9915: 52: 09$ & & & \\
\hline$-1.500666 E-02$ & $2.211235 \mathrm{E}-03$ & & & & $6 / 22 / 9915: 52: 11$ & & & \\
\hline $1.500136 \mathrm{E}-02$ & $-2.197118 \mathrm{E}-03$ & & & & $6 / 22 / 99$ 15:52:14 & & & \\
\hline $1.500145 \mathrm{E}-02$ & $-2.197117 \mathrm{E}-03$ & & & & $6 / 22 / 99$ 15:52:17 & & & \\
\hline$-1.500664 \mathrm{E}-02$ & $2.211241 \mathrm{E}-03$ & & & & $6 / 22 / 99 \quad 15: 52: 20$ & & & \\
\hline$-1.500661 \mathrm{E}-02$ & $2.211247 \mathrm{E}-03$ & & & & $6 / 22 / 99$ 15:52:23 & & & \\
\hline $1.500141 \mathrm{E}-02$ & $-2.197134 \mathrm{E}-03$ & & & & $6 / 22 / 99$ 15:52:26 & & & \\
\hline $1.500145 \mathrm{E}-02$ & $-2.197135 \mathrm{E}-03$ & & & & $6 / 22 / 9915: 52: 28$ & & & \\
\hline$-1.500655 \mathrm{E}-02$ & $2.211185 E-03$ & & & & $6 / 22 / 9915: 52: 31$ & & & \\
\hline$-1.500654 \mathrm{E}-02$ & $2.211207 \mathrm{E}-03$ & & & & $6 / 22 / 99$ 15:52:34 & & & \\
\hline $1.500141 \mathrm{E}-02$ & $-2.197142 \mathrm{E}-03$ & & & & $6 / 22 / 99$ 15:52:37 & & & \\
\hline $1.500140 \mathrm{E}-02$ & $-2.197166 \mathrm{E}-03$ & & & & $6 / 22 / 99$ 15:52:40 & & & \\
\hline$-1.500659 \mathrm{E}-02$ & $2.211197 \mathrm{E}-03$ & & & & $6 / 22 / 9915: 52: 43$ & & & \\
\hline$-1.500660 \mathrm{E}-02$ & $2.211245 E-03$ & & & & $6 / 22 / 9915: 52: 45$ & & & \\
\hline $1.500145 \mathrm{E}-02$ & $-2.197149 \mathrm{E}-03$ & & & & $6 / 22 / 99$ 15:52:48 & & & \\
\hline $1.500402 \mathrm{E}-02$ & $2.204184 \mathrm{E}-03$ & 0.1469062 & $5.964840 \mathrm{E}-07$ & $3.307182 \mathrm{E}-05$ & $6 / 22 / 9915: 52: 52$ & 4792.657526 & 4.827995612 & \\
\hline
\end{tabular}


APPENDIX 3

EXCEL IDATA FILE OF RESISTANCE MEASUREMENTS OF COPPER SAMPLE \#2 


\begin{tabular}{|c|c|c|c|c|c|c|c|c|}
\hline Current (A) & Voltage (V) & Res.(ohms) & Std. Dev. Of $\Omega$ & Power (W) & Date/time & Cernox (ohms) & Cernox (K) 4th orde & er poly. \\
\hline Sample ID= Cu\#2 & Beam Energy $=$ & Time on tar & rget $=0$ & & & & & \\
\hline Pre-irradiation resis & stivity data at room & temperature prior to & to testing at $1.94 \mathrm{Ge}$ & & & & & \\
\hline & & & & & & & & \\
\hline $5.000259 \mathrm{E}-05$ & $-8.657660 \mathrm{E}-04$ & & & & $3 / 16 / 99$ 4:37:18 & & & \\
\hline$-5.000510 \mathrm{E}-05$ & $8.659028 E-04$ & & & & $3 / 16 / 994: 37: 21$ & & & \\
\hline$-5.000513 E-05$ & $8.659079 \mathrm{E}-04$ & & & & $3 / 16 / 994: 37: 23$ & & & \\
\hline $5.000263 \mathrm{E}-05$ & $-8.657481 E-04$ & & & & $3 / 16 / 994: 37: 26$ & & & \\
\hline $5.000261 \mathrm{E}-05$ & $-8.657446 \mathrm{E}-04$ & & & & $3 / 16 / 994: 37: 29$ & & & \\
\hline$-5.000514 \mathrm{E}-05$ & $8.659243 E-04$ & & & & $3 / 16 / 994: 37: 32$ & & & \\
\hline$-5.000515 \mathrm{E}-05$ & $8.659352 E-04$ & & & & $3 / 16 / 994: 37: 35$ & & & \\
\hline $5.000263 \mathrm{E}-05$ & $-8.657223 \mathrm{E}-04$ & & & & $3 / 16 / 994: 37: 38$ & & & \\
\hline $5.000263 \mathrm{E}-05$ & $-8.657177 \mathrm{E}-04$ & & & & $3 / 16 / 994: 37: 40$ & & & \\
\hline$-5.000514 E-05$ & $8.659471 E-04$ & & & & $3 / 16 / 994: 37: 43$ & & & \\
\hline$-5.000512 E-05$ & $8.659476 \mathrm{E}-04$ & & & & $3 / 16 / 994: 37: 46$ & & & \\
\hline $5.000264 \mathrm{E}-05$ & $-8.657050 \mathrm{E}-04$ & & & & $3 / 16 / 994: 37: 49$ & & & \\
\hline $5.000267 \mathrm{E}-05$ & $-8.657146 \mathrm{E}-04$ & & & & $3 / 16 / 994: 37: 52$ & & & \\
\hline$-5.000515 E-05$ & $8.659608 \mathrm{E}-04$ & & & & $3 / 16 / 994: 37: 55$ & & & \\
\hline$-5.000518 \mathrm{E}-05$ & $8.659583 \mathrm{E}-04$ & & & & $3 / 16 / 994: 37: 57$ & & & \\
\hline $5.000264 \mathrm{E}-05$ & $-8.656872 \mathrm{E}-04$ & & & & $3 / 16 / 994: 38: 00$ & & & \\
\hline $5.000263 \mathrm{E}-05$ & $-8.656959 E-04$ & & & & 3/16/99 4:38:03 & & & \\
\hline$-5.000516 \mathrm{E}-05$ & $8.659776 \mathrm{E}-04$ & & & & $3 / 16 / 994: 38: 06$ & & & \\
\hline$-5.000521 E-05$ & $8.659770 \mathrm{E}-04$ & & & & $3 / 16 / 994: 38: 09$ & & & \\
\hline 5.000259E-05 & $-8.656680 \mathrm{E}-04$ & & & & $3 / 16 / 994: 38: 12$ & & & \\
\hline $5.000262 \mathrm{E}-05$ & $-8.656831 E-04$ & & & & $3 / 16 / 994: 38: 14$ & & & \\
\hline$-5.000521 \mathrm{E}-05$ & $8.659771 \mathrm{E}-04$ & & & & $3 / 16 / 994: 38: 17$ & & & \\
\hline$-5.000519 E-05$ & $8.659776 \mathrm{E}-04$ & & & & $3 / 16 / 994: 38: 20$ & & & \\
\hline $5.000263 \mathrm{E}-05$ & $-8.656800 \mathrm{E}-04$ & & & & $3 / 16 / 994: 38: 23$ & & & \\
\hline $5.000260 \mathrm{E}-05$ & $-8.656743 \mathrm{E}-04$ & & & & 3/16/99 4:38:26 & & & \\
\hline$-5.000520 \mathrm{E}-05$ & $8.659876 \mathrm{E}-04$ & & & & $3 / 16 / 99$ 4:38:28 & & & \\
\hline$-5.000518 \mathrm{E}-05$ & 8.659964E-04 & & & & $3 / 16 / 994: 38: 31$ & & & \\
\hline $5.000261 E-05$ & $-8.656846 \mathrm{E}-04$ & & & & $3 / 16 / 994: 38: 34$ & & & \\
\hline $5.000263 \mathrm{E}-05$ & $-8.656633 \mathrm{E}-04$ & & & & $3 / 16 / 994: 38: 37$ & & & \\
\hline$-5.000516 \mathrm{E}-05$ & $8.659989 \mathrm{E}-04$ & & & & $3 / 16 / 994: 38: 40$ & & & \\
\hline$-5.000512 E-05$ & $8.659965 E-04$ & & & & $3 / 16 / 994: 38: 43$ & & & \\
\hline $5.000264 \mathrm{E}-05$ & $-8.656540 \mathrm{E}-04$ & & & & $3 / 16 / 994: 38: 45$ & & & \\
\hline $5.000269 \mathrm{E}-05$ & $-8.656528 \mathrm{E}-04$ & & & & $3 / 16 / 994: 38: 48$ & & & \\
\hline$-5.000513 E-05$ & 8.659988E-04 & & & & $3 / 16 / 994: 38: 51$ & & & \\
\hline$-5.000512 E-05$ & $8.660099 E-04$ & & & & $3 / 16 / 994: 38: 54$ & & & \\
\hline $5.000271 \mathrm{E}-05$ & $-8.656538 \mathrm{E}-04$ & & & & $3 / 16 / 994: 38: 57$ & & & \\
\hline $5.000271 \mathrm{E}-05$ & $-8.656548 \mathrm{E}-04$ & & & & $3 / 16 / 99$ 4:39:00 & & & \\
\hline$-5.000520 \mathrm{E}-05$ & $8.660288 E-04$ & & & & $3 / 16 / 99$ 4:39:02 & & & \\
\hline$-5.000517 \mathrm{E}-05$ & $8.660226 \mathrm{E}-04$ & & & & 3/16/99 4:39:05 & & & \\
\hline $5.000274 \mathrm{E}-05$ & $-8.656396 \mathrm{E}-04$ & & & & 3/16/99 4:39:08 & & & \\
\hline $5.000390 \mathrm{E}-05$ & $8.658311 \mathrm{E}-04$ & 17.3152706 & $1.041166 \mathrm{E}-04$ & 4.329498E-08 & $3 / 16 / 994: 39: 12$ & 60.50701688 & 290.8810806 & \\
\hline & & & & & & & & \\
\hline
\end{tabular}


Copper2.xls

\begin{tabular}{|c|c|c|c|c|c|c|c|c|}
\hline Current (A) & Voltage (V) & Res.ohms) & Std. Dev. Of $\Omega$ & Power (W) & Date/time & Cernox (ohms) & \multicolumn{2}{|c|}{ Cernox $(K)$ 4th order poly. } \\
\hline Sample ID= Cu\#2 & Beam Energy= & Time on tar & rget $=0$ & & & & & \\
\hline \multicolumn{9}{|c|}{ Pre-irradiation resistivity data at room temperature prior to testing at $1.94 \mathrm{GeV}$} \\
\hline & & & & & & & & \\
\hline $5.000234 E-04$ & $-8.657561 \mathrm{E}-03$ & & & & $3 / 16 / 994: 40: 15$ & & & \\
\hline$-5.000655 E-04$ & 8.657989E-03 & & & & $3 / 16 / 994: 40: 18$ & & & \\
\hline$-5.000649 \mathrm{E}-04$ & $8.658011 \mathrm{E}-03$ & & & & $3 / 16 / 994: 40: 21$ & & & \\
\hline 5.000233E-04 & $-8.657548 E-03$ & & & & $3 / 16 / 994: 40: 24$ & & & \\
\hline $5.000231 E-04$ & $-8.657568 \mathrm{E}-03$ & \multirow[t]{2}{*}{ 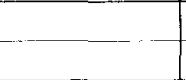 } & & & $3 / 16 / 994: 40: 26$ & & & \\
\hline$-5.000649 \mathrm{E}-04$ & $8.658045 \mathrm{E}-03$ & & & & $3 / 16 / 994: 40: 29$ & & & \\
\hline$-5.000646 \mathrm{E}-04$ & $8.658015 \mathrm{E}-03$ & & & & $3 / 16 / 994: 40: 32$ & & & \\
\hline $5.000239 E-04$ & $-8.657554 \mathrm{E}-03$ & & & & $3 / 16 / 994: 40: 35$ & & & \\
\hline $5.000239 \mathrm{E}-04$ & $-8.657574 \mathrm{E}-03$ & & & & $3 / 16 / 994: 40: 38$ & & & \\
\hline$-5.000648 E-04$ & $8.658040 \mathrm{E}-03$ & & & & $3 / 16 / 994: 40: 41$ & & & \\
\hline$-5.000652 E-04$ & $8.658041 \mathrm{E}-03$ & & & & $3 / \overline{16 / 99} 4: \overline{40: 43}$ & & & \\
\hline $5.000241 E-04$ & $-8.657552 \mathrm{E}-03$ & & & & $3 / 16 / 994: 40: 46$ & & & \\
\hline $5.000244 E-04$ & $-8.657565 E-03$ & & & & $3 / 16 / 994: 40: 49$ & & & \\
\hline$-5.000655 E-04$ & $8.658084 \mathrm{E}-03$ & & & & $3 / 16 / 994: 40: 52$ & & & \\
\hline$-5.000654 \mathrm{E}-04$ & $8.658066 \mathrm{E}-03$ & & & & $3 / 16 / 994: 40: 55$ & & & \\
\hline $5.000241 \mathrm{E}-04$ & $-8.657569 \mathrm{E}-03$ & & & & $3 / 16 / 994: 40: 58$ & & & \\
\hline $5.000242 E-04$ & $-8.657547 \mathrm{E}-03$ & & & & $3 / 16 / 994: 41: 00$ & & & \\
\hline$-5.000658 E-04$ & $8.658082 E-03$ & & & & $3 / 16 / 994: 41: 03$ & & & \\
\hline$-5.000652 \mathrm{E}-04$ & $8.658084 E-03$ & & & & $3 / 16 / 994: 41: 06$ & & & \\
\hline $5.000232 \mathrm{E}-04$ & $-8.657531 E-03$ & & & & $3 / 16 / 994: 41: 09$ & \multirow[t]{2}{*}{ 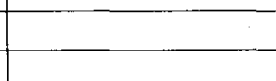 } & & \\
\hline $5.000231 \mathrm{E}-04$ & $-8.657522 E-03$ & & & & $3 / 1 \overline{6} / 994: 4 \overline{1}: 12$ & & & \\
\hline$-5.000653 \mathrm{E}-04$ & $8.658074 \mathrm{E}-03$ & & & & $3 / 16 / 994: 41: 15$ & & & \\
\hline$-5.000648 E-04$ & $8.658102 \mathrm{E}-03$ & & & & $3 / 16 / 994: 41: 17$ & & & \\
\hline $5.000237 \mathrm{E}-04$ & $-8.657554 E-03$ & & & & $3 / 16 / 994: 41: 20$ & & & \\
\hline $5.000238 \mathrm{E}-04$ & $-8.657530 \mathrm{E}-03$ & & & & $3 / 16 / 994: 41: 23$ & & & \\
\hline$-5.000652 E-04$ & $8.658100 \mathrm{E}-03$ & & & & $3 / 16 / 994: 4 \overline{1}: 26$ & & & \\
\hline$-5.000651 \mathrm{E}-04$ & $8.658126 \mathrm{E}-03$ & & & & $3 / 16 / 994: 41: 29$ & & & \\
\hline $5.000233 E-04$ & $-8.657490 \mathrm{E}-03$ & & & & $3 / 16 / 994: 41: 32$ & & & \\
\hline $5.000232 E-04$ & $-8.657522 E-03$ & & & & $3 / 16 / 994: 41: 34$ & \multirow{3}{*}{$\begin{array}{ll}- & -\end{array}$} & & \\
\hline$-5.000652 \mathrm{E}-04$ & $8.658099 E-03$ & & & & $3 / 16 / 994: 41: 37$ & & \multirow{2}{*}{-} & \\
\hline$-5.000649 \mathrm{E}-04$ & $8.658130 \mathrm{E}-03$ & & & & $3 / 16 / 994: 41: 40$ & & & \\
\hline $5.000234 \mathrm{E}-04$ & $-8.657542 E-03$ & & & & $3 / 16 / 994: 41: 43$ & & & \\
\hline $5.000239 E-04$ & $-8.657544 \mathrm{E}-03$ & & & & $3 / 16 / 994: 41: 46$ & & & \\
\hline$-5.000652 \mathrm{E}-04$ & $8.658106 \mathrm{E}-03$ & & & & $3 / 16 / 994: 41: 48$ & & & \\
\hline$-5.000655 E-04$ & $8.658119 \mathrm{E}-03$ & & & & $3 / 16 / 994: 41: 51$ & & & \\
\hline $5.000237 \mathrm{E}-04$ & $-8.657525 E-03$ & & & & $3 / 16 / 994: 41: 54$ & & & \\
\hline $5.000241 E-04$ & $-8.657544 \mathrm{E}-03$ & & & & $3 / 16 / 994: 41: 57$ & & & \\
\hline$-5.000657 \mathrm{E}-04$ & $8.658108 \mathrm{E}-03$ & & & & $3 / 16 / 994: 42: 00$ & & & \\
\hline$-5.000656 \mathrm{E}-04$ & $8.658111 \mathrm{E}-03$ & & & & 3/16/99 4:42:03 & & & \\
\hline $5.000238 E-04$ & $-8.657520 \mathrm{E}-03$ & & & & $3 / 16 / 994: 42: 05$ & & & \\
\hline $5.000444 \mathrm{E}-04$ & $8.657810 E-03$ & 17.3140807 & $3.008804 \mathrm{E}-05$ & 4.329294E-06 & $3 / 16 / 994: 42: 10$ & 60.53214081 & 290.7412201 & \\
\hline & & & & & & & & \\
\hline
\end{tabular}

Page 3

Copper2.xis 
Copper2.xls

\begin{tabular}{|c|c|c|c|c|c|c|c|c|}
\hline Current (A) & Voltage (V) & Res.(ohms) & Std. Dev. Of $\Omega$ & Power (W) & Date/time & Cernox (ohms) & Cernox $(\mathrm{K})$ 4th orde & er poly. \\
\hline Sample ID = Cu\#2 & Beam Energy= & $=0 \quad$ Time on tar & rget $=0$ & & & & & \\
\hline Pre-irradiation resi & stivity data at liquid & helium temperature & prior to testing at 1 & $1.94 \mathrm{GeV}$ & & & & \\
\hline & & & & & & & & \\
\hline $5.000195 \mathrm{E}-03$ & $-6.988397 \mathrm{E}-04$ & & & & 3/16/99 6:18:21 & & & \\
\hline$-5.000706 \mathrm{E}-03$ & $6.836869 \mathrm{E}-04$ & & & & $3 / 16 / 996: 18: 23$ & & & \\
\hline$-5.000706 \mathrm{E}-03$ & $6.836767 \mathrm{E}-04$ & & & & $3 / 16 / 996: 18: 26$ & & & \\
\hline 5.000203E-03 & $-6.988511 E-04$ & & & & $3 / 16 / 996: 18: 29$ & & & \\
\hline 5.000198E-03 & $-6.988559 \mathrm{E}-04$ & & & & $3 / 16 / 996: 18: 32$ & & & \\
\hline$-5.000707 E-03$ & $6.836998 \mathrm{E}-04$ & & & & $3 / 16 / 996: 18: 35$ & & & \\
\hline$-5.000710 \mathrm{E}-03$ & $6.836975 \mathrm{E}-04$ & & & & 3/16/99 6:18:38 & & & \\
\hline $5.000202 E-03$ & $-6.988376 \mathrm{E}-04$ & & & & $3 / 16 / 996: 18: 40$ & & & \\
\hline $5.000204 E-03$ & $-6.988168 \mathrm{E}-04$ & & & & $3 / 16 / 996: 18: 43$ & & & \\
\hline$-5.000707 E-03$ & $6.837132 E-04$ & & & & $3 / 16 / 996: 18: 46$ & & & \\
\hline$-5.000707 \mathrm{E}-03$ & $6.837052 \mathrm{E}-04$ & & & & $3 / 16 / 996: 18: 49$ & & & \\
\hline $5.000201 \mathrm{E}-03$ & $-6.988175 E-04$ & & & & $3 / 16 / 996: 18: 52$ & & & \\
\hline 5.000205E-03 & $-6.988097 \mathrm{E}-04$ & & & & $3 / 16 / 996: 18: 54$ & & & \\
\hline$-5.000709 \mathrm{E}-03$ & $6.837025 E-04$ & & & & $3 / 16 / 996: 18: 57$ & & & \\
\hline$-5.000707 \mathrm{E}-03$ & $6.837269 \mathrm{E}-04$ & & & & $3 / 16 / 996: 19: 00$ & & & \\
\hline $5000202 \mathrm{~F}-03$ & $-6.988070 \mathrm{E}-04$ & & & & $3 / 16 / 996: 19: 03$ & & & \\
\hline $5.000200 \mathrm{E}-03$ & $-6.988265 \mathrm{E}-04$ & & & & $3 / 16 / 996: 19: 06$ & & & \\
\hline$-5.000706 \mathrm{E}-03$ & $6.837251 \mathrm{E}-04$ & & & & 3/16/99 6:19:09 & & & \\
\hline$-5.000707 \mathrm{E}-03$ & $6.837245 E-04$ & & & & 3/16/99 6:19:11 & & & \\
\hline $5.000202 \mathrm{E}-03$ & $-6.988087 E-04$ & & & & $3 / 16 / 996: 19: 14$ & & & \\
\hline $5.000200 \mathrm{E}-03$ & $-6.987994 \mathrm{E}-04$ & & & & 3/16/99 6:19:17 & & & \\
\hline$-5.000709 E-03$ & $6.837092 E-04$ & & & & $3 / 16 / 996: 19: 20$ & & & \\
\hline$-5.000706 \mathrm{E}-03$ & $6.837150 \mathrm{E}-04$ & & & & $3 / 16 / 996: 19: 23$ & & & \\
\hline $5.000202 E-03$ & $-6.988158 E-04$ & & & & $3 / 16 / 996: 19: 26$ & & & \\
\hline $5.000202 E-03$ & $-6.988200 \mathrm{E}-04$ & & & & $3 / 16 / 996: 19: 28$ & & & \\
\hline$-5.000709 \mathrm{E}-03$ & $6.837150 \mathrm{E}-04$ & & & & $3 / 16 / 996: 19: 31$ & & & \\
\hline$-5.000705 \mathrm{E}-03$ & $6.837181 \mathrm{E}-04$ & & & & $3 / 16 / 996: 19: 34$ & & & \\
\hline $5.000205 \mathrm{E}-03$ & $-6.988172 \mathrm{E}-04$ & & & & $3 / 16 / 996: 19: 37$ & & & \\
\hline 5.000203E-03 & $-6.988309 \mathrm{E}-04$ & & & & $3 / 16 / 996: 19: 40$ & & & \\
\hline$-5.000715 E-03$ & $6.837076 \mathrm{E}-04$ & & & & $3 / 16 / 996: 19: 43$ & & & \\
\hline$-5.000710 \mathrm{E}-03$ & $6.837094 \mathrm{E}-04$ & & & & $3 / 16 / 996: 19: 45$ & & & \\
\hline $5.000202 \mathrm{E}-03$ & $-6.988236 \mathrm{E}-04$ & & & & 3/16/99 6:19:48 & & & \\
\hline $5.000204 \mathrm{E}-03$ & $-6.988128 \mathrm{E}-04$ & & & & $3 / 16 / 996: 19: 51$ & & & \\
\hline$-5.000710 \mathrm{E}-03$ & $6.837033 \mathrm{E}-04$ & & & & $3 / 16 / 996: 19: 54$ & & & \\
\hline$-5.000711 \mathrm{E}-03$ & $6.837030 \mathrm{E}-04$ & & & & $3 / 16 / 996: 19: 57$ & & & \\
\hline $5.000199 E-03$ & $-6.988108 E-04$ & & & & $3 / 16 / 996: 20: 00$ & & & \\
\hline $5.000202 E-03$ & $-6.988315 \mathrm{E}-04$ & & & & $3 / 16 / 996: 20: 02$ & & & \\
\hline$-5.000712 \mathrm{E}-03$ & $6.837103 \mathrm{E}-04$ & & & & 3/16/99 6:20:05 & & & \\
\hline$-5.000706 \mathrm{E}-03$ & $6.837059 \mathrm{E}-04$ & & & & $3 / 16 / 996: 20: 08$ & & & \\
\hline $5.000202 E-03$ & $-6.988230 \mathrm{E}-04$ & & & & $3 / 16 / 996: 20: 11$ & & & \\
\hline $5.000455 \mathrm{E}-03$ & $6.912653 \mathrm{E}-04$ & 0.1382405 & $1.178252 \mathrm{E}-06$ & $3.456636 \mathrm{E}-06$ & $3 / 16 / 996: 20: 15$ & 4867.324016 & 4.786034461 & \\
\hline & & & & & & & & \\
\hline & & & & & & & & \\
\hline
\end{tabular}


Copper2.xls

\begin{tabular}{|c|c|c|c|c|c|c|c|c|}
\hline \multirow{2}{*}{\begin{tabular}{|l|} 
Current $(A)$ \\
Sample ID= Cu\#2 \\
\end{tabular}} & Voltage (V) & Res.(ohms) & \multirow{2}{*}{$\frac{\text { Std. Dev. Of } \Omega}{\text { rget }=0}$} & \multirow[t]{2}{*}{ Power (W) } & \multirow[t]{2}{*}{ Date/time } & \multirow[t]{2}{*}{ Cernox (ohms) } & \multicolumn{2}{|c|}{ Cernox (K) 4th order poly } \\
\hline & Beam Energy $=$ & $=0 \quad$ Time on ta & & & & & & \\
\hline \multicolumn{9}{|c|}{ Pre-irradiation resistivity data at liquid helium temperature prior to testing at $1.94 \mathrm{GeV}$} \\
\hline $9.999787 \mathrm{E}-03$ & $-1.389999 \mathrm{E}-03$ & & & & $3 / 16 / 996: 21: 10$ & & & \\
\hline$-1.000001 E-02$ & $1.374765 \mathrm{E}-03$ & & & & $3 / 16 / 996: 21: 13$ & & & \\
\hline$-1.000001 \mathrm{E}-02$ & $1.374772 \mathrm{E}-03$ & & & & $3 / 16 / 996: 21: 16$ & & & \\
\hline 9.999796E-03 & $-1.390011 E-03$ & & & & $3 / 16 / 996: 21: 18$ & & & \\
\hline 9.999794E-03 & $-1.390003 E-03$ & & & & $3 / 16 / 996: 21: 21$ & & & \\
\hline$-1.000001 E-02$ & $1.374759 \mathrm{E}-03$ & & & & $3 / 16 / 996: 21: 24$ & & & \\
\hline$-1.000001 E-02$ & 1.374765E-03 & & & & $3 / 16 / 996: 21: 27$ & & & \\
\hline $9.999800 \mathrm{E}-03$ & $-1.389997 \mathrm{E}-03$ & & & & $3 / 16 / 996: 21: 30$ & & & \\
\hline 9.999794E-03 & $-1.389987 \mathrm{E}-03$ & & & & $3 / 16 / 996: 21: 33$ & & & \\
\hline$-1.000001 E-02$ & $1.374778 \mathrm{E}-03$ & & & & $3 / 16 / 996: 21: 35$ & & & \\
\hline$-1.000000 E-02$ & $1.374788 \mathrm{E}-03$ & & & & $3 / 16 / 996: 21: 38$ & & & \\
\hline $9.999797 \mathrm{E}-03$ & $-1.389983 \mathrm{E}-03$ & & & & $3 / 16 / 996: 21: 41$ & & & \\
\hline $9.999790 \mathrm{E}-03$ & $-1.389976 \mathrm{E}-03$ & & & & $3 / 16 / 996: 21: 44$ & & & \\
\hline$-1.000002 E-02$ & 1.374807E-03 & & & & $3 / 16 / 996: 21: 47$ & & & \\
\hline$-1.000001 E-02$ & 1.374793E-03 & & & & $3 / 16 / 996: 21: 50$ & & & \\
\hline 9.999798E-03 & $-1.389984 E-03$ & & & & $3 / 16 / 996: 21: 52$ & & & \\
\hline 9.999794E-03 & $-1.389966 \mathrm{E}-03$ & & & & $3 / 16 / 996: 21: 55$ & & & \\
\hline$-1.000001 E-02$ & 1.374812E-03 & & & & $3 / 16 / 996: 21: 58$ & & & \\
\hline$-1.000001 E-02$ & $1.374802 \mathrm{E}-03$ & & & & $3 / 16 / 996: 22: 01$ & & & \\
\hline 9.999799E-03 & $-1.389968 \mathrm{E}-03$ & & & & $3 / 16 / 996: 22: 04$ & & & \\
\hline $9.999789 E-03$ & $-1.389979 \mathrm{E}-03$ & & & & $3 / 16 / 996: 22: 07$ & & & \\
\hline$-1.000002 E-02$ & 1.374800E-03 & & & & $3 / 16 / 996: 22: 09$ & & & \\
\hline$-1.000002 E-02$ & 1.374805E-03 & & & & $3 / 16 / 996: 22: 12$ & & & \\
\hline 9.999797E-03 & $-1.389959 \mathrm{E}-03$ & & & & $3 / 16 / 996: 22: 15$ & & & \\
\hline 9.999793E-03 & $-1.389979 \mathrm{E}-03$ & & & & $3 / 16 / 996: 22: 18$ & & & \\
\hline$-1.000002 E-02$ & 1.374816E-03 & & & & $3 / 16 / 996: 22: 21$ & & & \\
\hline$-1.000002 E-02$ & $1.374822 \mathrm{E}-03$ & & & & $3 / 16 / 996: 22: 24$ & & & \\
\hline $9.999796 \mathrm{E}-03$ & $-1.389957 E-03$ & & & & $3 / 16 / 996: 22: 26$ & & & \\
\hline $9.999791 \mathrm{E}-03$ & $-1.389954 E-03$ & & & & $3 / 16 / 996: 22: 29$ & & & \\
\hline$-1.000002 E-02$ & 1.374833E-03 & & & & $3 / 16 / 996: 22: 32$ & & & \\
\hline$-1.000001 E-02$ & 1.374831E-03 & & & & $3 / 16 / 996: 22: 35$ & & & \\
\hline $9.999792 \mathrm{E}-03$ & $-1.389950 \mathrm{E}-03$ & & & & $3 / 16 / 996: 22: 38$ & & & \\
\hline 9.999784E-03 & $-1.389963 E-03$ & & & & $3 / 16 / 996: 22: 40$ & & & \\
\hline$-1.000002 E-02$ & 1.374826E-03 & & & & $3 / 16 / 996: 22: 43$ & & & \\
\hline$-1.000002 E-02$ & 1.374812E-03 & & & & $3 / 16 / 996: 22: 46$ & & & \\
\hline 9.999789E-03 & $-1.389943 E-03$ & & & & $3 / 16 / 996: 22: 49$ & & & \\
\hline $9.999781 \mathrm{E}-03$ & $-1.389968 E-03$ & & & & $3 / 16 / 996: 22: 52$ & & & \\
\hline$-1.000002 E-02$ & $1.374815 \mathrm{E}-03$ & & & & $3 / 16 / 996: 22: 55$ & & & \\
\hline$-1.000002 E-02$ & 1.374820E-03 & & & & $3 / 16 / 996: 22: 57$ & & & \\
\hline $9.999787 \mathrm{E}-03$ & $-1.389961 \mathrm{E}-03$ & & & & $3 / 16 / 996: 23: 00$ & & & \\
\hline 9.999903E-03 & $1.382388 \mathrm{E}-03$ & 0.1382401 & 5.158274E-07 & $1.382377 \mathrm{E}-05$ & $3 / 16 / 996: 23: 04$ & 4867.497871 & 4.785938117 & \\
\hline & & & & & & & & \\
\hline
\end{tabular}


Copper2.xis

\begin{tabular}{|c|c|c|c|c|c|c|c|c|}
\hline \begin{tabular}{|l|} 
Current $(A)$ \\
Sample ID $=$ Cu\#2 \\
\end{tabular} & \begin{tabular}{|l|} 
Voltage $(\mathrm{V})$ \\
Beam Energy
\end{tabular} & Res.(ohms) & $\begin{array}{l}\text { Std. Dev. Of } \Omega \\
\text { arget }=0\end{array}$ & Power (W) & Date/time & Cernox (ohms) & \multicolumn{2}{|c|}{ Cernox $(K)$ 4th order poly. } \\
\hline $1.499905 \mathrm{E}-02$ & $-2.081237 \mathrm{E}-03$ & & & & $3 / 16 / 996: 23: 57$ & & & \\
\hline$-1.500318 \mathrm{E}-02$ & $2.066247 \mathrm{E}-03$ & & & & $3 / 16 / 996: 24: 03$ & & & \\
\hline $1.499900 \mathrm{E}-02$ & $-2.081232 E-03$ & & & & $3 / 16 / 996: 24: 06$ & & & \\
\hline $1.499901 \mathrm{E}-02$ & $-2.081233 E-03$ & & & & $3 / 16 / 996: 24: 09$ & & & \\
\hline$-1.500317 \mathrm{E}-02$ & $2.066241 \mathrm{E}-03$ & & & & $3 / 16 / 99$ 6:24:12 & & & \\
\hline $1.499905 \mathrm{E}-02$ & $-2.081244 \mathrm{E}-03$ & & & & $3 / 16 / 996: 24: 20$ & & & \\
\hline$-1.500306 \mathrm{E}-02$ & $2.066228 \mathrm{E}-03$ & & & & $3 / 16 / 996: 24: 23$ & & & \\
\hline$-1.500306 \mathrm{E}-02$ & $2.066229 \mathrm{E}-03$ & & & & $3 / 16 / 996: 24: 26$ & & & \\
\hline $1.499907 \mathrm{E}-02$ & $-2.081255 E-03$ & & & & $3 / 16 / 996: 24: 28$ & & & \\
\hline $1.499906 \mathrm{E}-02$ & $-2.081229 \mathrm{E}-03$ & & & & $3 / 16 / 99$ 6:24:31 & & & \\
\hline$-1.500314 \mathrm{E}-02$ & $2.066218 \mathrm{E}-03$ & & & & $3 / 16 / 996: 24: 34$ & & & \\
\hline$-1.500309 E-02$ & $2.066262 \mathrm{E}-03$ & & & & $3 / 16 / 99$ 6:24:37 & & & \\
\hline 1.499903E-02 & $-2.081240 \mathrm{E}-03$ & & & & $3 / 16 / 996: 24: 54$ & & & \\
\hline$-1.500316 \mathrm{E}-02$ & $2.066241 \mathrm{E}-03$ & & & & $3 / 16 / 996: 24: 57$ & & & \\
\hline$-1.500311 \mathrm{E}-02$ & $2.066249 \mathrm{E}-03$ & & & & $3 / 16 / 99$ 6:25:00 & & & \\
\hline $1.499905 \mathrm{E}-02$ & $-2.081244 \mathrm{E}-03$ & & & & $3 / 16 / 996: 25: 02$ & & & \\
\hline 1.499905E-02 & $-2.081246 \mathrm{E}-03$ & & & & $3 / 16 / 996: 25: 05$ & & & \\
\hline$-1.500314 \mathrm{E}-02$ & $2.066241 \mathrm{E}-03$ & & & & $3 / 16 / 99$ 6:25:08 & & & \\
\hline$-1.500310 \mathrm{E}-02$ & $2.066244 \mathrm{E}-03$ & & & & $3 / 16 / 996: 25: 11$ & & & \\
\hline $1.499902 \mathrm{E}-02$ & $-2.081228 \mathrm{E}-03$ & & & & 3/16/99 6:25:14 & & & \\
\hline $1.499907 \mathrm{E}-02$ & $-2.081243 E-03$ & & & & $3 / 16 / 996: 25: 17$ & & & \\
\hline$-1.500315 \mathrm{E}-02$ & $2.066270 \mathrm{E}-03$ & & & & $3 / 16 / 996: 25: 20$ & & & \\
\hline$-1.500318 \mathrm{E}-02$ & $2.066256 \mathrm{E}-03$ & & & & $3 / 16 / 996: 25: 22$ & & & \\
\hline 1.499906E-02 & $-2.081235 E-03$ & & & & $3 / 16 / 996: 25: 25$ & & & \\
\hline 1.499907E-02 & $-2.081241 E-03$ & & & & $3 / 16 / 996: 25: 28$ & & & \\
\hline$-1.500318 \mathrm{E}-02$ & $2.066251 \mathrm{E}-03$ & & & & $3 / 16 / 996: 25: 31$ & & & \\
\hline
\end{tabular}


Copper2.xls

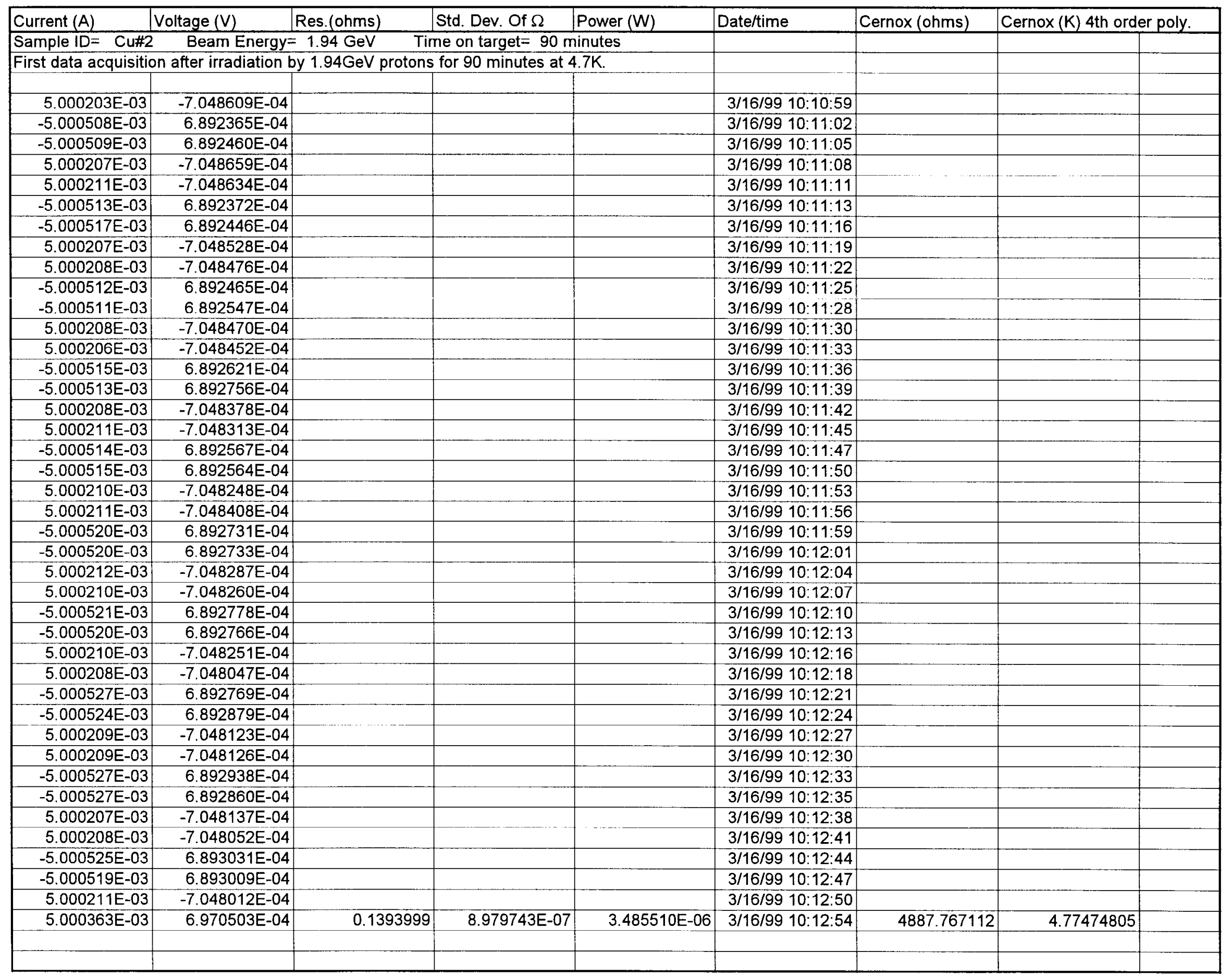


Copper2.xis

\begin{tabular}{|c|c|c|c|c|c|c|c|c|}
\hline \multirow{2}{*}{\begin{tabular}{|l|} 
Current $(A)$ \\
Sample ID $=$ Cu\#2 \\
First data acquisitic
\end{tabular}} & Voltage $(\mathrm{V})$ & Res.(ohms) & & & \multirow[t]{2}{*}{ Date/time } & \multirow[t]{2}{*}{ Cernox (ohms) } & \multicolumn{2}{|c|}{ Cernox $(\mathrm{K})$ 4th order poly. } \\
\hline & \multicolumn{2}{|c|}{ Beam Energy $=1.94 \mathrm{GeV}$} & $\begin{array}{l}\text { Std. Dev. Of } \Omega \\
\text { Time on target }=90 \text { minutes }\end{array}$ & & & & & \\
\hline & & & & & & & & \\
\hline $9.999855 \mathrm{E}-03$ & $-1.401701 \mathrm{E}-03$ & & & & 3/16/99 10:13:47 & & & \\
\hline$-9.999875 \mathrm{E}-03$ & $1.386225 \mathrm{E}-03$ & & & & $3 / 16 / 99$ 10:13:52 & & & \\
\hline $9.999860 \mathrm{E}-03$ & $-1.401703 \mathrm{E}-03$ & & & & $3 / 16 / 99$ 10:13:55 & & & \\
\hline $9.999854 \mathrm{E}-03$ & $-1.401697 \mathrm{E}-03$ & & & & $3 / 16 / 99$ 10:13:58 & & & \\
\hline $9.999854 \mathrm{E}-03$ & $-1.401695 \mathrm{E}-03$ & & & & $3 / 16 / 99$ 10:14:09 & & & \\
\hline$-9.999885 \mathrm{E}-03$ & $1.386235 \mathrm{E}-03$ & & & & $3 / 16 / 99$ 10:14:12 & & & \\
\hline$-9.999879 \mathrm{E}-03$ & $1.386230 \mathrm{E}-03$ & & & & $3 / 16 / 99$ 10:14:15 & & & \\
\hline 9.999859E-03 & $-1.401680 \mathrm{E}-03$ & & & & $3 / 16 / 99$ 10:14:18 & & & \\
\hline $9.999852 E-03$ & $-1.401697 \mathrm{E}-03$ & & & & $3 / 16 / 99$ 10:14:20 & & & \\
\hline$-9.999884 E-03$ & $1.386228 \mathrm{E}-03$ & & & & $3 / 16 / 99$ 10:14:23 & & & \\
\hline$-9.999884 E-03$ & $1.386237 \mathrm{E}-03$ & & & & 3/16/99 10:14:26 & & & \\
\hline $9.999847 \mathrm{E}-03$ & $-1.401680 \mathrm{E}-03$ & & & & $3 / 16 / 99$ 10:14:43 & & & \\
\hline$-9.999887 \mathrm{E}-03$ & $1.386246 \mathrm{E}-03$ & & & & $3 / 16 / 99$ 10:14:46 & & & \\
\hline$-9.999882 \mathrm{E}-03$ & $1.386244 \mathrm{E}-03$ & & & & $3 / 16 / 99 \quad 10: 14: 49$ & & & \\
\hline $9.999847 \mathrm{E}-03$ & $-1.401676 \mathrm{E}-03$ & & & & $3 / 16 / 99$ 10:14:52 & & & \\
\hline $9.999850 \mathrm{E}-03$ & $-1.401686 \mathrm{E}-03$ & & & & $3 / 16 / 99$ 10:14:54 & & & \\
\hline$-9.999885 E-03$ & $1.386235 \mathrm{E}-03$ & & & & 3/16/99 10:14:57 & & & \\
\hline$-9.999881 \mathrm{E}-03$ & $1.386248 \mathrm{E}-03$ & & & & $3 / 16 / 99$ 10:15:00 & & & \\
\hline $9.999853 \mathrm{E}-03$ & $-1.401678 \mathrm{E}-03$ & & & & $3 / 16 / 99$ 10:15:03 & & & \\
\hline 9.999847E-03 & $-1.401671 \mathrm{E}-03$ & & & & $3 / 16 / 99$ 10:15:06 & & & \\
\hline$-9.999885 \mathrm{E}-03$ & $1.386236 \mathrm{E}-03$ & & & & $3 / 16 / 99$ 10:15:09 & & & \\
\hline$-9.999883 E-03$ & $1.386243 E-03$ & & & & 3/16/99 10:15:11 & & & \\
\hline $9.999858 \mathrm{E}-03$ & $-1.401672 \mathrm{E}-03$ & & & & $3 / 16 / 99$ 10:15:14 & & & \\
\hline 9.999854E-03 & $-1.401668 \mathrm{E}-03$ & & & & $3 / 16 / 99$ 10:15:17 & & & \\
\hline$-9.999883 E-03$ & $1.386249 \mathrm{E}-03$ & & & & 3/16/99 10:15:20 & & & \\
\hline
\end{tabular}


Copper2.xis

\begin{tabular}{|c|c|c|c|c|c|c|c|c|}
\hline \multirow{3}{*}{$\frac{\text { Current }(A)}{\text { Sample ID }=\text { Cu\#2 }}$} & Voltage $(\mathrm{V})$ & Res.(ohms) & & & \multirow[t]{2}{*}{ Date/time } & \multirow[t]{2}{*}{ Cernox (ohms) } & \multicolumn{2}{|c|}{ Cernox (K) 4th order poly. } \\
\hline & \multicolumn{2}{|c|}{ Beam Energy $=1.94 \mathrm{GeV}$} & \begin{tabular}{l|l} 
Std. Dev. Of $\Omega$ & Power $(\mathrm{W})$ \\
Time on target $=90$ minutes
\end{tabular} & & & & & \\
\hline & on after irradiation b & by $1.94 \mathrm{GeV}$ protons & \multicolumn{2}{|c|}{ ns for 90 minutes at $4.7 \mathrm{~K}$} & & & & \\
\hline 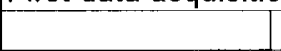 & & & & & & & & \\
\hline $1.499840 \mathrm{E}-02$ & $-2.098758 E-03$ & & & & $3 / 16 / 99$ 10:16:29 & & & \\
\hline$-1.500086 \mathrm{E}-02$ & $2.083082 \mathrm{E}-03$ & & & & $3 / 16 / 99$ 10:16:31 & & & \\
\hline$-1.500086 \mathrm{E}-02$ & $2.083088 \mathrm{E}-03$ & & & & $3 / 16 / 99$ 10:16:34 & & & \\
\hline $1.499845 E-02$ & $-2.098763 \mathrm{E}-03$ & & & & $3 / 16 / 99$ 10:16:37 & & & \\
\hline 1.499849E-02 & $-2.098758 \mathrm{E}-03$ & & & & $3 / 16 / 99$ 10:16:40 & & & \\
\hline$-1.500083 E-02$ & 2.083104E-03 & & & & 3/16/99 10:16:43 & & & \\
\hline$-1.500081 \mathrm{E}-02$ & $2.083086 E-03$ & & & & $3 / 16 / 9910: 16: 46$ & & & \\
\hline $1.499856 \mathrm{E}-02$ & $-2.098768 \mathrm{E}-03$ & & & & $3 / 16 / 99$ 10:16:48 & & & \\
\hline $1.499856 \mathrm{E}-02$ & $-2.098764 \mathrm{E}-03$ & & & & $3 / 16 / 99$ 10:16:51 & & & \\
\hline$-1.500077 \mathrm{E}-02$ & 2.083104E-03 & & & & $3 / 16 / 99$ 10:16:54 & & & \\
\hline$-1.500076 \mathrm{E}-02$ & $2.083116 \mathrm{E}-03$ & & & & $3 / 16 / 99$ 10:16:57 & & & \\
\hline $1.499857 \mathrm{E}-02$ & $-2.098767 \mathrm{E}-03$ & & & & $3 / 16 / 99$ 10:17:00 & & & \\
\hline $1.499858 \mathrm{E}-02$ & $-2.098764 E-03$ & & & & $3 / 16 / 99$ 10:17:02 & & & \\
\hline$-1.500082 E-02$ & $2.083102 E-03$ & & & & $3 / 16 / 99$ 10:17:05 & & & \\
\hline$-1.500080 \mathrm{E}-02$ & $2.083104 \mathrm{E}-03$ & & & & $3 / 16 / 99$ 10:17:08 & & & \\
\hline $1.499852 E-02$ & $-2.098748 \mathrm{E}-03$ & & & & $3 / 16 / 99$ 10:17:11 & & & \\
\hline 1.499850E-02 & $-2.098768 \mathrm{E}-03$ & & & & $3 / 16 / 99$ 10:17:14 & & & \\
\hline$-1.500078 \mathrm{E}-02$ & $2.083096 \mathrm{E}-03$ & & & & $3 / 16 / 99$ 10:17:17 & & & \\
\hline$-1.500081 \mathrm{E}-02$ & 2.083117E-03 & & & & $3 / 16 / 99$ 10:17:19 & & & \\
\hline 1.499849E-02 & $-2.098728 \mathrm{E}-03$ & & & & $3 / 16 / 99$ 10:17:22 & & & \\
\hline $1.499853 \mathrm{E}-02$ & $-2.098752 E-03$ & & & & $3 / 16 / 99$ 10:17:25 & & & \\
\hline$-1.500079 \mathrm{E}-02$ & $2.083113 E-03$ & & & & $3 / 16 / 99 \quad 10: 17: 28$ & & & \\
\hline$-1.500080 \mathrm{E}-02$ & $2.083117 E-03$ & & & & $3 / 16 / 99$ 10:17:31 & & & \\
\hline $1.499852 \mathrm{E}-02$ & $-2.098744 \mathrm{E}-03$ & & & & $3 / 16 / 99$ 10:17:34 & & & \\
\hline 1.499854E-02 & $-2.098738 \mathrm{E}-03$ & & & & $3 / 16 / 99$ 10:17:36 & & & \\
\hline$-1.500082 E-02$ & $2.083102 E-03$ & & & & $3 / 16 / 99 \quad 10: 17: 39$ & & & \\
\hline$-1.500081 E-02$ & $2.083090 E-03$ & & & & $3 / 16 / 9910: 17: 42$ & & & \\
\hline 1.499852E-02 & $-2.098742 E-03$ & & & & $3 / 16 / 99$ 10:17:45 & & & \\
\hline 1.499850E-02 & $-2.098744 E-03$ & & & & $3 / 16 / 99$ 10:17:48 & & & \\
\hline$-1.500085 E-02$ & $2.083099 \mathrm{E}-03$ & & & & $3 / 16 / 99$ 10:17:51 & & & \\
\hline$-1.500082 E-02$ & $2.083115 E-03$ & & & & $3 / 16 / 99$ 10:17:53 & & & \\
\hline 1.499857E-02 & $-2.098760 \mathrm{E}-03$ & & & & $3 / 16 / 99$ 10:17:56 & & & \\
\hline $1.499857 \mathrm{E}-02$ & $-2.098750 \mathrm{E}-03$ & & & & $3 / 16 / 99$ 10:17:59 & & & \\
\hline$-1.500083 E-02$ & $2.083124 \mathrm{E}-03$ & & & & $3 / 16 / 9910: 18: 02$ & & & \\
\hline$-1.500085 E-02$ & $2.083123 E-03$ & & & & 3/16/99 10:18:05 & & & \\
\hline 1.499853E-02 & $-2.098748 E-03$ & & & & $3 / 16 / 99$ 10:18:08 & & & \\
\hline 1.499855E-02 & $-2.098741 \mathrm{E}-03$ & & & & $3 / 16 / 99$ 10:18:10 & & & \\
\hline$-1.500088 \mathrm{E}-02$ & $2.083133 E-03$ & & & & $3 / 16 / 99$ 10:18:13 & & & \\
\hline$-1.500091 \mathrm{E}-02$ & $2.083118 E-03$ & & & & $3 / 16 / 99$ 10:18:16 & & & \\
\hline 1.499853E-02 & $-2.098742 E-03$ & & & & $3 / 16 / 99$ 10:18:19 & & & \\
\hline 1.499967E-02 & $2.090929 \mathrm{E}-03$ & 0.1393983 & 4.126809E-07 & 3.136336E-05 & $3 / 16 / 99$ 10:18:23 & 4882.698441 & 4.77753845 & \\
\hline & & & & & & & & \\
\hline
\end{tabular}


Copper2.xls

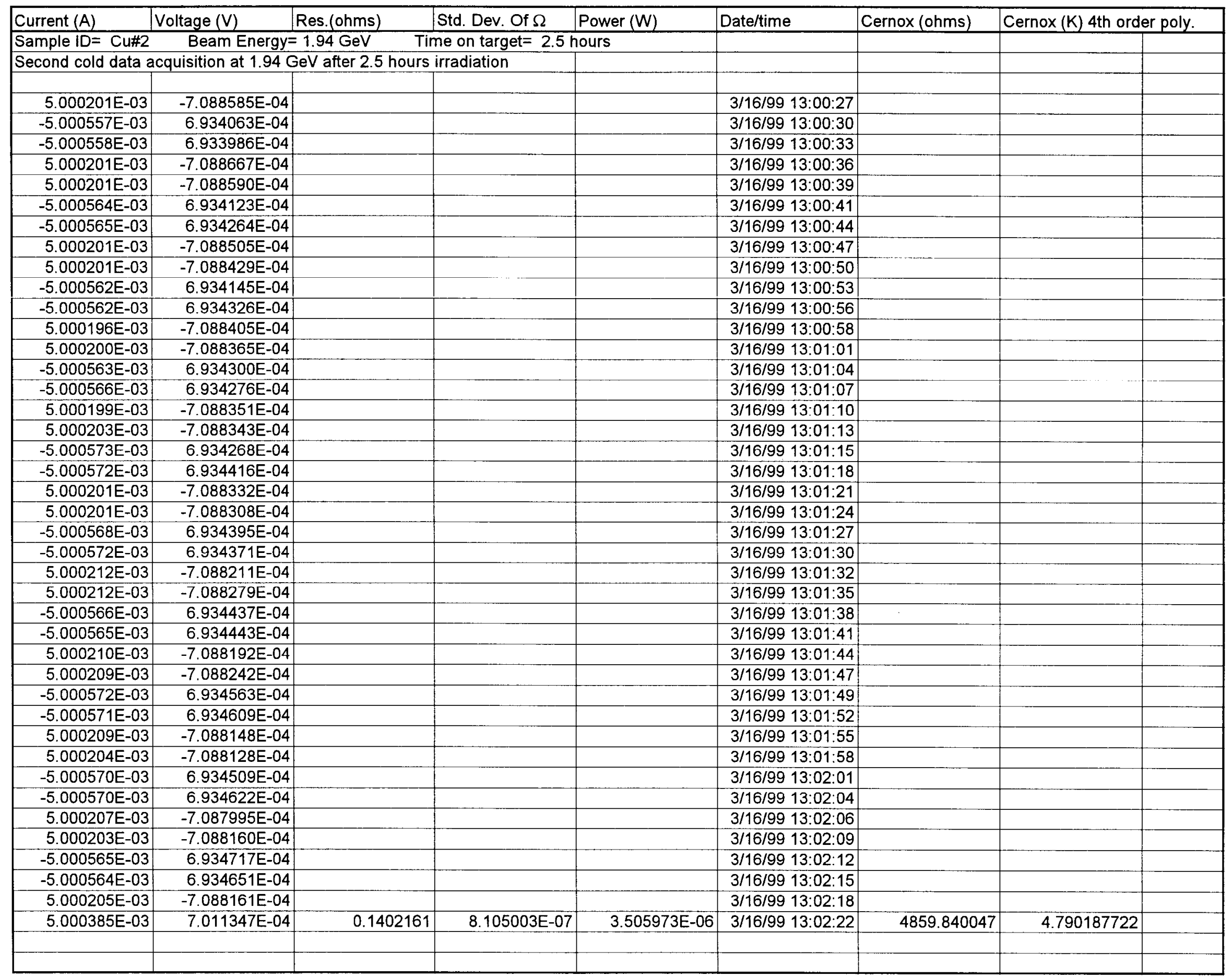


Copper2.xls

\begin{tabular}{|c|c|c|c|c|c|c|c|c|}
\hline Current $(A)$ & Voltage (V) & Res.(ohms) & Std. Dev. Of $\Omega$ & Power (W) & Date/time & Cernox (ohms) & \multicolumn{2}{|c|}{ Cernox $(K) 4$ th order poly. } \\
\hline Sample ID= Cu\#2 & \multicolumn{2}{|c|}{ Beam Energy $=1.94 \mathrm{GeV}$} & \multicolumn{2}{|c|}{ ime on target $=2.5$ hours } & & & & \\
\hline \multicolumn{9}{|c|}{ Second cold data acquisition at $1.94 \mathrm{GeV}$ after 2.5 hours irradiation } \\
\hline 9.999827E-03 & $-1.409817 E-03$ & & & & 3/16/99 13:03:06 & & & \\
\hline$-9.999918 \mathrm{E}-03$ & 1.394470E-03 & & & & 3/16/99 13:03:09 & & & \\
\hline$-9.999917 E-03$ & $1.394478 \mathrm{E}-03$ & & & & $3 / 16 / 9913: 03: 12$ & & & \\
\hline 9.999831E-03 & $-1.409810 E-03$ & & & & 3/16/99 13:03:15 & & & \\
\hline 9.999829E-03 & $-1.409798 \mathrm{E}-03$ & & & & $3 / 16 / 9913: 03: 17$ & & & \\
\hline$-9.999915 E-03$ & 1.394457E-03 & & & & $3 / 16 / 99$ 13:03:20 & & & \\
\hline$-9.999909 \mathrm{E}-03$ & 1.394465E-03 & & & & 3/16/99 13:03:23 & & & \\
\hline 9.999834E-03 & $-1.409803 \mathrm{E}-03$ & & & & $3 / 16 / 9913: 03: 26$ & & & \\
\hline $9.999835 \mathrm{E}-03$ & $-1.409806 \mathrm{E}-03$ & & & & $3 / 16 / 9913: 03: 29$ & & & \\
\hline$-9.999914 \mathrm{E}-03$ & 1.394473E-03 & & & & $3 / 16 / 9913: 03: 32$ & & & \\
\hline$-9.999909 E-03$ & 1.394451E-03 & & & & $3 / 16 / 9913: 03: 34$ & & & \\
\hline 9.999833E-03 & $-1.409805 E-03$ & & & & $3 / 16 / 9913: 03: 37$ & & & \\
\hline $9.999832 E-03$ & $-1.409814 \mathrm{E}-03$ & & & & $3 / 16 / 9913: 03: 40$ & & & \\
\hline$-9.999916 \mathrm{E}-03$ & $1.394466 \mathrm{E}-03$ & & & & $3 / 16 / 9913: 03: 43$ & & & \\
\hline$-9.999913 \mathrm{E}-03$ & 1.394475E-03 & & & & $3 / 16 / 9913: 03: 46$ & & & \\
\hline 9.999833E-03 & $-1.409805 E-03$ & & & & $3 / 16 / 99$ 13:03:49 & & & \\
\hline $9.999827 \mathrm{E}-03$ & $-1.409798 E-03$ & & & & $3 / 16 / 9913: 03: 51$ & & & \\
\hline$-9.999922 \mathrm{E}-03$ & 1.394474E-03 & & & & $3 / 16 / 9913: 03: 54$ & & & \\
\hline$-9.999922 \mathrm{E}-03$ & 1.394469E-03 & & & & $3 / 16 / 9913: 03: 57$ & & & \\
\hline $9.999831 \mathrm{E}-03$ & $-1.409791 \mathrm{E}-03$ & & & & $3 / 16 / 9913: 04: 00$ & & & \\
\hline $9.999827 \mathrm{E}-03$ & $-1.409801 \mathrm{E}-03$ & & & & $3 / 16 / 9913: 04: 03$ & & & \\
\hline$-9.999921 E-03$ & 1.394476E-03 & & & & $3 / 16 / 9913: 04: 05$ & & & \\
\hline$-9.999912 \mathrm{E}-03$ & $1.394469 \mathrm{E}-03$ & & & & $3 / 16 / 9913: 04: 08$ & & & \\
\hline $9.999836 \mathrm{E}-03$ & $-1.409798 E-03$ & & & & $3 / 16 / 9913: 04: 11$ & & & \\
\hline $9.999832 \mathrm{E}-03$ & $-1.409809 \mathrm{E}-03$ & & & & $3 / 16 / 9913: 04: 14$ & & & \\
\hline$-9.999921 E-03$ & $1.394471 \mathrm{E}-03$ & & & & $3 / 16 / 9913: 04: 17$ & & & \\
\hline$-9.999918 \mathrm{E}-03$ & $1.394474 \mathrm{E}-03$ & & & & $3 / 16 / 99$ 13:04:20 & & & \\
\hline 9.999835E-03 & $-1.409790 \mathrm{E}-03$ & & & & $3 / 16 / 9913: 04: 22$ & & & \\
\hline 9.999833E-03 & $-1.409798 E-03$ & & & & $3 / 16 / 9913: 04: 25$ & & & \\
\hline$-9.999926 \mathrm{E}-03$ & $1.394495 \mathrm{E}-03$ & & & & $3 / 16 / 99$ 13:04:28 & & & \\
\hline$-9.999922 \mathrm{E}-03$ & 1.394477E-03 & & & & $3 / 16 / 9913: 04: 31$ & & & \\
\hline 9.999836E-03 & $-1.409784 \mathrm{E}-03$ & & & & $3 / 16 / 9913: 04: 34$ & & & \\
\hline $9.999829 \mathrm{E}-03$ & $-1.409790 \mathrm{E}-03$ & & & & $3 / 16 / 9913: 04: 37$ & & & \\
\hline$-9.999926 \mathrm{E}-03$ & $1.394478 \mathrm{E}-03$ & & & & $3 / 16 / 99$ 13:04:39 & & & \\
\hline$-9.999925 \mathrm{E}-03$ & $1.394488 \mathrm{E}-03$ & & & & $3 / 16 / 99$ 13:04:42 & & & \\
\hline $9.999835 \mathrm{E}-03$ & $-1.409784 \mathrm{E}-03$ & & & & $3 / 16 / 9913: 04: 45$ & & & \\
\hline $9.999833 \mathrm{E}-03$ & $-1.409790 \mathrm{E}-03$ & & & & $3 / 16 / 99$ 13:04:48 & & & \\
\hline$-9.999923 E-03$ & 1.394475E-03 & & & & $3 / 16 / 99$ 13:04:51 & & & \\
\hline$-9.999917 \mathrm{E}-03$ & $1.394480 \mathrm{E}-03$ & & & & $3 / 16 / 9913: 04: 54$ & & & \\
\hline $9.999836 \mathrm{E}-03$ & $-1.409804 \mathrm{E}-03$ & & & & $3 / 16 / 99$ 13:04:56 & & & \\
\hline $9.999875 \mathrm{E}-03$ & 1.402136E-03 & 0.1402154 & 5.262513E-07 & 1.402125E-05 & $3 / 16 / 9913: 05: 01$ & 4860.54928 & 4.789793634 & \\
\hline & & & & & & & & \\
\hline
\end{tabular}


Copper2.xls

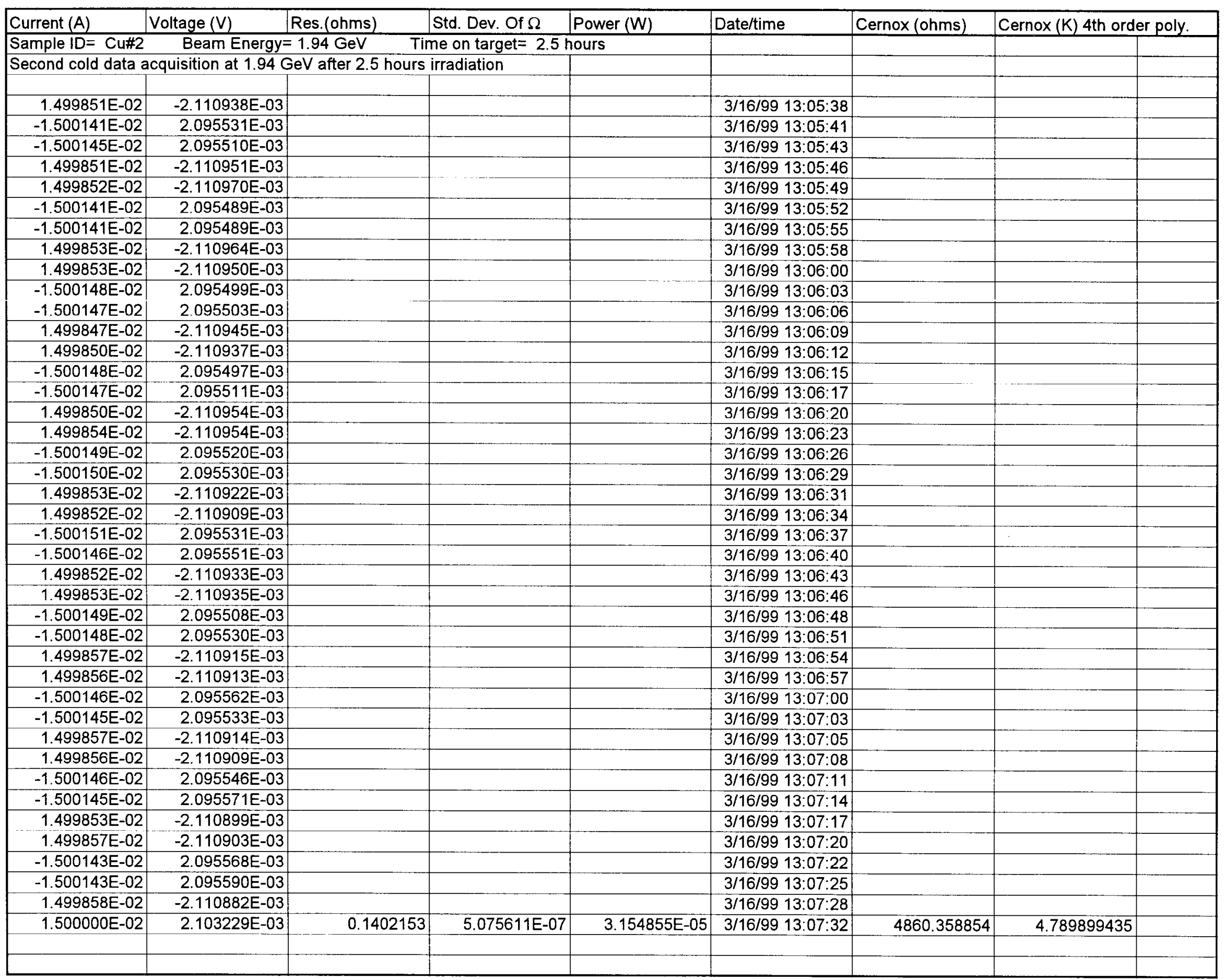


Copper2.xls

\begin{tabular}{|c|c|c|c|c|c|c|c|c|}
\hline Current $(A)$ & Voltage (V) & Res.(ohms) & Std. Dev. Of $\Omega$ & Power (W) & Date/time & Cernox (ohms) & Cernox (K) 4th ord & er poly. \\
\hline Sample ID= Cu\#2 & Beam Energy= & $=1.94 \mathrm{GeV}$ & me on target $=$ appr & oximately 13 minute & & & & \\
\hline Run aborted after 1 & 13 minutes at 1.94 & $\mathrm{GeV}$ due to upset $\mathrm{cc}$ & onditions in LHe su & pply flow rate & & & & \\
\hline & & & & & & & & \\
\hline $5.000193 \mathrm{E}-03$ & $-7.088767 E-04$ & & & & $3 / 16 / 9914: 52: 35$ & & & \\
\hline$-5.000528 \mathrm{E}-03$ & 6.914972E-04 & & & & $3 / 16 / 9914: 52: 38$ & & & \\
\hline$-5.000530 \mathrm{E}-03$ & 6.915132E-04 & & & & $3 / 16 / 9914: 52: 40$ & & & \\
\hline $5.000194 \mathrm{E}-03$ & $-7.088857 \mathrm{E}-04$ & & & & $3 / 16 / 9914: 52: 43$ & & & \\
\hline $5.000193 \mathrm{E}-03$ & $-7.088709 \mathrm{E}-04$ & & & & $3 / 16 / 9914: 52: 46$ & & & \\
\hline$-5.000531 \mathrm{E}-03$ & 6.915075E-04 & & & & $3 / 16 / 9914: 52: 49$ & & & \\
\hline$-5.000529 E-03$ & $6.915065 E-04$ & & & & $3 / 16 / 9914: 52: 52$ & & & \\
\hline 5.000195E-03 & $-7.088693 \mathrm{E}-04$ & & & & $3 / 16 / 9914: 52: 55$ & & & \\
\hline $5.000198 \mathrm{E}-03$ & $-7.088630 \mathrm{E}-04$ & & & & $3 / 16 / 9914: 52: 57$ & & & \\
\hline$-5.000532 E-03$ & $6.915138 \mathrm{E}-04$ & & & & $3 / 16 / 9914: 53: 00$ & & & \\
\hline$-5.000530 \mathrm{E}-03$ & $6.915014 \mathrm{E}-04$ & & & & $3 / 16 / 9914: 53: 03$ & & & \\
\hline 5.000203E-03 & $-7.088475 \mathrm{E}-04$ & & & & $3 / 16 / 9914: 53: 06$ & & & \\
\hline $5.000202 \mathrm{E}-03$ & $-7.088602 \mathrm{E}-04$ & & & & 3/16/99 14:53:09 & & & \\
\hline$-5.000533 E-03$ & $6.915036 \mathrm{E}-04$ & & & & $3 / 16 / 9914: 53: 11$ & & & \\
\hline$-5.000530 \mathrm{E}-03$ & $6.915073 \mathrm{E}-04$ & & & & $3 / 16 / 99$ 14:53:14 & & & \\
\hline $5.000200 \mathrm{E}-03$ & $-7.088446 \mathrm{E}-04$ & & & & $3 / 16 / 9914: 53: 17$ & & & \\
\hline 5.000198E-03 & $-7.088545 \mathrm{E}-04$ & & & & $3 / 16 / 9914: 53: 20$ & & & \\
\hline$-5.000536 \mathrm{E}-03$ & $6.915153 \mathrm{E}-04$ & & & & $3 / 16 / 9914: 53: 23$ & & & \\
\hline$-5.000535 E-03$ & $6.915243 \mathrm{E}-04$ & & & & $3 / 16 / 9914: 53: 26$ & & & \\
\hline $5.000197 \mathrm{E}-03$ & $-7.088295 \mathrm{E}-04$ & & & & $3 / 16 / 9914: 53: 28$ & & & \\
\hline $5.000198 \mathrm{E}-03$ & $-7.088486 \mathrm{E}-04$ & & & & $3 / 16 / 9914: 53: 31$ & & & \\
\hline$-5.000531 \mathrm{E}-03$ & $6.915289 \mathrm{E}-04$ & & & & $3 / 16 / 9914: 53: 34$ & & & \\
\hline$-5.000528 \mathrm{E}-03$ & 6.915323E-04 & & & & $3 / 16 / 9914: 53: 37$ & & & \\
\hline $5.000198 \mathrm{E}-03$ & $-7.088399 \mathrm{E}-04$ & & & - & $3 / 16 / 9914: 53: 40$ & & & \\
\hline $5.000195 \mathrm{E}-03$ & $-7.088305 \mathrm{E}-04$ & & & & $3 / 16 / 9914: 53: 43$ & & & \\
\hline$-5.000535 E-03$ & 6.915142E-04 & & & & $3 / 16 / 9914: 53: 45$ & & & \\
\hline$-5.000533 \mathrm{E}-03$ & $6.915213 \mathrm{E}-04$ & & & & $3 / 16 / 9914: 53: 48$ & & & \\
\hline $5.000193 \mathrm{E}-03$ & $-7.088331 \mathrm{E}-04$ & & & & $3 / 16 / 9914: 53: 51$ & & & \\
\hline $5.000196 \mathrm{E}-03$ & $-7.088308 \mathrm{E}-04$ & & & & $3 / 16 / 9914: 53: 54$ & & & \\
\hline$-5.000534 \mathrm{E}-03$ & $6.915171 \mathrm{E}-04$ & & & & $3 / 16 / 9914: 53: 57$ & & & \\
\hline$-5.000534 \mathrm{E}-03$ & $6.915130 \mathrm{E}-04$ & & & & $3 / 16 / 99 \quad 14: 54: 00$ & & & \\
\hline $5.000202 \mathrm{E}-03$ & $-7.088271 \mathrm{E}-04$ & & & & $3 / 16 / 9914: 54: 02$ & & & \\
\hline $5.000205 E-03$ & $-7.088264 \mathrm{E}-04$ & & & & $3 / 16 / 9914: 54: 05$ & & & \\
\hline$-5.000531 E-03$ & $6.915193 \mathrm{E}-04$ & & & & $3 / 16 / 9914: 54: 08$ & & & \\
\hline$-5.000527 \mathrm{E}-03$ & $6.915207 \mathrm{E}-04$ & & & & $3 / 16 / 9914: 54: 11$ & & & \\
\hline $5.000202 \mathrm{E}-03$ & $-7.088167 \mathrm{E}-04$ & & & & $3 / 16 / 9914: 54: 14$ & & & \\
\hline $5.000198 \mathrm{E}-03$ & $-7.088133 E-04$ & & & & $3 / 16 / 99$ 14:54:16 & & & \\
\hline$-5.000532 \mathrm{E}-03$ & $6.915138 \mathrm{E}-04$ & & & & $3 / 16 / 9914: 54: 19$ & & & \\
\hline$-5.000528 \mathrm{E}-03$ & $6.915058 \mathrm{E}-04$ & & & & $3 / 16 / 9914: 54: 22$ & & & \\
\hline $5.000202 \mathrm{E}-03$ & $-7.088038 \mathrm{E}-04$ & & & & $3 / 16 / 9914: 54: 25$ & & & \\
\hline $5.000365 \mathrm{E}-03$ & 7.001787E-04 & 0.1400255 & $2.074308 \mathrm{E}-06$ & $3.501029 \mathrm{E}-06$ & $3 / 16 / 99$ 14:54:29 & 2464.791349 & 7.280323826 & \\
\hline & & & & & & & & \\
\hline
\end{tabular}


Copper2.xls

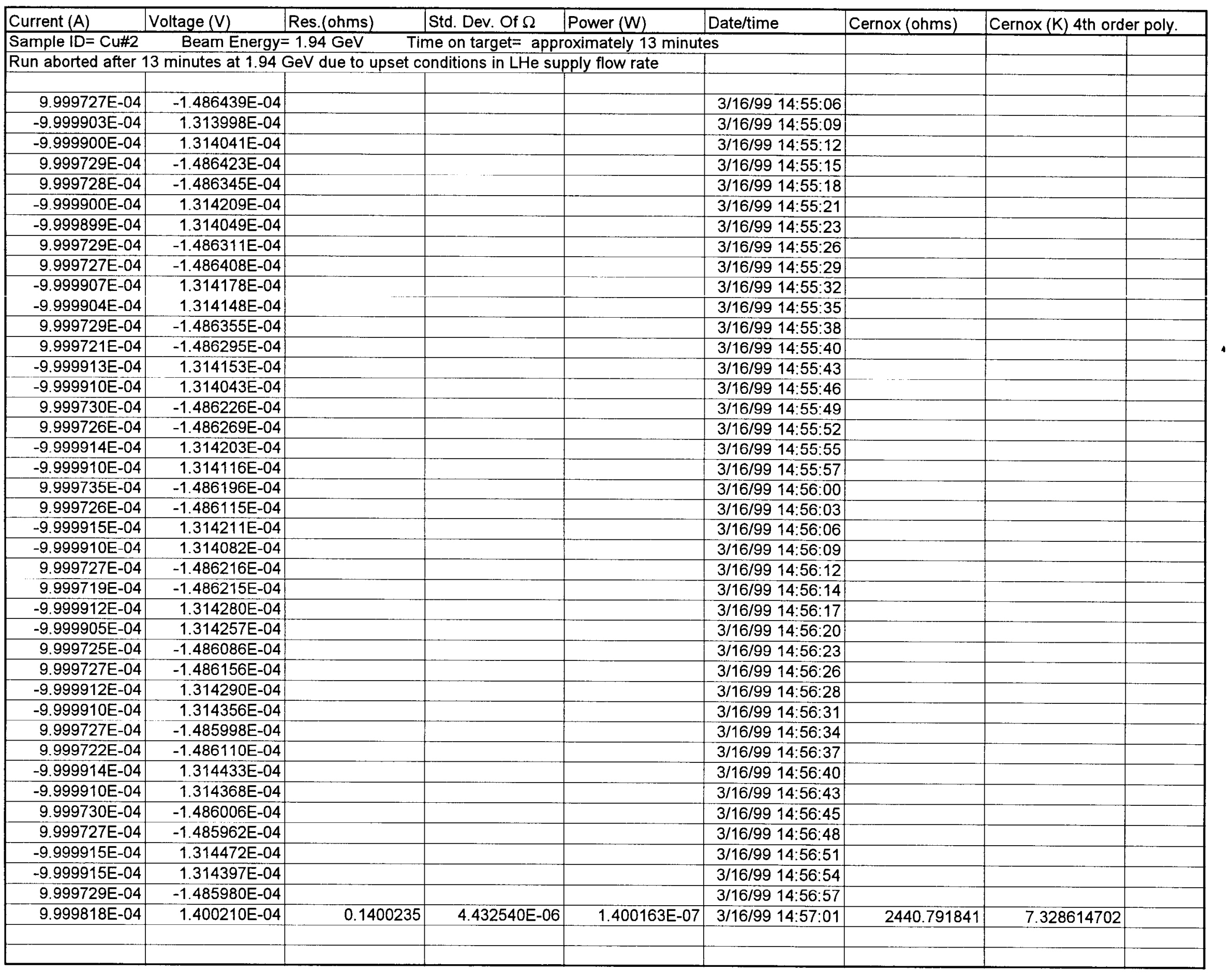




\begin{tabular}{|c|c|c|c|c|c|c|c|c|}
\hline 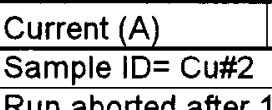 & Voltage (V) & Res.(ohms) & Std. Dev. Of $\Omega$ & $\frac{\mid \text { Power }(W)}{\text { roximately } 13 \text { minute }}$ & Date/time & Cernox (ohms) & \multicolumn{2}{|c|}{ Cernox (K) 4th order poly. } \\
\hline & & & & & & & & \\
\hline $9.999817 \mathrm{E}-03$ & $-1.408456 E-03$ & & & & $3 / 16 / 99$ 15:10:35 & & & \\
\hline$-9.999936 \mathrm{E}-03$ & $1.391329 \mathrm{E}-03$ & & & & $3 / 16 / 99$ 15:10:41 & & & \\
\hline $9.999814 \mathrm{E}-03$ & $-1.408442 E-03$ & & & & $3 / 16 / 99$ 15:10:44 & & & \\
\hline $9.999809 \mathrm{E}-03$ & $-1.408420 \mathrm{E}-03$ & & & & $3 / 16 / 99$ 15:10:46 & & & \\
\hline$-9.999936 \mathrm{E}-03$ & $1.391329 \mathrm{E}-03$ & & & & $3 / 16 / 99$ 15:10:49 & & & \\
\hline $9.999816 \mathrm{E}-03$ & $-1.408400 \mathrm{E}-03$ & & & & $3 / 16 / 99 \quad 15: 10: 58$ & & & \\
\hline$-9.999933 E-03$ & $1.391346 \mathrm{E}-03$ & & & & $3 / 16 / 9915: 11: 01$ & & & \\
\hline$-9.999931 E-03$ & $1.391349 \mathrm{E}-03$ & & & & 3/16/99 15:11:03 & & & \\
\hline $9.999821 \mathrm{E}-03$ & $-1.408380 \mathrm{E}-03$ & & & & 3/16/99 15:11:06 & & & \\
\hline $9.999821 \mathrm{E}-03$ & $-1.408395 \mathrm{E}-03$ & & & & $3 / 16 / 99$ 15:11:09 & & & \\
\hline$-9.999928 \mathrm{E}-03$ & $1.391352 \mathrm{E}-03$ & & & & $3 / 16 / 99$ 15:11:12 & & & \\
\hline$-9.999925 E-03$ & $1.391361 \mathrm{E}-03$ & & & & $3 / 16 / 99$ 15:11:15 & & & \\
\hline $9.999812 \mathrm{E}-03$ & $-1.408357 \mathrm{E}-03$ & & & & $3 / 16 / 99$ 15:11:32 & & & \\
\hline$-9.999940 \mathrm{E}-03$ & $1.391357 \mathrm{E}-03$ & & & & $3 / 16 / 9915: 11: 34$ & & & \\
\hline$-9.999936 \mathrm{E}-03$ & $1.391356 \mathrm{E}-03$ & & & & $\begin{array}{lll}3 / 16 / 99 & 15: 11: 37 \\
\end{array}$ & & & \\
\hline $9.999817 \mathrm{E}-03$ & $-1.408355 E-03$ & & & & $3 / 16 / 9915: 11: 40$ & & & \\
\hline $9.999812 \mathrm{E}-03$ & $-1.408355 \mathrm{E}-03$ & & & & $3 / 16 / 99$ 15:11:43 & & & \\
\hline$-9.999936 \mathrm{E}-03$ & $1.391370 \mathrm{E}-03$ & & & & $3 / 16 / 9915: 11: 46$ & & & \\
\hline$-9.999932 \mathrm{E}-03$ & $1.391350 \mathrm{E}-03$ & & & & $3 / 16 / 99$ 15:11:49 & & & \\
\hline $9.999822 \mathrm{E}-03$ & $-1.408371 \mathrm{E}-03$ & & & & $3 / 16 / 99$ 15:11:51 & & & \\
\hline $9.999815 \mathrm{E}-03$ & $-1.408346 \mathrm{E}-03$ & & & & $3 / 16 / 99$ 15:11:54 & & & \\
\hline$-9.999938 \mathrm{E}-03$ & $1.391350 \mathrm{E}-03$ & & & & $3 / 16 / 99$ 15:11:57 & & & \\
\hline$-9.999931 \mathrm{E}-03$ & $1.391353 \mathrm{E}-03$ & & & & $3 / 16 / 99$ 15:12:00 & & & \\
\hline $9.999825 \mathrm{E}-03$ & $-1.408345 \mathrm{E}-03$ & & & & $3 / 16 / 99$ 15:12:03 & & & \\
\hline $9.999819 \mathrm{E}-03$ & $-1.408343 \mathrm{E}-03$ & & & & $3 / 16 / 9915: 12: 06$ & & & \\
\hline$-9.999935 E-03$ & $1.391356 \mathrm{E}-03$ & & & & $3 / 16 / 99$ 15:12:08 & & & \\
\hline
\end{tabular}


Copper2.xls

\begin{tabular}{|c|c|c|c|c|c|c|c|c|}
\hline Current $(A)$ & Voltage $(V)$ & Res.(ohms) & Std. Dev. Of $\Omega$ & Power (W) & Date/time & Cernox (ohms) & Cernox $(K)$ 4th orde & er poly. \\
\hline Sample ID=Cu\#2 & Beam Energy= & $=0 \quad$ Time on ta & arget $=0$ & & & & & \\
\hline Room Temperature & $\theta$ measurement betv & ween 1.94 and 1.1 & $\mathrm{GeV}$ & & & & & \\
\hline & & & & & & & & \\
\hline $5.000243 \mathrm{E}-05$ & $-8.718563 \mathrm{E}-04$ & & & & $3 / 16 / 9916: 19: 00$ & & & \\
\hline$-5.000595 E-05$ & $8.317844 \mathrm{E}-04$ & & & & $3 / 16 / 99$ 16:19:03 & & & \\
\hline$-5.000598 E-05$ & $8.319706 \mathrm{E}-04$ & & & & $3 / 16 / 99$ 16:19:06 & & & \\
\hline $5.000245 \mathrm{E}-05$ & $-8.715097 \mathrm{E}-04$ & & & & $3 / 16 / 99$ 16:19:09 & & & \\
\hline $5.000243 \mathrm{E}-05$ & $-8.713997 E-04$ & & & & $3 / 16 / 9916: 19: 11$ & & & \\
\hline$-5.000604 E-05$ & $8.325639 \mathrm{E}-04$ & & & & 3/16/99 16:19:14 & & & \\
\hline$-5.000606 \mathrm{E}-05$ & $8.327345 \mathrm{E}-04$ & & & & $3 / 16 / 9916: 19: 17$ & & & \\
\hline $5.000247 E-05$ & $-8.710710 \mathrm{E}-04$ & & & & $3 / 16 / 99$ 16:19:20 & & & \\
\hline $5.000250 \mathrm{E}-05$ & -8.709600 E-04 & & & & $3 / 16 / 9916: 19: 23$ & & & \\
\hline $5.000602 E-05$ & $8.333074 \mathrm{E}-04$ & & & & $3 / 16 / 9916: 19: 26$ & & & \\
\hline$-5.000607 \mathrm{E}-05$ & $8.335066 \mathrm{E}-04$ & & & & $3 / 16 / 9916: 19: 28$ & & & \\
\hline $5.000249 E-05$ & $-8.706229 \mathrm{E}-04$ & & & & $3 / 16 / 9916: 19: 31$ & & & \\
\hline $5.000250 \mathrm{E}-05$ & $-8.705156 \mathrm{E}-04$ & & & & $3 / 16 / 9916: 19: 34$ & & & \\
\hline$-5.000606 \mathrm{E}-05$ & $8.340669 \mathrm{E}-04$ & & & & $3 / 16 / 9916: 19: 37$ & & & \\
\hline$-5.000606 \mathrm{E}-05$ & $8.342159 E-04$ & & & & $3 / 16 / 9916: 19: 40$ & & & \\
\hline $5.000252 E-05$ & $-8.703014 \mathrm{E}-04$ & & & & $3 / 16 / 99$ 16:19:43 & & & \\
\hline $5.000250 \mathrm{E}-05$ & $-8.702662 E-04$ & & & & $3 / 16 / 9916: 19: 45$ & & & \\
\hline$-5.000599 E-05$ & $8.346008 \mathrm{E}-04$ & & & & $3 / 16 / 9916: 19: 48$ & & & \\
\hline$-5.000601 \mathrm{E}-05$ & $8.347015 E-04$ & & & & $3 / 16 / 9916: 19: 51$ & & & \\
\hline $5.000249 \mathrm{E}-05$ & $-8.702466 \mathrm{E}-04$ & & & & $3 / 16 / 9916: 19: 54$ & & & \\
\hline $5.000425 \mathrm{E}-05$ & $8.521101 \mathrm{E}-04$ & 17.0407532 & 4.789497E-03 & $4.262732 \mathrm{E}-08$ & $3 / 16 / 9916: 19: 58$ & 60.54076116 & 290.6932602 & \\
\hline & & & & & & & & \\
\hline & & & & & & & & \\
\hline
\end{tabular}


Copper2.xls

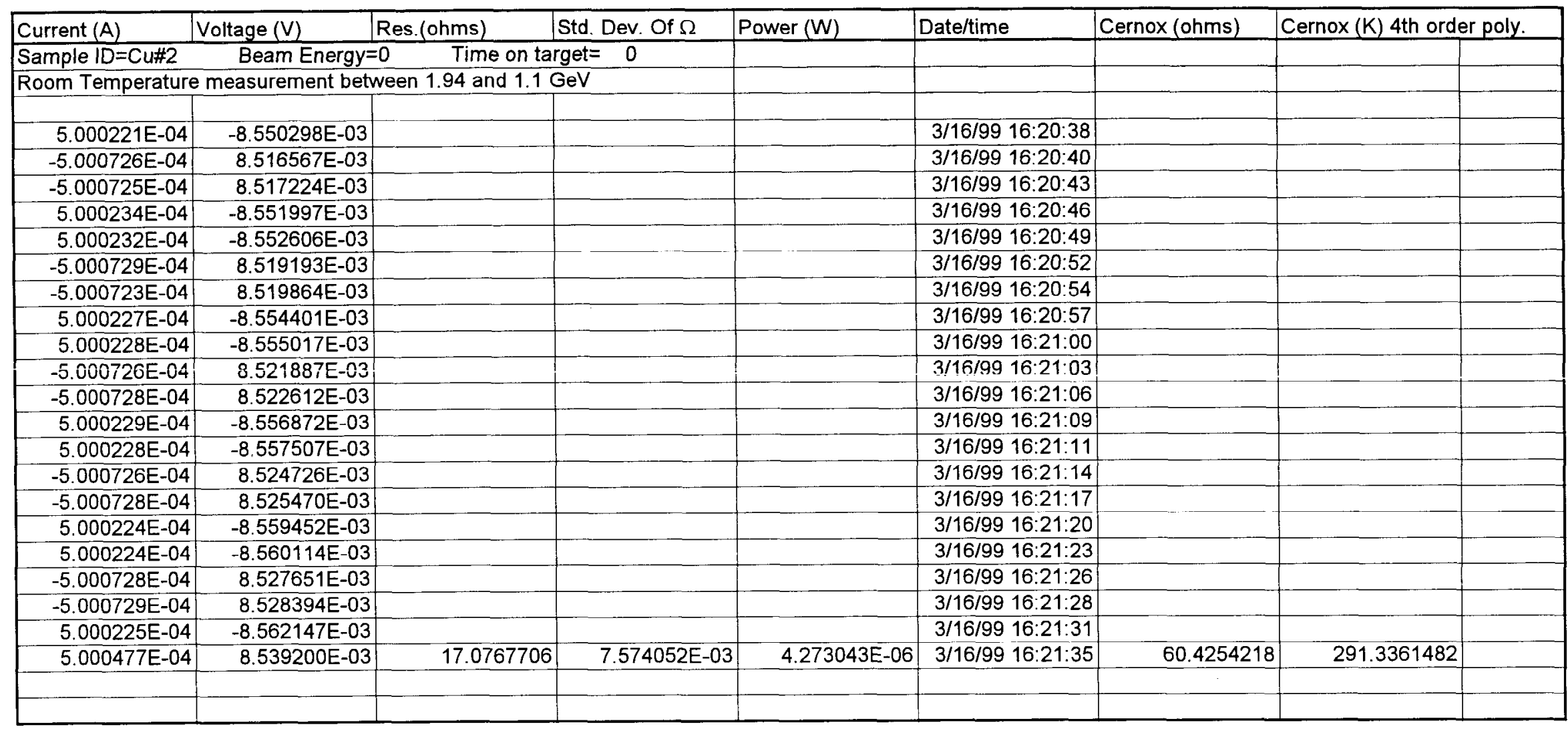


Copper2.xls

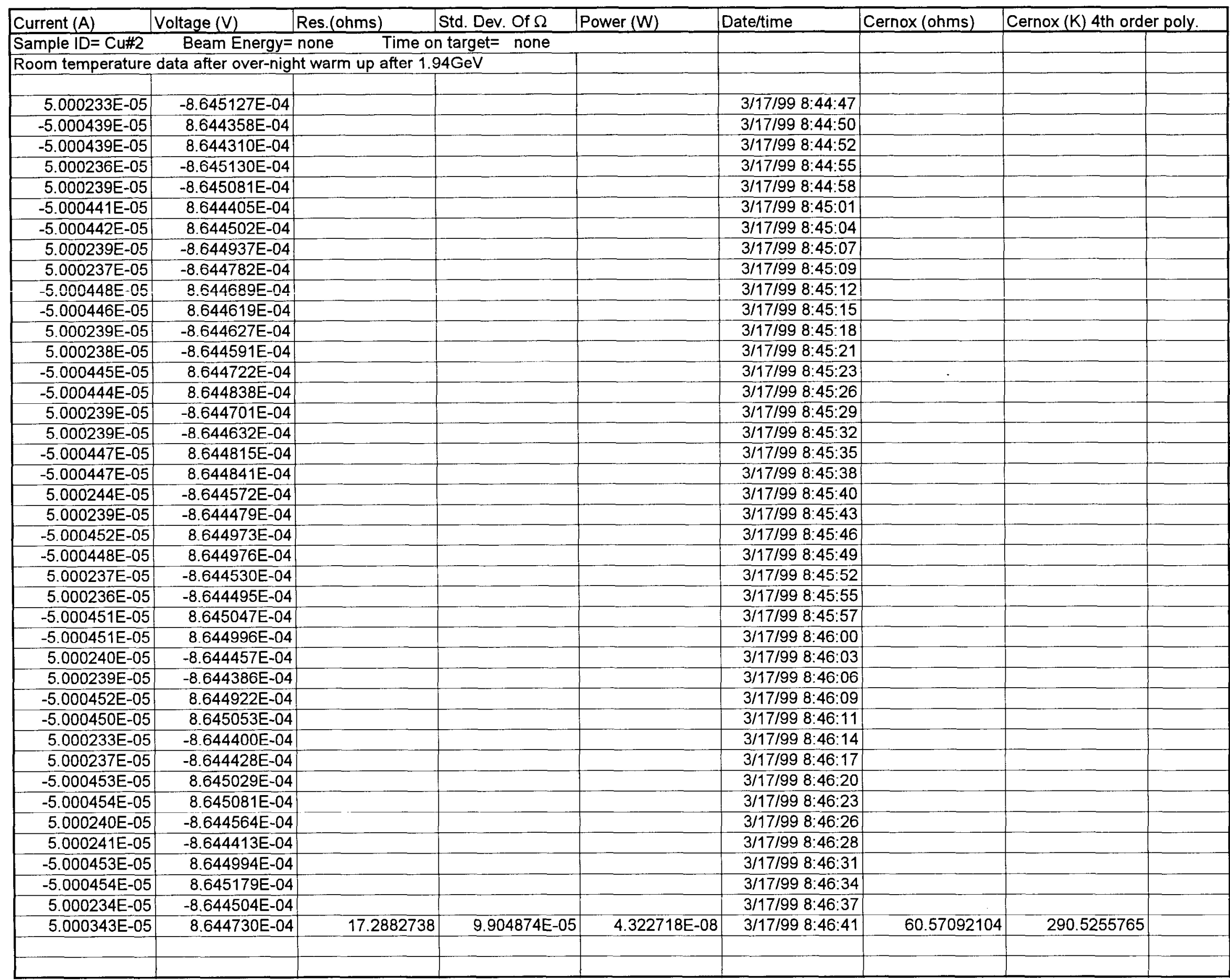


Copper2.xls

\begin{tabular}{|c|c|c|c|c|c|c|c|c|}
\hline Current (A) & Voltage (V) & Res.(ohms) & Std. Dev. Of $\Omega$ & Power (W) & Date/time & Cernox (ohms) & Cernox (K) 4th orde & er poly. \\
\hline Sample ID= Cu\#2 & Beam Energy= & Time on & $n$ target $=$ none & & & & & \\
\hline Room temperature & data after over-nigh & ht warm up after 1.9 & $94 \mathrm{GeV}$ & & & & & \\
\hline & & & & & & & & \\
\hline $5.000206 \mathrm{E}-04$ & $-8.644212 E-03$ & & & & $3 / 17 / 998: 47: 15$ & & & \\
\hline$-5.000577 \mathrm{E}-04$ & $8.644106 \mathrm{E}-03$ & & & & $3 / 17 / 998: 47: 18$ & & & \\
\hline$-5.000581 E-04$ & $8.644076 \mathrm{E}-03$ & & & & $3 / 17 / 998: 47: 21$ & & & \\
\hline $5.000212 \mathrm{E}-04$ & $-8.644250 \mathrm{E}-03$ & & & & $3 / 17 / 998: 47: 24$ & & & \\
\hline $5.000208 \mathrm{E}-04$ & $-8.644237 \mathrm{E}-03$ & & & & $3 / 17 / 998: 47: 26$ & & & \\
\hline$-5.000582 E-04$ & $8.644087 \mathrm{E}-03$ & & & & $3 / 17 / 998: 47: 29$ & & & \\
\hline$-5.000577 \mathrm{E}-04$ & 8.644090E-03 & & & & $3 / 17 / 998: 47: 32$ & & & \\
\hline $5.000209 \mathrm{E}-04$ & $-8.644273 E-03$ & & & & $3 / 17 / 998: 47: 35$ & & & \\
\hline $5.000212 \mathrm{E}-04$ & $-8.644264 \mathrm{E}-03$ & & & & $3 / 17 / 998: 47: 38$ & & & \\
\hline-5.000577 E-04 & $8.644105 \mathrm{E}-03$ & & & & $3 / 17 / 998: 47: 41$ & & & \\
\hline$-5.000576 \mathrm{E}-04$ & $8.644111 \mathrm{E}-03$ & & & & $3 / 17 / 998: 47: 43$ & & & \\
\hline $5.000210 \mathrm{E}-04$ & $-8.644243 E-03$ & & & & $3 / 17 / 998: 47: 46$ & & & \\
\hline $5.000211 \mathrm{E}-04$ & $-8.644243 E-03$ & & & & $3 / 17 / 998: 47: 49$ & & & \\
\hline$-5.000577 \mathrm{E}-04$ & $8.644104 \mathrm{E}-03$ & & & & $3 / 17 / 998: 47: 52$ & & & \\
\hline$-5.000578 E-04$ & $8.644141 E-03$ & & & & $3 / 17 / 998: 47: 55$ & & & \\
\hline $5.000210 \mathrm{E}-04$ & $-8.644223 E-03$ & & & & $3 / 17 / 998: 47: 57$ & & & \\
\hline $5.000207 \mathrm{E}-04$ & $-8.644234 \mathrm{E}-03$ & & & & $3 / 17 / 998: 48: 00$ & & & \\
\hline$-5.000582 E-04$ & $8.644147 E-03$ & & & & $3 / 17 / 998: 48: 03$ & & & \\
\hline$-5.000585 E-04$ & $8.644162 \mathrm{E}-03$ & & & & $3 / 17 / 998: 48: 06$ & & & \\
\hline $5.000209 \mathrm{E}-04$ & $-8.644236 \mathrm{E}-03$ & & & & $3 / 17 / 998: 48: 09$ & & & \\
\hline $5.000207 \mathrm{E}-04$ & $-8.644235 E-03$ & & & & $3 / 17 / 998: 48: 12$ & & & \\
\hline$-5.000585 E-04$ & $8.644167 \mathrm{E}-03$ & & & & $3 / 17 / 998: 48: 14$ & & & \\
\hline$-5.000585 \mathrm{E}-04$ & $8.644180 \mathrm{E}-03$ & & & & $3 / 17 / 998: 48: 17$ & & & \\
\hline $5.000208 \mathrm{E}-04$ & $-8.644232 E-03$ & & & & $3 / 17 / 998: 48: 20$ & & & \\
\hline $5.000207 \mathrm{E}-04$ & $-8.644242 \mathrm{E}-03$ & & & & $3 / 17 / 998: 48: 23$ & & & \\
\hline$-5.000587 \mathrm{E}-04$ & $8.644185 \mathrm{E}-03$ & & & & $3 / 17 / 998: 48: 26$ & & & \\
\hline$-5.000587 E-04$ & $8.644188 \mathrm{E}-03$ & & & & 3/17/99 8:48:29 & & & \\
\hline $5.000203 \mathrm{E}-04$ & $-8.644207 \mathrm{E}-03$ & & & & $3 / 17 / 998: 48: 31$ & & & \\
\hline $5.000205 E-04$ & $-8.644223 E-03$ & & & & $3 / 17 / 998: 48: 34$ & & & \\
\hline$-5.000586 \mathrm{E}-04$ & $8.644195 \mathrm{E}-03$ & & & & $3 / 17 / 998: 48: 37$ & & & \\
\hline$-5.000588 \mathrm{E}-04$ & $8.644195 \mathrm{E}-03$ & & & & $3 / 17 / 998: 48: 40$ & & & \\
\hline $5.000206 \mathrm{E}-04$ & $-8.644265 E-03$ & & & & $3 / 17 / 998: 48: 43$ & & & \\
\hline $5.000207 \mathrm{E}-04$ & $-8.644246 \mathrm{E}-03$ & & & & $3 / 17 / 998: 48: 46$ & & & \\
\hline$-5.000588 \mathrm{E}-04$ & $8.644178 \mathrm{E}-03$ & & & & $3 / 17 / 998: 48: 48$ & & & \\
\hline$-5.000586 \mathrm{E}-04$ & $8.644188 \mathrm{E}-03$ & & & & $3 / 17 / 998: 48: 51$ & & & \\
\hline $5.000203 E-04$ & $-8.644259 \mathrm{E}-03$ & & & & $3 / 17 / 998: 48: 54$ & & & \\
\hline $5.000205 E-04$ & $-8.644250 \mathrm{E}-03$ & & & & $3 / 17 / 998: 48: 57$ & & & \\
\hline$-5.000590 \mathrm{E}-04$ & $8.644201 E-03$ & & & & $3 / 17 / 998: 49: 00$ & & & \\
\hline$-5.000590 \mathrm{E}-04$ & $8.644212 E-03$ & & & & $3 / 17 / 998: 49: 02$ & & & \\
\hline $5.000202 E-04$ & $-8.644224 \mathrm{E}-03$ & & & & $3 / 17 / 998: 49: 05$ & & & \\
\hline $5.000395 \mathrm{E}-04$ & $8.644195 \mathrm{E}-03$ & 17.2870241 & $3.935861 \mathrm{E}-05$ & 4.322451E-06 & 3/17/99 8:49:09 & 60.58316055 & 290.4575769 & \\
\hline & & & & & & & & \\
\hline
\end{tabular}




\begin{tabular}{|c|c|c|c|c|c|c|c|c|}
\hline Current $(A)$ & Voltage (V) & Res.(ohms) & Std. Dev. Of $\Omega$ & Power (W) & Date/time & Cernox (ohms) & Cernox $(K)$ 4th ord & er poly. \\
\hline Sample ID = Cu\#2 & Beam Energy= & $=$ pre-1.1GeV & Time on target $=0$ & & & & & \\
\hline This is the cold dat & ta acquisition at 4.72 & $2 \mathrm{~K}$ prior to irradiatio & on at $1.1 \mathrm{GeV}$ & & & & & \\
\hline & & & & & & & & \\
\hline $5.000167 \mathrm{E}-03$ & $-7.000423 E-04$ & & & & $3 / 17 / 99$ 10:08:59 & & & \\
\hline$-5.000692 \mathrm{E}-03$ & $6.855439 \mathrm{E}-04$ & & & & $3 / 17 / 99$ 10:09:02 & & & \\
\hline$-5.000695 \mathrm{E}-03$ & $6.855723 \mathrm{E}-04$ & & & & 3/17/99 10:09:04 & & & \\
\hline $5.000170 \mathrm{E}-03$ & $-7.000474 \mathrm{E}-04$ & & & & 3/17/99 10:09:07 & & & \\
\hline $5.000172 \mathrm{E}-03$ & $-7.000535 E-04$ & & & & $3 / 17 / 9910: 09: 10$ & & & \\
\hline$-5.000691 E-03$ & $6.855428 \mathrm{E}-04$ & & & & $3 / 17 / 99$ 10:09:13 & & & \\
\hline$-5.000690 \mathrm{E}-03$ & $6.855622 E-04$ & & & & 3/17/99 10:09:16 & & & \\
\hline $5.000174 \mathrm{E}-03$ & $-7.000426 \mathrm{E}-04$ & & & & $3 / 17 / 99$ 10:09:19 & & & \\
\hline $5.000178 \mathrm{E}-03$ & $-7.000206 E-04$ & & & & $3 / 17 / 99 \quad 10: 09: 21$ & & & \\
\hline$-5.000690 \mathrm{E}-03$ & $6.855542 \mathrm{E}-04$ & & & & $3 / 17 / 9910: 09: 24$ & & & \\
\hline$-5.000697 \mathrm{E}-03$ & $6.855536 \mathrm{E}-04$ & & & & $3 / 17 / 99 \quad 10: 09: 27$ & & & \\
\hline $5.000179 \mathrm{E}-03$ & $-7.000294 E-04$ & & & & $3 / 17 / 99$ 10:09:30 & & & \\
\hline $5.000183 E-03$ & $-7.000218 E-04$ & & & & $3 / 17 / 9910: 09: 33$ & & & \\
\hline$-5.000696 \mathrm{E}-03$ & $6.855576 \mathrm{E}-04$ & & & & $3 / 17 / 9910: 09: 35$ & & & \\
\hline$-5.000691 \mathrm{E}-03$ & $6.855697 \mathrm{E}-04$ & & & & $3 / 17 / 9910: 09: 38$ & & & \\
\hline $5.000182 \mathrm{E}-03$ & $-7.000291 E-04$ & & & & $3 / 17 / 9910: 09: 41$ & & & \\
\hline 5.000175E-03 & $-7.000301 E-04$ & & & & $3 / 17 / 9910: 09: 44$ & & & \\
\hline$-5.000682 \mathrm{E}-03$ & $6.855762 \mathrm{E}-04$ & & & & $3 / 17 / 9910: 09: 47$ & & & \\
\hline$-5.000685 E-03$ & $6.855654 \mathrm{E}-04$ & & & & $3 / 17 / 9910: 09: 50$ & & & \\
\hline $5.000182 \mathrm{E}-03$ & $-7.000181 \mathrm{E}-04$ & & & & $3 / 17 / 99$ 10:09:52 & & & \\
\hline $5.000180 \mathrm{E}-03$ & $-7.000219 E-04$ & & & & $3 / 17 / 9910: 09: 55$ & & & \\
\hline$-5.000681 E-03$ & $6.855728 \mathrm{E}-04$ & & & & $3 / 17 / 9910: 09: 58$ & & & \\
\hline$-5.000685 E-03$ & $6.855665 \mathrm{E}-04$ & & & & $3 / 17 / 99$ 10:10:01 & & & \\
\hline $5.000180 \mathrm{E}-03$ & $-7.000195 E-04$ & & & & $3 / 17 / 9910: 10: 04$ & & & \\
\hline $5.000182 \mathrm{E}-03$ & $-7.000230 E-04$ & & & & $3 / 17 / 9910: 10: 07$ & & & \\
\hline$-5.000686 \mathrm{E}-03$ & $6.855755 \mathrm{E}-04$ & & & & $3 / 17 / 99$ 10:10:09 & & & \\
\hline$-5.000686 \mathrm{E}-03$ & $6.855706 \mathrm{E}-04$ & & & & $3 / 17 / 99$ 10:10:12 & & & \\
\hline $5.000184 \mathrm{E}-03$ & $-7.000166 \mathrm{E}-04$ & & & & $3 / 17 / 9910: 10: 15$ & & & \\
\hline $5.000183 \mathrm{E}-03$ & $-7.000310 \mathrm{E}-04$ & & & & $3 / 17 / 99$ 10:10:18 & & & \\
\hline$-5.000683 \mathrm{E}-03$ & $6.855860 \mathrm{E}-04$ & & & & $3 / 17 / 99$ 10:10:21 & & & \\
\hline$-5.000681 E-03$ & $6.855817 \mathrm{E}-04$ & & & & $3 / 17 / 9910: 10: 24$ & & & \\
\hline 5.000183E-03 & $-7.000079 \mathrm{E}-04$ & & & & $3 / 17 / 99$ 10:10:26 & & & \\
\hline $5.000178 \mathrm{E}-03$ & $-7.000103 E-04$ & & & & 3/17/99 10:10:29 & & & \\
\hline$-5.000692 E-03$ & $6.855848 E-04$ & & & & $3 / 17 / 99 \quad 10: 10: 32$ & & & \\
\hline$-5.000695 \mathrm{E}-03$ & $6.855751 \mathrm{E}-04$ & & & & 3/17/99 10:10:35 & & & \\
\hline $5.000180 \mathrm{E}-03$ & $-7.000129 \mathrm{E}-04$ & & & & $3 / 17 / 99$ 10:10:38 & & & \\
\hline $5.000178 \mathrm{E}-03$ & $-7.000266 \mathrm{E}-04$ & & & & $3 / 17 / 9910: 10: 40$ & & & \\
\hline$-5.000693 E-03$ & $6.855720 \mathrm{E}-04$ & & & & $3 / 17 / 99$ 10:10:43 & & & \\
\hline$-5.000691 E-03$ & $6.855891 \mathrm{E}-04$ & & & & $3 / 17 / 99$ 10:10:46 & & & \\
\hline $5.000180 \mathrm{E}-03$ & $-7.000191 \mathrm{E}-04$ & & & & $3 / 17 / 99$ 10:10:49 & & & \\
\hline $5.000434 \mathrm{E}-03$ & $6.927974 \mathrm{E}-04$ & 0.1385475 & $1.199258 \mathrm{E}-06$ & 3.464322E-06 & $3 / 17 / 99$ 10:10:53 & 4974.969991 & 4.727545519 & \\
\hline & & & & & & & & \\
\hline
\end{tabular}


Copper2.xls

\begin{tabular}{|c|c|c|c|c|c|c|c|c|}
\hline Current (A) & Voltage (V) & Res.(ohms) & Std. Dev. Of $\Omega$ & Power (W) & Date/time & Cernox (ohms) & \multicolumn{2}{|c|}{ Cernox (K) 4th order poly. } \\
\hline Sample ID= Cu\#2 & Beam Energy $=$ & $=$ pre $-1.1 \mathrm{GeV}$ & Time on target $=0$ & & & & & \\
\hline \multicolumn{9}{|c|}{ This is the cold data acquisition at $4.72 \mathrm{~K}$ prior to irradiation at $1.1 \mathrm{GeV}$} \\
\hline & & & & & & & & \\
\hline $9.999768 \mathrm{E}-03$ & $-1.392687 E-03$ & & & & $3 / 17 / 99$ 10:11:28 & & & \\
\hline$-1.000000 \mathrm{E}-02$ & $1.378201 \mathrm{E}-03$ & & & & $3 / 17 / 9910: 11: 31$ & & & \\
\hline$-9.999995 E-03$ & $1.378194 \mathrm{E}-03$ & & & & $3 / 17 / 99$ 10:11:34 & & & \\
\hline 9.999773E-03 & $-1.392700 \mathrm{E}-03$ & & & & $3 / 17 / 99$ 10:11:37 & & & \\
\hline $9.999773 \mathrm{E}-03$ & $-1.392711 \mathrm{E}-03$ & & & & $3 / 17 / 9910: 11: 40$ & & & \\
\hline$-1.000000 \mathrm{E}-02$ & $1.378207 \mathrm{E}-03$ & & & & $3 / 17 / 99$ 10:11:42 & & & \\
\hline$-9.999996 \mathrm{E}-03$ & $1.378211 \mathrm{E}-03$ & & & & $3 / 17 / 9910: 11: 45$ & & & \\
\hline $9.999778 \mathrm{E}-03$ & $-1.392707 \mathrm{E}-03$ & & & & $3 / 17 / 99$ 10:11:48 & & & \\
\hline $9.999771 \mathrm{E}-03$ & $-1.392704 \mathrm{E}-03$ & & & & $3 / 17 / 9910: 11: 51$ & & & \\
\hline$-1.000000 \mathrm{E}-02$ & $1.378188 \mathrm{~F}-03$ & & & & $3 / 17 / 99$ 10:11:54 & & & \\
\hline$-9.999997 E-03$ & $1.378215 \mathrm{E}-03$ & & & & $3 / 17 / 9910: 11: 57$ & & & \\
\hline 9.999773E-03 & $-1.392713 \mathrm{E}-03$ & & & & $3 / 17 / 99$ 10:11:59 & & & \\
\hline $9.999770 \mathrm{E}-03$ & $-1.392718 E-03$ & & & & $3 / 17 / 99$ 10:12:02 & & & \\
\hline$-1.000000 \mathrm{E}-02$ & $1.378206 \mathrm{E}-03$ & & & & $3 / 17 / 99 \quad 10: 12: 05$ & & & \\
\hline$-1.000000 E-02$ & $1.378201 \mathrm{E}-03$ & & & & $3 / 17 / 99 \quad 10: 12: 08$ & & & \\
\hline $9.999777 \mathrm{E}-03$ & $-1.392700 \mathrm{E}-03$ & & & & 3/17/99 10:12:11 & & & \\
\hline $9.999774 \mathrm{E}-03$ & $-1.392714 \mathrm{E}-03$ & & & & $3 / 17 / 9910: 12: 14$ & & & \\
\hline$-1.000000 \mathrm{E}-02$ & $1.378208 \mathrm{E}-03$ & & & & $3 / 17 / 99$ 10:12:16 & & & \\
\hline$-1.000000 E-02$ & 1.378189E-03 & & & & $3 / 17 / 9910: 12: 19$ & & & \\
\hline 9.999771E-03 & $-1.392713 \mathrm{E}-03$ & & & & $3 / 17 / 99 \quad 10: 12: 22$ & & & \\
\hline $9.999765 \mathrm{E}-03$ & $-1.392716 \mathrm{E}-03$ & & & & $3 / 17 / 9910: 12: 25$ & & & \\
\hline$-1.000001 E-02$ & $1.378196 \mathrm{E}-03$ & & & & $3 / 17 / 9910: 12: 28$ & & & \\
\hline$-1.000000 \mathrm{E}-02$ & $1.378206 \mathrm{E}-03$ & & & & $3 / 17 / 9910: 12: 30$ & & & \\
\hline $9.999775 \mathrm{E}-03$ & $-1.392705 \mathrm{E}-03$ & & & & $3 / 17 / 9910: 12: 33$ & & & \\
\hline $9.999768 \mathrm{E}-03$ & $-1.392710 \mathrm{E}-03$ & & & & $3 / 17 / 99$ 10:12:36 & & & \\
\hline$-1.000000 \mathrm{E}-02$ & 1.378213E-03 & & & & $3 / 17 / 9910: 12: 39$ & & & \\
\hline$-9.999998 E-03$ & $1.378188 \mathrm{E}-03$ & & & & $3 / 17 / 99 \quad 10: 12: 42$ & & & \\
\hline $9.999783 \mathrm{E}-03$ & $-1.392725 \mathrm{E}-03$ & & & & $3 / 47 / 9910: 12: 45$ & & & \\
\hline $9.999774 \mathrm{E}-03$ & $-1.392722 \mathrm{E}-03$ & & & & $3 / 17 / 99 \quad 10: 12: 47$ & & & \\
\hline$-9.999999 E-03$ & $1.378183 \mathrm{E}-03$ & & & & $3 / 17 / 99 \quad 10: 12: 50$ & & & \\
\hline$-9.999990 \mathrm{E}-03$ & $1.378195 \mathrm{E}-03$ & & & & $3 / 17 / 99$ 10:12:53 & & & \\
\hline $9.999781 \mathrm{E}-03$ & $-1.392726 E-03$ & & & & $3 / 17 / 99 \quad 10: 12: 56$ & & & \\
\hline $9.999778 \mathrm{E}-03$ & $-1.392708 \mathrm{E}-03$ & & & & $3 / 17 / 99$ 10:12:59 & & & \\
\hline$-1.000000 \mathrm{E}-02$ & 1.378192E-03 & & & & $3 / 17 / 9910: 13: 02$ & & & \\
\hline$-9.999998 E-03$ & $1.378193 \mathrm{E}-03$ & & & & $3 / 17 / 9910: 13: 04$ & & & \\
\hline 9.999782E-03 & $-1.392725 E-03$ & & & & $3 / 17 / 99$ 10:13:07 & & & \\
\hline $9.999779 \mathrm{E}-03$ & $-1.392721 \mathrm{E}-03$ & & & & $3 / 17 / 99$ 10:13:10 & & & \\
\hline$-1.000000 \mathrm{E}-02$ & $1.378191 \mathrm{E}-03$ & & & & $3 / 17 / 99$ 10:13:13 & & & \\
\hline$-1.000000 \mathrm{E}-02$ & $1.378197 \mathrm{E}-03$ & & & & $3 / 17 / 9910: 13: 16$ & & & \\
\hline $9.999784 E-03$ & $-1.392732 \mathrm{E}-03$ & & & & $3 / 17 / 99$ 10:13:19 & & & \\
\hline $9.999887 \mathrm{E}-03$ & $1.385456 \mathrm{E}-03$ & 0.1385471 & $5.792271 \mathrm{E}-07$ & 1.385449E-05 & $3 / 17 / 99$ 10:13:23 & 4974.413082 & 4.727842235 & \\
\hline & & & & & & & & \\
\hline
\end{tabular}


Copper2.xis

\begin{tabular}{|c|c|c|c|c|c|c|c|c|}
\hline Current (A) & Voltage (V) & Res.(ohms) & Std. Dev. Of $\Omega$ & Power (W) & Date/time & Cernox (ohms) & Cernox (K) 4th orde & er poly. \\
\hline Sample ID= Cu\#2 & Beam Energy= & $=$ pre- $1.1 \mathrm{GeV}$ & Time on target $=0$ & & & & & \\
\hline \multicolumn{9}{|c|}{ This is the cold data acquisition at $4.72 \mathrm{~K}$ prior to irradiation at $1.1 \mathrm{GeV}$} \\
\hline & & & & & & & & \\
\hline $1.499861 \mathrm{E}-02$ & $-2.085521 E-03$ & & & & $3 / 17 / 99$ 10:13:57 & & & \\
\hline$-1.500289 E-02$ & $2.071090 \mathrm{E}-03$ & & & & $3 / 17 / 9910: 14: 00$ & & & \\
\hline$-1.500285 E-02$ & $2.071097 \mathrm{E}-03$ & & & & $3 / 17 / 9910: 14: 03$ & & & \\
\hline $1.499868 \mathrm{E}-02$ & $-2.085514 \mathrm{E}-03$ & & & & $3 / 17 / 99 \quad 10: 14: 05$ & & & \\
\hline $1.499867 \mathrm{E}-02$ & $-2.085511 \mathrm{E}-03$ & & - & & $3 / 17 / 9910: 14: 08$ & & & \\
\hline$-1.500283 E-02$ & $2.071090 \mathrm{E}-03$ & & - & & $3 / 17 / 9910: 14: 11$ & & & \\
\hline$-1.500289 \mathrm{E}-02$ & $2.071090 \mathrm{E}-03$ & & & & $3 / 17 / 9910: 14: 14$ & & & \\
\hline $1.499863 E-02$ & $-2.085507 E-03$ & & & & $3 / 17 / 9910: 14: 17$ & & & \\
\hline $1.499868 \mathrm{E}-02$ & $-2.085534 \mathrm{E}-03$ & & & & $3 / 17 / 9910: 14: 20$ & & & \\
\hline$-1.500261 E-02$ & $2.071073 E-03$ & & & \multirow{2}{*}{-} & $3 / 17 / 9910: 14: 22$ & & & \\
\hline$-1.500283 E-02$ & $2.071095 \mathrm{E}-03$ & & & & $3 / 17 / 9910: 14: 25$ & & & \\
\hline $1.499870 \mathrm{E}-02$ & $-2.085525 E-03$ & & & & $3 / 17 / 9910: 14: 28$ & & & \\
\hline 1.499872E-02 & $-2.085567 \mathrm{E}-03$ & & & & $3 / 17 / 99$ 10:14:31 & & & \\
\hline$-1.500289 E-02$ & $2.071066 \mathrm{E}-03$ & & & & $3 / 17 / 9910: 14: 34$ & & & \\
\hline$-1.500289 \mathrm{E}-02$ & $2.071076 \mathrm{E}-03$ & & & & $3 / 17 / 9910: 14: 37$ & & & \\
\hline $1.499870 \mathrm{E}-02$ & $-2.085537 E-03$ & & & & $\overline{3} / 17 / 9910: 14: 39$ & & & \\
\hline $1.499867 \mathrm{E}-02$ & $-2.085542 E-03$ & & & & $3 / 17 / 9910: 14: 42$ & & & \\
\hline$-1.500283 E-02$ & $2.071076 \mathrm{E}-03$ & & & & $3 / 17 / 99 \quad 10: 14: 45$ & & & \\
\hline$-1.500283 \mathrm{E}-02$ & $2.071062 \mathrm{E}-03$ & & & & $3 / 17 / 9910: 14: 48$ & & & \\
\hline 1.499870E-02 & $-2.085541 \mathrm{E}-03$ & & & & $3 / 17 / 9910: 14: 51$ & & & \\
\hline $1.499877 \mathrm{E}-02$ & $-2.085542 E-03$ & & & & $3 / 17 / 9910: 14: 54$ & & & \\
\hline$-1.500281 E-02$ & $2.071058 \mathrm{E}-03$ & & & & $3 / 17 / 9910: 14: 56$ & & & \\
\hline$-1.500282 E-02$ & $2.071072 \mathrm{E}-03$ & & & & $3 / 17 / 9910: 14: 59$ & & & \\
\hline $1.499877 \mathrm{E}-02$ & $-2.085558 \mathrm{E}-03$ & & & & $3 / 17 / 99 \quad 10: 15: 02$ & & & \\
\hline $1.499876 \mathrm{E}-02$ & $-2.085535 E-03$ & & & & $3 / 17 / 99 \quad 10: 15: 05$ & & & \\
\hline$-1.500276 \mathrm{E}-02$ & $2.071068 \mathrm{E}-03$ & & & & $3 / 17 / 9910: 15: 08$ & & & \\
\hline$-1.500277 \mathrm{E}-02$ & $2.071062 \mathrm{E}-03$ & & & & $3 / 17 / 9910: 15: 10$ & & & \\
\hline $1.499877 \mathrm{E}-02$ & $-2.085551 \mathrm{E}-03$ & & & & $3 / 17 / 9910: 15: 13$ & & & \\
\hline $1.499874 \mathrm{E}-02$ & $-2.085529 \mathrm{E}-03$ & & & & $3 / 17 / 9910: 15: 16$ & & & \\
\hline$-1.500284 E-02$ & $2.071088 \mathrm{E}-03$ & & & & $3 / 17 / 9910: 15: 19$ & & & \\
\hline$-1.500285 E-02$ & $2.071084 \mathrm{E}-03$ & & & & $3 / 17 / 9910: 15: 22$ & & & \\
\hline $1.499873 \mathrm{E}-02$ & $-2.085539 E-03$ & & & & $3 / 17 / 9910: 15: 25$ & & & \\
\hline $1.499875 \mathrm{E}-02$ & $-2.085538 \mathrm{E}-03$ & & & & $3 / 17 / 9910: 15: 27$ & & & \\
\hline$-1.500282 \mathrm{E}-02$ & $2.071060 \mathrm{E}-03$ & & & & $3 / 17 / 9910: 15: 30$ & & & \\
\hline$-1.500280 E-02$ & $2.071061 \mathrm{E}-03$ & & & & $3 / 17 / 9910: 15: 33$ & \multirow{2}{*}{$\ldots$} & & \\
\hline $1.499864 \mathrm{E}-02$ & $-2.085539 \mathrm{E}-03$ & & & & $3 / 17 / 9910: 15: 36$ & & & \\
\hline $1.499869 \mathrm{E}-02$ & $-2.085514 \mathrm{E}-03$ & & & & $3 / 17 / 99 \quad 10: 15: 39$ & & & \\
\hline$-1.500288 E-02$ & $2.071068 \mathrm{E}-03$ & & & & $3 / 17 / 9910: 15: 42$ & & & \\
\hline$-1.500283 \mathrm{E}-02$ & $2.071096 \mathrm{E}-03$ & & & & $3 / 17 / 9910: 15: 44$ & & & \\
\hline $1.499871 \mathrm{E}-02$ & $-2.085505 E-03$ & & & & $3 / 17 / 9910: 15: 47$ & & & \\
\hline $1.500077 \mathrm{E}-02$ & $2.078305 E-03$ & 0.1385465 & $3.844021 \mathrm{E}-07$ & $3.117612 \mathrm{E}-05$ & $3 / 17 / 9910: 15: 51$ & 4975.396127 & 4.727318518 & \\
\hline & & & & & & & & \\
\hline & & & & & & & & \\
\hline
\end{tabular}




\begin{tabular}{|c|c|c|c|c|c|c|c|c|}
\hline Current (A) & Voltage $(\mathrm{V})$ & Res.(ohms) & Std. Dev. Of $\Omega$ & Power (W) & Date/time & Cernox (ohms) & \multicolumn{2}{|c|}{ Cernox (K) 4th order poly. } \\
\hline Sample ID = Cu\#2 & \multicolumn{2}{|c|}{ Beam Energy= $1.1 \mathrm{GeV}$} & \multicolumn{2}{|c|}{ Time on target $=60$ minutes } & & & & \\
\hline \multicolumn{9}{|c|}{ Data at the end of the first irradiation at $1.1 \mathrm{GeV}$} \\
\hline & & & & & & & & \\
\hline $5.000179 \mathrm{E}-03$ & $-7.006942 \mathrm{E}-04$ & & & & $3 / 17 / 99$ 13:16:38 & & & \\
\hline$-5.000673 E-03$ & $6.859018 \mathrm{E}-04$ & & & & $3 / 17 / 9913: 16: 41$ & & & \\
\hline$-5.000667 \mathrm{E}-03$ & $6.858916 \mathrm{E}-04$ & & & & $3 / 17 / 9913: 16: 44$ & & & \\
\hline $5.000185 E-03$ & $-7.006988 E-04$ & & & & $3 / 17 / 99$ 13:16:47 & & & \\
\hline $5.000183 \mathrm{E}-03$ & $-7.007027 \mathrm{E}-04$ & & & & 3/17/99 13:16:50 & & & \\
\hline$-5.000666 \mathrm{E}-03$ & $6.858881 E-04$ & & & & $3 / 17 / 9913: 16: 52$ & & & \\
\hline$-5.000670 \mathrm{E}-03$ & $6.858854 \mathrm{E}-04$ & & & & $3 / 17 / 9913: 16: 55$ & & & \\
\hline $5.000187 \mathrm{E}-03$ & $-7.007054 \mathrm{E}-04$ & & & & 3/17/99 13:16:58 & & & \\
\hline $5.000187 \mathrm{E}-03$ & $-7.007150 \mathrm{E}-04$ & & & & $3 / 17 / 99$ 13:17:01 & & & \\
\hline$-5.000668 \mathrm{E}-03$ & $6.858961 \mathrm{E}-04$ & & & & 3/17/99 13:17:04 & & & \\
\hline$-5.000667 \mathrm{E}-03$ & $6.858838 \mathrm{E}-04$ & & & & 3/17/99 13:17:07 & & & \\
\hline $5.000188 \mathrm{E}-03$ & $-7.007182 E-04$ & & & & 3/17/99 13:17:09 & & & \\
\hline $5.000185 E-03$ & $-7.007131 \mathrm{E}-04$ & & & & $3 / 17 / 99$ 13:17:12 & & & \\
\hline$-5.000676 \mathrm{E}-03$ & $6.858759 \mathrm{E}-04$ & & & & $3 / 17 / 99$ 13:17:15 & & & \\
\hline$-5.000674 \mathrm{E}-03$ & $6.858640 \mathrm{E}-04$ & & & & $3 / 17 / 99$ 13:17:18 & & & \\
\hline $5.000187 \mathrm{E}-03$ & $-7.007281 E-04$ & & & & 3/17/99 13:17:21 & & & \\
\hline $5.000187 \mathrm{E}-03$ & $-7.007254 \mathrm{E}-04$ & & & & $3 / 17 / 99$ 13:17:23 & & & \\
\hline$-5.000670 \mathrm{E}-03$ & $6.858718 \mathrm{E}-04$ & & & & $3 / 17 / 9913: 17: 26$ & & & \\
\hline$-5.000678 \mathrm{E}-03$ & $6.858804 E-04$ & & & & $3 / 17 / 99$ 13:17:29 & & & \\
\hline $5.000186 \mathrm{E}-03$ & -7.007156 E-04 & & & & $3 / 17 / 99$ 13:17:32 & & & \\
\hline $5.000188 \mathrm{E}-03$ & $-7.007303 E-04$ & & & & $3 / 17 / 99$ 13:17:35 & & & \\
\hline$-5.000676 \mathrm{E}-03$ & $6.858655 \mathrm{E}-04$ & & & & $3 / 17 / 99$ 13:17:38 & & & \\
\hline$-5.000674 \mathrm{E}-03$ & $6.858647 \mathrm{E}-04$ & & & & $3 / 17 / 99$ 13:17:40 & & & \\
\hline $5.000191 \mathrm{E}-03$ & $-7.007329 E-04$ & & & & $3 / 17 / 9913: 17: 43$ & & & \\
\hline $5.000188 \mathrm{E}-03$ & $-7.007429 E-04$ & & & & $3 / 17 / 99$ 13:17:46 & & & \\
\hline$-5.000674 \mathrm{E}-03$ & $6.858539 \mathrm{E}-04$ & & & & $3 / 17 / 9913: 17: 49$ & & & \\
\hline$-5.000676 E-03$ & $6.858569 \mathrm{E}-04$ & & & & $3 / 17 / 9913: 17: 52$ & & . & \\
\hline $5.000183 E-03$ & $-7.007437 \mathrm{E}-04$ & & & & $3 / 17 / 99$ 13:17:55 & & & \\
\hline $5.000181 \mathrm{E}-03$ & $-7.007527 \mathrm{E}-04$ & & & & $3 / 17 / 99$ 13:17:57 & & & \\
\hline-5.000680 E-03 & $6.858445 \mathrm{E}-04$ & & & & 3/17/99 13:18:00 & & & \\
\hline$-5.000679 \mathrm{E}-03$ & $6.858423 E-04$ & & & & 3/17/99 13:18:03 & & & \\
\hline $5.000181 \mathrm{E}-03$ & $-7.007484 E-04$ & & & & $3 / 17 / 99$ 13:18:06 & & & \\
\hline $5.000181 \mathrm{E}-03$ & $-7.007535 \mathrm{E}-04$ & & & & $3 / 17 / 99$ 13:18:09 & & & \\
\hline$-5.000679 \mathrm{E}-03$ & $6.858384 \mathrm{E}-04$ & & & & $3 / 17 / 9913: 18: 12$ & & & \\
\hline$-5.000681 E-03$ & $6.858248 E-04$ & & & & $3 / 17 / 99$ 13:18:14 & & & \\
\hline $5.000178 \mathrm{E}-03$ & $-7.007594 \mathrm{E}-04$ & & & & $3 / 17 / 99$ 13:18:17 & & & \\
\hline $5.000182 \mathrm{E}-03$ & $-7.007584 \mathrm{E}-04$ & & & & $3 / 17 / 9913: 18: 20$ & & & \\
\hline$-5.000675 \mathrm{E}-03$ & $6.858246 \mathrm{E}-04$ & & & & $3 / 17 / 99$ 13:18:23 & & & \\
\hline$-5.000672 E-03$ & $6.858074 \mathrm{E}-04$ & & & & $3 / 17 / 9913: 18: 26$ & & & \\
\hline $5.000185 \mathrm{E}-03$ & $-7.007737 \mathrm{E}-04$ & & & & $3 / 17 / 9913: 18: 29$ & & & \\
\hline $5.000429 \mathrm{E}-03$ & $6.932969 E-04$ & 0.1386475 & $6.756555 \mathrm{E}-07$ & $3.466750 \mathrm{E}-06$ & 3/17/99 13:18:33 & 4990.809186 & 4.719131517 & \\
\hline & & & & & & & & \\
\hline & & & & & & & & \\
\hline
\end{tabular}




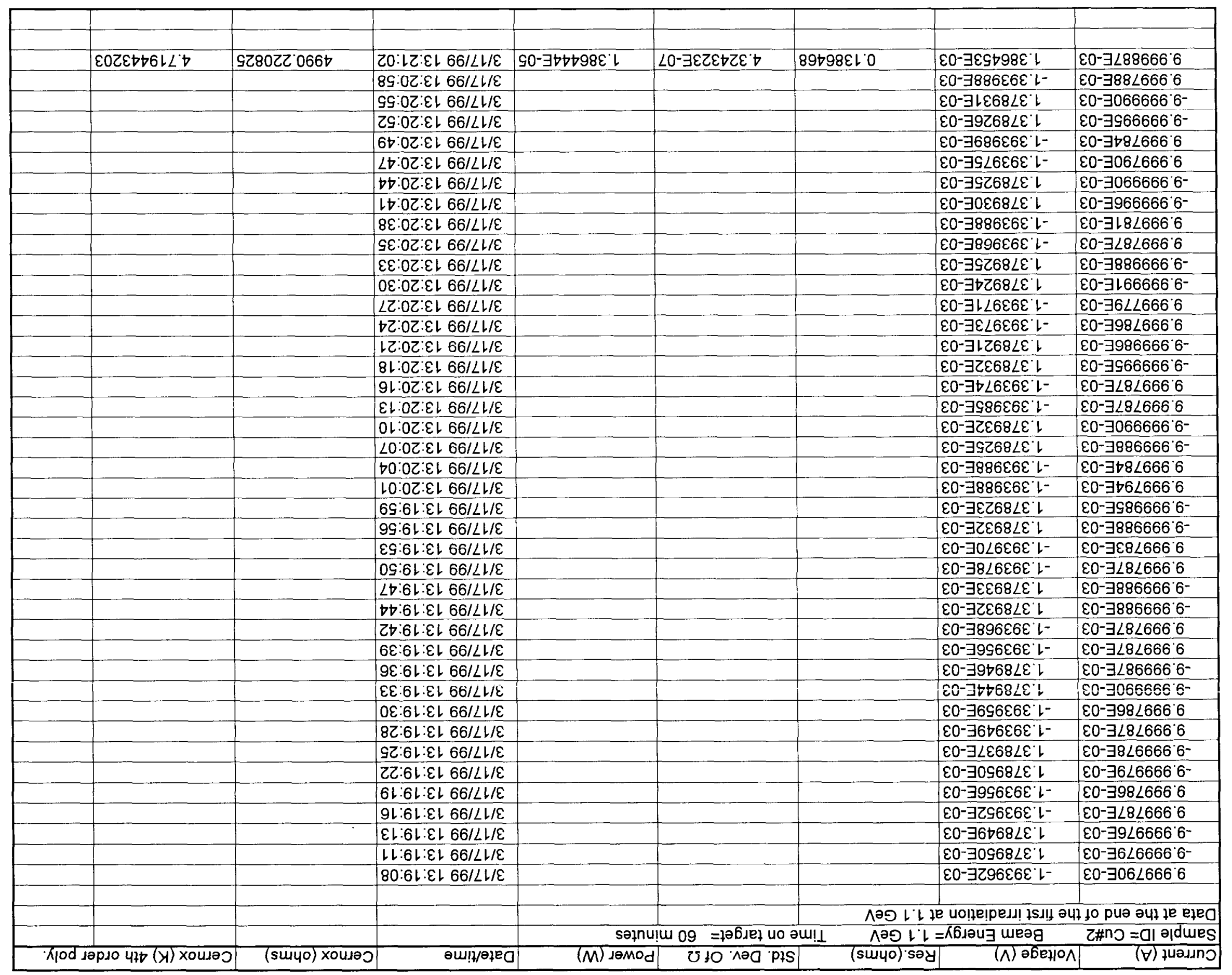


Copper2.xIs

\begin{tabular}{|c|c|c|c|c|c|c|c|c|}
\hline Current $(A)$ & Voltage (V) & Res.(ohms) & Std. Dev. Of $\Omega$ & Power (W) & Date/time & Cernox (ohms) & \multicolumn{2}{|c|}{ Cernox $(K)$ 4th order poly. } \\
\hline Sample ID= Cu\#2 & \multicolumn{2}{|c|}{ Beam Energy $=1.1 \mathrm{GeV}$} & \multicolumn{2}{|c|}{ Time on target $=60$ minutes } & & & & \\
\hline \multicolumn{9}{|c|}{ Dample ID = Cut 2} \\
\hline & & & & & & & & \\
\hline $1.499875 \mathrm{E}-02$ & $-2.087280 \mathrm{E}-03$ & & & & $3 / 17 / 9913: 21: 30$ & & & \\
\hline$-1.500277 \mathrm{E}-02$ & $2.072303 E-03$ & & & & $3 / 17 / 9913: 21: 33$ & & & \\
\hline$-1.500272 E-02$ & $2.072334 \mathrm{E}-03$ & & & & $3 / 17 / 99$ 13:21:36 & & & \\
\hline 1.499873E-02 & $-2.087270 \mathrm{E}-03$ & & & & 3/17/99 13:21:38 & & & \\
\hline 1.499875E-02 & $-2.087273 E-03$ & & & & $3 / 17 / 9913: 21: 41$ & & & \\
\hline$-1.500278 \mathrm{E}-02$ & $2.072322 \mathrm{E}-03$ & & & & $3 / 17 / 99$ 13:21:44 & & & \\
\hline$-1.500275 E-02$ & $2.072304 \mathrm{E}-03$ & & & & $3 / 17 / 99$ 13:21:47 & & & \\
\hline 1.499885E-02 & $-2.087283 \mathrm{E}-03$ & & & & $3 / 17 / 9913: 21: 50$ & & & \\
\hline 1.499891E-02 & $-2.087293 \mathrm{E}-03$ & & & & $3 / 17 / 9913: 21: 53$ & & & \\
\hline$-1.500270 \mathrm{C}-02$ & $2.072280 \mathrm{E}-03$ & & & & $3 / 17 / 99$ 13:21:55 & & & \\
\hline$-1.500265 \mathrm{E}-02$ & $2.072294 \mathrm{E}-03$ & & & & 3/17/99 13:21:58 & & & \\
\hline 1.499881E-02 & $-2.087285 E-03$ & & & & $3 / 17 / 9913: 22: 01$ & & & \\
\hline $1.499882 \mathrm{E}-02$ & $-2.087273 \mathrm{E}-03$ & & & & $3 / 17 / 9913: 22: 04$ & & & \\
\hline$-1.500274 E-02$ & $2.072308 E-03$ & & & & $3 / 17 / 9913: 22: 07$ & & & \\
\hline$-1.500274 \mathrm{E}-02$ & $2.072315 \mathrm{E}-03$ & & & & $3 / 17 / 99 \quad 13: 22: 10$ & & & \\
\hline $1.499883 \mathrm{E}-02$ & $-2.087287 \mathrm{E}-03$ & & & & $3 / 17 / 99$ 13:22:12 & & & \\
\hline $1.499887 \mathrm{E}-02$ & $-2.087310 \mathrm{E}-03$ & & & & $3 / 17 / 99$ 13:22:15 & & & \\
\hline$-1.500270 \mathrm{E}-02$ & $2.072298 \mathrm{E}-03$ & & & & $3 / 17 / 99$ 13:22:18 & & & \\
\hline$-1.500271 \mathrm{E}-02$ & $2.072306 \mathrm{E}-03$ & & & & $3 / 17 / 99$ 13:22:21 & & & \\
\hline 1.499876E-02 & $-2.087283 E-03$ & & & & $3 / 17 / 99$ 13:22:24 & & & \\
\hline $1.499880 \mathrm{E}-02$ & $-2.087298 E-03$ & & & & $3 / 17 / 99$ 13:22:26 & & & \\
\hline$-1.500270 \mathrm{E}-02$ & $2.072299 \mathrm{E}-03$ & & & & $3 / 17 / 99$ 13:22:29 & & & \\
\hline$-1.500269 \mathrm{E}-02$ & $2.072290 \mathrm{E}-03$ & & & & $3 / 17 / 99$ 13:22:32 & & & \\
\hline $1.499878 \mathrm{E}-02$ & $-2.087281 E-03$ & & & & $3 / 17 / 9913: 22: 35$ & & & \\
\hline 1.499883E-02 & $-2.087282 E-03$ & & & & $3 / 17 / 99$ 13:22:38 & & & \\
\hline$-1.500272 E-02$ & $2.072275 \mathrm{E}-03$ & & & & $3 / 17 / 9913: 22: 41$ & & & \\
\hline$-1.500270 \mathrm{E}-02$ & $2.072281 \mathrm{E}-03$ & & & & $3 / 17 / 99$ 13:22:43 & & & \\
\hline 1.499881E-02 & $-2.087308 E-03$ & & & & $3 / 17 / 99$ 13:22:46 & & & \\
\hline 1.499886E-02 & $-2.087304 \mathrm{E}-03$ & & & & $3 / 17 / 99$ 13:22:49 & & & \\
\hline$-1.500279 \mathrm{E}-02$ & $2.072301 \mathrm{E}-03$ & & & & $3 / 17 / 99$ 13:22:52 & & & \\
\hline$-1.500276 \mathrm{E}-02$ & 2.072303E-03 & & & & $3 / 17 / 99$ 13:22:55 & & & \\
\hline $1.499878 \mathrm{E}-02$ & $-2.087310 \mathrm{E}-03$ & & & & $3 / 17 / 9913: 22: 58$ & & & \\
\hline $1.499880 \mathrm{E}-02$ & $-2.087303 E-03$ & & & & $3 / 17 / 99$ 13:23:00 & & & \\
\hline$-1.500275 \mathrm{E}-02$ & $2.072268 \mathrm{E}-03$ & & & & 3/17/99 13:23:03 & & & \\
\hline$-1.500273 \mathrm{E}-02$ & $2.072279 \mathrm{E}-03$ & & & & 3/17/99 13:23:06 & & & \\
\hline $1.499885 \mathrm{E}-02$ & $-2.087323 E-03$ & & & & $3 / 17 / 99$ 13:23:09 & & & \\
\hline 1.499879E-02 & $-2.087326 \mathrm{E}-03$ & & & & $3 / 17 / 99$ 13:23:12 & & & \\
\hline$-1.500274 \mathrm{E}-02$ & $2.072276 \mathrm{E}-03$ & & & & $3 / 17 / 99$ 13:23:15 & & & \\
\hline$-1.500276 \mathrm{E}-02$ & $2.072263 \mathrm{E}-03$ & & & & 3/17/99 13:23:17 & & & \\
\hline $1.499882 \mathrm{E}-02$ & $-2.087352 E-03$ & & & & $3 / 17 / 99$ 13:23:20 & & & \\
\hline $1.500077 \mathrm{E}-02$ & $2.079796 \mathrm{E}-03$ & 0.1386459 & $5.300420 \mathrm{E}-07$ & $3.119876 \mathrm{E}-05$ & $3 / 17 / 99$ 13:23:24 & 4989.208866 & 4.719979445 & \\
\hline & & & & & & & & \\
\hline & & & & & & & & \\
\hline
\end{tabular}


Copper2.xls

\begin{tabular}{|c|c|c|c|c|c|c|c|c|}
\hline \multirow{3}{*}{\begin{tabular}{|l|} 
Current $(A)$ \\
Sample ID= Cu\#2 \\
End of second and \\
\end{tabular}} & Voltage (V) & Res.(ohms) & & & \multirow[t]{2}{*}{ Date/time } & \multirow[t]{2}{*}{ Cernox (ohms) } & \multicolumn{2}{|c|}{ Cernox $(K)$ 4th order poly. } \\
\hline & \multirow{2}{*}{\multicolumn{2}{|c|}{$\begin{array}{l}\text { Beam Energy }=1.1 \mathrm{GeV} \\
\text { last irradiation at } 1.1 \mathrm{GeV}\end{array}$}} & \begin{tabular}{c|c|} 
Std. Dev. Of $\Omega$ & Power $(W)$ \\
Time on target $=135$ minutes
\end{tabular} & & & & & \\
\hline & & & \multicolumn{6}{|c|}{ End of second and last irradiation at $1.1 \mathrm{GeV}$} \\
\hline & & & & & & & & \\
\hline 5.000185E-03 & $-7.022329 \mathrm{E}-04$ & & & & $3 / 17 / 9915: 37: 15$ & & & \\
\hline$-5.000493 \mathrm{E}-03$ & $6.873282 \mathrm{E}-04$ & & & & $3 / 17 / 9915: 37: 18$ & & & \\
\hline$-5.000495 \mathrm{E}-03$ & $6.873362 \mathrm{E}-04$ & & & & $3 / 17 / 9915: 37: 21$ & & & \\
\hline 5.000191E-03 & $-7.022188 \mathrm{E}-04$ & & & & $3 / 17 / 9915: 37: 24$ & & & \\
\hline $5.000195 \mathrm{E}-03$ & $-7.022324 E-04$ & & & & $3 / 17 / 9915: 37: 27$ & & & \\
\hline$-5.000498 \mathrm{E}-03$ & $6.873356 \mathrm{E}-04$ & & & & $3 / 17 / 9915: 37: 29$ & & & \\
\hline$-5.000498 \mathrm{E}-03$ & $6.873407 \mathrm{E}-04$ & & & & $3 / 17 / 9915: 37: 32$ & & & \\
\hline $5.000195 \mathrm{E}-03$ & $-7.022185 \mathrm{E}-04$ & & & & $3 / 17 / 9915: 37: 35$ & & & \\
\hline $5.000195 \mathrm{E}-03$ & $-7.022213 \mathrm{E}-04$ & & & & $3 / 17 / 9915: 37: 38$ & & & \\
\hline$-5.000499 \mathrm{E}-03$ & $6.873410 \mathrm{E}-04$ & & & & $3 / 17 / 9915: 37: 41$ & & & \\
\hline$-5.000497 \mathrm{E}-03$ & $6.873352 E-04$ & & & & $3 / 17 / 9915: 37: 43$ & & & \\
\hline $5.000194 \mathrm{E}-03$ & $-7.022191 \mathrm{E}-04$ & & & & $3 / 17 / 9915: 37: 46$ & & & \\
\hline $5.000196 \mathrm{E}-03$ & $-7.022146 \mathrm{E}-04$ & & & & $3 / 17 / 9915: 37: 49$ & & & \\
\hline$-5.000498 \mathrm{E}-03$ & $6.873307 \mathrm{E}-04$ & & & & $3 / 17 / 9915: 37: 52$ & & & \\
\hline$-5.000497 \mathrm{E}-03$ & $6.873377 \mathrm{E}-04$ & & & & $3 / 17 / 9915: 37: 55$ & & & \\
\hline $5.000194 \mathrm{E}-03$ & $-7.022173 \mathrm{E}-04$ & & & & $3 / 17 / 9915: 37: 58$ & & & \\
\hline $5.000197 \mathrm{E}-03$ & $-7.022214 \mathrm{E}-04$ & & & & 3/17/99 15:38:00 & & & \\
\hline$-5.000501 E-03$ & $6.873396 \mathrm{E}-04$ & & & & $3 / 17 / 99$ 15:38:03 & & & \\
\hline$-5.000502 E-03$ & $6.873401 \mathrm{E}-04$ & & & & $3 / 17 / 9915: 38: 06$ & & & \\
\hline $5.000190 \mathrm{E}-03$ & $-7.022011 E-04$ & & & & $3 / 17 / 99$ 15:38:09 & & & \\
\hline 5.000193E-03 & $-7.022087 \mathrm{E}-04$ & & & & $3 / 17 / 9915: 38: 12$ & & & \\
\hline$-5.000500 \mathrm{E}-03$ & $6.873435 \mathrm{E}-04$ & & & & $3 / 17 / 9915: 38: 15$ & & & \\
\hline$-5.000501 E-03$ & $6.873409 \mathrm{E}-04$ & & & & $3 / 17 / 9915: 38: 17$ & & & \\
\hline $5.000194 \mathrm{E}-03$ & $-7.022046 \mathrm{E}-04$ & & & & $3 / 17 / 9915: 38: 20$ & & & \\
\hline $5.000195 \mathrm{E}-03$ & $-7.022013 \mathrm{E}-04$ & & & & $3 / 17 / 9915: 38: 23$ & & & \\
\hline$-5.000503 E-03$ & $6.873439 \mathrm{E}-04$ & & & & $3 / 17 / 99$ 15:38:26 & & & \\
\hline$-5.000503 E-03$ & $6.873496 \mathrm{E}-04$ & & & & $3 / 17 / 99 \quad 15: 38: 29$ & & & \\
\hline 5.000195E-03 & $-7.022120 \mathrm{E}-04$ & & & & $3 / 17 / 99$ 15:38:32 & & & \\
\hline $5.000192 \mathrm{E}-03$ & $-7.022080 \mathrm{E}-04$ & & & & $3 / 17 / 9915: 38: 34$ & & & \\
\hline$-5.000504 E-03$ & $6.873512 \mathrm{E}-04$ & & & & 3/17/99 15:38:37 & & & \\
\hline$-5.000502 E-03$ & $6.873563 \mathrm{E}-04$ & & & & $3 / 17 / 9915: 38: 40$ & & & \\
\hline $5.000189 \mathrm{E}-03$ & $-7.022089 \mathrm{E}-04$ & & & & $3 / 17 / 99$ 15:38:43 & & & \\
\hline $5.000190 \mathrm{E}-03$ & $-7.022012 \mathrm{E}-04$ & & & & $3 / 17 / 9915: 38: 46$ & & & \\
\hline$-5.000506 \mathrm{E}-03$ & $6.873555 E-04$ & & & & $3 / 17 / 9915: 38: 49$ & & & \\
\hline$-5.000505 E-03$ & $6.873544 E-04$ & & & & $3 / 17 / 9915: 38: 51$ & & & \\
\hline $5.000188 \mathrm{E}-03$ & $-7.021907 \mathrm{E}-04$ & & & & $3 / 17 / 9915: 38: 54$ & & & \\
\hline $5.000189 \mathrm{E}-03$ & $-7.021906 \mathrm{E}-04$ & & & & $3 / 17 / 9915: 38: 57$ & & & \\
\hline$-5.000507 \mathrm{E}-03$ & $6.873474 \mathrm{E}-04$ & & & & 3/17/99 15:39:00 & & & \\
\hline$-5.000505 \mathrm{E}-03$ & $6.873524 \mathrm{E}-04$ & & & & $3 / 17 / 9915: 39: 03$ & & & \\
\hline $5.000190 \mathrm{E}-03$ & $-7.021877 \mathrm{E}-04$ & & & & 3/17/99 15:39:06 & & & \\
\hline $5.000347 \mathrm{E}-03$ & $6.947768 \mathrm{E}-04$ & 0.1389457 & $8.877651 \mathrm{E}-07$ & $3.474092 \mathrm{E}-06$ & $3 / 17 / 99$ 15:39:10 & 4977.358203 & 4.72627378 & \\
\hline & & & & & & & & \\
\hline
\end{tabular}




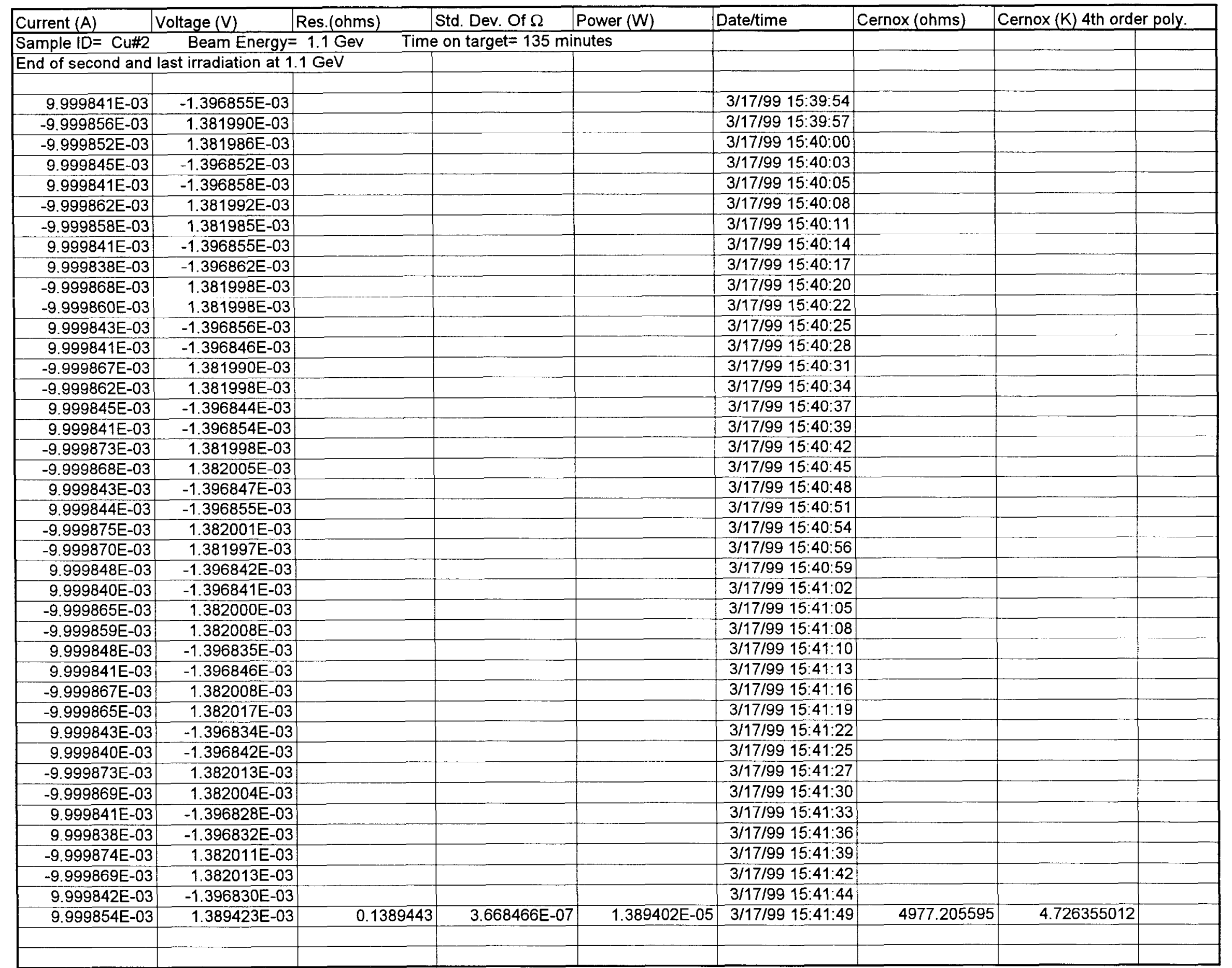




\begin{tabular}{|c|c|c|c|c|c|c|c|c|}
\hline \multirow{3}{*}{\begin{tabular}{|l|} 
Current $(A)$ \\
Sample ID $=$ Cu\#2 \\
End of second and
\end{tabular}} & Voltage (V) & Res.(ohms) & & & \multirow{2}{*}{ Date/time } & \multirow[t]{2}{*}{ Cernox (ohms) } & \multicolumn{2}{|c|}{ Cernox (K) 4th order poly } \\
\hline & \multirow{2}{*}{\multicolumn{2}{|c|}{ Beam Energy $=1.1 \mathrm{GeV}$}} & $\frac{\text { Std. Dev. Of } \Omega}{\text { Time on target }=135 \text { minutes }}$ & & & & & \\
\hline & & & & & & & & \\
\hline & & & & & & & & \\
\hline $1.499814 \mathrm{E}-02$ & $-2.091649 \mathrm{E}-03$ & & & & 3/17/99 15:42:19 & & & \\
\hline$-1.500055 \mathrm{E}-02$ & $2.076512 \mathrm{E}-03$ & & & & $3 / 17 / 9915: 42: 22$ & & & \\
\hline$-1.500053 \mathrm{E}-02$ & $2.076497 \mathrm{E}-03$ & & & & $3 / 17 / 99$ 15:42:24 & & & \\
\hline 1.499823E-02 & $-2.091674 \mathrm{E}-03$ & & & & $3 / 17 / 9915: 42: 27$ & & & \\
\hline 1.499827E-02 & $-2.091687 \mathrm{E}-03$ & & & & $3 / 17 / 9915: 42: 30$ & & & \\
\hline$-1.500054 \mathrm{E}-02$ & $2.076519 \mathrm{E}-03$ & & & & $3 / 17 / 99$ 15:42:33 & & & \\
\hline$-1.500055 \mathrm{E}-02$ & $2.076524 \mathrm{E}-03$ & & & & $3 / 17 / 9915: 42: 36$ & & & \\
\hline 1.499824E-02 & $-2.091663 E-03$ & & & & $3 / 17 / 99 \quad 15: 42: 38$ & & & \\
\hline 1.499824E-02 & $-2.091663 \mathrm{E}-03$ & & & & $3 / 17 / 9915: 42: 41$ & & & \\
\hline$-1.500055 \mathrm{E}-02$ & $2.076508 \mathrm{E}-03$ & & & & $3 / 17 / 9915: 42: 44$ & & & \\
\hline$-1.500052 E-02$ & $2.076495 \mathrm{E}-03$ & & & & $3 / 17 / 99$ 15:42:47 & & & \\
\hline $1.499832 \mathrm{E}-02$ & $-2.091670 \mathrm{E}-03$ & & & & $3 / 17 / 9915: 42: 50$ & & & \\
\hline $1.499830 \mathrm{E}-02$ & $-2.091676 \mathrm{E}-03$ & & & & $3 / 17 / 99 \quad 15: 42: 53$ & & & \\
\hline$-1.500052 \mathrm{E}-02$ & $2.076506 \mathrm{E}-03$ & & & & $3 / 17 / 9915: 42: 55$ & & & \\
\hline$-1.500049 \mathrm{E}-02$ & $2.076513 \mathrm{E}-03$ & & & & $3 / 17 / 9915: 42: 58$ & & & \\
\hline $1.499832 \mathrm{E}-02$ & $-2.091678 \mathrm{E}-03$ & & & & $3 / 17 / 9915: 43: 01$ & & & \\
\hline 1.499831E-02 & $-2.091686 \mathrm{E}-03$ & & & & $3 / 17 / 99$ 15:43:04 & & & \\
\hline$-1.500050 \mathrm{E}-02$ & $2.076492 E-03$ & & & & $3 / 17 / 9915: 43: 07$ & & & \\
\hline$-1.500054 \mathrm{E}-02$ & $2.076505 E-03$ & & & & $3 / 17 / 99$ 15:43:10 & & & \\
\hline 1.499832E-02 & $-2.091688 \mathrm{E}-03$ & & & & $3 / 17 / 99$ 15:43:12 & & & \\
\hline $1.499835 \mathrm{E}-02$ & $-2.091682 E-03$ & & & & 3/17/99 15:43:15 & & & \\
\hline$-1.500063 \mathrm{E}-02$ & $2.076521 \mathrm{E}-03$ & & & & $3 / 17 / 9915: 43: 18$ & & & \\
\hline$-1.500059 \mathrm{E}-02$ & $2.076516 \mathrm{E}-03$ & & & & $3 / 17 / 99$ 15:43:21 & & & \\
\hline 1.499833E-02 & $-2.091679 \mathrm{E}-03$ & & & & $3 / 17 / 99$ 15:43:24 & & & \\
\hline $1.499833 E-02$ & $-2.091678 \mathrm{E}-03$ & & & & $3 / 17 / 99$ 15:43:26 & & & \\
\hline$-1.500059 \mathrm{E}-02$ & $2.076506 \mathrm{E}-03$ & & & & $3 / 17 / 9915: 43: 29$ & & & \\
\hline$-1.500060 \mathrm{E}-02$ & $2.076515 \mathrm{E}-03$ & & & & $3 / 17 / 99$ 15:43:32 & & & \\
\hline 1.499829E-02 & $-2.091677 \mathrm{E}-03$ & & & & $3 / 17 / 99$ 15:43:35 & & & \\
\hline 1.499828E-02 & $-2.091674 \mathrm{E}-03$ & & & & $3 / 17 / 99$ 15:43:38 & & & \\
\hline$-1.500062 E-02$ & $2.076525 E-03$ & & & & $3 / 17 / 9915: 43: 41$ & & & \\
\hline$-1.500065 E-02$ & $2.076517 E-03$ & & & & $3 / 17 / 99$ 15:43:44 & & & \\
\hline 1.499826E-02 & $-2.091673 E-03$ & & & & $3 / 17 / 99$ 15:43:46 & & & \\
\hline $1.499828 E-02$ & $-2.091674 \mathrm{E}-03$ & & & & $3 / 17 / 9915: 43: 49$ & & & \\
\hline$-1.500064 E-02$ & $2.076525 \mathrm{E}-03$ & & & & $3 / 17 / 99$ 15:43:52 & & & \\
\hline$-1.500063 E-02$ & $2.076535 E-03$ & & & & $3 / 17 / 99$ 15:43:55 & & & \\
\hline $1.499825 E-02$ & $-2.091648 \mathrm{E}-03$ & & . & & $3 / 17 / 99$ 15:43:58 & & & \\
\hline $1.499829 E-02$ & $-2.091634 \mathrm{E}-03$ & & & & $3 / 17 / 9915: 44: 00$ & & & \\
\hline$-1.500062 \mathrm{E}-02$ & $2.076547 \mathrm{E}-03$ & & & & $3 / 17 / 99$ 15:44:03 & & & \\
\hline$-1.500066 \mathrm{E}-02$ & $2.076572 \mathrm{E}-03$ & & & & $3 / 17 / 9915: 44: 06$ & & & \\
\hline $1.499826 \mathrm{E}-02$ & $-2.091608 E-03$ & & & & $3 / 17 / 99$ 15:44:09 & & & \\
\hline 1.499943E-02 & $2.084093 E-03$ & 0.1389448 & 3.322316E-07 & $3.126022 \mathrm{E}-05$ & $3 / 17 / 99$ 15:44:13 & 4976.698551 & 4.72662494 & \\
\hline & & & & & & & & \\
\hline & & & & & & & & \\
\hline
\end{tabular}




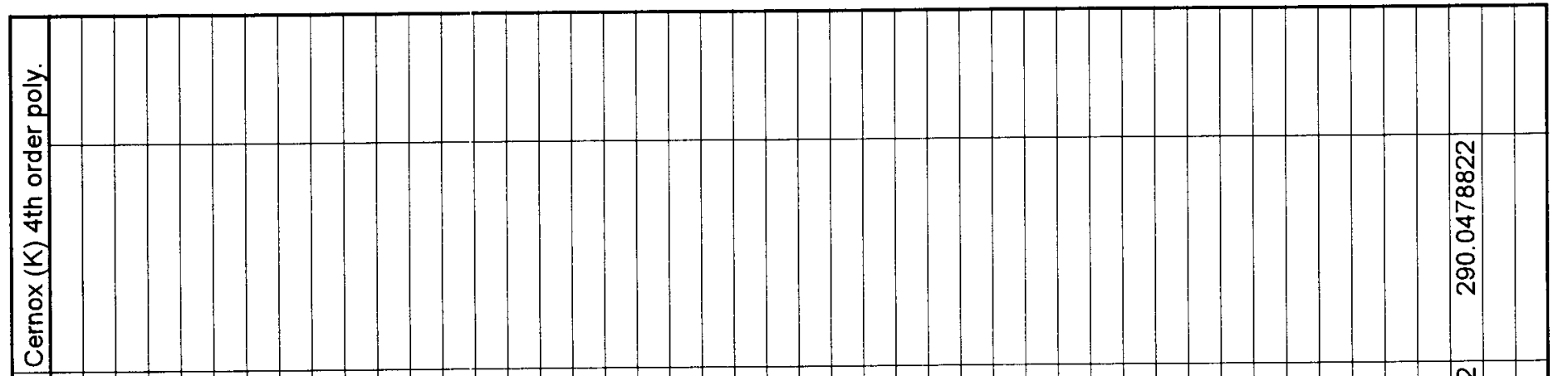

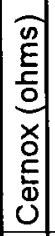

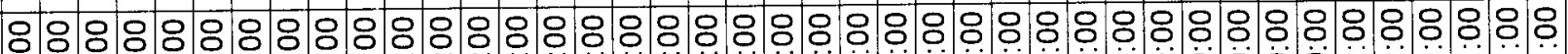

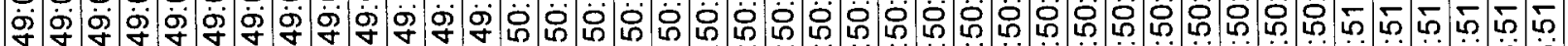

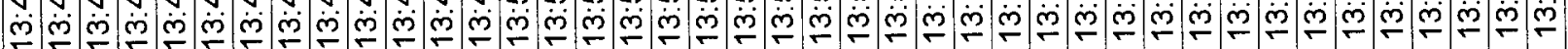

: $\quad$ இ

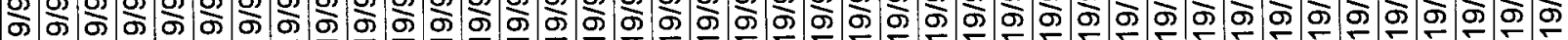

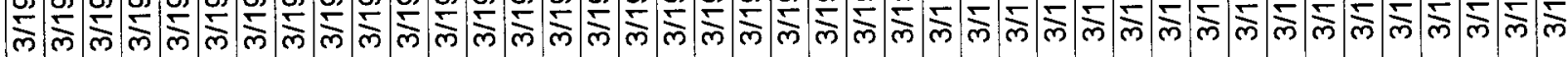

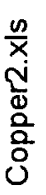

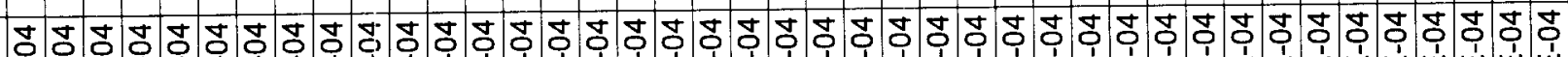

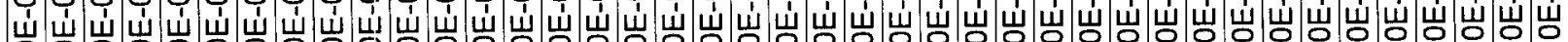

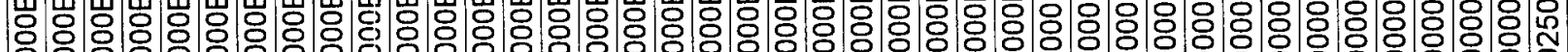

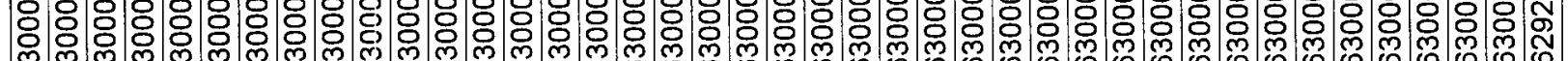
ర్లు

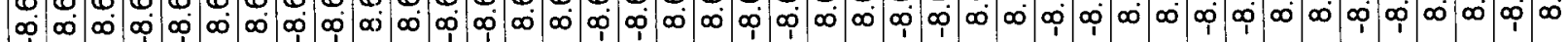

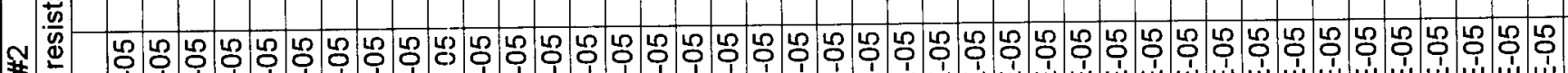

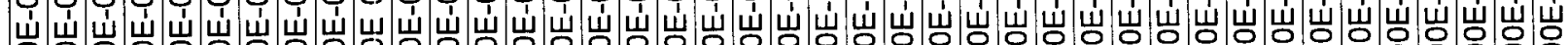

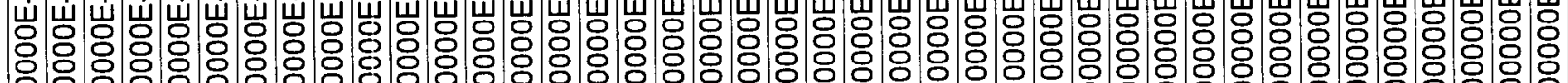
- 
Copper2.xis

\begin{tabular}{|c|c|c|c|c|c|c|c|c|}
\hline Current (A) & Voltage (V) & Res.(ohms) & Std. Dev. Of $\Omega$ & Power (W) & Date/time & Cernox (ohms) & Cernox $(K)$ 4th orde & ar poly. \\
\hline Sample ID= Cu\#2 & Beam Energy $=$ & $0 \quad$ Time on tars & get $=0$ & & & & & \\
\hline Post irradiation resi & istance measuremer & ents at ambient tem & perature in the U-lin & & & & & \\
\hline & & & & & & & & \\
\hline $5.000000 \mathrm{E}-04$ & $-8.630000 \mathrm{E}-03$ & & & & $3 / 19 / 99$ 13:51:00 & & & \\
\hline$-5.000000 \mathrm{E}-04$ & $8.630000 \mathrm{E}-03$ & & & & 3/19/99 13:51:00 & & & \\
\hline$-5.000000 \mathrm{E}-04$ & $8.630000 \mathrm{E}-03$ & & & & $3 / 19 / 99$ 13:51:00 & & & \\
\hline $5.000000 \mathrm{E}-04$ & $-8.630000 \mathrm{E}-03$ & & & & $3 / 19 / 9913: 51: 00$ & & & \\
\hline $5.000000 \mathrm{E}-04$ & $-8.630000 \mathrm{E}-03$ & & & & $3 / 19 / 99$ 13:51:00 & & & \\
\hline$-5.000000 \mathrm{E}-04$ & $8.630000 \mathrm{E}-03$ & & & & $3 / 19 / 9913: 51: 00$ & & & \\
\hline$-5.000000 \mathrm{E}-04$ & $8.630000 \mathrm{E}-03$ & & & & $3 / 19 / 99$ 13:52:00 & & & \\
\hline $5.000000 \mathrm{E}-04$ & $-8.630000 \mathrm{E}-03$ & & & & $3 / 19 / 99$ 13:52:00 & & & \\
\hline $5.000000 \mathrm{E}-04$ & $-8.630000 \mathrm{E}-03$ & & & & $3 / 19 / 99$ 13:52:00 & & & \\
\hline$-5.0000000 E-044$ & $8.630000 E-03$ & & & & $3 / 19 / 9913: 52: 00$ & & & \\
\hline$-5.000000 \mathrm{E}-04$ & $8.630000 \mathrm{E}-03$ & & & & $3 / 19 / 99 \quad 13: 52: 00$ & & & \\
\hline $5.000000 \mathrm{E}-04$ & $-8.630000 \mathrm{E}-03$ & & & & 3/19/99 13:52:00 & & & \\
\hline $5.000000 \mathrm{E}-04$ & $-8.630000 \mathrm{E}-03$ & & & & $3 / 19 / 99 \quad 13: 52: 00$ & & & \\
\hline$-5.000000 E-04$ & $8.630000 \mathrm{E}-03$ & & & & $3 / 19 / 99$ 13:52:00 & & & \\
\hline$-5.000000 \mathrm{E}-04$ & $8.630000 \mathrm{E}-03$ & & & & $3 / 19 / 9913: 52: 00$ & & & \\
\hline $5.000000 \mathrm{E}-04$ & $-8.630000 \mathrm{E}-03$ & & & & $3 / 19 / 9913: 52: 00$ & & & \\
\hline $5.000000 \mathrm{E}-04$ & $-8.630000 \mathrm{E}-03$ & & & & $3 / 19 / 99$ 13:52:00 & & & \\
\hline-5.000000 E- 04 & $8.630000 \mathrm{E}-03$ & & & & $3 / 19 / 99 \quad 13: 52: 00$ & & & \\
\hline$-5.000000 E-04$ & $8.630000 \mathrm{E}-03$ & & & & $3 / 19 / 9913: 52: 00$ & & & \\
\hline $5.000000 \mathrm{E}-04$ & $-8.630000 \mathrm{E}-03$ & & & & $3 / 19 / 9913: 52: 00$ & & & \\
\hline $5.000000 \mathrm{E}-04$ & $-8.630000 \mathrm{E}-03$ & & & & $3 / 19 / 99$ 13:52:00 & & & \\
\hline$-5.000000 \mathrm{E}-04$ & $8.630000 \mathrm{E}-03$ & & & & $3 / 19 / 99$ 13:52:00 & & & \\
\hline$-5.000000 \mathrm{E}-04$ & $8.630000 E-03$ & & & & $3 / 19 / 9913: 52: 00$ & & & \\
\hline $5.000000 \mathrm{E}-04$ & $-8.630000 \mathrm{E}-03$ & & & & 3/19/99 13:52:00 & & & \\
\hline $5.000000 \mathrm{E}-04$ & $-8.630000 \mathrm{E}-03$ & & & & $3 / 19 / 99$ 13:52:00 & & & \\
\hline$-5.000000 \mathrm{E}-04$ & $8.630000 \mathrm{E}-03$ & & & & $3 / 19 / 9913: 52: 00$ & & & \\
\hline$-5.000000 \mathrm{E}-04$ & $8.630000 \mathrm{E}-03$ & & & & $3 / 19 / 9913: 52: 00$ & & & \\
\hline $5.000000 \mathrm{E}-04$ & $-8.630000 E-03$ & & & & $3 / 19 / 99$ 13:53:00 & & & \\
\hline $5.000000 \mathrm{E}-04$ & $-8.630000 \mathrm{E}-03$ & & & & $3 / 19 / 99$ 13:53:00 & & & \\
\hline$-5.000000 \mathrm{E}-04$ & 8.630000 E-03 & & & & $3 / 19 / 99$ 13:53:00 & & & \\
\hline$-5.000000 \mathrm{E}-04$ & $8.630000 E-03$ & & & & $3 / 19 / 99$ 13:53:00 & & & \\
\hline $5.000000 \mathrm{E}-04$ & $-8.630000 E-03$ & & & & $3 / 19 / 99$ 13:53:00 & & & \\
\hline 5.000000 E- 04 & $-8.630000 E-03$ & & & & 3/19/99 13:53:00 & & & \\
\hline$-5.000000 \mathrm{E}-04$ & $8.630000 E-03$ & & & & 3/19/99 13:53:00 & & & \\
\hline$-5.000000 \mathrm{E}-04$ & $8.630000 \mathrm{E}-03$ & & & & 3/19/99 13:53:00 & & & \\
\hline $5.000000 \mathrm{E}-04$ & $-8.630000 \mathrm{E}-03$ & & & & $3 / 19 / 99$ 13:53:00 & & & \\
\hline $5.000000 \mathrm{E}-04$ & $-8.630000 \mathrm{E}-03$ & & & & 3/19/99 13:53:00 & & & \\
\hline$-5.000000 \mathrm{E}-04$ & $8.630000 \mathrm{E}-03$ & & & & $3 / 19 / 99$ 13:53:00 & & & \\
\hline$-5.000000 \bar{E}-04$ & $8.630000 \mathrm{E}-03$ & & & & $3 / 19 / 99$ 13:53:00 & & & \\
\hline $5.000000 \mathrm{E}-04$ & $-8.630000 \mathrm{E}-03$ & & & & $3 / 19 / 99$ 13:53:00 & & & \\
\hline $5.000000 \mathrm{E}-04$ & $8.628456 \mathrm{E}-03$ & 17.2555178 & $8.290000 \mathrm{E}-05$ & $4.310000 \mathrm{E}-06$ & $3 / 19 / 99$ 13:53:00 & 60.66026644 & 290.029859 & \\
\hline
\end{tabular}


COPPER2.xls

\begin{tabular}{|c|c|c|c|c|c|c|c|c|}
\hline Current $(\mathrm{A})$ & Voltage (V) & Res.(ohms) & Std. Dev. Of $\Omega$ & Power (W) & \multirow[t]{2}{*}{ Date/time } & \multirow[t]{2}{*}{ Cernox (ohms) } & \multicolumn{2}{|c|}{ Cernox $(\mathrm{K})$ 4th order poly } \\
\hline Sample ID=Cu2 & \multicolumn{4}{|c|}{ Beam Energy $=1.1 \mathrm{GeV} \quad$ Time on target $=135$ minutes } & & & & \\
\hline \multicolumn{9}{|c|}{ post-irradiation cooldown data after $1.1 \mathrm{GeV}$ runs and after 3-month room temperature anneal } \\
\hline & & & & & & & & \\
\hline $5.000385 E-03$ & $-6.997061 \mathrm{E}-04$ & & & & $6 / 22 / 9915: 54: 26$ & & & \\
\hline$-5.001007 \mathrm{E}-03$ & $6.831721 E-04$ & & & & $6 / 22 / 9915: 54: 29$ & & & \\
\hline$-5.001002 E-03$ & $6.831689 \mathrm{E}-04$ & & & & $6 / 22 / 9915: 54: 32$ & & & \\
\hline $5.000392 E-03$ & $-6.996979 \mathrm{E}-04$ & & & & $6 / 22 / 9915: 54: 35$ & & & \\
\hline $5.000388 \mathrm{E}-03$ & $-6.996996 \mathrm{E}-04$ & & & & $6 / 22 / 9915: 54: 38$ & & & \\
\hline$-5.001017 \mathrm{E}-03$ & $6.832168 \mathrm{E}-04$ & & & & $6 / 22 / 9915: 54: 40$ & & & \\
\hline$-5.001006 \mathrm{E}-03$ & $6.831957 \mathrm{E}-04$ & & & & $6 / 22 / 9915: 54: 43$ & & & \\
\hline $5.000391 \mathrm{E}-03$ & $-6.996790 \mathrm{E}-04$ & & & & $6 / 22 / 9915: 54: 46$ & & & \\
\hline $5.000396 \mathrm{E}-03$ & $-6.996665 \mathrm{E}-04$ & & & & $6 / 22 / 9915: 54: 49$ & & & \\
\hline$-5.001015 E-03$ & $6.832199 \mathrm{E}-04$ & & & & $6 / 22 / 9915: 54: 52$ & & & \\
\hline$-5.001003 E-03$ & 6.832273E-04 & & & & $6 / 22 / 9915: 54: 55$ & & & \\
\hline $5.000398 \mathrm{E}-03$ & $-6.996771 \mathrm{E}-04$ & & & & $6 / 22 / 9915: 54: 57$ & & & \\
\hline $5.000399 \mathrm{E}-03$ & $-6.996827 E-04$ & & & & $6 / 22 / 9915: 55: 00$ & & & \\
\hline$-5.001007 E-03$ & $6.832322 \mathrm{E}-04$ & & & & $6 / 22 / 9915: 55: 03$ & & & \\
\hline$-5.001002 E-03$ & $6.832330 \mathrm{E}-04$ & & & & $6 / 22 / 9915: 55: 06$ & & & \\
\hline $5.000398 \mathrm{E}-03$ & $-6.996454 E-04$ & & & & $6 / 22 / 9915: 55: 09$ & & & \\
\hline $5.000398 \mathrm{E}-03$ & $-6.996767 \mathrm{E}-04$ & & & & $6 / 22 / 9915: 55: 12$ & & & \\
\hline$-5.001007 E-03$ & $6.832476 \mathrm{E}-04$ & & & & $6 / 22 / 99$ 15:55:14 & & & \\
\hline$-5.001009 E-03$ & $6.832255 \mathrm{E}-04$ & & & & $6 / 22 / 9915: 55: 17$ & & & \\
\hline $5.000398 \mathrm{E}-03$ & $-6.996553 \mathrm{E}-04$ & & & & $6 / 22 / 9915: 55: 20$ & & & \\
\hline 5.000402E-03 & $-6.996552 \mathrm{E}-04$ & & & & $6 / 22 / 9915: 55: 23$ & & & \\
\hline$-5.001017 \mathrm{E}-03$ & $6.832439 \mathrm{E}-04$ & & & & $6 / 22 / 9915: 55: 26$ & & & \\
\hline$-5.001010 \mathrm{E}-03$ & 6.832491E-04 & & & & $6 / 22 / 9915: 55: 28$ & & & \\
\hline $5.000400 \mathrm{E}-03$ & $-6.996537 E-04$ & & & & $6 / 22 / 9915: 55: 31$ & & & \\
\hline $5.000402 \mathrm{E}-03$ & $-6.996671 E-04$ & & & & $6 / 22 / 9915: 55: 34$ & & & \\
\hline$-5.001009 E-03$ & $6.832483 \mathrm{E}-04$ & & & & $6 / 22 / 9915: 55: 37$ & & & \\
\hline$-5.001007 E-03$ & $6.832442 E-04$ & & & & $6 / 22 / 9915: 55: 40$ & & & \\
\hline $5.000395 E-03$ & $-6.996555 E-04$ & & & & $6 / 22 / 9915: 55: 43$ & & & \\
\hline $5.000391 \mathrm{E}-03$ & $-6.996502 \mathrm{E}-04$ & & & & $6 / 22 / 9915: 55: 45$ & & & \\
\hline$-5.001016 \mathrm{E}-03$ & $6.832472 \mathrm{E}-04$ & & & & $6 / 22 / 99$ 15:55:48 & & & \\
\hline$-5.001010 \mathrm{E}-03$ & $6.832278 \mathrm{E}-04$ & & & & $6 / 22 / 9915: 55: 51$ & & & \\
\hline $5.000392 \mathrm{E}-03$ & $-6.996768 \mathrm{E}-04$ & & & & $6 / 22 / 9915: 55: 54$ & & & \\
\hline $5.000388 \mathrm{E}-03$ & $-6.996576 \mathrm{E}-04$ & & & & $6 / 22 / 9915: 55: 57$ & & & \\
\hline$-5.001015 E-03$ & $6.832341 \mathrm{E}-04$ & & & & $6 / 22 / 9915: 56: 00$ & & & \\
\hline$-5.001015 E-03$ & $6.832442 \mathrm{E}-04$ & & & & $6 / 22 / 99$ 15:56:02 & & & \\
\hline 5.000397E-03 & $-6.996549 \mathrm{E}-04$ & & & & $6 / 22 / 9915: 56: 05$ & & & \\
\hline $5.000399 \mathrm{E}-03$ & $-6.996719 E-04$ & & & & $6 / 22 / 9915: 56: 08$ & & & \\
\hline$-5.001013 \mathrm{E}-03$ & $6.832179 \mathrm{E}-04$ & & & & $6 / 22 / 99$ 15:56:11 & & & \\
\hline$-5.001017 E-03$ & $6.832360 \mathrm{E}-04$ & & & & $6 / 22 / 9915: 56: 14$ & & & \\
\hline $5.000397 \mathrm{E}-03$ & $-6.996597 \mathrm{E}-04$ & & & & $6 / 22 / 9915: 56: 17$ & & & \\
\hline 5.000703E-03 & $6.914480 \mathrm{E}-04$ & 0.1382702 & 1.459505E-06 & $3.457728 \mathrm{E}-06$ & $6 / 22 / 99$ 15:56:21 & 4791.712063 & 4.828534525 & \\
\hline & & & & & & & & \\
\hline
\end{tabular}


COPPER2.xls

\begin{tabular}{|c|c|c|c|c|c|c|c|c|}
\hline \multirow{2}{*}{$\begin{array}{l}\text { Current }(A) \\
\text { Sample } 1 D=\text { Cu2 }\end{array}$} & Voltage (V) & Res.(ohms) & \multirow{2}{*}{ Std. Dev. Of $\Omega$} & \multirow{2}{*}{$\frac{\text { Power }(W)}{\text { nutes }}$} & \multirow[t]{2}{*}{ Date/time } & \multirow[t]{2}{*}{ Cernox (ohms) } & \multicolumn{2}{|c|}{ Cernox $(K)$ 4th order poly. } \\
\hline & Beam Energy $=1$ & $1.1 \mathrm{GeV}$ & & & & & & \\
\hline \multicolumn{9}{|c|}{ post-irradiation cooldown data after $1.1 \mathrm{GeV}$ runs and after 3 -month room temperature anneal } \\
\hline & & & & & & & & \\
\hline $9.999965 \mathrm{E}-03$ & $-1.390990 \mathrm{E}-03$ & & & & $6 / 22 / 9915: 57: 48$ & & & \\
\hline$-1.000027 \mathrm{E}-02$ & 1.374464E-03 & & & & $6 / 22 / 9915: 57: 51$ & & & \\
\hline$-1.000027 \mathrm{E}-02$ & $1.374456 E-03$ & & & & $6 / 22 / 9915: 57: 54$ & & & \\
\hline 9.999964E-03 & $-1.390971 \mathrm{E}-03$ & & & & $6 / 22 / 9915: 57: 57$ & & & \\
\hline $9.999961 \mathrm{E}-03$ & $-1.390984 \mathrm{E}-03$ & & & & $6 / 22 / 9915: 58: 00$ & & & \\
\hline$-1.000027 \mathrm{E}-02$ & $1.374464 \mathrm{E}-03$ & & & & $6 / 22 / 99$ 15:58:03 & & & \\
\hline$-1.000027 \mathrm{E}-02$ & $1.374462 \mathrm{E}-03$ & & & & $6 / 22 / 99$ 15:58:05 & & & \\
\hline $9.999975 \mathrm{E}-03$ & $-1.390933 \mathrm{E}-03$ & & & & $6 / 22 / 9915: 58: 08$ & & & \\
\hline $9.999977 \mathrm{E}-03$ & $-1.390960 \mathrm{E}-03$ & & & & $6 / 22 / 9915: 58: 11$ & & & \\
\hline$-1.000028 \mathrm{E}-02$ & $1.374475 \mathrm{E}-03$ & & & & $6 / 22 / 9915: 58: 14$ & & & \\
\hline$-1.000027 \mathrm{E}-02$ & $1.374489 E-03$ & & & & $6 / 22 / 99 \quad 15: 58: 17$ & & & \\
\hline $9.999972 \mathrm{E}-03$ & $-1.390951 E-03$ & & & & $6 / 22 / 9915: 58: 20$ & & & \\
\hline $9.999970 \mathrm{E}-03$ & $-1.390925 E-03$ & & & & $6 / 22 / 9915: 58: 22$ & & & \\
\hline$-1.000028 \mathrm{E}-02$ & $1.374498 \mathrm{E}-03$ & & & & $6 / 22 / 9915: 58: 25$ & & & \\
\hline$-1.000028 \mathrm{E}-02$ & $1.374512 E-03$ & & & & $6 / 22 / 99$ 15:58:28 & & & \\
\hline 9.999972E-03 & $-1.390926 E-03$ & & & & $6 / 22 / 99$ 15:58:31 & & & \\
\hline $9.999964 \mathrm{E}-03$ & $-1.390936 \mathrm{E}-03$ & & & & $6 / 22 / 9915: 58: 34$ & & & \\
\hline$-1.000028 E-02$ & $1.374529 \mathrm{E}-03$ & & & & $6 / 22 / 9915: 58: 37$ & & & \\
\hline$-1.000027 \mathrm{E}-02$ & $1.374504 \mathrm{E}-03$ & & & & $6 / 22 / 9915: 58: 39$ & & & \\
\hline 9.999974E-03 & $-1.390937 \mathrm{E}-03$ & & & & $6 / 22 / 9915: 58: 42$ & & & \\
\hline $9.999968 \mathrm{E}-03$ & $-1.390934 \mathrm{E}-03$ & & & & $6 / 22 / 9915: 58: 45$ & & & \\
\hline$-1.000027 \mathrm{E}-02$ & 1.374487E-03 & & & & $6 / 22 / 9915: 58: 48$ & & & \\
\hline$-1.000027 \mathrm{E}-02$ & $1.374500 \mathrm{E}-03$ & & & & $6 / 22 / 9915: 58: 51$ & & & \\
\hline $9.999963 \mathrm{E}-03$ & $-1.390943 \mathrm{E}-03$ & & & & $6 / 22 / 9915: 58: 54$ & & & \\
\hline $9.999964 \mathrm{E}-03$ & $-1.390921 E-03$ & & & & $6 / 22 / 99$ 15:58:56 & & & \\
\hline$-1.000028 \mathrm{E}-02$ & $1.374515 E-03$ & & & & $6 / 22 / 9915: 58: 59$ & & & \\
\hline$-1.000027 \mathrm{E}-02$ & $1.374492 \mathrm{E}-03$ & & & & $6 / 22 / 9915: 59: 02$ & & & \\
\hline $9.999965 \mathrm{E}-03$ & $-1.390926 \mathrm{E}-03$ & & & & $6 / 22 / 9915: 59: 05$ & & & \\
\hline 9.999966E-03 & $-1.390930 \mathrm{E}-03$ & & & & $6 / 22 / 9915: 59: 08$ & & & \\
\hline$-1.000028 \mathrm{E}-02$ & $1.374512 \mathrm{E}-03$ & & & & $6 / 22 / 9915: 59: 10$ & & & \\
\hline$-1.000027 \mathrm{E}-02$ & 1.374514E-03 & & & & $6 / 22 / 9915: 59: 13$ & & & \\
\hline 9.999972E-03 & $-1.390933 \mathrm{E}-03$ & & & & $6 / 22 / 9915: 59: 16$ & & & \\
\hline $9.999965 \mathrm{E}-03$ & $-1.390924 \mathrm{E}-03$ & & & & $6 / 22 / 9915: 59: 19$ & & & \\
\hline$-1.000027 \mathrm{E}-02$ & $1.374497 \mathrm{E}-03$ & & & & $6 / 22 / 9915: 59: 22$ & & & \\
\hline$-1.000026 \mathrm{E}-02$ & $1.374513 \mathrm{E}-03$ & & & & $6 / 22 / 9915: 59: 25$ & & & \\
\hline $9.999974 \mathrm{E}-03$ & $-1.390934 E-03$ & & & & $6 / 22 / 9915: 59: 27$ & & & \\
\hline $9.999969 \mathrm{E}-03$ & $-1.390929 \mathrm{E}-03$ & & & & $6 / 22 / 9915: 59: 30$ & & & \\
\hline$-1.000028 \mathrm{E}-02$ & $1.374511 \mathrm{E}-03$ & & & & $6 / 22 / 9915: 59: 33$ & & & \\
\hline$-1.000028 \mathrm{E}-02$ & 1.374499E-03 & & & & $6 / 22 / 99$ 15:59:36 & & & \\
\hline $9.999969 \mathrm{E}-03$ & $-1.390924 \mathrm{E}-03$ & & & & $6 / 22 / 9915: 59: 39$ & & & \\
\hline $1.000012 \mathrm{E}-02$ & $1.382718 \mathrm{E}-03$ & 0.1382701 & $7.504761 \mathrm{E}-07$ & 1.382729E-05 & $6 / 22 / 9915: 59: 43$ & 4791.834145 & 4.828464928 & \\
\hline & & & & & & & & \\
\hline
\end{tabular}


COPPER2.xls

\begin{tabular}{|c|c|c|c|c|c|c|c|c|}
\hline \begin{tabular}{|l|} 
Current $(\mathrm{A})$ \\
Sample ID=Cu2 \\
post-irradiation coc
\end{tabular} & $\frac{\text { Voltage }(V)}{\text { Beam Energy }}=$ & Res.(ohms) & \multicolumn{2}{|c|}{\begin{tabular}{l|l} 
Std. Dev. Of $\Omega$ & Power $(W)$ \\
on target $=135$ minutes
\end{tabular}} & Date/time & Cernox (ohms) & \multicolumn{2}{|c|}{ Cernox $(K)$ 4th order poly. } \\
\hline & & & & & & & & \\
\hline $1.500141 \mathrm{E}-02$ & $-2.082530 \mathrm{E}-03$ & & & & 6/22/99 16:01:46 & & & \\
\hline$-1.500662 \mathrm{E}-02$ & $2.066645 \mathrm{E}-03$ & & & & $6 / 22 / 9916: 01: 52$ & & & \\
\hline $1.500145 \mathrm{E}-02$ & $-2.082534 E-03$ & & & & $6 / 22 / 99$ 16:01:54 & & & \\
\hline $1.500147 \mathrm{E}-02$ & $-2.082506 \mathrm{E}-03$ & & & & $6 / 22 / 9916: 01: 57$ & & & \\
\hline \begin{tabular}{|l|}
$-1.500656 E-02$ \\
\end{tabular} & $2.066678 \mathrm{E}-03$ & & & & $6 / 22 / 9916: 02: 00$ & & & \\
\hline $1.500150 \mathrm{E}-02$ & $-2.082484 E-03$ & & & & 6/22/99 16:02:08 & & & \\
\hline$-1.500663 E-02$ & $2.066708 \mathrm{E}-03$ & & & & $6 / 22 / 9916: 02: 11$ & & & \\
\hline$-1.500661 \mathrm{E}-02$ & $2.066728 \mathrm{E}-03$ & & & & $6 / 22 / 99$ 16:02:14 & & & \\
\hline $1.500145 \mathrm{E}-02$ & $-2.082449 \mathrm{E}-03$ & & & & 6/22/99 16:02:17 & & & \\
\hline $1.500144 \mathrm{E}-02$ & $-2.082463 E-03$ & & & & $6 / 22 / 99$ 16:02:20 & & & \\
\hline$-1.500667 \mathrm{E}-02$ & $2.066714 \mathrm{E}-03$ & & & & $6 / 22 / 99$ 16:02:23 & & & \\
\hline$-1.500666 \mathrm{E}-02$ & $2.066728 \mathrm{E}-03$ & & & & $6 / 22 / 99$ 16:02:25 & & & \\
\hline $1.500150 \mathrm{E}-02$ & $-2.082429 \mathrm{E}-03$ & & & & $6 / 22 / 99$ 16:02:42 & & & \\
\hline$-1.500663 \mathrm{E}-02$ & $2.066721 \mathrm{E}-03$ & & & & $6 / 22 / 99$ 16:02:45 & & & \\
\hline$-1.500659 \mathrm{E}-02$ & $2.066735 \mathrm{E}-03$ & & & & $6 / 22 / 99$ 16:02:48 & & & \\
\hline $1.500141 \mathrm{E}-02$ & $-2.082444 \mathrm{E}-03$ & & & & $6 / 22 / 9916: 02: 51$ & & & \\
\hline $1.500145 \mathrm{E}-02$ & $-2.082453 \mathrm{E}-03$ & & & & $6 / 22 / 9916: 02: 54$ & & & \\
\hline$-1.500660 \mathrm{E}-02$ & $2.066727 \mathrm{E}-03$ & & & & $6 / 22 / 99$ 16:02:57 & & & \\
\hline$-1.500666 \mathrm{E}-02$ & $2.066717 \mathrm{E}-03$ & & & & $6 / 22 / 99$ 16:02:59 & & & \\
\hline $1.500146 \mathrm{E}-02$ & $-2.082448 E-03$ & & & & $6 / 22 / 99$ 16:03:02 & & & \\
\hline $1.500149 \mathrm{E}-02$ & $-2.082463 E-03$ & & & & $6 / 22 / 99$ 16:03:05 & & & \\
\hline$-1.500666 \mathrm{E}-02$ & $2.066697 \mathrm{E}-03$ & & & & 6/22/99 16:03:08 & & & \\
\hline$-1.500661 \mathrm{E}-02$ & $2.066732 \mathrm{E}-03$ & & & & $6 / 22 / 99$ 16:03:11 & & & \\
\hline $1.500141 \mathrm{E}-02$ & $-2.082452 \mathrm{E}-03$ & & & & $6 / 22 / 99$ 16:03:13 & & & \\
\hline $1.500147 \mathrm{E}-02$ & $-2.082461 \mathrm{E}-03$ & & & & 6/22/99 16:03:16 & & & \\
\hline$-1.500660 \mathrm{E}-02$ & $2.066709 \mathrm{E}-03$ & & & & $6 / 22 / 9916: 03: 19$ & & & \\
\hline
\end{tabular}


APPENDIX 4

EXCEL DATA FILE OF RESISTANCE MEASUREMENTS OF TUNGSTEN SAMPLE 
Tungsten.xls

\begin{tabular}{|c|c|c|c|c|c|c|c|c|}
\hline \multirow{2}{*}{\begin{tabular}{|l|} 
Current $(A)$ \\
Sample ID $=W$ \\
\end{tabular}} & Voltage (V) & Res.(ohms) & \multirow{2}{*}{$\begin{array}{l}\text { Std. Dev. Of } \Omega \\
\text { et }=0\end{array}$} & \multirow[t]{2}{*}{ Power (W) } & \multirow[t]{2}{*}{ Date/time } & \multirow[t]{2}{*}{ Cernox (ohms) } & \multicolumn{2}{|c|}{ Cernox (K) 4th order poly. } \\
\hline & Beam Energy $=0$ & Time on target & & & & & & \\
\hline \multicolumn{9}{|c|}{ Pre-irradiation resistivity data at room temperature prior to testing at $1.94 \mathrm{GeV}$} \\
\hline & & & & & & & & \\
\hline $5.000260 \mathrm{E}-05$ & $-2.999824 \mathrm{E}-03$ & & & & $3 / 16 / 994: 43: 25$ & & & \\
\hline$-5.000525 E-05$ & $2.999311 \mathrm{E}-03$ & & & & 3/16/99 4:43:28 & & & \\
\hline$-5.000522 E-05$ & $2.999278 \mathrm{E}-03$ & & & & $3 / 16 / 994: 43: 31$ & & & \\
\hline $5.000263 \mathrm{E}-05$ & $-2.999803 E-03$ & & & & $3 / 16 / 994: 43: 34$ & & & \\
\hline $5.000266 \mathrm{E}-05$ & $-2.999823 E-03$ & & & & $3 / 16 / 994: 43: 37$ & & & \\
\hline$-5.000531 \mathrm{E}-05$ & $2.999312 \mathrm{E}-03$ & & & & $3 / 16 / 994: 43: 39$ & & & \\
\hline$-5.000530 \mathrm{E}-05$ & $2.999314 \mathrm{E}-03$ & & & & $3 / 16 / 994: 43: 42$ & & & \\
\hline $5.000266 \mathrm{E}-05$ & $-3.000096 \mathrm{E}-03$ & & & & $3 / 16 / 994: 43: 45$ & & & \\
\hline $5.000261 \mathrm{E}-05$ & $-2.999833 E-03$ & & & & $3 / 16 / 994: 43: 48$ & & & \\
\hline$-5.000536 \mathrm{E}-05$ & $2.999325 \mathrm{E}-03$ & & & & $3 / 16 / 994: 43: 51$ & & & \\
\hline$-5.000537 E-05$ & $2.999345 \mathrm{E}-03$ & & & & $3 / 16 / 994: 43: 54$ & & & \\
\hline $5.000263 \mathrm{E}-05$ & $-2.999809 E-03$ & & & & $3 / 16 / 994: 43: 57$ & & & \\
\hline $5.000262 \mathrm{E}-05$ & $-2.999811 \mathrm{E}-03$ & & & & $3 / 16 / 994: 44: 00$ & & & \\
\hline$-5.000539 E-05$ & 2.999323E-03 & & & & $3 / 16 / 994: 44: 02$ & & & \\
\hline$-5.000536 \mathrm{E}-05$ & $2.999322 \mathrm{E}-03$ & & & & $3 / 16 / 994: 44: 05$ & & & \\
\hline $5.000264 \mathrm{E}-05$ & $-2.999785 E-03$ & & & & $3 / 16 / 994: 44: 08$ & & & \\
\hline $5.000267 \mathrm{E}-05$ & $-2.999777 \mathrm{E}-03$ & & & & $3 / 16 / 994: 44: 11$ & & & \\
\hline$-5.000540 E-05$ & 2.999322E-03 & & & & $3 / 16 / 994: 44: 14$ & & & \\
\hline$-5.000543 E-05$ & 2.999339E-03 & & & & $3 / 16 / 994: 44: 17$ & & & \\
\hline $5.000266 \mathrm{E}-05$ & $-2.999807 \mathrm{E}-03$ & & & & $3 / 16 / 994: 44: 19$ & & & \\
\hline $5.000266 \mathrm{E}-05$ & $-2.999812 E-03$ & & & & $3 / 16 / 994: 44: 22$ & & & \\
\hline$-5.000538 \mathrm{E}-05$ & 2.999333E-03 & & & & $3 / 16 / 994: 44: 25$ & & & \\
\hline$-5.000541 E-05$ & $2.999358 \mathrm{E}-03$ & & & & $3 / 16 / 994: 44: 28$ & & & \\
\hline $5.000263 \mathrm{E}-05$ & $-2.999792 \mathrm{E}-03$ & & & & $3 / 16 / 994: 44: 31$ & & & \\
\hline $5.000264 \mathrm{E}-05$ & $-2.999775 \mathrm{E}-03$ & & & & $3 / 16 / 994: 44: 33$ & & & \\
\hline$-5.000542 E-05$ & $2.999339 \mathrm{E}-03$ & & & & $3 / 16 / 994: 44: 36$ & & & \\
\hline$-5.000544 E-05$ & $2.999364 \mathrm{E}-03$ & & & & $3 / 16 / 994: 44: 39$ & & & \\
\hline $5.000261 E-05$ & $-2.999780 \mathrm{E}-03$ & & & & $3 / 16 / 994: 44: 42$ & & & \\
\hline $5.000260 \mathrm{E}-05$ & $-2.999758 \mathrm{E}-03$ & & & & $3 / 16 / 994: 44: 45$ & & & \\
\hline$-5.000544 \mathrm{E}-05$ & $2.999368 \mathrm{E}-03$ & & & & $3 / 16 / 994: 44: 48$ & & & \\
\hline$-5.000539 E-05$ & $2.999346 \mathrm{E}-03$ & & & & $3 / 16 / 994: 44: 50$ & & & \\
\hline 5.000262E-05 & $-2.999765 E-03$ & & & & $3 / 16 / 994: 44: 53$ & & & \\
\hline $5.000260 \mathrm{E}-05$ & $-2.999775 \mathrm{E}-03$ & & & & $3 / 16 / 994: 44: 56$ & & & \\
\hline$-5.000539 E-05$ & 2.999367E-03 & & & & $3 / 16 / 994: 44: 59$ & & & \\
\hline$-5.000535 \mathrm{E}-05$ & $2.999348 E-03$ & & & & $3 / 16 / 994: 45: 02$ & & & \\
\hline $5.000258 \mathrm{E}-05$ & $-2.999796 \mathrm{E}-03$ & & & & $3 / 16 / 994: 45: 05$ & & & \\
\hline $5.000259 E-05$ & $-2.999775 E-03$ & & & & $3 / 16 / 994: 45: 07$ & & & \\
\hline$-5.000538 E-05$ & $2.999322 \mathrm{E}-03$ & & & & $3 / 16 / 994: 45: 10$ & & & \\
\hline$-5.000539 E-05$ & $2.999350 \mathrm{E}-03$ & & & & $3 / 16 / 994: 45: 13$ & & & \\
\hline $5.000267 \mathrm{E}-05$ & $-2.999801 E-03$ & & & & $3 / 16 / 994: 45: 16$ & & & \\
\hline $5.000400 \mathrm{E}-05$ & $2.999572 \mathrm{E}-03$ & 59.986642 & $6.468464 \mathrm{E}-04$ & 1.499909E-07 & $3 / 16 / 994: 45: 20$ & 60.51329333 & 290.8461293 & \\
\hline & & & & & & & & \\
\hline
\end{tabular}


Tungsten.xls

\begin{tabular}{|c|c|c|c|c|c|c|c|c|}
\hline Current $(A)$ & Voltage $(V)$ & Res.(ohms) & Std. Dev. Of $\Omega$ & Power $(W)$ & Date/time & Cernox (ohms) & \multicolumn{2}{|c|}{ Cernox (K) 4th order poly. } \\
\hline Sample ID $=W$ & Beam Energy $=0$ & Time on target & $t=0$ & & & & & \\
\hline \multicolumn{9}{|c|}{ Pre-irradiation resistivity data at room temperature prior to testing at $1.94 \mathrm{GeV}$} \\
\hline & & & & & & & & \\
\hline $5.000226 \mathrm{E}-04$ & $-2.999193 \mathrm{E}-02$ & & & & $3 / 16 / 994: 46: 14$ & & & \\
\hline$-5.000673 \mathrm{E}-04$ & $2.999209 \mathrm{E}-02$ & & & & $3 / 16 / 994: 46: 17$ & & & \\
\hline$-5.000671 \mathrm{E}-04$ & 2.999213E-02 & & & & $3 / 16 / 994: 46: 20$ & & & \\
\hline $5.000240 \mathrm{E}-04$ & $-2.999218 \mathrm{E}-02$ & & & & $3 / 16 / 994: 46: 23$ & & & \\
\hline $5.000242 E-04$ & $-2.999220 \mathrm{E}-02$ & & & & $3 / 16 / 994: 46: 25$ & & & \\
\hline$-5.000661 E-04$ & $2.999209 \mathrm{E}-02$ & & & & $3 / 16 / 994: 46: 28$ & & & \\
\hline$-5.000665 \mathrm{E}-04$ & $2.999214 \mathrm{E}-02$ & & & & $3 / 16 / 994: 46: 31$ & & & \\
\hline $5.000241 \mathrm{E}-04$ & $-2.999223 \mathrm{E}-02$ & & & & $3 / 16 / 994: 46: 34$ & & & \\
\hline $5.000241 \mathrm{E}-04$ & $-2.999222 \mathrm{E}-02$ & & & & $3 / 16 / 994: 46: 37$ & & & \\
\hline$-5.000671 \mathrm{E}-04$ & 2.999221E-02 & & & & $3 / 16 / 994: 46: 40$ & & & \\
\hline$-5.000674 \mathrm{E}-04$ & $2.999217 \mathrm{E}-02$ & & & & $3 / 16 / 994: 46: 42$ & & & \\
\hline $5.000241 \mathrm{E}-04$ & $-2.999223 \mathrm{E}-02$ & & & & $3 / 16 / 994: 46: 45$ & & & \\
\hline $5.000239 \mathrm{E}-04$ & $-2.999220 \mathrm{E}-02$ & & & & $3 / 16 / 994: 46: 48$ & & & \\
\hline$-5.000668 \mathrm{E}-04$ & $2.999227 \mathrm{E}-02$ & & & & $3 / 16 / 994: 46: 51$ & & & \\
\hline$-5.000668 \mathrm{E}-04$ & $2.999219 \mathrm{E}-02$ & & & & $3 / 16 / 994: 46: 54$ & & & \\
\hline $5.000242 \mathrm{E}-04$ & $-2.999224 \mathrm{E}-02$ & & & & $3 / 16 / 994: 46: 57$ & & & \\
\hline $5.000242 \mathrm{E}-04$ & $-2.999218 \mathrm{E}-02$ & & & & $3 / 16 / 994: 46: 59$ & & & \\
\hline$-5.000667 E-04$ & $2.999227 \mathrm{E}-02$ & & & & $3 / 16 / 994: 47: 02$ & & & \\
\hline$-5.000666 \mathrm{E}-04$ & $2.999220 \mathrm{E}-02$ & & & & $3 / 16 / 994: 47: 05$ & & & \\
\hline 5.000240 E-04 & $-2.999228 E-02$ & & & & $3 / 16 / 99$ 4:47:08 & & & \\
\hline $5.000239 \mathrm{E}-04$ & $-2.999221 \mathrm{E}-02$ & & & & $3 / 16 / 99$ 4:47:11 & & & \\
\hline$-5.000666 \mathrm{E}-04$ & $2.999216 \mathrm{E}-02$ & & & & $3 / 16 / 994: 47: 13$ & & & \\
\hline$-5.000669 \mathrm{E}-04$ & $2.999233 \mathrm{E}-02$ & & & & $3 / 16 / 994: 47: 16$ & & & \\
\hline $5.000239 \mathrm{E}-04$ & $-2.999227 \mathrm{E}-02$ & & & & $3 / 16 / 994: 47: 19$ & & & \\
\hline $5.000239 \mathrm{E}-04$ & $-2.999222 E-02$ & & & & $3 / 16 / 99$ 4:47:22 & & & \\
\hline$-5.000674 E-04$ & 2.999234E-02 & & & & $3 / 16 / 994: 47: 25$ & & & \\
\hline$-5.000675 \mathrm{E}-04$ & $2.999228 \mathrm{E}-02$ & & & & $3 / 16 / 994: 47: 27$ & & & \\
\hline $5.000240 \mathrm{E}-04$ & $-2.999227 \mathrm{E}-02$ & & & & $3 / 16 / 994: 47: 30$ & & & \\
\hline $5.000244 \mathrm{E}-04$ & $-2.999222 \mathrm{E}-02$ & & & & $3 / 16 / 994: 47: 33$ & & & \\
\hline$-5.000673 \mathrm{E}-04$ & $2.999229 \mathrm{E}-02$ & & & & $3 / 16 / 994: 47: 36$ & & & \\
\hline$-5.000670 \mathrm{E}-04$ & 2.999229E-02 & & & & $3 / 16 / 994: 47: 39$ & & & \\
\hline $5.000237 \mathrm{E}-04$ & $-2.999228 \mathrm{E}-02$ & & & & $3 / 16 / 994: 47: 42$ & - & - & \\
\hline $5.000237 \mathrm{E}-04$ & $-2.999224 E-02$ & & & & $3 / 16 / 994: 47: 44$ & $\cdots-$ & - & \\
\hline$-5.000670 \mathrm{E}-04$ & $2.999234 \mathrm{E}-02$ & & & & $3 / 16 / 994: 47: 47$ & & & \\
\hline$-5.000670 E-04$ & 2.999233E-02 & & & & $3 / 16 / 994: 47: 50$ & & & \\
\hline $5.000237 \mathrm{E}-04$ & $-2.999229 \mathrm{E}-02$ & & & & $3 / 16 / 994: 47: 53$ & & & \\
\hline $5.000238 E-04$ & $-2.999223 E-02$ & & & & $3 / 16 / 994: 47: 56$ & & & \\
\hline$-5.000678 \mathrm{E}-04$ & $2.999249 \mathrm{E}-02$ & & & & $3 / 16 / 994: 47: 59$ & & & \\
\hline$-5.000678 E-04$ & $2.999247 \mathrm{E}-02$ & & & & $3 / 16 / 994: 48: 01$ & & & \\
\hline $5.000235 \mathrm{E}-04$ & $-2.999226 \mathrm{E}-02$ & & & & $3 / 16 / 994: 48: 04$ & & & \\
\hline $5.000455 \mathrm{E}-04$ & 2.999224E-02 & 59.979019 & 1.408803E-04 & $1.499755 E-05$ & $3 / 16 / 99$ 4:48:08 & 60.5318003 & 290.7431149 & \\
\hline & & & & & & & & \\
\hline
\end{tabular}


Tungsten.xls

\begin{tabular}{|c|c|c|c|c|c|c|c|c|}
\hline \multirow{2}{*}{$\begin{array}{l}\text { Current }(A) \\
\text { Sample ID }=W\end{array}$} & Voltage (V) & Res.(ohms) & \multirow{2}{*}{$\begin{array}{l}\text { Std. Dev. Of } \Omega \\
\text { et }=0\end{array}$} & \multirow[t]{2}{*}{ Power (W) } & \multirow[t]{2}{*}{ Date/time } & \multirow[t]{2}{*}{ Cernox (ohms) } & \multicolumn{2}{|c|}{ Cernox $(\mathrm{K})$ 4th order poly. } \\
\hline & Beam Energy $=0$ & Time on targe & & & & & & \\
\hline \multicolumn{9}{|c|}{ Pre-irradiation resistivity data at liquid helium temperature prior to testing at $1.94 \mathrm{GeV}$} \\
\hline $5.000231 E-04$ & $-2.343763 \mathrm{E}-03$ & & & & 3/16/99 6:27:43 & & & \\
\hline$-5.000764 \mathrm{E}-04$ & $2.340531 \mathrm{E}-03$ & & & & $3 / 16 / 996: 27: 46$ & & & \\
\hline$-5.000759 \mathrm{E}-04$ & $2.340528 \mathrm{E}-03$ & & & & 3/16/99 6:27:49 & & & \\
\hline $5.000233 \mathrm{E}-04$ & $-2.343739 \mathrm{E}-03$ & & & & $3 / 16 / 996: 27: 51$ & & & \\
\hline $5.000234 \mathrm{E}-04$ & $-2.343710 \mathrm{E}-03$ & & & & $3 / 16 / 996: 27: 54$ & & & \\
\hline$-5.000759 \mathrm{E}-04$ & $2.340558 \mathrm{E}-03$ & & & & $3 / 16 / 996: 27: 57$ & & & \\
\hline$-5.000756 E-04$ & $2.340568 \mathrm{E}-03$ & & & & 3/16/99 6:28:00 & & & \\
\hline $5.000235 \mathrm{E}-04$ & $-2.343709 \mathrm{E}-03$ & & & & $3 / 16 / 996.28 .03$ & & & \\
\hline $5.000238 E-04$ & $-2.343706 \mathrm{E}-03$ & & & & 3/16/99 6:28:06 & & & \\
\hline$-5.000759 \mathrm{E}-04$ & $2.340567 \mathrm{E}-03$ & & & & $3 / 16 / 996: 28: 08$ & & & \\
\hline$-5.000760 E-04$ & $2.340570 \mathrm{E}-03$ & & & & $3 / 16 / 996: 28: 11$ & & & \\
\hline $5.000235 \mathrm{E}-04$ & $-2.343705 E-03$ & & & & $3 / 16 / 996: 28: 14$ & & & \\
\hline $5.000231 \mathrm{E}-04$ & $-2.343718 \mathrm{E}-03$ & & & & $3 / 16 / 996.28: 17$ & & & \\
\hline$-5.000757 \mathrm{E}-04$ & $2.340572 E-03$ & & & & $3 / 16 / 996: 28: 20$ & & & \\
\hline$-5.000750 \mathrm{E}-04$ & $2.340566 \mathrm{E}-03$ & & & & 3/16/99 6:28:23 & & & \\
\hline $5.000239 \mathrm{E}-04$ & $-2.343697 \mathrm{E}-03$ & & & & 3/16/99 6:28:25 & & & \\
\hline $5.000238 E-04$ & $-2.343695 \mathrm{E}-03$ & & & & $3 / 16 / 996: 28: 28$ & & & \\
\hline$-5.000759 E-04$ & $2.340580 \mathrm{E}-03$ & & & & $3 / 16 / 996: 28: 31$ & & & \\
\hline$-5.000759 \mathrm{E}-04$ & 2.340597E-03 & & & & $3 / 16 / 996: 28: 34$ & & & \\
\hline $5.000234 E-04$ & $-2.343698 \mathrm{E}-03$ & & & & $3 / 16 / 996: 28: 37$ & & & \\
\hline $5.000232 E-04$ & $-2.343708 \mathrm{E}-03$ & & & & $3 / 16 / 996: 28: 39$ & & & \\
\hline$-5.000763 \mathrm{E}-04$ & $2.340612 E-03$ & & & & $3 / 16 / 996: 28: 42$ & & & \\
\hline$-5.000765 \mathrm{E}-04$ & $2.340582 E-03$ & & & & 3/16/99 6:28:45 & & & \\
\hline 5.000224E-04 & $-2.343695 \mathrm{E}-03$ & & & & $3 / 16 / 996: 28: 48$ & & & \\
\hline $5.000226 \mathrm{E}-04$ & $-2.343701 E-03$ & & & & $3 / 16 / 996: 28: 51$ & & & \\
\hline$-5.000770 \mathrm{E}-04$ & $2.340590 \mathrm{E}-03$ & & & & $3 / 16 / 996: 28: 54$ & & & \\
\hline$-5.000760 \mathrm{E}-04$ & $2.340593 \mathrm{E}-03$ & & & & $3 / 16 / 996: 28: 56$ & & & \\
\hline $5.000234 \mathrm{E}-04$ & $-2.343685 E-03$ & & & & $3 / 16 / 996: 28: 59$ & & & \\
\hline $5.000231 E-04$ & $-2.343702 E-03$ & & & & $3 / 16 / 996: 29: 02$ & & & \\
\hline$-5.000760 \mathrm{E}-04$ & $2.340580 \mathrm{E}-03$ & & & & 3/16/99 6:29:05 & & & \\
\hline$-5.000759 \mathrm{E}-04$ & $2.340600 \mathrm{E}-03$ & & & & 3/16/99 6:29:08 & & & \\
\hline $5.000233 E-04$ & $-2.343676 \mathrm{E}-03$ & & & & $3 / 16 / 996: 29: 11$ & & & \\
\hline $5.000228 \mathrm{E}-04$ & $-2.343684 \mathrm{E}-03$ & & & & $3 / 16 / 996: 29: 13$ & & & \\
\hline$-5.000765 \mathrm{E}-04$ & $2.340616 \mathrm{E}-03$ & & & & $3 / 16 / 996: 29: 16$ & & & \\
\hline$-5.000766 \mathrm{E}-04$ & $2.340607 \mathrm{E}-03$ & & & & $3 / 16 / 996: 29: 19$ & & & \\
\hline $5.000227 \mathrm{E}-04$ & $-2.343683 E-03$ & & & & $3 / 16 / 996: 29: 22$ & & & \\
\hline $5.000235 \mathrm{E}-04$ & $-2.343703 E-03$ & & & & $3 / 16 / 996: 29: 25$ & & & \\
\hline$-5.000767 E-04$ & 2.340593E-03 & & & & $3 / 16 / 996: 29: 28$ & & & \\
\hline$-5.000765 E-04$ & 2.340590E-03 & & & & $3 / 16 / 996: 29: 30$ & & & \\
\hline $5.000227 \mathrm{E}-04$ & $-2.343663 \mathrm{E}-03$ & & & & $3 / 16 / 996: 29: 33$ & & & \\
\hline $5.000497 \mathrm{E}-04$ & $2.342141 \mathrm{E}-03$ & 4.683817 & 1.437081E-05 & 1.171179E-06 & $3 / 16 / 996: 29: 37$ & 4865.927039 & 4.786808843 & \\
\hline & & & & & & & & \\
\hline
\end{tabular}


Tungsten.xls

\begin{tabular}{|c|c|c|c|c|c|c|c|c|}
\hline Current $(A)$ & Voltage (V) & Res.(ohms) & Std. Dev. Of $\Omega$ & Power (W) & Date/time & Cernox (ohms) & Cernox $(K)$ 4th ord & er poly. \\
\hline Sample ID $=W$ & Beam Energy $=0$ & Time on targe & $t=0$ & & & & & \\
\hline Pre-irradiation resis & istivity data at liquid & helium temperature & e prior to testing at & $1.94 \mathrm{GeV}$ & & & & \\
\hline & & & & & & & & \\
\hline $9.999669 \mathrm{E}-04$ & $-4.685302 \mathrm{E}-03$ & & & & $3 / 16 / 996: 30: 24$ & & & \\
\hline$-1.000003 \mathrm{E}-03$ & $4.682180 \mathrm{E}-03$ & & & & $3 / 16 / 996: 30: 26$ & & & \\
\hline$-1.000003 E-03$ & $4.682181 \mathrm{E}-03$ & & & & $3 / 16 / 996: 30: 29$ & & & \\
\hline 9.999680 E-04 & $-4.685329 \mathrm{E}-03$ & & & & $3 / 16 / 996: 30: 32$ & & & \\
\hline $9.999675 \mathrm{E}-04$ & $-4.685323 \mathrm{E}-03$ & & & & $3 / 16 / 996: 30: 35$ & & & \\
\hline$-1.000003 E-03$ & $4.682180 \mathrm{E}-03$ & & & & $3 / 16 / 996: 30: 38$ & & & \\
\hline$-1.000003 E-03$ & 4.682197E-03 & & & & $3 / 16 / 996: 30: 41$ & & & \\
\hline $9.999665 \mathrm{E}-04$ & $-4.685290 \mathrm{E}-03$ & & & & $3 / 16 / 996: 30: 43$ & & & \\
\hline $9.999661 \mathrm{E}-04$ & $-4.685291 E-03$ & & & & $3 / 16 / 996: 30: 46$ & & & \\
\hline$-1.000003 E-03$ & $4.682212 \mathrm{E}-03$ & & & & $3 / 16 / 996: 30: 49$ & & & \\
\hline$-1.000003 E-03$ & $4.682181 \mathrm{E}-03$ & & & & $3 / 16 / 996: 30: 52$ & & & \\
\hline 9.999664E-04 & $-4.685239 E-03$ & & & & $3 / 16 / 996: 30: 55$ & & & \\
\hline 9.999663E-04 & $-4.685260 \mathrm{E}-03$ & & & & $3 / 16 / 996: 30: 58$ & & & \\
\hline$-1.000003 E-03$ & $4.682233 \mathrm{E}-03$ & & & & $3 / 16 / 996: 31: 00$ & & & \\
\hline$-1.000003 E-03$ & $4.682245 \mathrm{E}-03$ & & & & $3 / 16 / 996: 31: 03$ & & & \\
\hline $9.999664 \mathrm{E}-04$ & $-4.685250 \mathrm{E}-03$ & & & & $3 / 16 / 996: 31: 06$ & & & \\
\hline $9.999658 \mathrm{E}-04$ & $-4.685256 \mathrm{E}-03$ & & & & $3 / 16 / 996: 31: 09$ & & & \\
\hline$-1.000004 \mathrm{E}-03$ & $4.682229 \mathrm{E}-03$ & & & & $3 / 16 / 996: 31: 12$ & & & \\
\hline$-1.000003 E-03$ & $4.682269 \mathrm{E}-03$ & & & & $3 / 16 / 996: 31: 15$ & & & \\
\hline 9.999661E-04 & $-4.685251 \mathrm{E}-03$ & & & & $3 / 16 / 996: 31: 17$ & & & \\
\hline $9.999659 \mathrm{E}-04$ & $-4.685263 \mathrm{E}-03$ & & & & $3 / 16 / 996: 31: 20$ & & & \\
\hline$-1.000004 E-03$ & $4.682228 \mathrm{E}-03$ & & & & $3 / 16 / 996: 31: 23$ & & & \\
\hline$-1.000004 E-03$ & $4.682222 \mathrm{E}-03$ & & & & $3 / 16 / 996: 31: 26$ & & & \\
\hline $9.999673 \mathrm{E}-04$ & $-4.685285 \mathrm{E}-03$ & & & & $3 / 16 / 996: 31: 29$ & & & \\
\hline 9.999671E-04 & $-4.685247 \mathrm{E}-03$ & & & & $3 / 16 / 996: 31: 32$ & & & \\
\hline$-1.000003 E-03$ & $4.682212 \mathrm{E}-03$ & & & & $3 / 16 / 996: 31: 34$ & & & \\
\hline$-1.000002 E-03$ & $4.682241 \mathrm{E}-03$ & & & & $3 / 16 / 996: 31: 37$ & & & \\
\hline $9.999677 \mathrm{E}-04$ & $-4.685267 \mathrm{E}-03$ & & & & $3 / 16 / 996: 31: 40$ & & & \\
\hline $9.999666 \mathrm{E}-04$ & $-4.685278 \mathrm{E}-03$ & & & & $3 / 16 / 996: 31: 43$ & & & \\
\hline$-1.000003 E-03$ & $4.682219 \mathrm{E}-03$ & & & & $3 / 16 / 996: 31: 46$ & & & \\
\hline$-1.000003 E-03$ & $4.682278 \mathrm{E}-03$ & & & & $3 / 16 / 996: 31: 49$ & & & \\
\hline $9.999665 \mathrm{E}-04$ & $-4.685226 \mathrm{E}-03$ & & & & $3 / 16 / 996: 31: 51$ & & & \\
\hline $9.999666 \mathrm{E}-04$ & $-4.685264 \mathrm{E}-03$ & & & & $3 / 16 / 996: 31: 54$ & & & \\
\hline$-1.000004 \mathrm{E}-03$ & $4.682251 \mathrm{E}-03$ & & & & $3 / 16 / 996: 31: 57$ & & & \\
\hline$-1.000003 E-03$ & 4.682234E-03 & & & & $3 / 16 / 996: 32: 00$ & & & \\
\hline $9.999670 \mathrm{E}-04$ & $-4.685247 \mathrm{E}-03$ & & & & $3 / 16 / 996: 32: 03$ & & & \\
\hline $9.999662 \mathrm{E}-04$ & $-4.685257 \mathrm{E}-03$ & & & & $3 / 16 / 996: 32: 06$ & & & \\
\hline$-1.000003 \bar{E}-03$ & $4.682234 \mathrm{E}-03$ & & & & $3 / 16 / 996: 32: 08$ & & & \\
\hline$-1.000002 \mathrm{E}-03$ & $4.682210 \mathrm{E}-03$ & & & & $3 / 16 / 996: 32: 11$ & & & \\
\hline 9.999671E-04 & $-4.685263 \mathrm{E}-03$ & & & & $3 / 16 / 996: 32: 14$ & & & \\
\hline $9.999849 E-04$ & $4.683746 \mathrm{E}-03$ & 4.683816 & $1.059776 \mathrm{E}-05$ & $4.683665 \mathrm{E}-06$ & $3 / 16 / 996: 32: 18$ & 4866.117557 & 4.78670321 & \\
\hline & & L & & & - & - & $-\cdots$ & \\
\hline
\end{tabular}


Tungsten.xls

\begin{tabular}{|c|c|c|c|c|c|c|c|c|}
\hline \multirow{2}{*}{\begin{tabular}{|l} 
Current $(A)$ \\
Sample $I D=W$ \\
\end{tabular}} & Voltage (V) & Res.(ohms) & \multirow{2}{*}{$\frac{\text { Std. Dev. Of } \Omega}{\mathrm{et}=0}$} & \multirow[t]{2}{*}{ Power (W) } & \multirow[t]{2}{*}{ Date/time } & \multirow[t]{2}{*}{ Cernox (ohms) } & \multicolumn{2}{|c|}{ Cernox $(\mathrm{K})$ 4th order poly. } \\
\hline & Beam Energy $=0$ & Time on target & & & & & & \\
\hline \multicolumn{9}{|c|}{ Pre-irradiation resistivity data at liquid helium temperature prior to testing at $1.94 \mathrm{GeV}$} \\
\hline & & & & & & & & \\
\hline $2.000134 \mathrm{E}-03$ & $-9.370262 E-03$ & & & & 3/16/99 6:33:02 & & & \\
\hline$-2.000581 E-03$ & $9.367980 \mathrm{E}-03$ & & & & 3/16/99 6:33:05 & & & \\
\hline$-2.000581 E-03$ & $9.367941 E-03$ & & & & 3/16/99 6:33:08 & & & \\
\hline $2.000143 E-03$ & $-9.370252 \mathrm{E}-03$ & & & & $3 / 16 / 996: 33: 10$ & & & \\
\hline $2.000142 E-03$ & $-9.370309 \mathrm{E}-03$ & & & & $3 / 16 / 996: 33: 13$ & & & \\
\hline$-2.000573 E-03$ & $9.367908 \mathrm{E}-03$ & & & & 3/16/99 6:33:16 & & & \\
\hline$-2.000575 E-03$ & $9.367885 \mathrm{E}-03$ & & & & $3 / 16 / 996: 33: 19$ & & & \\
\hline $2.000150 \mathrm{E}-03$ & $-9.370258 E-03$ & & & & 3/16/99 6:33:22 & & & \\
\hline $2.000146 \mathrm{E}-03$ & $-9.370269 \mathrm{E}-03$ & & & & $3 / 16 / 996: 33: 25$ & & & \\
\hline$-2.000574 E-03$ & $9.367894 \mathrm{E}-03$ & & & & $3 / 16 / 996: 33: 27$ & & & \\
\hline$-2.000572 E-03$ & $9.367941 \mathrm{E}-03$ & & & & 3/16/99 6:33:30 & & & \\
\hline $2.000139 E-03$ & $-9.370284 E-03$ & & & & 3/16/99 6:33:33 & & & \\
\hline $2.000144 E-03$ & $-9.370340 \mathrm{E}-03$ & & & & $3 / 16 / 996: 33: 36$ & & & \\
\hline$-2.000571 \mathrm{E}-03$ & $9.367911 \mathrm{E}-03$ & & & & 3/16/99 6:33:39 & & & \\
\hline$-2.000569 E-03$ & $9.367865 \mathrm{E}-03$ & & & & $3 / 16 / 996: 33: 42$ & & & \\
\hline $2.000149 \mathrm{E}-03$ & $-9.370429 E-03$ & & & & $3 / 16 / 996: 33: 44$ & & & \\
\hline $2.000151 \mathrm{E}-03$ & $-9.370378 E-03$ & & & & $3 / 16 / 996: 33: 47$ & & & \\
\hline$-2.000573 E-03$ & $9.367883 \mathrm{E}-03$ & & & & $3 / 16 / 996: 33: 50$ & & & \\
\hline$-2.000576 \mathrm{E}-03$ & $9.367841 \mathrm{E}-03$ & & & & $3 / 16 / 996: 33: 53$ & & & \\
\hline 2.000141E-03 & $-9.370408 \mathrm{E}-03$ & & & & $3 / 16 / 996: 33: 56$ & & & \\
\hline 2.000141E-03 & $-9.370305 E-03$ & & & & $3 / 16 / 996: 33: 58$ & & & \\
\hline$-2.000577 \mathrm{E}-03$ & $9.367926 \mathrm{E}-03$ & & & & $3 / 16 / 996: 34: 01$ & & & \\
\hline$-2.000572 \mathrm{E}-03$ & $9.367963 \mathrm{E}-03$ & & & & $3 / 16 / 996: 34: 04$ & & & \\
\hline $2.000139 E-03$ & $-9.370273 E-03$ & & & & $3 / 16 / 996: 34: 07$ & & & \\
\hline $2.000140 \mathrm{E}-03$ & $-9.370303 E-03$ & & & & $3 / 16 / 996: 34: 10$ & & & \\
\hline$-2.000580 \mathrm{E}-03$ & $9.367996 \mathrm{E}-03$ & & & & $3 / 16 / 996: 34: 13$ & & & \\
\hline$-2.000575 E-03$ & $9.367969 \mathrm{E}-03$ & & & & $3 / 16 / 996: 34: 15$ & & & \\
\hline $2.000137 E-03$ & $-9.370231 E-03$ & & & & $3 / 16 / 996: 34: 18$ & & & \\
\hline $2.000138 \mathrm{E}-03$ & $-9.370221 \mathrm{E}-03$ & & & & $3 / 16 / 996: 34: 21$ & & & \\
\hline$-2.000574 E-03$ & $9.367950 \mathrm{E}-03$ & & & & $3 / 16 / 996: 34: 24$ & & & \\
\hline$-2.000571 \mathrm{E}-03$ & 9.367957E-03 & & & & $3 / 16 / 996: 34: 27$ & & & \\
\hline $2.000147 \mathrm{E}-03$ & $-9.370289 E-03$ & & & & $3 / 16 / 996: 34: 30$ & & & \\
\hline 2.000145E-03 & $-9.370368 E-03$ & & & & $3 / 16 / 996: 34: 32$ & & & \\
\hline$-2.000574 \mathrm{E}-03$ & $9.367908 \mathrm{E}-03$ & & & & $3 / 16 / 996: 34: 35$ & & & \\
\hline$-2.000578 E-03$ & $9.367935 \mathrm{E}-03$ & & & & $3 / 16 / 996: 34: 38$ & & & \\
\hline $2.000144 \mathrm{E}-03$ & $-9.370316 \mathrm{E}-03$ & & & & $3 / 16 / 996: 34: 41$ & & & \\
\hline $2.000143 E-03$ & $-9.370367 \mathrm{E}-03$ & & & & $3 / 16 / 996: 34: 44$ & & & \\
\hline$-2.000577 \mathrm{E}-03$ & $9.367915 \mathrm{E}-03$ & & & & $3 / 16 / 996: 34: 47$ & & & \\
\hline$-2.000578 \mathrm{E}-03$ & $9.367874 \mathrm{E}-03$ & & & & $3 / 16 / 996: 34: 49$ & & & \\
\hline $2.000138 \mathrm{E}-03$ & $-9.370362 \mathrm{E}-03$ & & & & $3 / 16 / 996: 34: 52$ & & & \\
\hline $2.000359 \mathrm{E}-03$ & $9.369117 \mathrm{E}-03$ & 4.683718 & 1.124679E-05 & $1.874159 \mathrm{E}-05$ & $3 / 16 / 996: 34: 56$ & 4865.633809 & 4.78697144 & \\
\hline & & & & & & & & \\
\hline & & & & & & & & \\
\hline
\end{tabular}




\begin{tabular}{|c|c|c|c|c|c|c|c|c|}
\hline Current $(A)$ & Voltage (V) & Res.(ohms) & Std. Dev. Of $\Omega$ & Power $(\mathrm{W})$ & Date/time & Cernox (ohms) & Cernox (K) 4th orde & or poly. \\
\hline Sample ID $=W$ & Beam Energy $=1.9$ & $94 \mathrm{GeV}$ & on target $=90$ minut & tes & & & & \\
\hline First data acquisitio & on after irradiation by & by $1.94 \mathrm{GeV}$ protons & for 90 minutes at 4 & $4.7 \mathrm{~K}$. & & & & \\
\hline & & & & & & & & \\
\hline $5.000228 \mathrm{E}-04$ & $-2.360925 \mathrm{E}-03$ & & & & $3 / 16 / 99$ 10:19:28 & & & \\
\hline$-5.000604 E-04$ & $2.360579 \mathrm{E}-03$ & & & & $3 / 16 / 99$ 10:19:31 & & & \\
\hline$-5.000604 \mathrm{E}-04$ & $2.360580 \mathrm{E}-03$ & & & & $3 / 16 / 99 \quad 10: 19: 34$ & & & \\
\hline $5.000239 \mathrm{E}-04$ & $-2.360928 \mathrm{E}-03$ & & & & $3 / 16 / 99$ 10:19:36 & & & \\
\hline $5.000238 E-04$ & $-2.360916 \mathrm{E}-03$ & & & & $3 / 16 / 99$ 10:19:39 & & & \\
\hline$-5.000607 E-04$ & $2.360604 \mathrm{E}-03$ & & & & $3 / 16 / 9910: 19: 42$ & & & \\
\hline$-5.000613 E-04$ & $2.360614 \mathrm{E}-03$ & & & & $3 / 16 / 99$ 10:19:45 & & & \\
\hline $5.000235 E-04$ & $-2.360912 \mathrm{E}-03$ & & & & $3 / 16 / 99 \quad 10: 19: 48$ & & & \\
\hline $5.000234 \mathrm{E}-04$ & $-2.360896 \mathrm{E}-03$ & & & & $3 / 16 / 9910: 19: 51$ & & & \\
\hline$-5.000616 \mathrm{~F}-04$ & $2.360612 \mathrm{E}-03$ & & & & $3 / 16 / 9910: 19: 53$ & & & \\
\hline$-5.000611 E-04$ & $2.360626 \mathrm{E}-03$ & & & & $3 / 16 / 99$ 10:19:56 & & & \\
\hline $5.000234 \mathrm{E}-04$ & $-2.360891 \mathrm{E}-03$ & & & & $3 / 16 / 99$ 10:19:59 & & & \\
\hline $5.000235 \mathrm{E}-04$ & $-2.360874 \mathrm{E}-03$ & & & & $3 / 16 / 99$ 10:20:02 & & & \\
\hline$-5.000611 \mathrm{E}-04$ & $2.360647 \mathrm{E}-03$ & & & & $3 / 16 / 99$ 10:20:05 & & & \\
\hline$-5.000609 \mathrm{E}-04$ & $2.360632 \mathrm{E}-03$ & & & & $3 / 16 / 9910: 20: 08$ & & & \\
\hline $5.000241 \mathrm{E}-04$ & $-2.360882 E-03$ & & & & $3 / 16 / 9910: 20: 10$ & & & \\
\hline $5.000239 E-04$ & $-2.360873 E-03$ & & & & $3 / 16 / 99$ 10:20:13 & & & \\
\hline$-5.000611 \mathrm{E}-04$ & $2.360641 \mathrm{E}-03$ & & & & $3 / 16 / 99$ 10:20:16 & & & \\
\hline$-5.000609 E-04$ & $2.360640 \mathrm{E}-03$ & & & & $3 / 16 / 99$ 10:20:19 & & & \\
\hline $5.000238 \mathrm{E}-04$ & $-2.360882 \mathrm{E}-03$ & & & & $3 / 16 / 99$ 10:20:22 & & & \\
\hline $5.000237 E-04$ & $-2.360882 \mathrm{E}-03$ & & & & $3 / 16 / 99$ 10:20:25 & & & \\
\hline$-5.000607 \mathrm{E}-04$ & $2.360635 \mathrm{E}-03$ & & & & $3 / 16 / 99$ 10:20:27 & & & \\
\hline$-5.000604 E-04$ & $2.360629 E-03$ & & & & $3 / 16 / 99$ 10:20:30 & & & \\
\hline $5.000243 E-04$ & $-2.360862 \mathrm{E}-03$ & & & & $3 / 16 / 9910: 20: 33$ & & & \\
\hline $5.000241 \mathrm{E}-04$ & $-2.360875 \mathrm{E}-03$ & & & & $3 / 16 / 99 \quad 10: 20: 36$ & & & \\
\hline$-5.000606 \mathrm{E}-04$ & $2.360625 \mathrm{E}-03$ & & & & $3 / 16 / 99$ 10:20:39 & & & \\
\hline$-5.000607 \bar{E}-04$ & $2.360621 \mathrm{E}-03$ & & & & $3 / 16 / 9910: 20: 41$ & & & \\
\hline $5.000238 \mathrm{E}-04$ & $-2.360867 E-03$ & & & & $3 / 16 / 9910: 20: 44$ & & & \\
\hline $5.000239 \mathrm{E}-04$ & $-2.360873 E-03$ & & & & $3 / 16 / 99 \quad 10: 20: 47$ & & & \\
\hline$-5.000617 \mathrm{E}-04$ & $2.360644 \mathrm{E}-03$ & & & & $3 / 16 / 99$ 10:20:50 & & & \\
\hline$-5.000611 \mathrm{E}-04$ & $2.360631 \mathrm{E}-03$ & & & & $3 / 16 / 99 \quad 10: 20: 53$ & & & \\
\hline $5.000245 E-04$ & $-2.360878 E-03$ & & & & $3 / 16 / 99$ 10:20:56 & & & \\
\hline $5.000246 \mathrm{E}-04$ & $-2.360879 \mathrm{E}-03$ & & & & $3 / 16 / 99 \quad 10: 20: 58$ & & & \\
\hline$-5.000610 E-04$ & $2.360628 \mathrm{E}-03$ & & & & $3 / 16 / 99$ 10:21:01 & & & \\
\hline$-5.000609 \mathrm{E}-04$ & $2.360638 \mathrm{E}-03$ & & & & $3 / 16 / 99$ 10:21:04 & & & \\
\hline $5.000246 \mathrm{E}-04$ & $-2.360878 \mathrm{E}-03$ & & & & $3 / 16 / 99$ 10:21:07 & & & \\
\hline $5.000243 E-04$ & $-2.360869 \mathrm{E}-03$ & & & & $3 / 16 / 99$ 10:21:10 & & & \\
\hline$-5.000611 E-04$ & $2.360648 \mathrm{E}-03$ & & & & $3 / 16 / 99$ 10:21:13 & & & \\
\hline$-5.000614 \mathrm{E}-04$ & $2.360631 \mathrm{E}-03$ & & & & $3 / 16 / 99 \quad 10: 21: 15$ & & & \\
\hline $5.000244 \mathrm{E}-04$ & $-2.360874 \mathrm{E}-03$ & & & & $3 / 16 / 9910: 21: 18$ & & & \\
\hline $5.000424 \mathrm{E}-04$ & $2.360756 \mathrm{E}-03$ & 4.721110 & $9.779165 \mathrm{E}-06$ & $1.180478 \mathrm{E}-06$ & $3 / 16 / 9910: 21: 22$ & 4882.934766 & 4.777408232 & \\
\hline & & & & & & & & \\
\hline & & & & & & & & \\
\hline
\end{tabular}


Tungsten.xls

\begin{tabular}{|c|c|c|c|c|c|c|c|c|}
\hline Current (A) & Voltage (V) & Res.(ohms) & Std. Dev. Of $\Omega$ & \multirow{2}{*}{$\frac{\text { Power }(W)}{\text { ites }}$} & \multirow{2}{*}{ Date/time } & \multirow{2}{*}{ Cernox (ohms) } & \multicolumn{2}{|c|}{ Cernox (K) 4th order poly. } \\
\hline Sample ID $=W$ & Beam Energy $=1.5$ & $.94 \mathrm{GeV}$ & on target $=90$ minut & & & & & \\
\hline \multicolumn{9}{|c|}{ First data acquisition after irradiation by $1.94 \mathrm{GeV}$ protons for 90 minutes at $4.7 \mathrm{~K}$. } \\
\hline & & & & & & & & \\
\hline $9.999716 \mathrm{E}-04$ & $-4.721160 \mathrm{E}-03$ & & & & $3 / 16 / 99 \quad 10: 22: 20$ & & & \\
\hline$-9.999922 \mathrm{E}-04$ & 4.720867E-03 & & & & $3 / 16 / 9910: 22: 22$ & & & \\
\hline$-9.999919 \mathrm{E}-04$ & $4.720867 \mathrm{E}-03$ & & & & $3 / 16 / 9910: 22: 25$ & & & \\
\hline $9.999728 \mathrm{E}-04$ & $-4.721169 \mathrm{E}-03$ & & & & $3 / 16 / 9910: 22: 28$ & & & \\
\hline $9.999719 \mathrm{E}-04$ & $-4.721161 \mathrm{E}-03$ & & & & $3 / 16 / 99$ 10:22:31 & & & \\
\hline$-9.999926 \mathrm{E}-04$ & $4.720887 \mathrm{E}-03$ & & & & $3 / 16 / 99$ 10:22:34 & & & \\
\hline$-9.999924 \mathrm{E}-04$ & $4.720885 E-03$ & & & & $3 / 16 / 9910: 22: 36$ & & & \\
\hline 9.999723E-04 & $-4.721152 \mathrm{E}-03$ & & & & $3 / 16 / 99 \quad 10: 22: 39$ & & & \\
\hline $9.999725 E-04$ & $-4.721152 \mathrm{E}-03$ & & & & $3 / 16 / 99$ 10:22:42 & & & \\
\hline$-9.999935 \mathrm{~F}-04$ & $4720894 \mathrm{E}-03$ & & & & $3 / 16 / 9910: 22: 45$ & & & \\
\hline$-9.999931 E-04$ & $4.720899 \mathrm{E}-03$ & & & & $3 / 16 / 9910: 22: 48$ & & & \\
\hline $9.999722 \mathrm{E}-04$ & $-4.721136 \mathrm{E}-03$ & & & & $3 / 16 / 99$ 10:22:51 & & & \\
\hline $9.999716 \mathrm{E}-04$ & $-4.721146 \mathrm{E}-03$ & & & & $3 / 16 / 99 \quad 10: 22: 53$ & & & \\
\hline$-9.999936 \mathrm{E}-04$ & $4.720915 \mathrm{E}-03$ & & & & $3 / 16 / 99$ 10:22:56 & & & \\
\hline$-9.999938 \mathrm{E}-04$ & $4.720918 \mathrm{E}-03$ & & & & $3 / 16 / 99$ 10:22:59 & & & \\
\hline $9.999730 \mathrm{E}-04$ & $-4.721131 \mathrm{E}-03$ & & & & $3 / 16 / 99 \quad 10: 23: 02$ & & & \\
\hline $9.999722 \mathrm{E}-04$ & $-4.721134 \mathrm{E}-03$ & & & & $3 / 16 / 99$ 10:23:05 & & & \\
\hline$-9.999943 \mathrm{E}-04$ & $4.720913 \mathrm{E}-03$ & & & & $3 / 16 / 99$ 10:23:08 & & & \\
\hline$-9.999939 \mathrm{E}-04$ & $4.720926 \mathrm{E}-03$ & & & & $3 / 16 / 99$ 10:23:10 & & & \\
\hline 9.999726E-04 & $-4.721128 E-03$ & & & & $3 / 16 / 99 \quad 10: 23: 13$ & & & \\
\hline $9.999719 \mathrm{E}-04$ & $-4.721111 \mathrm{E}-03$ & & & & $3 / 16 / 99 \quad 10: 23: 16$ & & & \\
\hline$-9.999942 E-04$ & 4.720909E-03 & & & & $3 / 16 / 99$ 10:23:19 & & & \\
\hline$-9.999940 \mathrm{E}-04$ & 4.720923E-03 & & & & $3 / 16 / 99$ 10:23:22 & & & \\
\hline $9.999720 \mathrm{E}-04$ & $-4.721113 E-03$ & & & & $3 / 16 / 99 \quad 10: 23: 25$ & & & \\
\hline $9.999716 E-04$ & $-4.721124 \mathrm{E}-03$ & & & & 3/16/99 10:23:27 & & & \\
\hline$-9.999940 \mathrm{E}-04$ & $4.720914 \mathrm{E}-03$ & & & & $3 / 16 / 99 \quad 10: 23: 30$ & & & \\
\hline$-9.999940 \mathrm{E}-04$ & 4.720922E-03 & & & & $3 / 16 / 99 \quad 10: 23: 33$ & & & \\
\hline $9.999721 \mathrm{E}-04$ & $-4.721123 E-03$ & & & & $3 / 16 / 9910: 23: 36$ & & & \\
\hline $9.999720 \mathrm{E}-04$ & $-4.721121 \mathrm{E}-03$ & & & & $3 / 16 / 99 \quad 10: 23: 39$ & & & \\
\hline$-9.999938 \mathrm{E}-04$ & $4.720919 E-03$ & & & & $3 / 16 / 99 \quad 10: 23: 42$ & & & \\
\hline$-9.999936 \mathrm{E}-04$ & 4.720930E-03 & & & & $3 / 16 / 9910: 23: 44$ & & & \\
\hline $9.999721 \mathrm{E}-04$ & $-4.721111 \mathrm{E}-03$ & & & & $3 / 16 / 99 \quad 10: 23: 47$ & & & \\
\hline $9.999715 \mathrm{E}-04$ & $-4.721110 \mathrm{E}-03$ & & & & $3 / 16 / 99 \quad 10: 23: 50$ & & & \\
\hline$-9.999936 \mathrm{E}-04$ & $4.720925 \mathrm{E}-03$ & & & & $3 / 16 / 9910: 23: 53$ & & & \\
\hline$-9.999932 E-04$ & 4.720911E-03 & & & & $3 / 16 / 99 \quad 10: 23: 56$ & & & \\
\hline $9.999716 \mathrm{E}-04$ & $-4.721106 \mathrm{E}-03$ & & & & $3 / 16 / 99 \quad 10: 23: 58$ & & & \\
\hline $9.999715 \mathrm{E}-04$ & $-4.721108 \mathrm{E}-03$ & & & & $3 / 16 / 9910: 24: 01$ & & & \\
\hline$-9.999936 \mathrm{E}-04$ & 4.720923E-03 & & & & $3 / 16 / 99 \quad 10: 24: 04$ & & & \\
\hline$-9.999935 \mathrm{E}-04$ & 4.720912E-03 & & & & $3 / 16 / 99$ 10:24:07 & & & \\
\hline $9.999721 \mathrm{E}-04$ & $-4.721116 \mathrm{E}-03$ & & & & $3 / 16 / 99 \quad 10: 24: 10$ & & & \\
\hline $9.999828 \mathrm{E}-04$ & $4.721019 \mathrm{E}-03$ & 4.721101 & $4.959436 \mathrm{E}-06$ & 4.720933E-06 & $3 / 16 / 99$ 10:24:14 & 4884.065312 & 4.776785446 & \\
\hline & & & & & & & & \\
\hline & & & & & & & & \\
\hline
\end{tabular}


Tungsten.xls

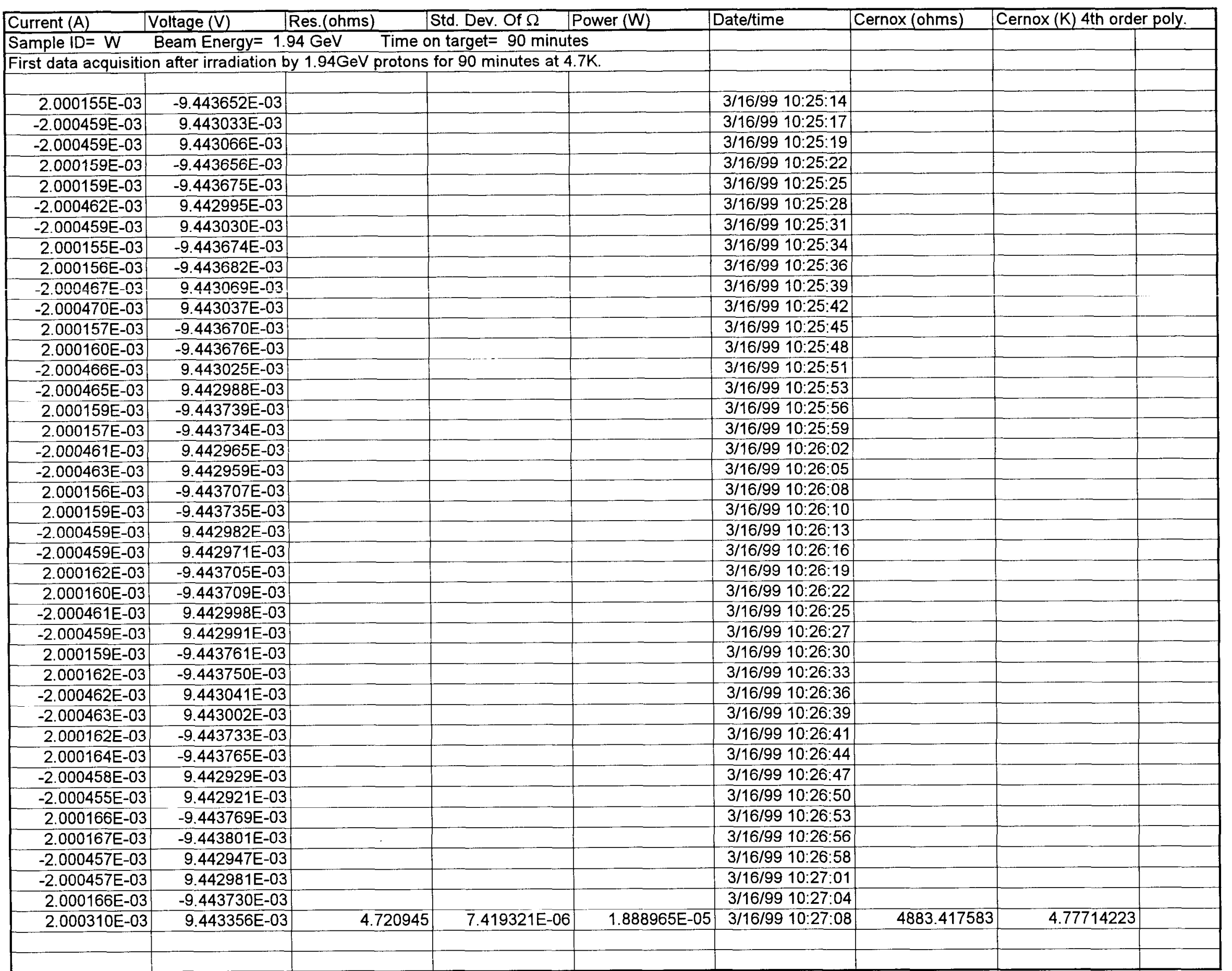


Tungsten. $x / 5$

\begin{tabular}{|c|c|c|c|c|c|c|c|c|}
\hline Current (A) & Voltage (V) & Res.(ohms) & Std. Dev. Of $\Omega$ & Power $(W)$ & Date/time & Cernox (ohms) & Cernox $(K)$ 4th ord & er poly. \\
\hline Sample ID $=\mathrm{W}$ & Beam Energy $=1.9$ & $94 \mathrm{GeV}$ & on target $=2.5$ hour & & & & & \\
\hline Second cold data a & acquisition at $1.94 \mathrm{G}$ & GeV after 2.5 hours & irradiation & & & & & \\
\hline & & & & & & & & \\
\hline $5.000235 \mathrm{E}-04$ & $-2.374452 \mathrm{E}-03$ & & & & $3 / 16 / 99$ 13:08:35 & & & \\
\hline$-5.000640 E-04$ & $2.373314 \mathrm{E}-03$ & & & & $3 / 16 / 99$ 13:08:38 & & & \\
\hline$-5.000639 E-04$ & $2.373323 E-03$ & & & & 3/16/99 13:08:40 & & & \\
\hline $5.000237 \mathrm{E}-04$ & $-2.374445 E-03$ & & & & $3 / 16 / 99$ 13:08:43 & & & \\
\hline $5.000239 E-04$ & $-2.374449 \mathrm{E}-03$ & & & & $3 / 16 / 99$ 13:08:46 & & & \\
\hline$-5.000642 E-04$ & 2.373351E-03 & & & & $3 / 16 / 99$ 13:08:49 & & & \\
\hline$-5.000642 E-04$ & $2.373342 \mathrm{E}-03$ & & & & $3 / 16 / 99$ 13:08:52 & & & \\
\hline $5.000238 \mathrm{E}-04$ & $-2.374441 \mathrm{E}-03$ & & & & $3 / 16 / 99$ 13:08:55 & & & \\
\hline $5.000238 E-04$ & $-2.374443 E-03$ & & & & 3/16/99 13:08:57 & & & \\
\hline-5.000844 드-04 & 2.373336E-03 & & & & 3/16/99 13:09:00 & & & \\
\hline$-5.000644 \mathrm{E}-04$ & $2.373321 \mathrm{E}-03$ & & & & 3/16/99 13:09:03 & & & \\
\hline $5.000239 E-04$ & $-2.374449 \mathrm{E}-03$ & & & & 3/16/99 13:09:06 & & & \\
\hline $5.000238 E-04$ & $-2.374478 E-03$ & & & & 3/16/99 13:09:09 & & & \\
\hline$-5.000649 \mathrm{E}-04$ & 2.373295E-03 & & & & $3 / 16 / 99$ 13:09:12 & & & \\
\hline$-5.000647 \mathrm{E}-04$ & $2.373294 \mathrm{E}-03$ & & & & 3/16/99 13:09:14 & & & \\
\hline $5.000239 \mathrm{E}-04$ & $-2.374492 \mathrm{E}-03$ & & & & 3/16/99 13:09:17 & & & \\
\hline $5.000240 \mathrm{E}-04$ & $-2.374512 \mathrm{E}-03$ & & & & $3 / 16 / 99$ 13:09:20 & & & \\
\hline$-5.000640 E-04$ & $2.373266 \mathrm{E}-03$ & & & & $3 / 16 / 99$ 13:09:23 & & & \\
\hline$-5.000638 \mathrm{E}-04$ & $2.373265 \mathrm{E}-03$ & & & & 3/16/99 13:09:26 & & & \\
\hline $5.000242 \mathrm{E}-04$ & $-2.374503 E-03$ & & & & 3/16/99 13:09:29 & & & \\
\hline $5.000241 E-04$ & $-2.374515 E-03$ & & & & 3/16/99 13:09:31 & & & \\
\hline$-5.000641 \mathrm{E}-04$ & $2.373252 \mathrm{E}-03$ & & & & $3 / 16 / 99$ 13:09:34 & & & \\
\hline$-5.000641 \mathrm{E}-04$ & $2.373260 \mathrm{E}-03$ & & & & $3 / 16 / 99$ 13:09:37 & & & \\
\hline $5.000232 E-04$ & $-2.374517 \mathrm{E}-03$ & & & & $3 / 16 / 99$ 13:09:40 & & & \\
\hline $5.000235 \mathrm{E}-04$ & $-2.374499 \mathrm{E}-03$ & & & & $3 / 16 / 99 \quad 13: 09: 43$ & & & \\
\hline$-5.000642 \mathrm{E}-04$ & $2.373276 \mathrm{E}-03$ & & & & $3 / 16 / 99$ 13:09:45 & & & \\
\hline$-5.000642 E-04$ & $2.373269 \mathrm{E}-03$ & & & & $3 / 16 / 99$ 13:09:48 & & & \\
\hline $5.000236 \mathrm{E}-04$ & $-2.374483 \mathrm{E}-03$ & & & & 3/16/99 13:09:51 & & & \\
\hline $5.000236 \mathrm{E}-04$ & $-2.374481 E-03$ & & & & 3/16/99 13:09:54 & & & \\
\hline$-5.000643 \mathrm{E}-04$ & $2.373290 \mathrm{E}-03$ & & & & $3 / 16 / 99$ 13:09:57 & & & \\
\hline$-5.000645 E-04$ & $2.373298 \mathrm{E}-03$ & & & & 3/16/99 13:10:00 & & & \\
\hline $5.000232 E-04$ & $-2.374458 E-03$ & & & & $3 / 16 / 9913: 10: 02$ & & & \\
\hline $5.000231 \mathrm{E}-04$ & $-2.374453 E-03$ & & & & 3/16/99 13:10:05 & & & \\
\hline$-5.000645 \mathrm{E}-04$ & $2.373308 \mathrm{E}-03$ & & & & 3/16/99 13:10:08 & & & \\
\hline$-5.000641 E-04$ & $2.373309 \mathrm{E}-03$ & & & & $3 / 16 / 99$ 13:10:11 & & & \\
\hline 5.000237E-04 & $-2.374454 \mathrm{E}-03$ & & & & $3 / 16 / 99$ 13:10:14 & & & \\
\hline $5.000231 \mathrm{E}-04$ & $-2.374450 \mathrm{E}-03$ & & & & 3/16/99 13:10:17 & & & \\
\hline$-5.000646 E-04$ & $2.373322 \mathrm{E}-03$ & & & & 3/16/99 13:10:19 & & & \\
\hline$-5.000641 \mathrm{E}-04$ & $2.373324 \mathrm{E}-03$ & & & & $3 / 16 / 99$ 13:10:22 & & & \\
\hline $5.000234 \mathrm{E}-04$ & $-2.374434 E-03$ & & & & $3 / 16 / 99$ 13:10:25 & & & \\
\hline $5.000440 E-04$ & $2.373886 \mathrm{E}-03$ & 4.747354 & $1.029432 \mathrm{E}-05$ & $1.187043 \mathrm{E}-06$ & $3 / 16 / 99$ 13:10:29 & 4860.524309 & 4.789807508 & \\
\hline & & & & & & & & \\
\hline & & & & & & & & \\
\hline
\end{tabular}


Tungsten.xls

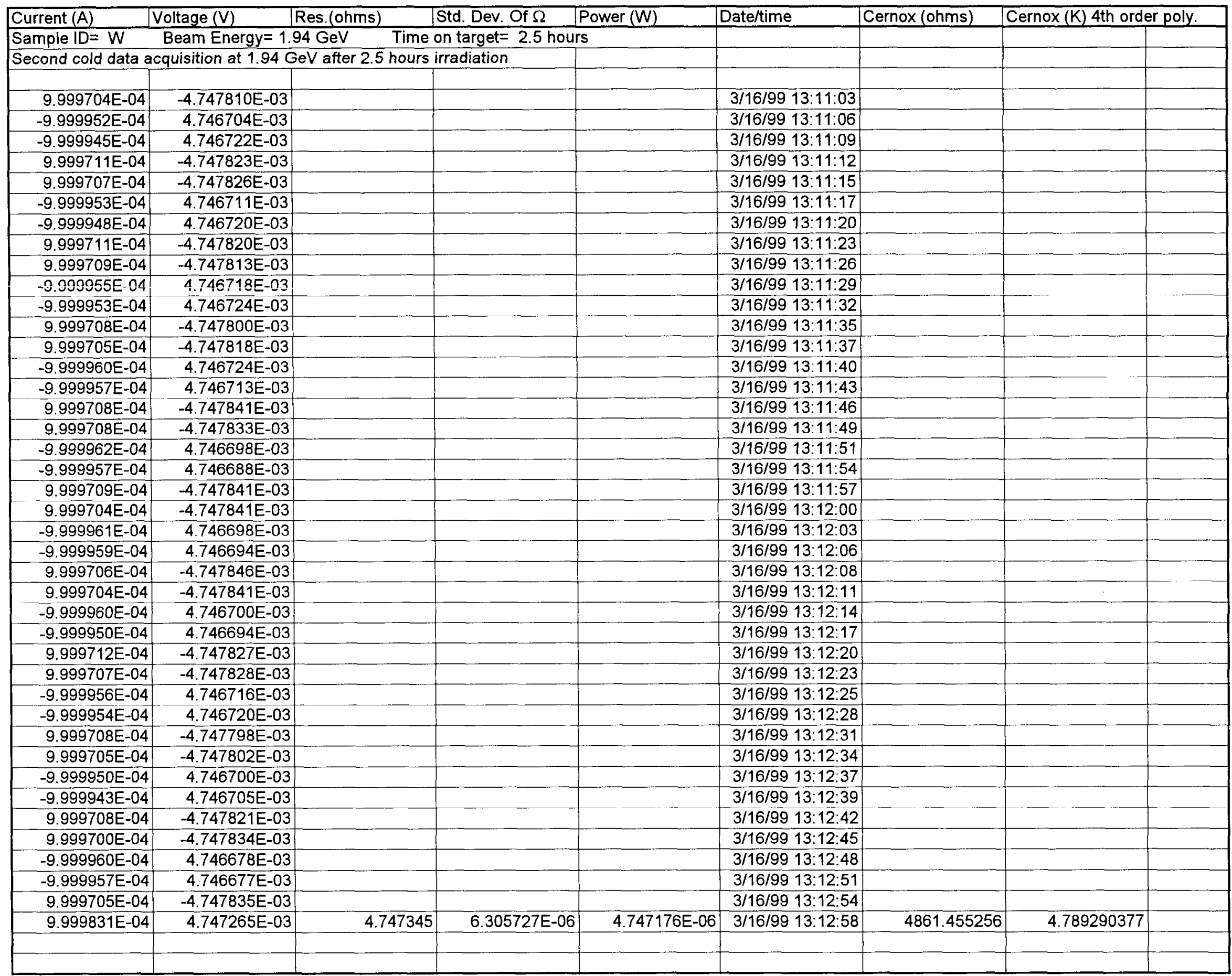


Tungsten.xls

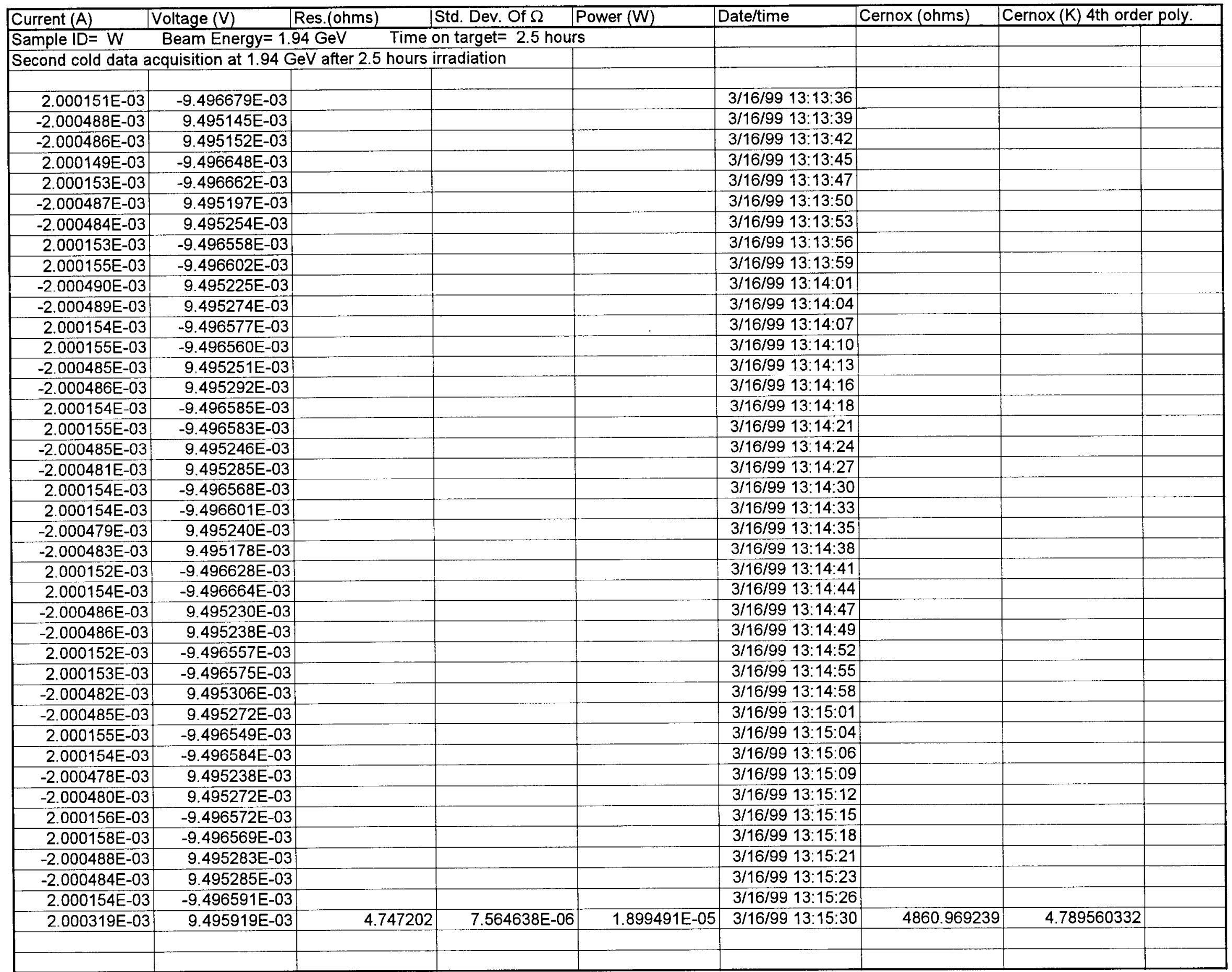




\begin{tabular}{|c|c|c|c|c|c|c|c|c|}
\hline Current (A) & Voltage (V) & Res.(ohms) & Std. Dev. Of $\Omega$ & Power (W) & Date/time & Cernox (ohms) & Cernox (K) 4th ord & er poly. \\
\hline Sample ID $=W$ & Beam Energy $=1.9$ & $.94 \mathrm{GeV}$ & on target $=$ approxi & mately 13 minutes & & & & \\
\hline Run aborted after 1 & 13 minutes at $1.94 \mathrm{G}$ & $\mathrm{GeV}$ due to upset $\mathrm{co}$ & onditions in LHe sup & pply flow rate & & & & \\
\hline & & & & & & & & \\
\hline $5.000226 \mathrm{E}-04$ & $-2.378296 \mathrm{E}-03$ & & & & $3 / 16 / 9914: 58: 08$ & & & \\
\hline$-5.000594 \mathrm{E}-04$ & $2.376555 \mathrm{E}-03$ & & & & $3 / 16 / 99$ 14:58:10 & & & \\
\hline$-5.000598 \mathrm{E}-04$ & $2.376561 \mathrm{E}-03$ & & & & $3 / 16 / 9914: 58: 13$ & & & \\
\hline $5.000228 E-04$ & $3.503903 E+01$ & & & & $3 / 16 / 99$ 14:58:16 & & & \\
\hline $5.000231 \mathrm{E}-04$ & $-2.378462 E-03$ & & & & $3 / 16 / 9914: 58: 19$ & & & \\
\hline$-5.000602 \mathrm{E}-04$ & $2.376525 E-03$ & & & & $3 / 16 / 9914: 58: 21$ & & & \\
\hline$-5.000600 \mathrm{E}-04$ & $2.376553 \mathrm{E}-03$ & & & & $3 / 16 / 9914: 58: 24$ & & & \\
\hline $5.000231 \mathrm{E}-04$ & $-2.378347 \mathrm{E}-03$ & & & & $3 / 16 / 9914: 58: 27$ & & & \\
\hline $5.000235 \mathrm{E}-04$ & $-2.378318 E-03$ & & & & $3 / 16 / 99$ 14:58:30 & & & \\
\hline$-5.000599 \mathrm{E}-04$ & $2.376549 \mathrm{E}-03$ & & & & $3 / 16 / 9914: 58: 33$ & & & \\
\hline$-5.000595 \mathrm{E}-04$ & $2.376550 \mathrm{E}-03$ & & & & $3 / 16 / 99$ 14:58:35 & & & \\
\hline $5.000232 \mathrm{E}-04$ & $-2.378323 E-03$ & & & & $3 / 16 / 9914: 58: 38$ & & & \\
\hline $5.000234 E-04$ & $-2.378336 \mathrm{E}-\mathrm{U3}$ & & & & $3 / 16 / 9914: 58: 41$ & & & \\
\hline$-5.000601 \mathrm{E}-04$ & $2.376559 \mathrm{E}-03$ & & & & $3 / 16 / 9914: 58: 44$ & & & \\
\hline$-5.000599 \mathrm{E}-04$ & $2.376562 \mathrm{E}-03$ & & & & $3 / 16 / 9914: 58: 47$ & & & \\
\hline $5.000234 \mathrm{E}-04$ & $-2.378313 \mathrm{E}-03$ & & & & $3 / 16 / 99$ 14:58:50 & & & \\
\hline 5.000232E-04 & $-2.378310 \mathrm{E}-03$ & & & & $3 / 16 / 99$ 14:58:52 & & & \\
\hline$-5.000602 E-04$ & $2.376576 \mathrm{E}-03$ & & & & $3 / 16 / 99$ 14:58:55 & & & \\
\hline$-5.000605 \mathrm{E}-04$ & $2.376574 \mathrm{E}-03$ & & & & $3 / 16 / 99$ 14:58:58 & & & \\
\hline 5.000231E-04 & $-2.378323 E-03$ & & & & $3 / 16 / 99$ 14:59:01 & & & \\
\hline $5.000230 \mathrm{E}-04$ & $-2.378319 \mathrm{E}-03$ & & & & 3/16/99 14:59:04 & & & \\
\hline$-5.000604 \mathrm{E}-04$ & $2.376589 \mathrm{E}-03$ & & & & 3/16/99 14:59:07 & & & \\
\hline$-5.000603 \mathrm{E}-04$ & 2.376581E-03 & & & & $3 / 16 / 99$ 14:59:09 & & & \\
\hline $5.000231 \mathrm{E}-04$ & $-2.378304 E-03$ & & & & $3 / 16 / 9914: 59: 12$ & & & \\
\hline $5.000227 \mathrm{E}-04$ & $-2.378307 \mathrm{E}-03$ & & & & $3 / 16 / 9914: 59: 15$ & & & \\
\hline$-5.000603 E-04$ & $2.376578 \mathrm{E}-03$ & & & & $3 / 16 / 9914: 59: 18$ & & & \\
\hline$-5.000606 E-04$ & $2.376583 \mathrm{E}-03$ & & & & $3 / 16 / 9914: 59: 21$ & & & \\
\hline $5.000229 \mathrm{E}-04$ & $-2.378290 \mathrm{E}-03$ & & & & $3 / 16 / 99$ 14:59:24 & & & \\
\hline 5.000227E-04 & $-2.378300 \mathrm{E}-03$ & & & & $3 / 16 / 9914: 59: 26$ & & & \\
\hline$-5.000614 E-04$ & $2.376589 \mathrm{E}-03$ & & & & $3 / 16 / 9914: 59: 29$ & & & \\
\hline$-5.000611 \mathrm{E}-04$ & $2.376587 \mathrm{E}-03$ & & & & $3 / 16 / 9914: 59: 32$ & & & \\
\hline $5.000228 \mathrm{E}-04$ & $-2.378308 \mathrm{E}-03$ & & & & $3 / 16 / 9914: 59: 35$ & & & \\
\hline $5.000235 \mathrm{E}-04$ & $-2.378312 E-03$ & & & & $3 / 16 / 9914: 59: 38$ & & & \\
\hline$-5.000613 \mathrm{E}-04$ & $2.376581 \mathrm{E}-03$ & & & & $3 / 16 / 9914: 59: 40$ & & & \\
\hline$-5.000613 \mathrm{E}-04$ & $2.376576 \mathrm{E}-03$ & & & & $3 / 16 / 9914: 59: 43$ & & & \\
\hline $5.000239 \mathrm{E}-04$ & $-2.378316 \mathrm{E}-03$ & & & & $3 / 16 / 99$ 14:59:46 & & & \\
\hline $5.000239 \mathrm{E}-04$ & $-2.378309 \mathrm{E}-03$ & & & & $3 / 16 / 9914: 59: 49$ & & & \\
\hline$-5.000612 \mathrm{E}-04$ & $2.376586 \mathrm{E}-03$ & & & & $3 / 16 / 9914: 59: 52$ & & & \\
\hline$-5.000610 \mathrm{E}-04$ & $2.376583 \mathrm{E}-03$ & & & & $3 / 16 / 9914: 59: 55$ & & & \\
\hline 5.000233E-04 & $-2.378311 \mathrm{E}-03$ & & & & $3 / 16 / 99$ 14:59:57 & & & \\
\hline $5.000418 \mathrm{E}-04$ & 8.782937E-01 & 1756.442191 & $7.635430 \mathrm{E}+03$ & $1.188824 \mathrm{E}-06$ & $3 / 16 / 99$ 15:00:02 & 2416.030697 & 7.379418476 & \\
\hline & & & & & & & & \\
\hline & & & & & & & & \\
\hline
\end{tabular}


Tungsten.xls

\begin{tabular}{|c|c|c|c|c|c|c|c|c|}
\hline \multirow{2}{*}{\begin{tabular}{|l} 
Current $(A)$ \\
Sample ID=W
\end{tabular}} & Voltage (V) & Res.(ohms) & Std. Dev. Of $\Omega$ & Power (W) & \multirow[t]{2}{*}{ Date/time } & \multirow[t]{2}{*}{ Cernox (ohms) } & \multicolumn{2}{|c|}{ Cernox $(\mathrm{K})$ 4th order poly. } \\
\hline & Beam Energy $=1.9$ & $94 \mathrm{GeV}$ & on target $=$ approxin & mately 13 minutes & & & & \\
\hline \multicolumn{9}{|c|}{ Run aborted after 13 minutes at $1.94 \mathrm{GeV}$ due to upset conditions in LHe supply flow rate } \\
\hline & & & & & & & & \\
\hline 9.999715E-04 & $-4.755299 E-03$ & & & & $3 / 16 / 99$ 15:00:29 & & & \\
\hline$-9.999925 E-04$ & $4.753565 \mathrm{E}-03$ & & & & $3 / 16 / 99$ 15:00:32 & & & \\
\hline$-9.999924 E-04$ & $4.753566 \mathrm{E}-03$ & & & & $3 / 16 / 9915: 00: 34$ & & & \\
\hline $9.999716 \mathrm{E}-04$ & $-4.755307 \mathrm{E}-03$ & & & & $3 / 16 / 99$ 15:00:37 & & & \\
\hline $9.999711 E-04$ & $-4.755288 E-03$ & & & & $3 / 16 / 99$ 15:00:40 & & & \\
\hline$-9.999929 E-04$ & 4.753567E-03 & & & & $3 / 16 / 99$ 15:00:43 & & & \\
\hline$-9.999928 E-04$ & 4.753554E-03 & & & & $3 / 16 / 99$ 15:00:46 & & & \\
\hline $9.999714 \mathrm{E}-04$ & $-4.755320 \mathrm{E}-03$ & & & & $3 / 16 / 99$ 15:00:48 & & & \\
\hline $9.999708 \mathrm{E}-04$ & $-4.755317 E-03$ & & & & $3 / 16 / 99$ 15:00:51 & & & \\
\hline$-9.999936 \mathrm{E}-04$ & $4.753554 \mathrm{E}-03$ & & & & $3 / 16 / 99$ 15:00:54 & & & \\
\hline$-9.999931 E-04$ & 4.753565E-03 & & & & $3 / 16 / 9915: 00: 57$ & & & \\
\hline $9.999715 \mathrm{E}-04$ & $-4.755323 E-03$ & & & & 3/16/99 15:01:00 & & & \\
\hline $9.999715 \mathrm{E}-04$ & $-4.755337 \mathrm{E}-03$ & & & & $3 / 16 / 99$ 15:01:03 & & & \\
\hline$-9.999941 \mathrm{E}-04$ & $4.753563 \mathrm{E}-03$ & & & & $3 / 16 / 99$ 15:01:05 & & & \\
\hline$-9.999932 \mathrm{E}-04$ & $4.753545 E-03$ & & & & $3 / 16 / 9915: 01: \overline{08}$ & & & \\
\hline $9.999721 \mathrm{E}-04$ & $-4.755338 \mathrm{E}-03$ & & & & $3 / 16 / 9915: 01: 11$ & & & \\
\hline $9.999713 \mathrm{E}-04$ & $-4.755323 E-03$ & & & & $3 / 16 / 99$ 15:01:14 & & & \\
\hline$-9.999939 E-04$ & $4.753558 \mathrm{E}-03$ & & & & $3 / 16 / 99$ 15:01:17 & & & \\
\hline$-9.999936 \mathrm{E}-04$ & $4.753560 \mathrm{E}-03$ & & & & $3 / 16 / 99$ 15:01:20 & & & \\
\hline $9.999714 \mathrm{E}-04$ & $-4.755327 E-03$ & & & & $3 / 16 / 99$ 15:01:22 & & & \\
\hline 9.999707E-04 & $-4.755352 E-03$ & & & & $3 / 16 / 99$ 15:01:25 & & & \\
\hline$-9.999939 E-04$ & 4.753551E-03 & & & & $3 / 16 / 99$ 15:01:28 & & & \\
\hline$-9.999939 E-04$ & 4.753579E-03 & & & & $3 / 16 / 99$ 15:01:31 & & & \\
\hline $9.999708 E-04$ & $-4.755349 \mathrm{E}-03$ & & & & $3 / 16 / 99$ 15:01:34 & & & \\
\hline $9.999709 \mathrm{E}-04$ & $-4.755347 \mathrm{E}-03$ & & & & $3 / 16 / 9915: 01: 36$ & & & \\
\hline$-9.999935 E-04$ & $4.753541 \mathrm{E}-03$ & & & & $3 / 16 / 9915: 01: 39$ & & & \\
\hline$-9.999929 E-04$ & $4.753570 \mathrm{E}-03$ & & & & $3 / 16 / 9915: 01: 42$ & & & \\
\hline $9.999713 \mathrm{E}-04$ & $-4.755339 \mathrm{E}-03$ & & & & $3 / 16 / 9915: 01: 45$ & & & \\
\hline $9.999708 \mathrm{E}-04$ & $-4.755356 \mathrm{E}-03$ & & & & $3 / 16 / 9915: 01: 48$ & & & \\
\hline$-9.999933 E-04$ & 4.753548E-03 & & & & $3 / 16 / 99$ 15:01:51 & & & \\
\hline$-9.999932 \mathrm{E}-04$ & $4.753554 \mathrm{E}-03$ & & & & $3 / 16 / 9915: 01: 53$ & & & \\
\hline 9.999719E-04 & $-4.755361 \mathrm{E}-03$ & & & & $3 / 16 / 9915: 01: 56$ & & & \\
\hline $9.999715 \mathrm{E}-04$ & $-4.755360 \mathrm{E}-03$ & & & & $3 / 16 / 99$ 15:01:59 & & & \\
\hline$-9.999935 \mathrm{E}-04$ & $4.753551 \mathrm{E}-03$ & & & & $3 / 16 / 9915: 02: 02$ & & & \\
\hline$-9.999933 E-04$ & $4.753532 \mathrm{E}-03$ & & & & $3 / 16 / 99$ 15:02:05 & & & \\
\hline $9.999718 \mathrm{E}-04$ & $-4.755368 E-03$ & & & & $3 / 16 / 9915: 02: 08$ & & & \\
\hline $9.999708 \mathrm{E}-04$ & $-4.755363 \mathrm{E}-03$ & & & & $3 / 16 / 99$ 15:02:10 & & & \\
\hline$-9.999936 \mathrm{E}-04$ & $4.753544 E-03$ & & & & $3 / 16 / 99$ 15:02:13 & & & \\
\hline$-9.999933 E-04$ & $4.753538 \mathrm{E}-03$ & & & & $3 / 16 / 99$ 15:02:16 & & & \\
\hline $9.999712 \mathrm{E}-04$ & $-4.755367 E-03$ & & & & $3 / 16 / 9915: 02: 19$ & & & \\
\hline $9.999823 \mathrm{E}-04$ & 4.754446E-03 & 4.754530 & $8.856021 E-06$ & $4.754368 \mathrm{E}-06$ & $3 / 16 / 99$ 15:02:23 & 2391.578925 & 7.430594946 & \\
\hline & & & & & & & & \\
\hline & & & & & & & & \\
\hline
\end{tabular}




\begin{tabular}{|c|c|c|c|c|c|c|c|c|}
\hline Current $(A)$ & Voltage (V) & Res.(ohms) & Std. Dev. Of $\Omega$ & Power (W) & Date/time & Cernox (ohms) & Cernox (K) 4th orde & er poly. \\
\hline Sample ID $=W$ & Beam Energy $=1$. & $.94 \mathrm{GeV}$ & on target $=$ approxin & mately 13 minutes & & & & \\
\hline Run aborted after 1 & 13 minutes at $1.94 \mathrm{C}$ & GeV due to upset co & onditions in LHe sup & pply flow rate & & & & \\
\hline & & & & & & & & \\
\hline $2.000152 \mathrm{E}-03$ & $-9.511435 \mathrm{E}-03$ & & & & $3 / 16 / 99$ 15:02:53 & & & \\
\hline$-2.000456 E-03$ & $9.509066 \mathrm{E}-03$ & & & & $3 / 16 / 99$ 15:02:56 & & & \\
\hline$-2.000453 E-03$ & $9.509047 \mathrm{E}-03$ & & & & $3 / 16 / 99$ 15:02:59 & & & \\
\hline $2.000159 \mathrm{E}-03$ & $-9.511450 \mathrm{E}-03$ & & & & $3 / 16 / 99$ 15:03:02 & & & \\
\hline $2.000159 E-03$ & $-9.511442 E-03$ & & & & $3 / 16 / 99$ 15:03:04 & & & \\
\hline$-2.000457 E-03$ & $9.509078 \mathrm{E}-03$ & & & & 3/16/99 15:03:07 & & & \\
\hline$-2.000457 E-03$ & $9.509082 \mathrm{E}-03$ & & & & $3 / 16 / 9915: 03: 10$ & & & \\
\hline $2.000156 \mathrm{E}-03$ & $-9.511463 E-03$ & & & & $3 / 16 / 99$ 15:03:13 & & & \\
\hline $2.000157 \mathrm{E}-03$ & $-9.511446 \mathrm{E}-03$ & & & & $3 / 16 / 99$ 15:03:16 & & & \\
\hline$-2.000458 \mathrm{E}-03$ & $9.509046 \mathrm{E}-03$ & & & & $3 / 16 / 99$ 15:03:19 & & & \\
\hline$-2.000457 \mathrm{E}-03$ & $9.509041 E-03$ & & & & $3 / 16 / 9915: 03: 21$ & & & \\
\hline $2.000156 \mathrm{E}-03$ & $-9.511483 E-03$ & & & & $3 / 16 / 99$ 15:03:24 & & & \\
\hline $2.000156 \mathrm{E}-03$ & $-9.511460 E-03$ & & & & $3 / 16 / 99$ 15:03:27 & & & \\
\hline$-2.000459 E-03$ & $9.509080 \mathrm{E}-03$ & & & & $3 / 16 / 99$ 15:03:30 & & & \\
\hline$-2.000457 \mathrm{E}-03$ & $9.509078 \mathrm{E}-03$ & & & & $3 / 16 / 99$ 15:03:33 & & & \\
\hline $2.000153 E-03$ & $-9.511515 E-03$ & & & & $3 / 16 / 99$ 15:03:36 & & & \\
\hline $2.000156 \mathrm{E}-03$ & $-9.511503 E-03$ & & & & $3 / 16 / 99$ 15:03:38 & & & \\
\hline$-2.000456 \mathrm{E}-03$ & $9.509030 \mathrm{E}-03$ & & & & $3 / 16 / 99$ 15:03:41 & & & \\
\hline$-2.000455 E-03$ & $9.509052 \mathrm{E}-03$ & & & & 3/16/99 15:03:44 & & & \\
\hline 2.000153E-03 & $-9.511470 \mathrm{E}-03$ & & & & $3 / 16 / 99$ 15:03:47 & & & \\
\hline $2.000156 \mathrm{E}-03$ & $-9.511482 \mathrm{E}-03$ & & & & $3 / 16 / 9915: 03: 50$ & & & \\
\hline$-2.000452 E-03$ & $9.509051 \mathrm{E}-03$ & & & & $3 / 16 / 99$ 15:03:52 & & & \\
\hline$-2.000452 E-03$ & $9.509090 \mathrm{E}-03$ & & & & $3 / 16 / 99$ 15:03:55 & & & \\
\hline $2.000158 \mathrm{E}-03$ & $-9.511489 \mathrm{E}-03$ & & & & $3 / 16 / 99$ 15:03:58 & & & \\
\hline $2.000157 \mathrm{E}-03$ & $-9.511530 \mathrm{E}-03$ & & & & 3/16/99 15:04:01 & & & \\
\hline$-2.000452 E-03$ & $9.509014 \mathrm{E}-03$ & & & & $3 / 16 / 99$ 15:04:04 & & & \\
\hline$-2.000449 E-03$ & $9.509043 \mathrm{E}-03$ & & & & $3 / 16 / 99$ 15:04:07 & & & \\
\hline $2.000157 \mathrm{E}-03$ & $-9.511566 \mathrm{E}-03$ & & & & $3 / 16 / 99$ 15:04:09 & & & \\
\hline $2.000155 \mathrm{E}-03$ & $-9.511571 E-03$ & & & & $3 / 16 / 99$ 15:04:12 & & & \\
\hline$-2.000456 \mathrm{E}-03$ & $9.509011 \mathrm{E}-03$ & & & & $3 / 16 / 99$ 15:04:15 & & & \\
\hline$-2.000454 E-03$ & $9.509018 E-03$ & & & & $3 / 16 / 99$ 15:04:18 & & & \\
\hline $2.000155 E-03$ & $-9.511632 \mathrm{E}-03$ & & & & $3 / 16 / 99$ 15:04:21 & & & \\
\hline $2.000152 \mathrm{E}-03$ & $-9.511587 \mathrm{E}-03$ & & & & $3 / 16 / 9915: 04: 24$ & & & \\
\hline$-2.000455 E-03$ & $9.509002 E-03$ & & & & $3 / 16 / 99$ 15:04:26 & & & \\
\hline$-2.000453 \mathrm{E}-03$ & $9.509032 \mathrm{E}-03$ & & & & $3 / 16 / 99$ 15:04:29 & & & \\
\hline $2.000154 \mathrm{E}-03$ & $-9.511590 \mathrm{E}-03$ & & & & $3 / 16 / 99$ 15:04:32 & & & \\
\hline $2.000156 \mathrm{E}-03$ & $-9.511584 \mathrm{E}-03$ & & & & $3 / 16 / 99$ 15:04:35 & & & \\
\hline$-2.000455 E-03$ & $9.509066 \mathrm{E}-03$ & & & & 3/16/99 15:04:38 & & & \\
\hline$-2.000455 E-03$ & $9.509074 \mathrm{E}-03$ & & & & $3 / 16 / 9915: 04: 41$ & & & \\
\hline $2.000155 \mathrm{E}-03$ & $-9.511589 E-03$ & & & & $3 / 16 / 99$ 15:04:43 & & & \\
\hline $2.000305 E-03$ & $9.510282 \mathrm{E}-03$ & 4.754415 & $1.466538 \mathrm{E}-05$ & $1.902356 \mathrm{E}-05$ & $3 / 16 / 99$ 15:04:47 & 2366.425852 & 7.48431714 & \\
\hline & & & & & & & & \\
\hline & & & & & & & & \\
\hline
\end{tabular}




\begin{tabular}{|c|c|c|c|c|c|c|c|c|}
\hline \multirow{2}{*}{\begin{tabular}{|l|} 
Current $(A)$ \\
Sample ID $=W$
\end{tabular}} & Voltage (V) & Res.(ohms) & \multirow{2}{*}{$\frac{\text { Std. Dev. Of } \Omega}{\text { on target }=\text { approxi }}$} & Power (W) & \multirow[t]{2}{*}{ Date/time } & \multirow[t]{2}{*}{ Cernox (ohms) } & \multicolumn{2}{|c|}{ Cernox $(\mathrm{K})$ 4th order poly. } \\
\hline & Beam Energy $=1.9$ & $94 \mathrm{GeV} \quad$ Time & & mately 13 minutes & & & & \\
\hline \multicolumn{9}{|c|}{ Run aborted after 13 minutes at $1.94 \mathrm{GeV}$ due to upset conditions in LHe supply flow rate } \\
\hline & & & & & & & & \\
\hline $5.000227 E-04$ & $-2.378479 \mathrm{E}-03$ & & & & $3 / 16 / 99$ 15:05:24 & & & \\
\hline$-5.000629 E-04$ & $2.376515 \mathrm{E}-03$ & & & & $3 / 16 / 99$ 15:05:27 & & & \\
\hline$-5.000627 \mathrm{E}-04$ & $2.376502 \mathrm{E}-03$ & & & & $3 / 16 / 99$ 15:05:30 & & & \\
\hline $5.000231 \mathrm{E}-04$ & $-2.378483 \mathrm{E}-03$ & & & & $3 / 16 / 99$ 15:05:32 & & & \\
\hline $5.000230 \mathrm{E}-04$ & $-2.378488 E-03$ & & & & $3 / 16 / 99$ 15:05:35 & & & \\
\hline$-5.000628 \mathrm{E}-04$ & $2.376512 \mathrm{E}-03$ & & & & $3 / 16 / 9915: 05: 38$ & & & \\
\hline$-5.000630 \mathrm{E}-04$ & $2.376508 \mathrm{E}-03$ & & & & $3 / 16 / 99$ 15:05:41 & & & \\
\hline $5.000230 \mathrm{E}-04$ & $-2.378486 \mathrm{E}-03$ & & & & $3 / 16 / 9915: 05: 44$ & & & \\
\hline $5.000223 E-04$ & $-2.378495 E-03$ & & & & $3 / 16 / 99$ 15:05:47 & & & \\
\hline$-5.000631 E-04$ & $2.376516 \mathrm{E}-03$ & & & & $3 / 16 / 99$ 15:05:49 & & & \\
\hline$-5.000632 \mathrm{E}-04$ & $2.376522 \mathrm{E}-03$ & & & & $3 / 16 / 99$ 15:05:52 & & & \\
\hline $5.000231 \mathrm{E}-04$ & $-2.378489 E-03$ & & & & $3 / 16 / 99$ 15:05:55 & & & \\
\hline $5.000234 \mathrm{E}-04$ & $-2.378488 \mathrm{E}-03$ & & & & $3 / 16 / 9915: 05: 58$ & & & \\
\hline$-5.000633 E-04$ & $2.376507 \mathrm{E}-03$ & & & & $3 / 16 / 99$ 15:06:01 & & & \\
\hline$-5.000633 \mathrm{E}-04$ & $2.376505 E-03$ & & & & $3 / 16 / 9915: 06: 03$ & & & \\
\hline $5.000228 E-04$ & $-2.378496 \mathrm{E}-03$ & & & & $3 / 16 / 99$ 15:06:06 & & & \\
\hline $5.000227 E-04$ & $-2.378499 \mathrm{E}-03$ & & & & $3 / 16 / 99$ 15:06:09 & & & \\
\hline$-5.000639 \mathrm{E}-04$ & $2.376503 E-03$ & & & & $3 / 16 / 99$ 15:06:12 & & & \\
\hline$-5.000635 \mathrm{E}-04$ & $2.080038 \mathrm{E}+01$ & & & & $3 / 16 / 99$ 15:06:14 & & & \\
\hline $5.000234 \mathrm{E}-04$ & $-2.378659 E-03$ & & & & $3 / 16 / 99$ 15:06:17 & & & \\
\hline $5.000234 \mathrm{E}-04$ & $-2.378538 E-03$ & & & & $3 / 16 / 99$ 15:06:20 & & & \\
\hline$-5.000638 \mathrm{E}-04$ & $2.376489 E-03$ & & & & $3 / 16 / 99$ 15:06:23 & & & \\
\hline$-5.000631 E-04$ & $2.376483 \mathrm{E}-03$ & & & & $3 / 16 / 9915: 06: 26$ & & & \\
\hline $5.000232 \mathrm{E}-04$ & $-2.378516 \mathrm{E}-03$ & & & & $3 / 16 / 99$ 15:06:29 & & & \\
\hline $5.000226 \mathrm{E}-04$ & $-2.378510 \mathrm{E}-03$ & & & & $3 / 16 / 99$ 15:06:31 & & & \\
\hline$-5.000634 \mathrm{E}-04$ & $2.376504 \mathrm{E}-03$ & & & & $3 / 16 / 9915: 06: 34$ & & & \\
\hline$-5.000628 \mathrm{E}-04$ & $2.376498 \mathrm{E}-03$ & & & & $3 / 16 / 99$ 15:06:37 & & & \\
\hline $5.000234 \mathrm{E}-04$ & $-2.378525 \mathrm{E}-03$ & & & & $3 / 16 / 99$ 15:06:40 & & & \\
\hline $5.000235 E-04$ & $-2.378522 E-03$ & & & & $3 / 16 / 99 \quad 15: 06: 43$ & & & \\
\hline$-5.000629 E-04$ & $2.376484 \mathrm{E}-03$ & & & & $3 / 16 / 9915: 06: 46$ & & & \\
\hline$-5.000627 \mathrm{E}-04$ & $2.376491 \mathrm{E}-03$ & & & & $3 / 16 / 9915: 06: 48$ & & & \\
\hline $5.000232 E-04$ & $-2.378520 \mathrm{E}-03$ & & & & $3 / 16 / 9915: 06: 51$ & & & \\
\hline $5.000233 \mathrm{E}-04$ & $-2.378521 E-03$ & & & & $3 / 16 / 99$ 15:06:54 & & & \\
\hline$-5.000631 E-04$ & $2.376498 \mathrm{E}-03$ & & & & $3 / 16 / 99$ 15:06:57 & & & \\
\hline$-5.000632 E-04$ & $2.376490 \mathrm{E}-03$ & & & & $3 / 16 / 99$ 15:07:00 & & & \\
\hline $5.000231 E-04$ & $-2.378506 \mathrm{E}-03$ & & & & $3 / 16 / 99$ 15:07:02 & & & \\
\hline $5.000231 E-04$ & $-2.378519 \mathrm{E}-03$ & & & & $3 / 16 / 9915: 07: 05$ & & & \\
\hline$-5.000634 E-04$ & $2.376495 \mathrm{E}-03$ & & & & $3 / 16 / 9915: 07: 08$ & & & \\
\hline$-5.000635 \mathrm{E}-04$ & $2.376486 \mathrm{E}-03$ & & & & $3 / 16 / 99$ 15:07:11 & & & \\
\hline $5.000230 E-04$ & $-2.378514 E-03$ & & & & $3 / 16 / 9915: 07: 14$ & & & \\
\hline $5.000431 E-04$ & $5.223275 \mathrm{E}-01$ & 1044.564130 & $4.532425 \mathrm{E}+03$ & $1.188853 \mathrm{E}-06$ & $3 / 16 / 99$ 15:07:18 & 2341.281487 & 7.539148779 & \\
\hline & & & & & & & & \\
\hline
\end{tabular}


Tungsten.xls

\begin{tabular}{|c|c|c|c|c|c|c|c|c|}
\hline \multirow{2}{*}{\begin{tabular}{|l} 
Current $(A)$ \\
Sample ID=W
\end{tabular}} & Voltage (V) & Res.(ohms) & Std. Dev. Of $\Omega$ & Power (W) & \multirow[t]{2}{*}{ Date/time } & \multirow[t]{2}{*}{ Cernox (ohms) } & \multicolumn{2}{|c|}{ Cernox (K) 4th order poly. } \\
\hline & Beam Energy $=1.9$ & $94 \mathrm{GeV}$ & on target= approxin & mately 13 minutes & & & & \\
\hline \multicolumn{9}{|c|}{ Run aborted after 13 minutes at $1.94 \mathrm{GeV}$ due to upset conditions in LHe supply flow rate } \\
\hline & & & & & & & & \\
\hline $5.000223 \mathrm{E}-04$ & $-2.378523 E-03$ & & & & $3 / 16 / 99$ 15:07:26 & & & \\
\hline$-5.000638 E-04$ & $2.376511 \mathrm{E}-03$ & & & & $3 / 16 / 99$ 15:07:29 & & & \\
\hline$-5.000635 E-04$ & $2.376507 \mathrm{E}-03$ & & & & $3 / 16 / 99$ 15:07:32 & & & \\
\hline $5.000223 E-04$ & $-2.378522 E-03$ & & & & $3 / 16 / 99$ 15:07:35 & & & \\
\hline $5.000225 E-04$ & $-2.378507 E-03$ & & & & $3 / 16 / 9915: 07: 38$ & & & \\
\hline$-5.000635 \mathrm{E}-04$ & $2.376501 E-03$ & & & & $3 / 16 / 9915: 07: 40$ & & & \\
\hline$-5.000635 E-04$ & $2.376503 \mathrm{E}-03$ & & & & $3 / 16 / 9915: 07: 43$ & & & \\
\hline $5.000226 \mathrm{E}-04$ & $-2.378523 E-03$ & & & & $3 / 16 / 9915: 07: 46$ & & & \\
\hline $5.000226 \mathrm{E}-04$ & $-2.378526 E-03$ & & & & $3 / 16 / 99$ 15:07:49 & & & \\
\hline$-5.000642 \mathrm{E}-04$ & $2.376505 \mathrm{E}-03$ & & & & $3 / 16 / 99$ 15:07:52 & & & \\
\hline$-5.000642 E-04$ & $2.376501 E-03$ & & & & $3 / 16 / 99$ 15:07:54 & & & \\
\hline $5.000227 \mathrm{E}-04$ & $-2.378524 \mathrm{E}-03$ & & & & $3 / 16 / 99$ 15:07:57 & & & \\
\hline $5.000224 \mathrm{E}-04$ & $-2.378519 E-03$ & & & & 3/16/99 15:08:00 & & & \\
\hline$-5.000639 E-04$ & $2.376499 \mathrm{E}-03$ & & & & $3 / 16 / 9915: 08: 03$ & & & \\
\hline$-5.000643 E-04$ & $2.376504 \mathrm{E}-03$ & & & & $3 / 16 / 99$ 15:08:06 & & & \\
\hline $5.000224 \mathrm{E}-04$ & $-2.378520 \mathrm{E}-03$ & & & & $3 / 16 / 99$ 15:08:09 & & & \\
\hline $5.000227 \mathrm{E}-04$ & $-2.378523 \mathrm{E}-03$ & & & & $3 / 16 / 99$ 15:08:11 & & & \\
\hline$-5.000645 E-04$ & $2.376503 E-03$ & & & & 3/16/99 15:08:14 & & & \\
\hline$-5.000648 E-04$ & $2.376510 \mathrm{E}-03$ & & & & $3 / 16 / 99$ 15:08:17 & & & \\
\hline $5.000222 \mathrm{E}-04$ & $-2.378513 E-03$ & & & & $3 / 16 / 99$ 15:08:20 & & & \\
\hline $5.000224 \mathrm{E}-04$ & $-2.378534 E-03$ & & & & $3 / 16 / 99$ 15:08:23 & & & \\
\hline$-5.000648 E-04$ & $2.376510 \mathrm{E}-03$ & & & & $3 / 16 / 99$ 15:08:26 & & & \\
\hline$-5.000645 \mathrm{E}-04$ & $2.376500 \mathrm{E}-03$ & & & & $3 / 16 / 99$ 15:08:28 & & & \\
\hline $5.000231 E-04$ & $-2.378532 E-03$ & & & & $3 / 16 / 99$ 15:08:31 & & & \\
\hline $5.000231 E-04$ & $-2.378532 E-03$ & & & & $3 / 16 / 99$ 15:08:34 & & & \\
\hline$-5.000648 \mathrm{E}-04$ & $2.376504 \mathrm{E}-03$ & & & & $3 / 16 / 9915: 08: 37$ & & & \\
\hline$-5.000648 \mathrm{E}-04$ & $2.376504 \mathrm{E}-03$ & & & & $3 / 16 / 9915: 08: 40$ & & & \\
\hline $5.000231 \mathrm{E}-04$ & $-2.378539 E-03$ & & & & $3 / 16 / 9915: 08: 43$ & & & \\
\hline $5.000232 E-04$ & $-2.378540 \mathrm{E}-03$ & & & & $3 / 16 / 9915: 08: 45$ & & & \\
\hline$-5.000642 \mathrm{E}-04$ & $2.376502 E-03$ & & & & $3 / 16 / 9915: 08: 48$ & & & \\
\hline$-5.000643 \mathrm{E}-04$ & $2.376499 \mathrm{E}-03$ & & & & $3 / 16 / 9915: 08: 51$ & & & \\
\hline $5.000228 \mathrm{E}-04$ & $-2.378533 \mathrm{E}-03$ & & & & $3 / 16 / 9915: 08: 54$ & & & \\
\hline $5.000226 \mathrm{E}-04$ & $-2.378540 \mathrm{E}-03$ & & & & $3 / 16 / 9915: 08: 57$ & & & \\
\hline$-5.000644 E-04$ & $2.376541 \mathrm{E}-03$ & & & & $3 / 16 / 99$ 15:09:00 & & & \\
\hline$-5.000643 E-04$ & $2.376445 E-03$ & & & & $3 / 16 / 99$ 15:09:02 & & & \\
\hline $5.000230 \mathrm{E}-04$ & $-2.378603 \mathrm{E}-03$ & & & & $3 / 16 / 99$ 15:09:05 & & & \\
\hline $5.000235 E-04$ & $-2.378519 \mathrm{E}-03$ & & & & $3 / 16 / 9915: 09: 08$ & & & \\
\hline$-5.000640 \mathrm{E}-04$ & $2.376498 \mathrm{E}-03$ & & & & $3 / 16 / 99$ 15:09:11 & & & \\
\hline$-5.000639 E-04$ & $2.376463 \mathrm{E}-03$ & & & & $3 / 16 / 99$ 15:09:14 & & & \\
\hline $5.000233 \mathrm{E}-04$ & $-2.378598 \mathrm{E}-03$ & & & & $3 / 16 / 99$ 15:09:16 & & & \\
\hline $5.000435 E-04$ & $2.377517 \mathrm{E}-03$ & 4.754621 & 1.515653E-05 & 1.188869E-06 & $3 / 16 / 9915: 09: 21$ & 2324.625839 & 7.576108456 & \\
\hline & & & & & & & & \\
\hline
\end{tabular}


Tungsten.xls

\begin{tabular}{|c|c|c|c|c|c|c|c|c|}
\hline Current $(A)$ & Voltage (V) & Res.(ohms) & Std. Dev. Of $\Omega$ & Power (W) & Date/time & Cernox (ohms) & \multicolumn{2}{|c|}{ Cernox (K) 4th order poly } \\
\hline Sample ID=W & Beam Energy $=0$ & \multicolumn{2}{|c|}{ Time on target $=0$} & & & & & \\
\hline \multicolumn{9}{|c|}{ Room Temperature measurement between 1.94 and $1.1 \mathrm{GeV}$} \\
\hline & & & & & & & & \\
\hline 5.000253E-05 & $-3.064542 \mathrm{E}-03$ & & & & $3 / 16 / 99$ 16:22:35 & & & \\
\hline$-5.000599 \mathrm{E}-05$ & 2.892743E-03 & & & & $3 / 16 / 99$ 16:22:38 & & & \\
\hline$-5.000603 E-05$ & $2.893095 \mathrm{E}-03$ & & & & $3 / 16 / 9916: 22: 41$ & & & \\
\hline $5.000260 \mathrm{E}-05$ & $-3.065153 \mathrm{E}-03$ & & & & $3 / 16 / 99$ 16:22:44 & & & \\
\hline $5.000259 \mathrm{E}-05$ & $-3.065352 E-03$ & & & & $3 / 16 / 9916: 22: 47$ & & & \\
\hline$-5.000601 \mathrm{E}-05$ & 2.894130E-03 & & & & $3 / 16 / 99$ 16:22:49 & & & \\
\hline$-5.000599 \mathrm{E}-05$ & $2.894475 \mathrm{E}-03$ & & & & $3 / 16 / 9916: 22: 52$ & & & \\
\hline $5.000254 \mathrm{E}-05$ & $-3.065969 \mathrm{E}-03$ & & & & $3 / 16 / 9916: 22: 55$ & & & \\
\hline $5.000250 \mathrm{E}-05$ & $-3.066184 \mathrm{E}-03$ & & & & $3 / 16 / 9916: 22: 58$ & & & \\
\hline$-5.000603 \mathrm{E}-05$ & $2.895554 \mathrm{E}-03$ & & & & 3/16/99 16:23:01 & & & \\
\hline$-5.000598 \mathrm{E}-05$ & $2.895897 \mathrm{E}-03$ & & & & $3 / 16 / 99$ 16:23:04 & & & \\
\hline $5.000253 \mathrm{E}-05$ & $-3.066820 \mathrm{E}-03$ & & & & $3 / 16 / 99$ 16:23:06 & & & \\
\hline $5.000254 \mathrm{E}-05$ & $-3.067068 \mathrm{E}-03$ & & & & $3 / 16 / 99$ 16:23:09 & & & \\
\hline$-5.000605 E-05$ & $2.896983 \mathrm{E}-03$ & & & & $3 / 16 / 9916: 23: 12$ & & & \\
\hline$-5.000603 E-05$ & $2.897339 \mathrm{E}-03$ & & & & $3 / 16 / 99$ 16:23:15 & & & \\
\hline $5.000259 \mathrm{E}-05$ & $-3.067681 \mathrm{E}-03$ & & & & $3 / 16 / 99$ 16:23:18 & & & \\
\hline $5.000256 \mathrm{E}-05$ & $-3.067937 E-03$ & & & & $3 / 16 / 9916: 23: 21$ & & & \\
\hline$-5.000599 \mathrm{E}-05$ & $2.898436 \mathrm{E}-03$ & & & & $3 / 16 / 99$ 16:23:23 & & & \\
\hline$-5.000604 E-05$ & $2.898785 \mathrm{E}-03$ & & & & $3 / 16 / 9916: 23: 26$ & & & \\
\hline $5.000258 \mathrm{E}-05$ & $-3.068605 \mathrm{E}-03$ & & & & $3 / 16 / 99$ 16:23:29 & & & \\
\hline $5.000429 E-05$ & $2.981137 \mathrm{E}-03$ & 59.617638 & $3.261179 \mathrm{E}-02$ & 1.491976E-07 & $3 / 16 / 9916: 23: 33$ & 60.26658068 & 292.2257381 & \\
\hline & & & & & & & & \\
\hline
\end{tabular}


Tungsten.xls

\begin{tabular}{|c|c|c|c|c|c|c|c|c|}
\hline Current $(\mathrm{A})$ & Voltage (V) & Res.(ohms) & Std. Dev. Of $\Omega$ & Power (W) & Date/time & Cernox (ohms) & Cernox $(\mathrm{K})$ 4th ord & er poly. \\
\hline Sample ID $=W$ & Beam Energy $=0$ & Time on target & $=0$ & & & & & \\
\hline Room Temperature & measurement betv & ween 1.94 and $1.1 \mathrm{C}$ & $\mathrm{GeV}$ & & & & & \\
\hline & & & & & & & & \\
\hline $5.000228 \mathrm{E}-04$ & $-2.995831 \mathrm{E}-02$ & & & & $3 / 16 / 99$ 16:24:05 & & & \\
\hline$-5.000726 E-04$ & $2.979495 \mathrm{E}-02$ & & & & $3 / 16 / 99$ 16:24:08 & & & \\
\hline$-5.000721 E-04$ & $2.979826 \mathrm{E}-02$ & & & & $3 / 16 / 99$ 16:24:11 & & & \\
\hline $5.000230 \mathrm{E}-04$ & $-2.996753 \mathrm{E}-02$ & & & & $3 / 16 / 99$ 16:24:13 & & & \\
\hline $5.000231 \mathrm{E}-04$ & $-2.997062 \mathrm{E}-02$ & & & & $3 / 16 / 99 \quad 16: 24: 16$ & & & \\
\hline$-5.000728 E-04$ & $2.980792 E-02$ & & & & $3 / 16 / 9916: 24: 19$ & & & \\
\hline$-5.000730 \mathrm{E}-04$ & $2.981113 \mathrm{E}-02$ & & & & $3 / 16 / 99 \quad 16: 24: 22$ & & & \\
\hline $5.000224 \mathrm{E}-04$ & $-2.998000 \mathrm{E}-02$ & & & & $3 / 16 / 99 \quad 16: 24: 25$ & & & \\
\hline $5.000221 \mathrm{E}-04$ & $-2.998309 \mathrm{E}-02$ & & & & $3 / 16 / 9916: 24: 27$ & & & \\
\hline$-5000734 \mathrm{E}-04$ & $2.982120 \mathrm{E}-02$ & & & & $3 / 16 / 99$ 16:24:30 & & & \\
\hline$-5.000735 E-04$ & $2.982454 \mathrm{E}-02$ & & & & $3 / 16 / 99$ 16:24:33 & & & \\
\hline $5.000222 \mathrm{E}-04$ & $-2.999291 \mathrm{E}-02$ & & & & $3 / 16 / 99$ 16:24:36 & & & \\
\hline $5.000224 \mathrm{E}-04$ & $-2.999612 \mathrm{E}-02$ & & & & $3 / 16 / 99$ 16:24:39 & & & \\
\hline$-5.000742 E-04$ & $2.983458 E-02$ & & & & $3 / 16 / 99$ 16:24:42 & & & \\
\hline$-5.000741 E-04$ & $2.983792 \mathrm{E}-02$ & & & & $3 / 16 / 99$ 16:24:44 & & & \\
\hline $5.000232 \mathrm{E}-04$ & $-3.000585 E-02$ & & & & $3 / 16 / 99$ 16:24:47 & & & \\
\hline $5.000227 E-04$ & $-3.000901 E-02$ & & & & $3 / 16 / 99$ 16:24:50 & & & \\
\hline$-5.000732 \mathrm{E}-04$ & $2.984792 \mathrm{E}-02$ & & & & $3 / 16 / 99$ 16:24:53 & & & \\
\hline$-5.000735 \mathrm{E}-04$ & $2.985133 \mathrm{E}-02$ & & & & $3 / 16 / 99$ 16:24:56 & & & \\
\hline $5.000225 \mathrm{E}-04$ & $-3.001881 E-02$ & & & & $3 / 16 / 99$ 16:24:59 & & & \\
\hline 5.000479E-04 & $2.990560 \mathrm{E}-02$ & 59.805468 & $3.736468 \mathrm{E}-02$ & 1.496897E-05 & $3 / 16 / 9916: 25: 03$ & 60.12281769 & 293.0351358 & \\
\hline & & & & & & & & \\
\hline & & & & & & & & \\
\hline
\end{tabular}


Tungsten.xls

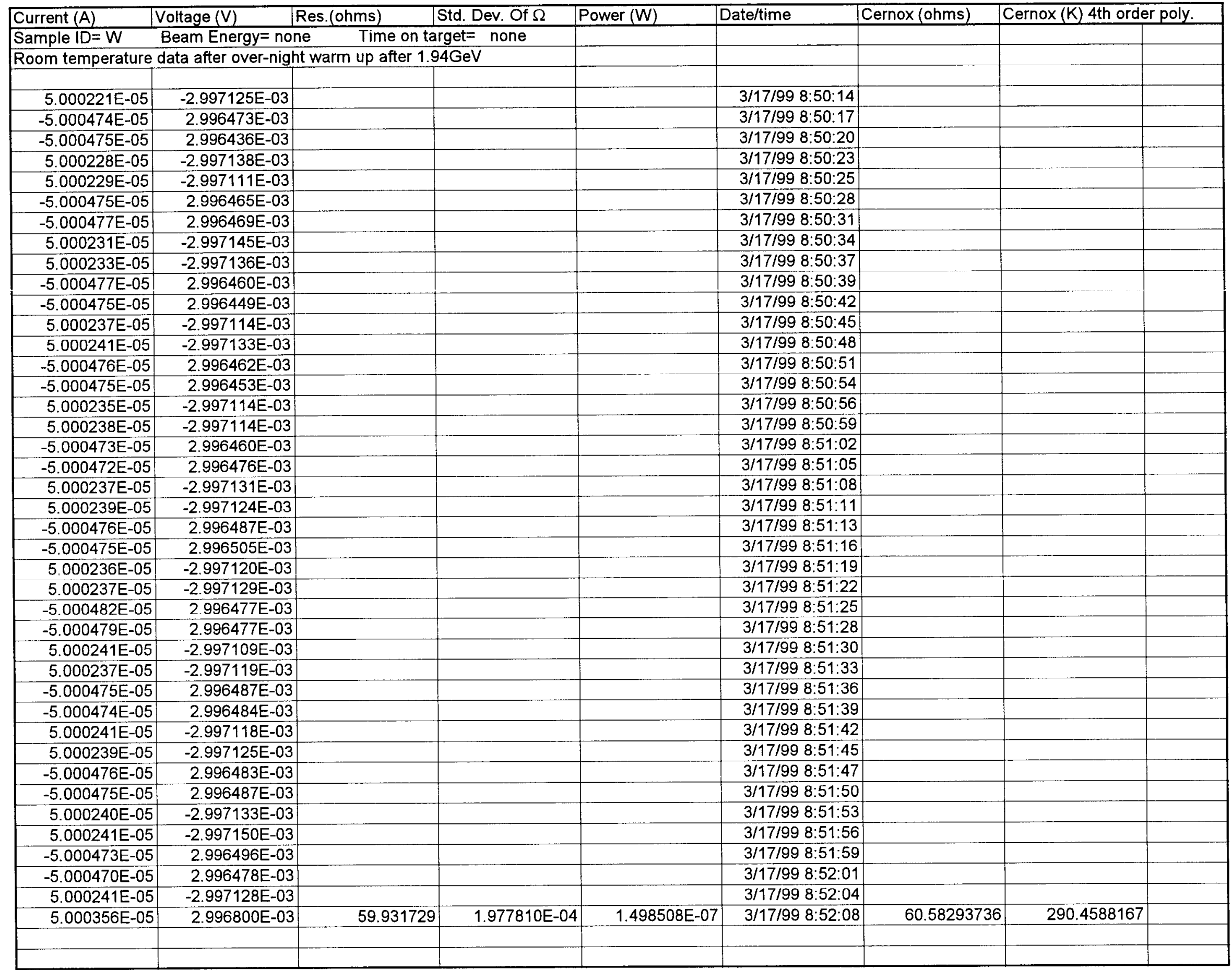


Tungsten.xls

\begin{tabular}{|c|c|c|c|c|c|c|c|c|}
\hline Current $(\mathrm{A})$ & Voltage (V) & Res.(ohms) & Std. Dev. Of $\Omega$ & Power (W) & Date/time & Cernox (ohms) & \multicolumn{2}{|c|}{ Cernox $(\mathrm{K})$ 4th order poly. } \\
\hline Sample ID $=W$ & \multicolumn{3}{|c|}{ Beam Energy $=$ none $\quad$ Time on target $=$ none } & & & & & \\
\hline \multicolumn{9}{|c|}{ Room temperature data after over-night warm up after $1.94 \mathrm{GeV}$} \\
\hline & & & & & & & & \\
\hline $5.000204 \mathrm{E}-04$ & $-2.996422 E-02$ & & & & $3 / 17 / 998: 53: 30$ & & & \\
\hline$-5.000606 \mathrm{E}-04$ & $2.996351 \mathrm{E}-02$ & & & & $3 / 17 / 998: 53: 33$ & & & \\
\hline$-5.000606 \mathrm{E}-04$ & $2.996357 \mathrm{E}-02$ & & & & 3/17/99 8:53:36 & & & \\
\hline $5.000208 \mathrm{E}-04$ & $-2.996435 \mathrm{E}-02$ & & & & $3 / 17 / 998: 53: 38$ & & & \\
\hline $5.000201 E-04$ & $-2.996434 \mathrm{E}-02$ & & & & $3 / 17 / 998: 53: 41$ & & & \\
\hline$-5.000604 \mathrm{E}-04$ & $2.996361 \mathrm{E}-02$ & & & & 3/17/99 8:53:44 & & & \\
\hline$-5.000608 \mathrm{E}-04$ & 2.996357E-02 & & & & $3 / 17 / 998: 53: 47$ & & & \\
\hline $5.000203 E-04$ & $-2.996444 E-02$ & & & & $3 / 17 / 998: 53: 50$ & & & \\
\hline $5.000204 E-04$ & $-2.996442 E-02$ & & & & $3 / 17 / 998: 53: 53$ & & & \\
\hline$-5000609 E-04$ & 2.996353E-02 & & & & $3 / 17 / 998: 53: 55$ & & & \\
\hline$-5.000609 E-04$ & $2.996352 \mathrm{E}-02$ & & & & $3 / 17 / 998: 53: 58$ & & & \\
\hline 5.000205E-04 & $-2.996448 E-02$ & & & & $3 / 17 / 998: 54: 01$ & & & \\
\hline $5.000202 E-04$ & $-2.996457 \mathrm{E}-02$ & & & & $3 / 17 / 998: 54: 04$ & & & \\
\hline$-5.000609 E-04$ & $2.996366 \mathrm{E}-02$ & & & & $3 / 17 / 998: 54: 07$ & & & \\
\hline$-5.000610 \mathrm{E}-04$ & $2.996364 \mathrm{E}-02$ & & & & $3 / 17 / 998: 54: 09$ & & & \\
\hline $5.000206 \mathrm{E}-04$ & $-2.996455 \mathrm{E}-02$ & & & & $3 / 17 / 998: 54: 12$ & & & \\
\hline $5.000208 \mathrm{E}-04$ & $-2.996458 \mathrm{E}-02$ & & & & $3 / 17 / 998: 54: 15$ & & & \\
\hline$-5.000609 \mathrm{E}-04$ & $2.996368 \mathrm{E}-02$ & & & & $3 / 17 / 998: 54: 18$ & & & \\
\hline$-5.000611 E-04$ & $2.996364 \mathrm{E}-02$ & & & & $3 / 17 / 998: 54: 21$ & & & \\
\hline $5.000200 \mathrm{E}-04$ & $-2.996458 \mathrm{E}-02$ & & & & $3 / 17 / 998: 54: 24$ & & & \\
\hline $5.000206 \mathrm{E}-04$ & $-2.996460 \mathrm{E}-02$ & & & & $3 / 17 / 998: 54: 26$ & & & \\
\hline$-5.000611 E-04$ & $2.996373 \mathrm{E}-02$ & & & & $3 / 17 / 998: 54: 29$ & & & \\
\hline$-5.000606 \mathrm{E}-04$ & $2.996372 \mathrm{E}-02$ & & & & $3 / 17 / 998: 54: 32$ & & & \\
\hline $5.000213 E-04$ & $-2.996469 \mathrm{E}-02$ & & & & $3 / 17 / 998: 54: 35$ & & & \\
\hline $5.000213 E-04$ & $-2.996473 E-02$ & & & & $3 / 17 / 998: 54: 38$ & & & \\
\hline$-5.000604 E-04$ & $2.996364 \mathrm{E}-02$ & & & & $3 / 17 / 998: 54: 40$ & & & \\
\hline$-5.000604 E-04$ & $2.996375 \mathrm{E}-02$ & & & & $3 / 17 / 998: 54: 43$ & & & \\
\hline $5.000211 \mathrm{E}-04$ & $-2.996473 \mathrm{E}-02$ & & & & $3 / 17 / 998: 54: 46$ & & & \\
\hline $5.000213 E-04$ & $-2.996477 \mathrm{E}-02$ & & & & $3 / 17 / 998: 54: 49$ & & & \\
\hline$-5.000611 \mathrm{E}-04$ & $2.996375 \bar{E}-02$ & & & & $3 / 17 / 998: 54: 52$ & & & \\
\hline$-5.000609 \mathrm{E}-04$ & $2.996374 \mathrm{E}-02$ & & & & $3 / 17 / 998: 54: 55$ & & & \\
\hline $5.000205 E-04$ & $-2.996474 \mathrm{E}-02$ & & & & $3 / 17 / 998: 54: 57$ & & & \\
\hline $5.000207 \mathrm{E}-04$ & $-2.996473 E-02$ & & & & $3 / 17 / 998: 55: 00$ & & & \\
\hline$-5.000610 \mathrm{E}-04$ & $2.996376 \mathrm{E}-02$ & & & & $3 / 17 / 998: 55: 03$ & & & \\
\hline$-5.000614 \mathrm{E}-04$ & $2.996378 \mathrm{E}-02$ & & & & 3/17/99 8:55:06 & & & \\
\hline $5.000208 \mathrm{E}-04$ & $-2.996472 \mathrm{E}-02$ & & & & $3 / 17 / 998: 55: 09$ & & & \\
\hline $5.000206 \mathrm{E}-04$ & $-2.996477 \mathrm{E}-02$ & & & & $3 / 17 / 998: 55: 12$ & & & \\
\hline$-5.000618 \mathrm{E}-04$ & $2.996378 \mathrm{E}-02$ & & & & $3 / 17 / 998: 55: 14$ & & & \\
\hline$-5.000617 \mathrm{E}-04$ & $2.996388 \mathrm{E}-02$ & & & & $3 / 17 / 998: 55: 17$ & & & \\
\hline $5.000206 \mathrm{E}-04$ & $-2.996469 \mathrm{E}-02$ & & & & $3 / 17 / 998: 55: 20$ & & & \\
\hline $5.000408 \mathrm{E}-04$ & $2.996413 \mathrm{E}-02$ & 59.923370 & $2.241765 \mathrm{E}-04$ & 1.498337E-05 & $3 / 17 / 998: 55: 24$ & 60.57488402 & 290.5035561 & \\
\hline & & & & & & & & \\
\hline
\end{tabular}


Tungsten.xis

\begin{tabular}{|c|c|c|c|c|c|c|c|c|}
\hline \multirow{3}{*}{\begin{tabular}{|l|} 
Current $(A)$ \\
Sample $I D=W$
\end{tabular}} & Voltage (V) & Res.(ohms) & Std. Dev. Of $\Omega$ & \multirow[t]{2}{*}{ Power $(W)$} & \multirow[t]{2}{*}{ Date/time } & \multirow[t]{2}{*}{ Cernox (ohms) } & \multicolumn{2}{|c|}{ Cernox $(K)$ 4th order poly. } \\
\hline & Beam Energy $=\mathrm{pr}$ & ore-1.1 GeV & me on target $=0$ & & & & & \\
\hline & \multicolumn{8}{|c|}{ This is the cold data acquisition at $4.72 \mathrm{~K}$ prior to irradiation at $1.1 \mathrm{GeV}$} \\
\hline & & & & & & & & \\
\hline $5.000204 \mathrm{E}-04$ & $-2.362694 \mathrm{E}-03$ & & & & $3 / 17 / 99$ 10:16:47 & & & \\
\hline$-5.000747 E-04$ & $2.360482 \mathrm{E}-03$ & & & & $3 / 17 / 99$ 10:16:49 & & & \\
\hline$-5.000743 \mathrm{E}-04$ & $2.360474 \mathrm{E}-03$ & & & & $3 / 17 / 9910: 16: 52$ & & & \\
\hline $5.000212 E-04$ & $-2.362699 \mathrm{E}-03$ & & & & 3/17/99 10:16:55 & & & \\
\hline $5.000215 E-04$ & $-2.362694 \mathrm{E}-03$ & & & & $3 / 17 / 99$ 10:16:58 & & & \\
\hline$-5.000738 E-04$ & $2.360481 \mathrm{E}-03$ & & & & $3 / 17 / 9910: 17: 01$ & & & \\
\hline$-5.000741 E-04$ & $2.360485 \mathrm{E}-03$ & & & & $3 / 17 / 99$ 10:17:04 & & & \\
\hline $5.000209 E-04$ & $-2.362722 \mathrm{E}-03$ & & & & $3 / 17 / 99$ 10:17:06 & & & \\
\hline $5.000210 \mathrm{E}-04$ & $-2.362696 \mathrm{E}-03$ & & & & $3 / 17 / 99$ 10:17:09 & & & \\
\hline$-5.000746 \mathrm{E}-04$ & $2.360482 E-03$ & & & & $3 / 17 / 99$ 10:17:12 & & & \\
\hline$-5.000740 \mathrm{E}-04$ & $2.360508 E-03$ & & & & $3 / 17 / 99$ 10:17:15 & & & \\
\hline $5.000211 \mathrm{E}-04$ & $-2.362695 \mathrm{E}-03$ & & & & $3 / 17 / 99$ 10:17:18 & & & \\
\hline $5.000214 \mathrm{E}-04$ & $-2.362675 \mathrm{E}-03$ & & & & $3 / 17 / 99$ 10:17:20 & & & \\
\hline$-5.000737 E-04$ & $2.360508 \mathrm{E}-03$ & & & & $3 / 17 / 9910: 17: 23$ & & & \\
\hline$-5.000736 \mathrm{E}-04$ & $2.360522 \mathrm{E}-03$ & & & & $3 / 17 / 99 \quad 10: 17: 26$ & & & \\
\hline $5.000209 \mathrm{E}-04$ & $-2.362686 \mathrm{E}-03$ & & & & $3 / 17 / 9910: 17: 29$ & & & \\
\hline $5.000212 E-04$ & $-2.362686 \mathrm{E}-03$ & & & & $3 / 17 / 99$ 10:17:32 & & & \\
\hline$-5.000742 \mathrm{E}-04$ & $2.360526 \mathrm{E}-03$ & & & & $3 / 17 / 99$ 10:17:35 & & & \\
\hline$-5.000745 E-04$ & $2.360536 \mathrm{E}-03$ & & & & $3 / 17 / 99 \quad 10: 17: 37$ & & & \\
\hline $5.000207 E-04$ & $-2.362648 E-03$ & & & & $3 / 17 / 99 \quad 10: 17: 40$ & & & \\
\hline $5.000214 \mathrm{E}-04$ & $-2.362657 E-03$ & & & & $3 / 17 / 99$ 10:17:43 & & & \\
\hline$-5.000744 \mathrm{E}-04$ & $2.360528 \mathrm{E}-03$ & & & & $3 / 17 / 99$ 10:17:46 & & & \\
\hline$-5.000738 \mathrm{E}-04$ & $2.360509 \mathrm{E}-03$ & & & & $3 / 17 / 99 \quad 10: 17: 49$ & & & \\
\hline $5.000218 \mathrm{E}-04$ & $-2.362657 \mathrm{E}-03$ & & & & $3 / 17 / 99$ 10:17:52 & & & \\
\hline $5.000218 \mathrm{E}-04$ & $-2.362684 \mathrm{E}-03$ & & & & $3 / 17 / 99$ 10:17:54 & & & \\
\hline$-5.000739 E-04$ & $2.360527 \mathrm{E}-03$ & & & & $3 / 17 / 99$ 10:17:57 & & & \\
\hline$-5.000737 \mathrm{E}-04$ & $2.360528 \mathrm{E}-03$ & & & & 3/17/99 10:18:00 & & & \\
\hline $5.000213 E-04$ & $-2.362668 \mathrm{E}-03$ & & & & $3 / 17 / 99 \quad 10: 18: 03$ & & & \\
\hline $5.000213 \mathrm{E}-04$ & $-2.362676 \mathrm{E}-03$ & & & & $3 / 17 / 99 \quad 10: 18: 06$ & & & \\
\hline$-5.000744 E-04$ & $2.360529 \mathrm{E}-03$ & & & & $3 / 17 / 99$ 10:18:09 & & & \\
\hline$-5.000744 \mathrm{E}-04$ & $2.360542 \mathrm{E}-03$ & & & & $3 / 17 / 99$ 10:18:11 & & & \\
\hline $5.000216 \mathrm{E}-04$ & $-2.362674 \mathrm{E}-03$ & & & & $3 / 17 / 9910: 18: 14$ & & & \\
\hline $5.000217 \mathrm{E}-04$ & $-2.362652 E-03$ & & & & $3 / 17 / 99$ 10:18:17 & & & \\
\hline$-5.000745 E-04$ & $2.360534 \mathrm{E}-03$ & & & & $3 / 17 / 99$ 10:18:20 & & & \\
\hline$-5.000746 E-04$ & $2.360528 \mathrm{E}-03$ & & & & $3 / 17 / 99$ 10:18:23 & & & \\
\hline $5.000210 \mathrm{E}-04$ & $-2.362647 \mathrm{E}-03$ & & & & $3 / 17 / 99$ 10:18:26 & & & \\
\hline $5.000209 \mathrm{E}-04$ & $-2.362687 \mathrm{E}-03$ & & & & $3 / 17 / 9910: 18: 28$ & & & \\
\hline$-5.000746 \mathrm{E}-04$ & $2.360555 \mathrm{E}-03$ & & & & $3 / 17 / 99$ 10:18:31 & & & \\
\hline$-5.000746 \mathrm{E}-04$ & $2.360541 \mathrm{E}-03$ & & & & $3 / 17 / 9910: 18: 34$ & & & \\
\hline $5.000208 E-04$ & $-2.362627 \mathrm{E}-03$ & & & & $3 / 17 / 99$ 10:18:37 & & & \\
\hline $5.000477 \mathrm{E}-04$ & $2.361596 \mathrm{E}-03$ & 4.722742 & $1.955632 \mathrm{E}-05$ & 1.180905E-06 & $3 / 17 / 99$ 10:18:41 & 4976.237834 & 4.726870248 & \\
\hline & & & & & & & & \\
\hline
\end{tabular}


Tungsten.xls

\begin{tabular}{|c|c|c|c|c|c|c|c|c|}
\hline Current (A) & Voltage $(\mathrm{V})$ & Res.(ohms) & Std. Dev. Of $\Omega$ & Power (W) & Date/time & Cernox (ohms) & Cernox $(\mathrm{K})$ 4th orde & er poly. \\
\hline Sample ID $=W$ & Beam Energy $=p$ & re-1.1 GeV & ne on target $=0$ & & & & & \\
\hline This is the cold data & ta acquisition at 4.72 & $2 \mathrm{~K}$ prior to irradiatio & on at $1.1 \mathrm{GeV}$ & & & & & \\
\hline & & & & & & & & \\
\hline $9.999648 \mathrm{E}-04$ & $-4.723672 \mathrm{E}-03$ & & & & 3/17/99 10:19:17 & & & \\
\hline$-1.000002 E-03$ & $4.721646 \mathrm{E}-03$ & & & & $3 / 17 / 99$ 10:19:20 & & & \\
\hline$-1.000001 E-03$ & $4.721624 \mathrm{E}-03$ & & & & $3 / 17 / 99$ 10:19:22 & & & \\
\hline $9.999657 \mathrm{E}-04$ & $-4.723698 \mathrm{E}-03$ & & & & $3 / 17 / 99$ 10:19:25 & & & \\
\hline $9.999650 \mathrm{E}-04$ & $-4.723687 \mathrm{E}-03$ & & & & $3 / 17 / 99$ 10:19:28 & & & \\
\hline$-1.000003 E-03$ & 4.721618E-03 & & & & $3 / 17 / 99$ 10:19:31 & & & \\
\hline$-1.000003 E-03$ & $4.721638 \mathrm{E}-03$ & & & & $3 / 17 / 99$ 10:19:34 & & & \\
\hline $9.999648 E-04$ & $-4.723713 \mathrm{E}-03$ & & & & $3 / 17 / 99$ 10:19:36 & & & \\
\hline $9.999640 \mathrm{E}-04$ & $-4.723710 \mathrm{E}-03$ & & & & $3 / 17 / 99$ 10:19:39 & & & \\
\hline$-1.000003 \mathrm{E}-03$ & 4.721605E-03 & & & & $3 / 17 / 99$ 10:19:42 & & & \\
\hline$-1.000003 E-03$ & $4.721603 \mathrm{E}-03$ & & & & $3 / 17 / 99 \quad 10: 19: 45$ & & & \\
\hline $9.999651 \mathrm{E}-04$ & $-4.723722 \mathrm{E}-03$ & & & & $3 / 17 / 99$ 10:19:48 & & & \\
\hline $9.999650 \mathrm{E}-04$ & $-4.723709 \mathrm{E}-03$ & & & & $3 / 17 / 99$ 10:19:51 & & & \\
\hline$-1.000002 E-03$ & 4.721571E-03 & & & & $3 / 17 / 99$ 10:19:53 & & & \\
\hline$-1.000002 E-03$ & $4.721616 \mathrm{E}-03$ & & & & $3 / 17 / 99 \quad 10: 19: 56$ & & & \\
\hline 9.999657E-04 & $-4.723704 E-03$ & & & & $3 / 17 / 99$ 10:19:59 & & & \\
\hline 9.999651E-04 & $-4.723709 \mathrm{E}-03$ & & & & $3 / 17 / 99$ 10:20:02 & & & \\
\hline$-1.000003 E-03$ & $4.721604 \mathrm{E}-03$ & & & & $3 / 17 / 99$ 10:20:05 & & & \\
\hline$-1.000002 E-03$ & 4.721613E-03 & & & & $3 / 17 / 99 \quad 10: 20: 08$ & & & \\
\hline $9.999662 \mathrm{E}-04$ & $-4.723690 \mathrm{E}-03$ & & & & 3/17/99 10:20:10 & & & \\
\hline $9.999652 E-04$ & $-4.723715 \mathrm{E}-03$ & & & & $3 / 17 / 99$ 10:20:13 & & & \\
\hline$-1.000003 \mathrm{E}-03$ & $4.721611 \mathrm{E}-03$ & & & & $3 / 17 / 99$ 10:20:16 & & & \\
\hline$-1.000002 E-03$ & $4.721607 \mathrm{E}-03$ & & & & $3 / 17 / 99$ 10:20:19 & & & \\
\hline $9.999656 \mathrm{E}-04$ & $-4.723746 \mathrm{E}-03$ & & & & $3 / 17 / 99$ 10:20:22 & & & \\
\hline $9.999649 \mathrm{E}-04$ & $-4.723732 \mathrm{E}-03$ & & & & $3 / 17 / 99 \quad 10: 20: 24$ & & & \\
\hline$-1.000003 E-03$ & 4.721569E-03 & & & & $3 / 17 / 99$ 10:20:27 & & & \\
\hline$-1.000002 E-03$ & 4.721607E-03 & & & & $3 / 17 / 99$ 10:20:30 & & & \\
\hline $9.999658 \mathrm{E}-04$ & $-4.723721 E-03$ & & & & $3 / 17 / 99 \quad 10: 20: 33$ & & & \\
\hline $9.999656 \mathrm{E}-04$ & $-4.723724 \mathrm{E}-03$ & & & & $3 / 17 / 99 \quad 10: 20: 36$ & & & \\
\hline$-1.000002 E-03$ & $4.721635 \mathrm{E}-03$ & & & & $3 / 17 / 99 \quad 10: 20: 39$ & & & \\
\hline$-1.000002 \mathrm{E}-03$ & $4.721584 \mathrm{E}-03$ & & & & $3 / 17 / 99 \quad 10: 20: 41$ & & & \\
\hline $9.999656 \mathrm{E}-04$ & $-4.723736 \mathrm{E}-03$ & & & & $3 / 17 / 99 \quad 10: 20: 44$ & & & \\
\hline $9.999656 \mathrm{E}-04$ & $-4.723757 \mathrm{E}-03$ & & & & $3 / 17 / 99$ 10:20:47 & & & \\
\hline$-1.000002 E-03$ & $4.721641 \mathrm{E}-03$ & & & & $3 / 17 / 99 \quad 10: 20: 50$ & & & \\
\hline$-1.000002 E-03$ & 4.721591E-03 & & & & $3 / 17 / 99 \quad 10: 20: 53$ & & & \\
\hline 9.999655E-04 & $-4.723706 E-03$ & & & & $3 / 17 / 99 \quad 10: 20: 56$ & & & \\
\hline $9.999650 \mathrm{E}-04$ & $-4.723745 \mathrm{E}-03$ & & & & $3 / 17 / 99 \quad 10: 20: 58$ & & & \\
\hline$-1.000002 E-03$ & $4.721654 \mathrm{E}-03$ & & & & 3/17/99 10:21:01 & & & \\
\hline$-1.000002 E-03$ & $4.721619 E-03$ & & & & $3 / 17 / 99$ 10:21:04 & & & \\
\hline $9.999655 \mathrm{E}-04$ & $-4.723682 E-03$ & & & & $3 / 17 / 99$ 10:21:07 & & & \\
\hline $9.999838 \mathrm{E}-04$ & $4.722663 \mathrm{E}-03$ & 4.722740 & $1.512730 \mathrm{E}-05$ & 4.722573E-06 & $3 / 17 / 99$ 10:21:11 & 4975.491816 & 4.72726755 & \\
\hline & & & & & & & & \\
\hline
\end{tabular}


Tungsten.xls

\begin{tabular}{|c|c|c|c|c|c|c|c|c|}
\hline Current $(A)$ & Voltage (V) & Res.(ohms) & Std. Dev. Of $\Omega$ & Power (W) & Date/time & Cernox (ohms) & Cernox (K) 4th orde & er poly. \\
\hline Sample ID $=\mathrm{W}$ & Beam Energy $=p r$ & re-1.1 GeV & ne on target $=0$ & & & & & \\
\hline This is the cold date & a acquisition at 4.72 & $2 \mathrm{~K}$ prior to irradiatio & on at $1.1 \mathrm{GeV}$ & & & & & \\
\hline & & & & & & & & \\
\hline $2.000118 E-03$ & $-9.447686 \mathrm{E}-03$ & & & & $3 / 17 / 99$ 10:21:37 & & & \\
\hline$-2.000565 E-03$ & $9.446063 \mathrm{E}-03$ & & & & $3 / 17 / 99 \quad 10: 21: 40$ & & & \\
\hline$-2.000564 \mathrm{E}-03$ & $9.446070 \mathrm{E}-03$ & & & & $3 / 17 / 99$ 10:21:43 & & & \\
\hline $2.000127 \mathrm{E}-03$ & $-9.447685 \mathrm{E}-03$ & & - & & $3 / 17 / 99$ 10:21:45 & & & \\
\hline $2.000132 \mathrm{E}-03$ & $-9.447651 \mathrm{E}-03$ & & & & $3 / 17 / 99 \quad 10: 21: 48$ & & & \\
\hline$-2.000560 \mathrm{E}-03$ & $9.446028 \mathrm{E}-03$ & & & & $3 / 17 / 99$ 10:21:51 & & & \\
\hline$-2.000558 E-03$ & $9.446037 \mathrm{E}-03$ & & & & $3 / 17 / 99 \quad 10: 21: 54$ & & & \\
\hline $2.000121 \mathrm{E}-03$ & $-9.447750 \mathrm{E}-03$ & & & & $3 / 17 / 99$ 10:21:57 & & & \\
\hline $2.000129 \mathrm{E}-03$ & $-9.447660 \mathrm{E}-03$ & & & & $3 / 17 / 99 \quad 10: 22: 00$ & & & \\
\hline$-2000558 \mathrm{~F}-03$ & 9.446136E-03 & & & & $3 / 17 / 99 \quad 10: 22: 02$ & & & \\
\hline$-2.000559 \mathrm{E}-03$ & $9.446045 \mathrm{E}-03$ & & & & $3 / 17 / 9910: 22: 05$ & & & \\
\hline $2.000122 E-03$ & $-9.447613 \mathrm{E}-03$ & & & & $3 / 17 / 9910: 22: 08$ & & & \\
\hline $2.000120 \mathrm{E}-03$ & $-9.447670 \mathrm{E}-03$ & & & & $3 / 17 / 99 \quad 10: 22: 11$ & & & \\
\hline$-2.000563 E-03$ & $9.446087 \mathrm{E}-03$ & & & & $3 / 17 / 99$ 10:22:14 & & & \\
\hline$-2.000566 \mathrm{E}-03$ & $9.446148 E-03$ & & 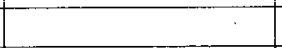 & & $3 / 17 / 99 \quad 10: 22: 17$ & & & \\
\hline $2.000121 \mathrm{E}-03$ & $-9.447677 E-03$ & & & & $3 / 17 / 99$ 10:22:19 & & & \\
\hline $2.000123 E-03$ & $-9.447690 \mathrm{E}-03$ & & & & $3 / 17 / 99$ 10:22:22 & & & \\
\hline$-2.000568 \mathrm{E}-03$ & $9.446122 E-03$ & & & & $3 / 17 / 99 \quad 10: 22: 25$ & & & \\
\hline$-2.000568 \mathrm{E}-03$ & $9.446100 \mathrm{E}-03$ & & & & $3 / 17 / 99 \quad 10: 22: 28$ & & & \\
\hline $2.000121 \mathrm{E}-03$ & $-9.447596 \mathrm{E}-03$ & & & & $3 / 17 / 9910: 22: 31$ & & & \\
\hline $2.000125 \mathrm{E}-03$ & $-9.447588 \mathrm{E}-03$ & & & & $3 / 17 / 99 \quad 10: 22: 34$ & & & \\
\hline$-2.000568 \mathrm{E}-03$ & $9.446275 \mathrm{E}-03$ & & & & $3 / 17 / 99 \quad 10: 22: 36$ & & & \\
\hline$-2.000573 E-03$ & $9.446157 \mathrm{E}-03$ & & & & $3 / 17 / 99 \quad 10: 22: 39$ & & & \\
\hline $2.000123 \mathrm{E}-03$ & $-9.447532 E-03$ & & & & $3 / 17 / 9910: 22: 42$ & & & \\
\hline $2.000121 \mathrm{E}-03$ & $-9.447506 \mathrm{E}-03$ & & & & $3 / 17 / 9910: 22: 45$ & & & \\
\hline$-2.000565 E-03$ & 9.446211E-03 & & & & $3 / 17 / 99$ 10:22:48 & & & \\
\hline$-2.000561 \mathrm{E}-03$ & $9.446262 \mathrm{E}-03$ & & & & $3 / 17 / 99 \quad 10: 22: 50$ & & & \\
\hline $2.000126 \mathrm{E}-03$ & $-9.447588 \mathrm{E}-03$ & & & & $3 / 17 / 99$ 10:22:53 & & & \\
\hline $2.000127 \mathrm{E}-03$ & $-9.447557 E-03$ & & & & $3 / 17 / 9910: 22: 56$ & & & \\
\hline$-2.000571 \mathrm{E}-03$ & $9.446160 \mathrm{E}-03$ & & & & $3 / 17 / 99 \quad 10: 22: 59$ & & & \\
\hline$-2.000563 E-03$ & $9.446180 \mathrm{E}-03$ & & & & $3 / 17 / 9910: 23: 02$ & & & \\
\hline $2.000130 \mathrm{E}-03$ & $-9.447687 E-03$ & & & & $3 / 17 / 9910: 23: 05$ & & & \\
\hline $2.000127 \mathrm{E}-03$ & $-9.447652 E-03$ & & & & $3 / 17 / 99$ 10:23:07 & & & \\
\hline$-2.000562 \mathrm{E}-03$ & $9.446053 \mathrm{E}-03$ & & & & $3 / 17 / 99$ 10:23:10 & & & \\
\hline$-2.000562 E-03$ & $9.446086 \mathrm{E}-03$ & & & & $3 / 17 / 99 \quad 10: 23: 13$ & & & \\
\hline $2.000126 \mathrm{E}-03$ & $-9.447586 \mathrm{E}-03$ & & & & $3 / 17 / 99$ 10:23:16 & & & \\
\hline $2.000127 \mathrm{E}-03$ & $-9.447574 E-03$ & & & & $3 / 17 / 99$ 10:23:19 & & & \\
\hline$-2.000559 E-03$ & $9.446095 \mathrm{E}-03$ & & & & $3 / 17 / 99$ 10:23:22 & & & \\
\hline$-2.000558 \mathrm{E}-03$ & 9.446166E-03 & & & & $3 / 17 / 99$ 10:23:24 & & & \\
\hline $2.000127 \mathrm{E}-03$ & $-9.447541 \mathrm{E}-03$ & & & & $3 / 17 / 99$ 10:23:27 & & & \\
\hline $2.000344 \mathrm{E}-03$ & $9.446874 \mathrm{E}-03$ & 4.722625 & 1.689062E-05 & $1.889694 \mathrm{E}-05$ & $3 / 17 / 99$ 10:23:31 & 4976.097765 & 4.726944835 & \\
\hline & & & & & & & & \\
\hline & & & & & & & & \\
\hline
\end{tabular}


Tungsten.xls

\begin{tabular}{|c|c|c|c|c|c|c|c|c|}
\hline \multirow{3}{*}{$\begin{array}{l}\text { Current }(A) \\
\text { Sample ID }=W \\
\text { Data at the end of } t\end{array}$} & Voltage (V) & Res.(ohms) & Std. Dev. Of $\Omega$ & \multirow{2}{*}{$\frac{\text { Power (W) }}{\text { ites }}$} & \multirow[t]{2}{*}{ Date/time } & \multirow[t]{2}{*}{ Cernox (ohms) } & \multicolumn{2}{|c|}{ Cernox $(\mathrm{K})$ 4th order poly. } \\
\hline & \multicolumn{2}{|c|}{ Beam Energy $=1.2 \mathrm{GeV}$} & on target $=60$ minutes & & & & & \\
\hline & the first irradiation a & at $1.2 \mathrm{GeV}$ & & & & & & \\
\hline & & & & & & & & \\
\hline $5.000211 \mathrm{E}-04$ & $-2.363556 \mathrm{E}-03$ & & & & $3 / 17 / 99$ 13:24:25 & & & \\
\hline$-5.000734 E-04$ & $2.362062 \mathrm{E}-03$ & & & & $3 / 17 / 99$ 13:24:28 & & & \\
\hline$-5.000729 E-04$ & $2.362064 \mathrm{E}-03$ & & & & $3 / 17 / 99$ 13:24:31 & & & \\
\hline $5.000210 \mathrm{E}-04$ & $-2.363556 \mathrm{E}-03$ & & & & 3/17/99 13:24:34 & & & \\
\hline $5.000220 \mathrm{E}-04$ & $-2.363552 \mathrm{E}-03$ & & & & $3 / 17 / 99$ 13:24:37 & & & \\
\hline$-5.000736 \mathrm{E}-04$ & $2.362088 \mathrm{E}-03$ & & & & 3/17/99 13:24:39 & & & \\
\hline$-5.000735 E-04$ & $2.362087 \mathrm{E}-03$ & & & & $3 / 17 / 99 \quad 13: 24: 42$ & & & \\
\hline $5.000221 E-04$ & $-2.363542 E-03$ & & & & $3 / 17 / 99$ 13:24:45 & & & \\
\hline $5.000219 \mathrm{E}-04$ & $-2.363522 \mathrm{E}-03$ & & & & $3 / 17 / 99 \quad 13: 24: 48$ & & & \\
\hline$-5.000734 \mathrm{E}-04$ & $2.362108 \mathrm{E}-03$ & & & & $3 / 17 / 99$ 13:24:51 & & & \\
\hline$-5.000735 E-04$ & $2.362108 \mathrm{E}-03$ & & & & $3 / 17 / 99 \quad 13: 24: 54$ & & & \\
\hline $5.000224 \mathrm{E}-04$ & $-2.363513 E-03$ & & & & $3 / 17 / 99$ 13:24:56 & & & \\
\hline $5.000219 \mathrm{E}-04$ & $-2.363498 \mathrm{E}-03$ & & & & $3 / 17 / 9913: 24: 59$ & & & \\
\hline$-5.000734 \mathrm{E}-04$ & $2.362115 \mathrm{E}-03$ & & & & $3 / 17 / 99$ 13:25:02 & & & \\
\hline$-5.000731 E-04$ & 2.362124E-03 & & & & $3 / 17 / 99$ 13:25:05 & & & \\
\hline $5.000221 E-04$ & $-2.363497 E-03$ & & & & $3 / 17 / 99$ 13:25:08 & & & \\
\hline $5.000219 E-04$ & $-2.363499 E-03$ & & & & 3/17/99 13:25:10 & & & \\
\hline$-5.000730 \mathrm{E}-04$ & $2.362115 \mathrm{E}-03$ & & & & $3 / 17 / 99$ 13:25:13 & & & \\
\hline$-5.000735 E-04$ & $2.362116 \mathrm{E}-03$ & & & & $3 / 17 / 9913: 25: 16$ & & & \\
\hline $5.000216 \mathrm{E}-04$ & $-2.363509 E-03$ & & & & $3 / 17 / 99$ 13:25:19 & & & \\
\hline $5.000217 \mathrm{E}-04$ & $-2.363495 E-03$ & & & & $3 / 17 / 99$ 13:25:22 & & & \\
\hline-5.000733 E-04 & $2.362131 \mathrm{E}-03$ & & & & $3 / 17 / 99 \quad 13: 25: 25$ & & & \\
\hline$-5.000726 \mathrm{E}-04$ & $2.362122 \mathrm{E}-03$ & & & & $3 / 17 / 99 \quad 13: 25: 27$ & & & \\
\hline $5.000224 \mathrm{E}-04$ & $-2.363501 \mathrm{E}-03$ & & & & $3 / 17 / 99$ 13:25:30 & & & \\
\hline $5.000224 \mathrm{E}-04$ & $-2.363507 \mathrm{E}-03$ & & & & $3 / 17 / 99$ 13:25:33 & & & \\
\hline$-5.000728 E-04$ & $2.362124 \mathrm{E}-03$ & & & & $3 / 17 / 99 \quad 13: 25: 36$ & & & \\
\hline$-5.000726 \mathrm{E}-04$ & $2.362128 \mathrm{E}-03$ & & & & $3 / 17 / 99$ 13:25:39 & & & \\
\hline $5.000224 \mathrm{E}-04$ & $-2.363500 \mathrm{E}-03$ & & & & $3 / 17 / 99$ 13:25:42 & & & \\
\hline $5.000227 \mathrm{E}-04$ & $-2.363505 E-03$ & & & & $3 / 17 / 99$ 13:25:44 & & & \\
\hline$-5.000732 \mathrm{E}-04$ & $2.362114 \mathrm{E}-03$ & & & & $3 / 17 / 99 \quad 13: 25: 47$ & & & \\
\hline$-5.000731 \mathrm{E}-04$ & $2.362116 \mathrm{E}-03$ & & & & $3 / 17 / 99$ 13:25:50 & & & \\
\hline $5.000226 \mathrm{E}-04$ & $-2.363514 \mathrm{E}-03$ & & & & $3 / 17 / 99$ 13:25:53 & & & \\
\hline $5.000226 \mathrm{E}-04$ & $-2.363511 E-03$ & & & & $3 / 17 / 99$ 13:25:56 & & & \\
\hline$-5.000735 \mathrm{E}-04$ & 2.362121E-03 & & & & $3 / 17 / 99$ 13:25:58 & & & \\
\hline$-5.000734 \mathrm{E}-04$ & $2.362114 \mathrm{E}-03$ & & & & 3/17/99 13:26:01 & & & \\
\hline $5.000226 \mathrm{E}-04$ & $-2.363524 \mathrm{E}-03$ & & & & $3 / 17 / 99$ 13:26:04 & & & \\
\hline 5.000227E-04 & $-2.363529 E-03$ & & & & $3 / 17 / 99$ 13:26:07 & & & \\
\hline$-5.000738 E-04$ & $2.362089 E-03$ & & & & $3 / 17 / 99$ 13:26:10 & & & \\
\hline$-5.000745 \mathrm{E}-04$ & $2.362111 \mathrm{E}-03$ & & & & $3 / 17 / 99$ 13:26:13 & & & \\
\hline $5.000220 \mathrm{E}-04$ & $-2.363523 E-03$ & & & & $3 / 17 / 9913: 26: 15$ & & & \\
\hline $5.000477 \mathrm{E}-04$ & $2.362813 \mathrm{E}-03$ & 4.725175 & $6.979432 \mathrm{E}-06$ & $1.181523 E-06$ & $3 / 17 / 99$ 13:26:20 & 4988.760772 & 4.720216954 & \\
\hline & & & & & & & & \\
\hline
\end{tabular}


Tungsten.xls

\begin{tabular}{|c|c|c|c|c|c|c|c|c|}
\hline \multirow{2}{*}{$\begin{array}{l}\text { Current }(A) \\
\text { Sample } I D=W\end{array}$} & Voltage (V) & Res.(ohms) & Std. Dev. Of $\Omega$ & \multirow{2}{*}{$\begin{array}{l}\text { Power }(\mathrm{W}) \\
\text { tes }\end{array}$} & \multirow[t]{2}{*}{ Date/time } & \multirow[t]{2}{*}{ Cernox (ohms) } & \multicolumn{2}{|c|}{ Cernox (K) 4th order poly. } \\
\hline & Beam Energy $=1.1$ & Time on & $n$ target $=60$ minut & & & & & \\
\hline \multicolumn{9}{|c|}{ Data at the end of the first irradiation at $1.1 \mathrm{GeV}$} \\
\hline & & & & & & & & \\
\hline $9.999664 \mathrm{E}-04$ & $-4.725873 E-03$ & & & & $3 / 17 / 99$ 13:27:14 & & & \\
\hline$-1.000001 E-03$ & 4.724303E-03 & & & & $3 / 17 / 99 \quad 13: 27: 17$ & & & \\
\hline$-1.000000 \mathrm{E}-03$ & 4.724303E-03 & & & & $3 / 17 / 99 \quad 13: 27: 20$ & & & \\
\hline $9.999662 \mathrm{E}-04$ & $-4.725895 \mathrm{E}-03$ & & & & $3 / 17 / 99$ 13:27:23 & & & \\
\hline 9.999657E-04 & $-4.725886 \mathrm{E}-03$ & & & & 3/17/99 13:27:26 & & & \\
\hline$-1.000001 \mathrm{E}-03$ & $4.724301 \mathrm{E}-03$ & & & & $3 / 17 / 99 \quad 13: 27: 29$ & & & \\
\hline$-1.000001 E-03$ & 4.724307E-03 & & & & 3/17/99 13:27:31 & & & \\
\hline $9.999665 \mathrm{E}-04$ & $-4.725897 \mathrm{E}-03$ & & & & $3 / 17 / 99 \quad 13: 27: 34$ & & & \\
\hline $9.999661 \mathrm{E}-04$ & $-4.725910 \mathrm{E}-03$ & & & & $3 / 17 / 99 \quad 13: 27: 37$ & & & \\
\hline$-1.000001 E-03$ & 4.724275E-03 & & & & $3 / 17 / 99$ 13:27:40 & & & \\
\hline$-1.000001 E-03$ & 4.724271E-03 & & & & $3 / 17 / 99$ 13:27:43 & & & \\
\hline $9.999664 \mathrm{E}-04$ & $-4.725942 E-03$ & & & & 3/17/99 13:27:46 & & & \\
\hline $9.999661 \mathrm{E}-04$ & $-4.725946 E-03$ & & & & $3 / 17 / 99 \quad 13: 27: 48$ & & & \\
\hline$-1.000001 E-03$ & 4.724259E-03 & & & & $3 / 17 / 99$ 13:27:51 & & & \\
\hline$-1.000000 \mathrm{E}-03$ & 4.724263E-03 & & & & $3 / 17 / 99$ 13:27:54 & & & \\
\hline $9.999669 \mathrm{E}-04$ & $-4.725939 \mathrm{E}-03$ & & & & $3 / 17 / 9913: 27: 57$ & & & \\
\hline $9.999663 \mathrm{E}-04$ & $-4.725950 E-03$ & & & & $3 / 17 / 99$ 13:28:00 & & & \\
\hline$-1.000001 E-03$ & $4.724258 \mathrm{E}-03$ & & & & 3/17/99 13:28:03 & & & \\
\hline$-1.000001 E-03$ & $4.724231 \mathrm{E}-03$ & & & & 3/17/99 13:28:05 & & & \\
\hline 9.999668E-04 & $-4.725978 E-03$ & & & & $3 / 17 / 9913: 28: 08$ & & & \\
\hline 9.999663E-04 & $-4.725968 \mathrm{E}-03$ & & & & 3/17/99 13:28:11 & & & \\
\hline$-1.000002 E-03$ & $4.724214 \mathrm{E}-03$ & & & & $3 / 17 / 99$ 13:28:14 & & & \\
\hline$-1.000001 \mathrm{E}-03$ & 4.724211E-03 & & & & $3 / 17 / 99$ 13:28:17 & & & \\
\hline $9.999663 \mathrm{E}-04$ & $-4.725985 \mathrm{E}-03$ & & & & 3/17/99 13:28:19 & & & \\
\hline $9.999662 \mathrm{E}-04$ & $-4.725989 \mathrm{E}-03$ & & & & $3 / 17 / 99$ 13:28:22 & & & \\
\hline$-1.000002 \mathrm{E}-03$ & 4.724213E-03 & & & & $3 / 17 / 99$ 13:28:25 & & & \\
\hline$-1.000001 E-03$ & 4.724189E-03 & & & & $3 / 17 / 99$ 13:28:28 & & & \\
\hline $9.999670 \mathrm{E}-04$ & $-4.726002 \mathrm{E}-03$ & & & & $3 / 17 / 99$ 13:28:31 & & & \\
\hline $9.999664 \mathrm{E}-04$ & $-4.726006 \mathrm{E}-03$ & & & & $3 / 17 / 99$ 13:28:34 & & & \\
\hline$-1.000002 \mathrm{E}-03$ & 4.724183E-03 & & & & $3 / 17 / 99$ 13:28:36 & & & \\
\hline$-1.000002 E-03$ & $4.724179 \mathrm{E}-03$ & & & & $3 / 17 / 99$ 13:28:39 & & & \\
\hline $9.999672 \mathrm{E}-04$ & $-4.726026 \mathrm{E}-03$ & & & & $3 / 17 / 99$ 13:28:42 & & & \\
\hline $9.999665 \mathrm{E}-04$ & $-4.726017 E-03$ & & & & $3 / 17 / 99$ 13:28:45 & & & \\
\hline$-1.000001 E-03$ & $4.724187 \mathrm{E}-03$ & & & & $3 / 17 / 99$ 13:28:48 & & & \\
\hline$-1.000001 E-03$ & $4.724168 \mathrm{E}-03$ & & & & $3 / 17 / 99$ 13:28:51 & & & \\
\hline $9.999672 E-04$ & $-4.726010 \mathrm{E}-03$ & & & & $3 / 17 / 99$ 13:28:53 & & & \\
\hline $9.999657 \mathrm{E}-04$ & $-4.726034 E-03$ & & & & $3 / 17 / 99$ 13:28:56 & & & \\
\hline$-1.000002 \mathrm{E}-03$ & $4.724181 \mathrm{E}-03$ & & & & $3 / 17 / 9913: 28: 59$ & & & \\
\hline$-1.000001 E-03$ & $4.724168 \mathrm{E}-03$ & & & & $3 / 17 / 99$ 13:29:02 & & & \\
\hline $9.999662 \mathrm{E}-04$ & $-4.726019 \mathrm{E}-03$ & & & & $3 / 17 / 99$ 13:29:05 & & & \\
\hline $9.999838 \mathrm{E}-04$ & $4.725098 \mathrm{E}-03$ & 4.725175 & $5.932730 \mathrm{E}-06$ & 4.725017E-06 & $3 / 17 / 99$ 13:29:09 & 4988.640685 & 4.720280612 & \\
\hline & & & & & & & & \\
\hline & & & & & & & & \\
\hline
\end{tabular}


Tungsten.xls

\begin{tabular}{|c|c|c|c|c|c|c|c|c|}
\hline \multirow{2}{*}{$\begin{array}{l}\text { Current (A) } \\
\text { Sample ID }=W\end{array}$} & Voltage $(\mathrm{V})$ & Res.(ohms) & & & \multirow[t]{2}{*}{ Date/time } & \multirow[t]{2}{*}{ Cernox (ohms) } & \multicolumn{2}{|c|}{ Cernox $(\mathrm{K})$ 4th order poly } \\
\hline & Beam Energy $=1.1$ & $.1 \mathrm{GeV} \quad$ Time on & $\begin{array}{c}\text { Std. Dev. Of } \Omega \quad \text { Power }(W) \\
\text { Time on target }=60 \text { minutes }\end{array}$ & & & & & \\
\hline \multicolumn{9}{|c|}{ Data at the end of the first irradiation at $1.1 \mathrm{GeV}$} \\
\hline & & & & & & & & \\
\hline $9.999656 \mathrm{E}-04$ & $-4.726015 \mathrm{E}-03$ & & & & $3 / 17 / 99$ 13:29:12 & & & \\
\hline$-1.000002 E-03$ & $4.724173 \mathrm{E}-03$ & & & & 3/17/99 13:29:15 & & & \\
\hline$-1.000002 E-03$ & $4.724198 \mathrm{E}-03$ & & & & 3/17/99 13:29:18 & & & \\
\hline $9.999662 \mathrm{E}-04$ & $-4.726017 \mathrm{E}-03$ & & & & $3 / 17 / 99$ 13:29:21 & & & \\
\hline $9.999656 \mathrm{E}-04$ & $-4.726020 E-03$ & & & & $3 / 17 / 99$ 13:29:23 & & & \\
\hline$-1.000002 E-03$ & $4.724164 \mathrm{E}-03$ & & & & 3/17/99 13:29:26 & & & \\
\hline$-1.000002 E-03$ & $4.724180 \mathrm{E}-03$ & & & & $3 / 17 / 99$ 13:29:29 & & & \\
\hline $9.999658 \mathrm{E}-04$ & $-4.726024 \mathrm{E}-03$ & & & & 3/17/99 13:29:32 & & & \\
\hline $9.999657 E-04$ & $-4.726013 E-03$ & & & & $3 / 17 / 99$ 13:29:35 & & & \\
\hline$-1.000003 E-03$ & 4.724191E-03 & & & & 3/17/99 13:29:38 & & & \\
\hline$-1.000002 E-03$ & $4.724156 \mathrm{E}-03$ & & & & $3 / 17 / 99$ 13:29:40 & & & \\
\hline $9.999663 E-04$ & $-4.726027 \mathrm{E}-03$ & & & & 3/17/99 13:29:43 & & & \\
\hline $9.999661 \mathrm{E}-04$ & $-4.726029 \mathrm{E}-03$ & & & & $3 / 17 / 99$ 13:29:46 & & & \\
\hline$-1.000003 E-03$ & 4.724177E-03 & & & & $3 / 17 / 9913: 29: 49$ & & & \\
\hline$-1.000002 \mathrm{E}-03$ & $4.724172 \mathrm{E}-03$ & & & & $3 / 17 / 99$ 13:29:52 & & & \\
\hline $9.999659 \mathrm{E}-04$ & $-4.726028 \mathrm{E}-03$ & & & & 3/17/99 13:29:55 & & & \\
\hline 9.999659E-04 & $-4.726032 E-03$ & & & & 3/17/99 13:29:57 & & & \\
\hline$-1.000002 E-03$ & $4.724170 \mathrm{E}-03$ & & & & $3 / 17 / 99$ 13:30:00 & & & \\
\hline$-1.000002 E-03$ & $4.724166 \mathrm{E}-03$ & & & & 3/17/99 13:30:03 & & & \\
\hline 9.999663E-04 & $-4.726046 \mathrm{E}-03$ & & & & 3/17/99 13:30:06 & & & \\
\hline $9.999662 E-04$ & $-4.726033 E-03$ & & & & $3 / 17 / 99$ 13:30:09 & & & \\
\hline$-1.000002 E-03$ & $4.724139 \mathrm{E}-03$ & & & & $3 / 17 / 99$ 13:30:12 & & & \\
\hline$-1.000002 \mathrm{E}-03$ & $4.724145 \mathrm{E}-03$ & & & & 3/17/99 13:30:14 & & & \\
\hline $9.999665 \mathrm{E}-04$ & $-4.726048 \mathrm{E}-03$ & & & & 3/17/99 13:30:17 & & & \\
\hline $9.999661 \mathrm{E}-04$ & $-4.726065 \mathrm{E}-03$ & & & & $3 / 17 / 99$ 13:30:20 & & & \\
\hline$-1.000003 E-03$ & $4.724141 \mathrm{E}-03$ & & & & $3 / 47 / 99$ 13:30:23 & & & \\
\hline$-1.000002 E-03$ & $4.724123 \mathrm{E}-03$ & & & & 3/17/99 13:30:26 & & & \\
\hline $9.999666 \mathrm{E}-04$ & $-4.726085 E-03$ & & & & $3 / 17 / 99$ 13:30:28 & & & \\
\hline $9.999662 \mathrm{E}-04$ & $-4.726084 \mathrm{E}-03$ & & & & $3 / 17 / 99$ 13:30:31 & & & \\
\hline$-1.000002 \mathrm{E}-03$ & $4.724112 \mathrm{E}-03$ & & & & $3 / 17 / 99$ 13:30:34 & & & \\
\hline$-1.000002 \mathrm{E}-03$ & 4.724109E-03 & & & & $3 / 17 / 99$ 13:30:37 & & & \\
\hline 9.999665E-04 & $-4.726098 \mathrm{E}-03$ & & & & $3 / 17 / 99$ 13:30:40 & & & \\
\hline $9.999658 \mathrm{E}-04$ & $-4.726101 \mathrm{E}-03$ & & & & $3 / 17 / 99$ 13:30:43 & & & \\
\hline$-1.000002 E-03$ & $4.724117 \mathrm{E}-03$ & & & & $3 / 17 / 99$ 13:30:45 & & & \\
\hline$-1.000002 E-03$ & $4.724094 \mathrm{E}-03$ & & & & $3 / 17 / 99$ 13:30:48 & & & \\
\hline $9.999665 \mathrm{E}-04$ & $-4.726101 \mathrm{E}-03$ & & & & $3 / 17 / 99$ 13:30:51 & & & \\
\hline $9.999662 \mathrm{E}-04$ & $-4.726106 \mathrm{E}-03$ & & & & $3 / 17 / 99$ 13:30:54 & & & \\
\hline$-1.000002 E-03$ & $4.724098 \mathrm{E}-03$ & & & & $3 / 17 / 99$ 13:30:57 & & & \\
\hline$-1.000001 E-03$ & $4.724080 \mathrm{E}-03$ & & & & $3 / 17 / 99$ 13:31:00 & & & \\
\hline $9.999665 \mathrm{E}-04$ & $-4.726111 \mathrm{E}-03$ & & & & $3 / 17 / 99$ 13:31:02 & & & \\
\hline $9.999841 \mathrm{E}-04$ & $4.725100 \mathrm{E}-03$ & 4.725175 & $5.783772 \mathrm{E}-06$ & $4.725019 \mathrm{E}-06$ & $3 / 17 / 99$ 13:31:07 & 4987.828427 & 4.720711262 & \\
\hline & & & & & & & & \\
\hline & & & & & & & & \\
\hline
\end{tabular}


Tungsten.xis

\begin{tabular}{|c|c|c|c|c|c|c|c|c|}
\hline \multirow{2}{*}{$\begin{array}{l}\text { Current }(A) \\
\text { Sample ID=W }\end{array}$} & Voltage (V) & Res.(ohms) & Std. Dev. Of $\Omega$ & \multirow{2}{*}{ Power (W) } & \multirow[t]{2}{*}{ Date/time } & \multirow[t]{2}{*}{ Cernox (ohms) } & \multicolumn{2}{|c|}{ Cernox $(K)$ 4th order poly. } \\
\hline & Beam Energy $=1$. & $1 \mathrm{GeV} \quad$ Time or & $n$ target $=60$ minut & & & & & \\
\hline \multicolumn{9}{|c|}{ Data at the end of the first irradiation at $1.1 \mathrm{GeV}$} \\
\hline & & & & & & & & \\
\hline $2.000127 \mathrm{E}-03$ & $-9.452584 E-03$ & & & & $3 / 17 / 99 \quad 13: 32: 15$ & & & \\
\hline$-2.000564 \mathrm{E}-03$ & $9.450922 E-03$ & & & & $3 / 17 / 99$ 13:32:17 & & & \\
\hline$-2.000558 E-03$ & $9.450897 E-03$ & & & & $3 / 17 / 9913: 32: 20$ & & & \\
\hline $2.000139 \mathrm{E}-03$ & $-9.452682 E-03$ & & & & $3 / 17 / 99$ 13:32:23 & & & \\
\hline $2.000133 \mathrm{E}-03$ & $-9.452602 \mathrm{E}-03$ & & & & $3 / 17 / 9913: 32: 26$ & & & \\
\hline$-2.000565 E-03$ & $9.450866 \mathrm{E}-03$ & & & & $3 / 17 / 99$ 13:32:29 & & & \\
\hline$-2.000562 E-03$ & $9.450843 E-03$ & & & & $3 / 17 / 9913: 32: 32$ & & & \\
\hline $2.000137 \mathrm{E}-03$ & $-9.452632 E-03$ & & & & 3/17/99 13:32:34 & & & \\
\hline $2.000128 \mathrm{E}-03$ & $-9.452620 \mathrm{E}-03$ & & & & $3 / 17 / 99$ 13:32:37 & & & \\
\hline$-2.000571 \mathrm{E}-03$ & $9.450948 \mathrm{E}-03$ & & & & $3 / 17 / 99$ 13:32:40 & & & \\
\hline$-2.000569 \mathrm{E}-03$ & $9.450981 \mathrm{E}-03$ & & & & $3 / 17 / 9913: 32: 43$ & & & \\
\hline $2.000131 E-03$ & $-9.452518 E-03$ & & & & $3 / 17 / 99$ 13:32:46 & & & \\
\hline $2.000134 \mathrm{E}-03$ & $-9.452538 \mathrm{E}-03$ & & & & $3 / 17 / 99$ 13:32:49 & & & \\
\hline$-2.000564 \mathrm{E}-03$ & $9.450936 \mathrm{E}-03$ & & & & $3 / 17 / 99$ 13:32:51 & & & \\
\hline$-2.000566 \mathrm{E}-03$ & $9.450892 \mathrm{E}-03$ & & & & $3 / 17 / 99$ 13:32:54 & & & \\
\hline $2.000133 \mathrm{E}-03$ & $-9.452686 \mathrm{E}-03$ & & & & $3 / 17 / 99$ 13:32:57 & & & \\
\hline $2.000137 \mathrm{E}-03$ & $-9.452686 E-03$ & & & & 3/17/99 13:33:00 & & & \\
\hline$-2.000561 E-03$ & $9.450932 \mathrm{E}-03$ & & & & 3/17/99 13:33:03 & & & \\
\hline$-2.000561 E-03$ & $9.450957 \mathrm{E}-03$ & & & & $3 / 17 / 99$ 13:33:06 & & & \\
\hline $2.000136 \mathrm{E}-03$ & $-9.452498 E-03$ & & & & $3 / 17 / 99$ 13:33:08 & & & \\
\hline $2.000135 \mathrm{E}-03$ & $-9.452506 \mathrm{E}-03$ & & & & $3 / 17 / 99$ 13:33:11 & & & \\
\hline$-2.000563 E-03$ & $9.450993 \mathrm{E}-03$ & & & & $3 / 17 / 99$ 13:33:14 & & & \\
\hline$-2.000560 \mathrm{E}-03$ & $9.450981 \mathrm{E}-03$ & & & & $3 / 17 / 99$ 13:33:17 & & & \\
\hline $2.000133 E-03$ & $-9.452524 E-03$ & & & & $3 / 17 / 9913: 33: 20$ & & & \\
\hline $2.000129 E-03$ & $-9.452551 \mathrm{E}-03$ & & & & $3 / 17 / 9913: 33: 22$ & & & \\
\hline$-2.000561 E-03$ & $9.450944 \mathrm{E}-03$ & & & & $3 / 17 / 9913: 33: 25$ & & & \\
\hline$-2.000555 \mathrm{E}-03$ & $9.450911 \mathrm{E}-03$ & & & & $3 / 17 / 99$ 13:33:28 & & & \\
\hline $2.000130 \mathrm{E}-03$ & $-9.452637 \mathrm{E}-03$ & & & & 3/17/99 13:33:31 & & & \\
\hline $2.000134 \mathrm{E}-03$ & $-9.452657 E-03$ & & & & $3 / 17 / 99$ 13:33:34 & & & \\
\hline$-2.000559 \mathrm{E}-03$ & $9.450943 \mathrm{E}-03$ & & & & $3 / 17 / 99$ 13:33:37 & & & \\
\hline$-2.000557 \mathrm{E}-03$ & $9.450926 \mathrm{E}-03$ & & & & $3 / 17 / 99$ 13:33:39 & & & \\
\hline $2.000140 \mathrm{E}-03$ & $-9.452678 E-03$ & & & & $3 / 17 / 99$ 13:33:42 & & & \\
\hline $2.000137 \mathrm{E}-03$ & $-9.452723 \mathrm{E}-03$ & & & & $3 / 17 / 99$ 13:33:45 & & & \\
\hline$-2.000560 \mathrm{E}-03$ & $9.450845 E-03$ & & & & $3 / 17 / 99$ 13:33:48 & & & \\
\hline$-2.000554 \mathrm{E}-03$ & $9.450866 \mathrm{E}-03$ & & & & $3 / 17 / 99$ 13:33:51 & & & \\
\hline $2.000140 \mathrm{E}-03$ & $-9.452622 \mathrm{E}-03$ & & & & $3 / 17 / 9913: 33: 54$ & & & \\
\hline $2.000139 E-03$ & $-9.452598 \mathrm{E}-03$ & & & & $3 / 17 / 99$ 13:33:56 & & & \\
\hline$-2.000559 \mathrm{E}-03$ & $9.450917 \mathrm{E}-03$ & & & & $3 / 17 / 99$ 13:33:59 & & & \\
\hline$-2.000558 E-03$ & $9.450911 \mathrm{E}-03$ & & & & $3 / 17 / 99$ 13:34:02 & & & \\
\hline $2.000143 E-03$ & $-9.452626 \mathrm{E}-03$ & & & & $3 / 17 / 9913: 34: 05$ & & & \\
\hline $2.000348 E-03$ & $9.451764 \mathrm{E}-03$ & 4.725060 & $1.277009 \mathrm{E}-05$ & $1.890685 \mathrm{E}-05$ & $3 / 17 / 9913: 34: 09$ & 4986.978958 & 4.721161775 & \\
\hline & & & & & & & & \\
\hline & & & & & & & & \\
\hline
\end{tabular}


Tungsten.xls

\begin{tabular}{|c|c|c|c|c|c|c|c|c|}
\hline \multirow{3}{*}{\begin{tabular}{|l|} 
Current $(A)$ \\
Sample ID $=W$ \\
Fnd of second and
\end{tabular}} & Voltage $(\mathrm{V})$ & Res.(ohms) & & & \multirow[t]{2}{*}{ Date/time } & \multirow[t]{2}{*}{ Cernox (ohms) } & \multicolumn{2}{|c|}{ Cernox $(K) 4$ th order poly. } \\
\hline & Beam Energy $=1$ & $1.1 \mathrm{GeV}$ & \multicolumn{2}{|c|}{$\begin{array}{l}\text { i) } \quad \text { Std. Dev. Of } \Omega \text { Power }(W) \\
\text { Time on target }=135 \text { minutes }\end{array}$} & & & & \\
\hline & \multicolumn{8}{|c|}{ End of second and last irradiation at $1.1 \mathrm{GeV}$} \\
\hline & & & & & & & & \\
\hline $5.000220 E-04$ & $-2.367571 E-03$ & & & & $3 / 17 / 99$ 15:45:02 & & & \\
\hline$-5.000575 E-04$ & $2.365617 \mathrm{E}-03$ & & & & $3 / 17 / 9915: 45: 05$ & & & \\
\hline$-5.000571 E-04$ & $2.365634 \mathrm{E}-03$ & & & & $3 / 17 / 99$ 15:45:08 & & & \\
\hline $5.000225 E-04$ & $-2.367583 \mathrm{E}-03$ & & & & $3 / 17 / 99$ 15:45:10 & & & \\
\hline $5.000225 E-04$ & $-2.367588 E-03$ & & & & $3 / 17 / 99$ 15:45:13 & & & \\
\hline$-5.000581 E-04$ & $2.365631 \mathrm{E}-03$ & & & & $3 / 17 / 99$ 15:45:16 & & & \\
\hline$-5.000580 E-04$ & $2.365630 \mathrm{E}-03$ & & & & $3 / 17 / 9915: 45: 19$ & & & \\
\hline $5.000225 E-04$ & $-2.367578 \mathrm{E}-03$ & & & & $3 / 17 / 99 \quad 15: 45: 22$ & & & \\
\hline $5.000228 E-04$ & $-2.367581 E-03$ & & & & $3 / 17 / 99 \quad 15: 45: 25$ & & & \\
\hline$-5.000578 \mathrm{E}-04$ & $2.365624 \mathrm{E}-03$ & & & & $3 / 17 / 9915: 45: 27$ & & & \\
\hline$-5.000572 \mathrm{E}-04$ & $2.365637 \mathrm{E}-03$ & & & & $3 / 17 / 9915: 45: 30$ & & & \\
\hline $5.000228 \mathrm{E}-04$ & $-2.367568 \mathrm{E}-03$ & & & & $3 / 17 / 99$ 15:45:33 & & & \\
\hline $5.000230 \mathrm{E}-04$ & $-2.367556 \mathrm{E}-03$ & & & & $3 / 17 / 99 \quad 15: 45: 36$ & & & \\
\hline$-5.000576 \mathrm{E}-04$ & $2.365633 \mathrm{E}-03$ & & & & $3 / 17 / 9915: 45: 39$ & & & \\
\hline$-5.000580 E-04$ & $2.365655 \mathrm{E}-03$ & & & & $3 / 17 / 9915: 45: 41$ & & & \\
\hline $5.000227 \mathrm{E}-04$ & $-2.367546 \mathrm{E}-03$ & & & & $3 / 17 / 99$ 15:45:44 & & & \\
\hline 5.000231E-04 & $-2.367557 \mathrm{E}-03$ & & & & $3 / 17 / 99$ 15:45:47 & & & \\
\hline$-5.000584 \mathrm{E}-04$ & $2.365649 E-03$ & & & & $3 / 17 / 99$ 15:45:50 & & & \\
\hline$-5.000583 E-04$ & $2.365652 E-03$ & & & & $3 / 17 / 99$ 15:45:53 & & & \\
\hline 5.000227E-04 & $-2.367550 \mathrm{E}-03$ & & & & $3 / 17 / 99$ 15:45:56 & & & \\
\hline $5.000228 \mathrm{E}-04$ & $-2.367558 E-03$ & & & & $3 / 17 / 99$ 15:45:58 & & & \\
\hline$-5.000579 \mathrm{E}-04$ & $2.365646 \mathrm{E}-03$ & & & & $3 / 17 / 99$ 15:46:01 & & & \\
\hline$-5.000575 E-04$ & $2.365663 \mathrm{E}-03$ & & & & $3 / 17 / 99$ 15:46:04 & & & \\
\hline $5.000231 E-04$ & $-2.367552 \mathrm{E}-03$ & & & & $3 / 17 / 99$ 15:46:07 & & & \\
\hline $5.000224 \mathrm{E}-04$ & $-2.367550 \mathrm{E}-03$ & & & & $3 / 17 / 99$ 15:46:10 & & & \\
\hline$-5.000581 \mathrm{E}-04$ & $2.365658 \mathrm{E}-03$ & & & & $3 / 17 / 99 \quad 15: 46: 13$ & & & \\
\hline$-5.000581 E-04$ & $2.365652 E-03$ & & & & $3 / 17 / 9915: 46: 15$ & & & \\
\hline $5.000228 \mathrm{E}-04$ & $-2.367551 \mathrm{E}-03$ & & & & $3 / 17 / 99$ 15:46:18 & & & \\
\hline $5.000231 \mathrm{E}-04$ & $-2.367547 \mathrm{E}-03$ & & & & $3 / 17 / 99$ 15:46:21 & & & \\
\hline$-5.000581 \mathrm{E}-04$ & $2.365656 \mathrm{E}-03$ & & & & $3 / 17 / 9915: 46: 24$ & & & \\
\hline$-5.000579 \mathrm{E}-04$ & $2.365660 \mathrm{E}-03$ & & & & $3 / 17 / 9915: 46: 27$ & & & \\
\hline $5.000226 \mathrm{E}-04$ & $-2.367545 \mathrm{E}-03$ & & & & $3 / 17 / 9915: 46: 30$ & & & \\
\hline 5.000227E-04 & $-2.367557 E-03$ & & & & $3 / 17 / 99 \quad 15: 46: 32$ & & & \\
\hline$-5.000582 E-04$ & $2.365645 \mathrm{E}-03$ & & & & $3 / 17 / 99$ 15:46:35 & & & \\
\hline$-5.000580 \mathrm{E}-04$ & $2.365648 \mathrm{E}-03$ & & & & $3 / 17 / 99$ 15:46:38 & & & \\
\hline $5.000228 \mathrm{E}-04$ & $-2.367541 E-03$ & & & & $3 / 17 / 99$ 15:46:41 & & & \\
\hline $5.000228 \mathrm{E}-04$ & $-2.367552 \mathrm{E}-03$ & & & & $3 / 17 / 9915: 46: 44$ & & & \\
\hline$-5.000583 E-04$ & 2.365657E-03 & & & & $3 / 17 / 99$ 15:46:47 & & & \\
\hline$-5.000578 \mathrm{E}-04$ & $2.365657 \mathrm{E}-03$ & & & & $3 / 17 / 99$ 15:46:49 & & & \\
\hline $5.000236 \mathrm{E}-04$ & $-2.367540 \mathrm{E}-03$ & & & & $3 / 17 / 99$ 15:46:52 & & & \\
\hline $5.000403 \mathrm{E}-04$ & $2.366602 \mathrm{E}-03$ & 4.732822 & 8.479429E-06 & 1.183396E-06 & $3 / 17 / 99$ 15:46:56 & 4976.458532 & 4.726752732 & \\
\hline & & & & & & & & \\
\hline & & & & & & & & \\
\hline
\end{tabular}


Tungsten.xls

\begin{tabular}{|c|c|c|c|c|c|c|c|c|}
\hline Current $(A)$ & Voltage $(\mathrm{V})$ & Res.(ohms) & Std. Dev. Of $\Omega$ & Power (W) & Date/time & Cernox (ohms) & Cernox (K) 4th orde & er poly. \\
\hline Sample ID $=W$ & Beam Energy $=1$ & $1.1 \mathrm{GeV}$ & $n$ target $=135$ minut & & & & & \\
\hline End of second and & last irradiation at 1 & $1.1 \mathrm{GeV}$ & & & & & & \\
\hline & & & & & & & & \\
\hline $9.999712 \mathrm{E}-04$ & $-4.733664 \mathrm{E}-03$ & & & & $3 / 17 / 99$ 15:47:34 & & & \\
\hline$-9.999903 \mathrm{E}-04$ & $4.731771 \mathrm{E}-03$ & & & & $3 / 17 / 99$ 15:47:37 & & & \\
\hline$-9.999899 E-04$ & $4.731776 \mathrm{E}-03$ & & & & $3 / 17 / 99$ 15:47:40 & & & \\
\hline $9.999721 \mathrm{E}-04$ & $-4.733666 \mathrm{E}-03$ & & & & $3 / 17 / 9915: 47: 42$ & & & \\
\hline $9.999714 \mathrm{E}-04$ & $-4.733669 \mathrm{E}-03$ & & & & $3 / 17 / 99 \quad 15: 47: 45$ & & & \\
\hline$-9.999914 E-04$ & $4.731781 \mathrm{E}-03$ & & & & $3 / 17 / 9915: 47: 48$ & & & \\
\hline$-9.999910 E-04$ & 4.731773E-03 & & & & $3 / 17 / 9915: 47: 51$ & & & \\
\hline 9.999714E-04 & $-4.733674 \mathrm{E}-03$ & & & & $3 / 17 / 99$ 15:47:54 & & & \\
\hline $9.999715 \mathrm{E}-04$ & $-4.733684 E-03$ & & & & $3 / 17 / 9915: 47: 57$ & & & \\
\hline$-9.999913 E-04$ & $4.731759 \mathrm{E}-03$ & & & & $3 / 17 / 99$ 15:47:59 & & & \\
\hline$-9.999906 E-04$ & $4.731775 \mathrm{E}-03$ & & & & $3 / 17 / 99$ 15:48:02 & & & \\
\hline $9.999718 \mathrm{E}-04$ & $-4.733672 \mathrm{E}-03$ & & & & $3 / 17 / 9915: 48: 05$ & & & \\
\hline $9.999714 \mathrm{E}-04$ & $-4.733676 \mathrm{E}-03$ & & & & $3 / 17 / 9915: 48: 08$ & & & \\
\hline$-9.999914 \mathrm{E}-04$ & $4.731776 \mathrm{E}-03$ & & & & $3 / 17 / 99$ 15:48:11 & & & \\
\hline$-9.999910 E-04$ & 4.731781E-03 & & & & $3 / 17 / 99$ 15:48:14 & & & \\
\hline $9.999712 \mathrm{E}-04$ & $-4.733666 \mathrm{E}-03$ & & & & $3 / 17 / 99$ 15:48:16 & & & \\
\hline $9.999713 \mathrm{E}-04$ & $-4.733667 E-03$ & & & & $3 / 17 / 99$ 15:48:19 & & & \\
\hline$-9.999913 E-04$ & $4.731779 \mathrm{E}-03$ & & & & $3 / 17 / 9915: 48: 22$ & & & \\
\hline$-9.999909 E-04$ & $4.731786 \mathrm{E}-03$ & & & & $3 / 17 / 9915: 48: 25$ & & & \\
\hline $9.999715 \mathrm{E}-04$ & $-4.733655 E-03$ & & & & $3 / 17 / 99$ 15:48:28 & & & \\
\hline $9.999712 \mathrm{E}-04$ & $-4.733663 \mathrm{E}-03$ & & & & $3 / 17 / 99$ 15:48:30 & & & \\
\hline$-9.999920 \mathrm{E}-04$ & $4.731780 \mathrm{E}-03$ & & & & $3 / 17 / 99$ 15:48:33 & & & \\
\hline$-9.999914 E-04$ & $4.731785 \mathrm{E}-03$ & & & & $3 / 17 / 9915: 48: 36$ & & & \\
\hline $9.999721 \mathrm{E}-04$ & $-4.733662 \mathrm{E}-03$ & & & & $3 / 17 / 99$ 15:48:39 & & & \\
\hline $9.999718 E-04$ & $-4.733673 \mathrm{E}-03$ & & & & $3 / 17 / 99 \quad 15: 48: 42$ & & & \\
\hline$-9.999922 E-04$ & 4.731783E-03 & & & & $3 / 17 / 9915: 48: 45$ & & & \\
\hline$-9.999917 E-04$ & 4.731779E-03 & & & & $3 / 17 / 9915: 48: 47$ & & & \\
\hline $9.999714 \mathrm{E}-04$ & $-4.733659 \mathrm{E}-03$ & & & & $3 / 17 / 9915: 48: 50$ & & & \\
\hline $9.999707 \mathrm{E}-04$ & $-4.733666 \mathrm{E}-03$ & & & & $3 / 17 / 9915: 48: 53$ & & & \\
\hline$-9.999924 \mathrm{E}-04$ & $4.731762 \mathrm{E}-03$ & & & & $3 / 17 / 9915: 48: 56$ & & & \\
\hline$-9.999919 \mathrm{E}-04$ & $4.731770 \mathrm{E}-03$ & & & & $3 / 17 / 9915: 48: 59$ & & & \\
\hline $9.999711 \mathrm{E}-04$ & $-4.733662 \mathrm{E}-03$ & & & & $3 / 17 / 99$ 15:49:02 & & & \\
\hline $9.999706 \mathrm{E}-04$ & $-4.733677 \mathrm{E}-03$ & & & & $3 / 17 / 99$ 15:49:04 & & & \\
\hline$-9.999929 \mathrm{E}-04$ & $4.731786 \mathrm{E}-03$ & & & & $3 / 17 / 9915: 49: 07$ & & & \\
\hline$-9.999925 E-04$ & $4.731768 \mathrm{E}-03$ & & & & $3 / 17 / 99$ 15:49:10 & & & \\
\hline $9.999712 \mathrm{E}-04$ & $-4.733644 \mathrm{E}-03$ & & & & $3 / 17 / 99$ 15:49:13 & & & \\
\hline 9.999705E-04 & $-4.733656 \mathrm{E}-03$ & & & & $3 / 17 / 99$ 15:49:16 & & & \\
\hline$-9.999927 E-04$ & $4.731783 \mathrm{E}-03$ & & & & $3 / 17 / 99$ 15:49:19 & & & \\
\hline$-9.999922 E-04$ & 4.731783E-03 & & & & $3 / 17 / 9915: 49: 21$ & & & \\
\hline $9.999715 \mathrm{E}-04$ & $-4.733665 \mathrm{E}-03$ & & & & $3 / 17 / 99$ 15:49:24 & & & \\
\hline $9.999814 \mathrm{E}-04$ & $4.732721 \mathrm{E}-03$ & 4.732809 & $5.372182 \mathrm{E}-06$ & $4.732638 \mathrm{E}-06$ & $3 / 17 / 99$ 15:49:28 & 4977.072556 & 4.726425831 & \\
\hline & & & & & & & & \\
\hline & & & & & & & & \\
\hline
\end{tabular}


Tungsten.xls

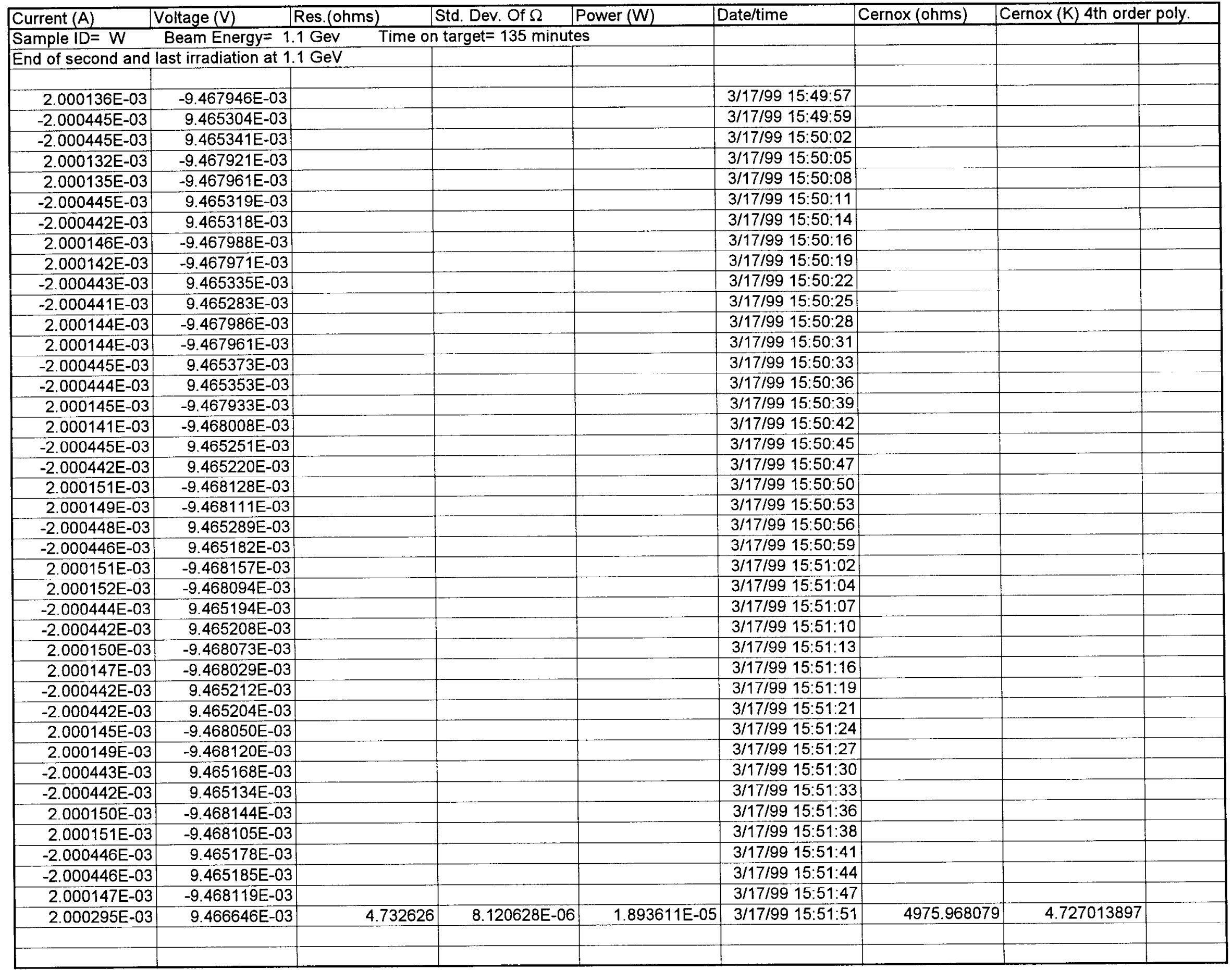


Tungsten.xls

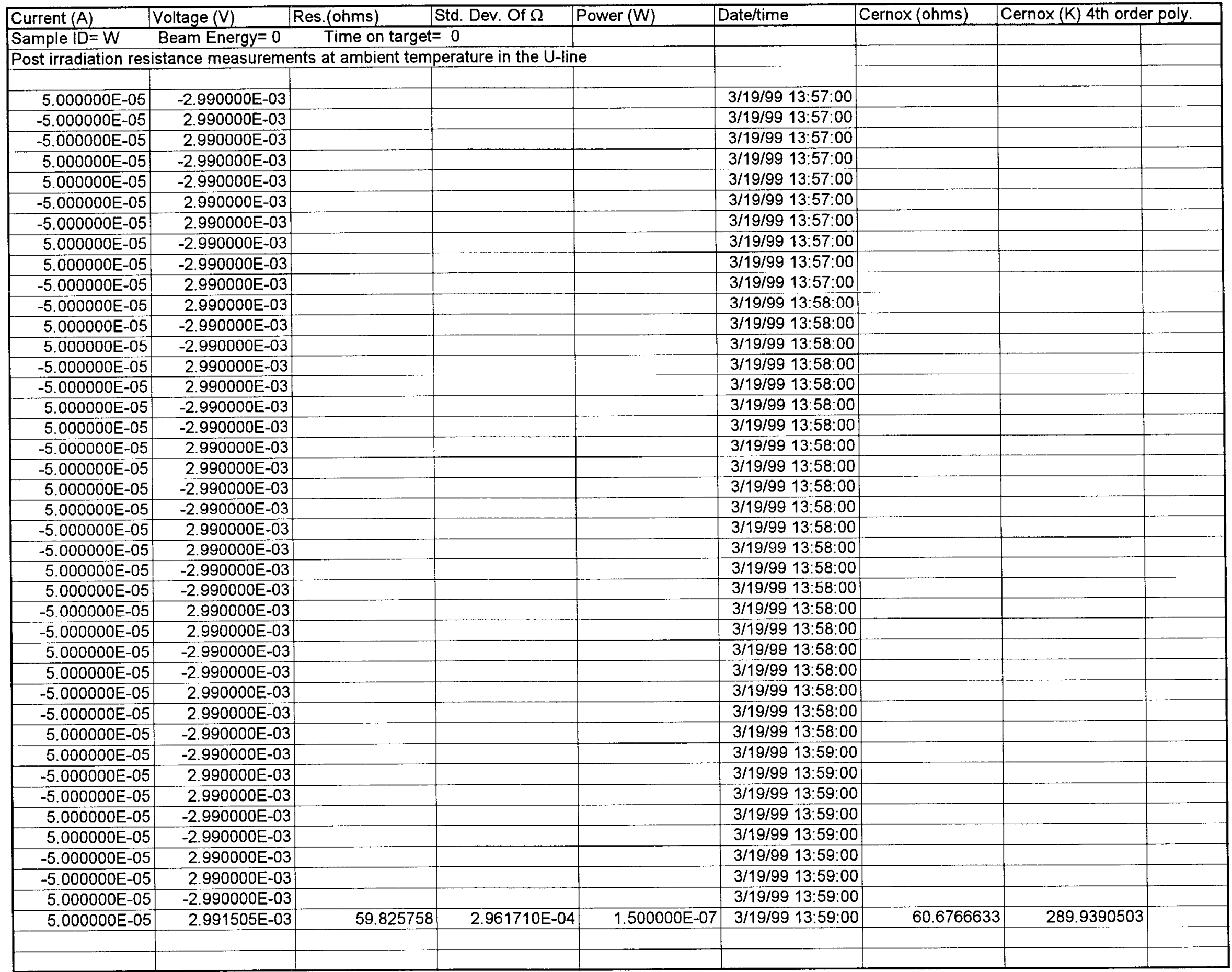


Tungsten.xls

\begin{tabular}{|c|c|c|c|c|c|c|c|c|}
\hline \multirow{2}{*}{$\begin{array}{l}\text { Current }(A) \\
\text { Sample ID=W }\end{array}$} & Voltage (V) & Res.(ohms) & \multirow{2}{*}{$\begin{array}{l}\text { Std. Dev. Of } \Omega \\
t=0\end{array}$} & \multirow[t]{2}{*}{ Power (W) } & \multirow[t]{2}{*}{ Date/time } & \multirow[t]{2}{*}{ Cernox (ohms) } & \multicolumn{2}{|c|}{ Cernox $(K)$ 4th order poly. } \\
\hline & Beam Energy $=0$ & Time on target- & & & & & & \\
\hline \multicolumn{9}{|c|}{ Post irradiation resistance measurements at ambient temperature in the U-line } \\
\hline & & & & & & & & \\
\hline $5.000000 \mathrm{E}-04$ & $-2.990000 \mathrm{E}-02$ & & & & 3/19/99 13:59:00 & & & \\
\hline$-5.000000 E-04$ & $2.990000 \mathrm{E}-02$ & & & & $3 / 19 / 99$ 13:59:00 & & & \\
\hline$-5.000000 \mathrm{E}-04$ & $2.990000 \mathrm{E}-02$ & & & & 3/19/99 13:59:00 & & & \\
\hline $5.000000 \mathrm{E}-04$ & $-2.990000 \mathrm{E}-02$ & & & & 3/19/99 13:59:00 & & & \\
\hline $5.000000 \mathrm{E}-04$ & $-2.990000 E-02$ & & & & 3/19/99 13:59:00 & & & \\
\hline$-5.000000 \mathrm{E}-04$ & $2.990000 \mathrm{E}-02$ & & & & $3 / 19 / 99$ 13:59:00 & & & \\
\hline$-5.000000 E-04$ & $2.990000 \mathrm{E}-02$ & & & & $3 / 19 / 99$ 13:59:00 & & & \\
\hline $5.000000 E-04$ & $-2.990000 \mathrm{E}-02$ & & & & 3/19/99 13:59:00 & & & \\
\hline 5.000000 E-04 & $-2.990000 \mathrm{E}-02$ & & & & $3 / 19 / 99$ 13:59:00 & & & \\
\hline$-5.000000 \mathrm{E}-04$ & $2.990000 \mathrm{E}-02$ & & & & $3 / 19 / 9914: 00: 00$ & & & \\
\hline$-5.000000 E-04$ & $2.990000 \mathrm{E}-02$ & & & & $3 / 19 / 9914: 00: 00$ & & & \\
\hline $5.000000 \mathrm{E}-04$ & $-2.990000 \mathrm{E}-02$ & & & & $3 / 19 / 9914: 00: 00$ & & & \\
\hline $5.000000 \mathrm{E}-04$ & $-2.990000 \mathrm{E}-02$ & & & & $3 / 19 / 9914: 00: 00$ & & & \\
\hline$-5.000000 E-04$ & $2.990000 \mathrm{E}-02$ & & & & $3 / 19 / 9914: 00: 00$ & & & \\
\hline$-5.000000 \mathrm{E}-04$ & $2.990000 \mathrm{E}-02$ & & & & $3 / 19 / 9914: 00: 00$ & & & \\
\hline $5.000000 \mathrm{E}-04$ & $-2.990000 \mathrm{E}-02$ & & & & $3 / 19 / 9914: 00: 00$ & & & \\
\hline $5.000000 \mathrm{E}-04$ & $-2.990000 \mathrm{E}-02$ & & & & 3/19/99 14:00:00 & & & \\
\hline$-5.000000 E-04$ & $2990000 \mathrm{E}-02$ & & & & $3 / 19 / 9914: 00: 00$ & & & \\
\hline$-5.000000 E-04$ & $2.990000 \mathrm{E}-02$ & & & & $3 / 19 / 99$ 14:00:00 & & & \\
\hline 5.000000 E-04 & $-2.990000 \mathrm{E}-02$ & & & & 3/19/99 14:00:00 & & & \\
\hline $5.000000 E-04$ & $-2.990000 \mathrm{E}-02$ & & & & $3 / 19 / 9914: 00: 00$ & & & \\
\hline$-5.000000 E-04$ & $2.990000 \mathrm{E}-02$ & & & & $3 / 19 / 9914: 00: 00$ & & & \\
\hline$-5.000000 E-04$ & $2.990000 E-02$ & & & & $3 / 19 / 9914: 00: 00$ & & & \\
\hline $5.000000 \mathrm{E}-04$ & $-2.990000 \mathrm{E}-02$ & & & & $3 / 19 / 99$ 14:00:00 & & & \\
\hline $5.000000 \mathrm{E}-04$ & $-2.990000 \mathrm{E}-02$ & & & & $3 / 19 / 9914: 00: 00$ & & & \\
\hline$-5.000000 E-04$ & $2.990000 \mathrm{E}-02$ & & & & $3 / 19 / 9914: 00: 00$ & & & \\
\hline$-5.000000 E-04$ & $2.990000 \mathrm{E}-02$ & & & & $3 / 19 / 9914: 00: 00$ & & & \\
\hline $5.000000 \mathrm{E}-04$ & $-2.990000 E-02$ & & & & $3 / 19 / 9914: 00: 00$ & & & \\
\hline $5.000000 \mathrm{E}-04$ & $-2.990000 \mathrm{E}-02$ & & & & $3 / 19 / 9914: 00: 00$ & & & \\
\hline$-5.000000 E-04$ & $2.990000 \mathrm{E}-02$ & & & & $3 / 19 / 99$ 14:00:00 & & & \\
\hline$-5.000000 \mathrm{E}-04$ & $2.990000 \mathrm{E}-02$ & & & & $3 / 19 / 9914: 01: 00$ & & & \\
\hline $5.000000 \mathrm{E}-04$ & $-2.990000 \mathrm{E}-02$ & & & & $3 / 19 / 99$ 14:01:00 & & & \\
\hline $5.000000 \mathrm{E}-04$ & $-2.990000 \mathrm{E}-02$ & & & & $3 / 19 / 99$ 14:01:00 & & & \\
\hline$-5.000000 \mathrm{E}-04$ & $2.990000 \mathrm{E}-02$ & & & & $3 / 19 / 99$ 14:01:00 & & & \\
\hline$-5.000000 \mathrm{E}-04$ & $2.990000 E-02$ & & & & $3 / 19 / 99$ 14:01:00 & & & \\
\hline $5.000000 \mathrm{E}-04$ & $-2.990000 \mathrm{E}-02$ & & & & $3 / 19 / 99$ 14:01:00 & & & \\
\hline $5.000000 \mathrm{E}-04$ & $-2.990000 \mathrm{E}-02$ & & & & 3/19/99 14:01:00 & & & \\
\hline$-5.000000 \mathrm{E}-04$ & $2.990000 \mathrm{E}-02$ & & & & $3 / 19 / 9914: 01: 00$ & & & \\
\hline$-5.000000 E-04$ & $2.990000 \mathrm{E}-02$ & & & & $3 / 19 / 9914: 01: 00$ & & & \\
\hline $5.000000 \mathrm{E}-04$ & $-2.990000 \mathrm{E}-02$ & & & & $3 / 19 / 99$ 14:01:00 & & & \\
\hline 5.000000 E-04 & $2.991023 \mathrm{E}-02$ & 59.815396 & $2.125810 \mathrm{E}-04$ & $1.500000 \mathrm{E}-05$ & $3 / 19 / 9914: 01: 00$ & 60.67032044 & 289.9741721 & \\
\hline
\end{tabular}


TUNGSTEN.XIS

\begin{tabular}{|c|c|c|c|c|c|c|c|c|}
\hline Current (A) & Voltage (V) & Res.(ohms) & Std. Dev. Of $\Omega$ & Power (W) & Date/time & Cernox (ohms) & Cernox (K) 4th orde & er poly. \\
\hline Sample ID=W & Beam Energy $=1.1$ & $1 \mathrm{GeV}$ & on target $=135$ minut & ites & & & & \\
\hline post-irradiation coo & oldown data after 1. & $1 \mathrm{GeV}$ runs and aft & er 3-month room te & mperature anneal & & & & \\
\hline & & & & & & & & \\
\hline $5.000430 \mathrm{E}-04$ & $-2.375393 E-03$ & & & & $6 / 22 / 99$ 16:06:08 & & & \\
\hline$-5.001054 E-04$ & $2.351652 \mathrm{E}-03$ & & & & $6 / 22 / 99$ 16:06:11 & & & \\
\hline$-5.001052 E-04$ & $2.351676 \mathrm{E}-03$ & & & & $6 / 22 / 9916: 06: 13$ & & & \\
\hline $5.000432 E-04$ & $-2.375359 \mathrm{E}-03$ & & & & $6 / 22 / 99$ 16:06:16 & & & \\
\hline $5.000430 \mathrm{E}-04$ & $-2.375368 E-03$ & & & & $6 / 22 / 99$ 16:06:19 & & & \\
\hline$-5.001064 \mathrm{E}-04$ & $2.351687 \mathrm{E}-03$ & & & & $6 / 22 / 9916: 06: 22$ & & & \\
\hline$-5.001060 \mathrm{E}-04$ & $2.351688 \mathrm{E}-03$ & & & & $6 / 22 / 99$ 16:06:25 & & & \\
\hline $5.000425 \mathrm{E}-04$ & $-2.375367 \mathrm{E}-03$ & & & & $6 / 22 / 99$ 16:06:27 & & & \\
\hline $5.000435 E-04$ & $-2.375383 E-03$ & & & & $6 / 22 / 9916: 06: 30$ & & & \\
\hline$-5.001060 \mathrm{E}-04$ & $2.351679 \mathrm{E}-03$ & & & & $6 / 22 / 9916: 06: 33$ & & & \\
\hline$-5.001056 \mathrm{E}-04$ & $2.351690 \mathrm{E}-03$ & & & & $6 / 22 / 99$ 16:06:36 & & & \\
\hline $5.000434 \mathrm{E}-04$ & $-2.375335 E-03$ & & & & $6 / 22 / 99$ 16:06:39 & & & \\
\hline $5.000436 \mathrm{E}-04$ & $-2.375370 \mathrm{E}-03$ & & & & $6 / 22 / 9916: 06: 42$ & & & \\
\hline$-5.001058 \mathrm{E}-04$ & $2.351686 \mathrm{E}-03$ & & & & $6 / 22 / 9916: 06: 44$ & & & \\
\hline$-5.001057 \mathrm{E}-04$ & $2.351704 \mathrm{E}-03$ & & & & $6 / 22 / 99$ 16:06:47 & & & \\
\hline $5.000430 \mathrm{E}-04$ & $-2.375334 \mathrm{E}-03$ & & & & $6 / 22 / 9916: 06: 50$ & & & \\
\hline $5.000439 \mathrm{E}-04$ & $-2.375355 \mathrm{E}-03$ & & & & $6 / 22 / 9916: 06: 53$ & & & \\
\hline$-5.001064 \mathrm{E}-04$ & $2.351721 \mathrm{E}-03$ & & & & $6 / 22 / 9916: 06: 56$ & & & \\
\hline$-5.001058 E-04$ & $2.351700 \mathrm{E}-03$ & & & & $6 / 22 / 9916: 06: 59$ & & & \\
\hline $5.000432 E-04$ & $-2.375361 E-03$ & & & & $6 / 22 / 9916: 07: 01$ & & & \\
\hline 5.000440 E-04 & $-2.375344 \mathrm{E}-03$ & & & & $6 / 22 / 99 \quad 16: 07: 04$ & & & \\
\hline$-5.001063 E-04$ & $2.351683 E-03$ & & & & 6/22/99 16:07:07 & & & \\
\hline$-5.001061 E-04$ & $2.351718 \mathrm{E}-03$ & & & & $6 / 22 / 9916: 07: 10$ & & & \\
\hline 5.000430 E-04 & $-2.375342 \mathrm{E}-03$ & & & & $6 / 22 / 99$ 16:07:13 & & & \\
\hline $5.000432 \mathrm{E}-04$ & $-2.375373 \mathrm{E}-03$ & & & & $6 / 22 / 99$ 16:07:16 & & & \\
\hline$-5.001058 E-04$ & $2.351701 \mathrm{E}-03$ & & & & 6/22/99 16:07:18 & & & \\
\hline$-5.001058 E-04$ & $2.351705 E-03$ & & & & $6 / 22 / 99$ 16:07:21 & & & \\
\hline $5.000437 \mathrm{E}-04$ & $-2.375349 \mathrm{E}-03$ & & & & $6 / 22 / 9916: 07: 24$ & & & \\
\hline 5.000436 E-04 & $-2.375351 \mathrm{E}-03$ & & & & $6 / 22 / 99$ 16:07:27 & & & \\
\hline$-5.001056 \mathrm{E}-04$ & $2.351717 \mathrm{E}-03$ & & & & $6 / 22 / 9916: 07: 30$ & & & \\
\hline$-5.001056 \mathrm{E}-04$ & $2.351666 \mathrm{E}-03$ & & & & $6 / 22 / 99$ 16:07:33 & & & \\
\hline $5.000436 \mathrm{E}-04$ & $-2.375327 \mathrm{E}-03$ & & & & $6 / 22 / 99$ 16:07:35 & & & \\
\hline $5.000432 \mathrm{E}-04$ & $-2.375334 \mathrm{E}-03$ & & & & $6 / 22 / 99$ 16:07:38 & & & \\
\hline$-5.001056 \mathrm{E}-04$ & $2.351716 \mathrm{E}-03$ & & & & $6 / 22 / 9916: 07: 41$ & & & \\
\hline$-5.001057 \mathrm{E}-04$ & $2.351717 \mathrm{E}-03$ & & & & $6 / 22 / 99$ 16:07:44 & & & \\
\hline $5.000436 \mathrm{E}-04$ & $-2.375348 E-03$ & & & & $6 / 22 / 99$ 16:07:47 & & & \\
\hline $5.000437 \mathrm{E}-04$ & $-2.375336 \mathrm{E}-03$ & & & & $6 / 22 / 9916: 07: 49$ & & & \\
\hline$-5.001063 E-04$ & $2.351722 \mathrm{E}-03$ & & & & $6 / 22 / 99$ 16:07:52 & & & \\
\hline$-5.001063 E-04$ & $2.351723 \mathrm{E}-03$ & & & & $6 / 22 / 99$ 16:07:55 & & & \\
\hline $5.000430 \mathrm{E}-04$ & $-2.375354 \mathrm{E}-03$ & & & & $6 / 22 / 9916: 07: 58$ & & & \\
\hline $5.000746 E-04$ & $2.363526 \mathrm{E}-03$ & 4.726346 & $1.915638 \mathrm{E}-05$ & 1.181946E-06 & $6 / 22 / 9916: 08: 02$ & 4791.056318 & 4.828908413 & \\
\hline & & & & & & & & \\
\hline & & & & & & & & \\
\hline
\end{tabular}


TUNGSTEN.XIs

\begin{tabular}{|c|c|c|c|c|c|c|c|c|}
\hline Current (A) & Voltage (V) & Res.(ohms) & Std. Dev. Of $\Omega$ & Power (W) & Date/time & Cernox (ohms) & Cernox (K) 4th orde & er poly. \\
\hline Sample ID $=W$ & Beam Energy $=1.1$ & Time on & $n$ target $=135$ minut & tes & & & & \\
\hline post-irradiation cool & oldown data after 1.1 & $1 \mathrm{GeV}$ runs and afte & er 3-month room ten & mperature anneal & & & & \\
\hline & & & & & & & & \\
\hline $9.999841 \mathrm{E}-04$ & $-4.738236 \mathrm{E}-03$ & & & & 6/22/99 16:09:18 & & & \\
\hline$-1.000027 \mathrm{E}-03$ & $4.714446 \mathrm{E}-03$ & & & & 6/22/99 16:09:21 & & & \\
\hline$-1.000026 \mathrm{E}-03$ & 4.714450E-03 & & & & $6 / 22 / 9916: 09: 24$ & & & \\
\hline 9.999841E-04 & $-4.738243 E-03$ & & & & 6/22/99 16:09:27 & & & \\
\hline $9.999837 \mathrm{E}-04$ & $-4.738264 \mathrm{E}-03$ & & & & 6/22/99 16:09:30 & & & \\
\hline$-1.000028 \mathrm{E}-03$ & 4.714512E-03 & & & & $6 / 22 / 99$ 16:09:33 & & & \\
\hline$-1.000027 \mathrm{E}-03$ & 4.714470E-03 & & & & 6/22/99 16:09:35 & & & \\
\hline $9.999840 \mathrm{E}-04$ & $-4.738239 \mathrm{E}-03$ & & & & 6/22/99 16:09:38 & & & \\
\hline $9.999836 \mathrm{E}-04$ & $-4.738249 \mathrm{E}-03$ & & & & $6 / 22 / 99$ 16:09:41 & & & \\
\hline$-1000028 \mathrm{E}-03$ & 4.714509E-03 & & & & $6 / 22 / 99$ 16:09:44 & & & \\
\hline$-1.000027 \mathrm{E}-03$ & 4.714496E-03 & & & & $6 / 22 / 99$ 16:09:47 & & & \\
\hline $9.999842 \mathrm{E}-04$ & $-4.738213 E-03$ & & & & $6 / 22 / 9916: 09: 49$ & & & \\
\hline $9.999836 \mathrm{E}-04$ & $-4.738215 E-03$ & & & & $6 / 22 / 99$ 16:09:52 & & & \\
\hline$-1.000028 \mathrm{E}-03$ & $4.714528 \mathrm{E}-03$ & & & & $6 / 22 / 99$ 16:09:55 & & & \\
\hline$-1.000027 E-03$ & $4.714502 \mathrm{E}-03$ & & & & 6/22/99 16:09:58 & & & \\
\hline $9.999843 E-04$ & $-4.738203 E-03$ & & & & $6 / 22 / 99$ 16:10:01 & & & \\
\hline $9.999842 E-04$ & $-4.738219 \mathrm{E}-03$ & & & & $6 / 22 / 9916: 10: 04$ & & & \\
\hline$-1.000027 E-03$ & 4.714508E-03 & & & & 6/22/99 16:10:06 & & & \\
\hline$-1.000027 \mathrm{E}-03$ & 4.714512E-03 & & & & $6 / 22 / 99$ 16:10:09 & & & \\
\hline $9.999850 \mathrm{E}-04$ & $-4.738227 \mathrm{E}-03$ & & & & $6 / 22 / 99$ 16:10:12 & & & \\
\hline $9.999844 E-04$ & $-4.738240 \mathrm{E}-03$ & & & & $6 / 22 / 99 \quad 16: 10: 15$ & & & \\
\hline$-1.000028 \mathrm{E}-03$ & 4.714495E-03 & & & & $6 / 22 / 99$ 16:10:18 & & & \\
\hline$-1.000027 E-03$ & 4.714511E-03 & & & & $6 / 22 / 9916: 10: 21$ & & & \\
\hline $9.999846 \mathrm{E}-04$ & $-4.738241 E-03$ & & & & $6 / 22 / 9916: 10: 23$ & & & \\
\hline $9.999839 E-04$ & $-4.738239 \mathrm{E}-03$ & & & & $6 / 22 / 9916: 10: 26$ & & & \\
\hline$-1.000028 \mathrm{E}-03$ & $4.714490 \mathrm{E}-03$ & & & & $6 / 22 / 9916: 10: 29$ & & & \\
\hline$-1.000028 \mathrm{E}-03$ & 4.714512E-03 & & & & $6 / 22 / 99$ 16:10:32 & & & \\
\hline $9.999842 \mathrm{E}-04$ & $-4.738238 \mathrm{E}-03$ & & & & $6 / 22 / 9916: 10: 35$ & & & \\
\hline $9.999833 \mathrm{E}-04$ & $-4.738232 E-03$ & & & & $6 / 22 / 99$ 16:10:38 & & & \\
\hline$-1.000028 E-03$ & $4.714477 \mathrm{E}-03$ & & & & $6 / 22 / 99$ 16:10:40 & & & \\
\hline$-1.000028 E-03$ & $4.714482 \mathrm{E}-03$ & & & & $6 / 22 / 99$ 16:10:43 & & & \\
\hline $9.999835 E-04$ & $-4.738244 \mathrm{E}-03$ & & & & $6 / 22 / 99$ 16:10:46 & & & \\
\hline $9.999836 \mathrm{E}-04$ & $-4.738237 \mathrm{E}-03$ & & & & $6 / 22 / 99$ 16:10:49 & & & \\
\hline$-1.000029 \mathrm{E}-03$ & 4.714504E-03 & & & & $6 / 22 / 99$ 16:10:52 & & & \\
\hline$-1.000028 \mathrm{E}-03$ & $4.714526 \mathrm{E}-03$ & & & & $6 / 22 / 9916: 10: 54$ & & & \\
\hline $9.999843 \mathrm{E}-04$ & $-4.738220 \mathrm{E}-03$ & & & & $6 / 22 / 99$ 16:10:57 & & & \\
\hline 9.999837E-04 & $-4.738226 E-03$ & & & & $6 / 22 / 99$ 16:11:00 & & & \\
\hline$-1.000028 \mathrm{E}-03$ & $4.714498 \mathrm{E}-03$ & & & & $6 / 22 / 99$ 16:11:03 & & & \\
\hline$-1.000028 E-03$ & $4.714510 \mathrm{E}-03$ & & & & 6/22/99 16:11:06 & & & \\
\hline $9.999843 \mathrm{E}-04$ & $-4.738254 E-03$ & & & & 6/22/99 16:11:09 & & & \\
\hline $1.000006 \mathrm{E}-03$ & $4.726365 \mathrm{E}-03$ & 4.726338 & $1.101649 E-05$ & $4.726410 \mathrm{E}-06$ & $6 / 22 / 99$ 16:11:13 & 4790.650399 & 4.829139903 & \\
\hline & & & & & & & & \\
\hline
\end{tabular}


TUNGSTEN.XIS

\begin{tabular}{|c|c|c|c|c|c|c|c|c|}
\hline \multirow{2}{*}{\begin{tabular}{|l|} 
Current $(A)$ \\
Sample $I D=W$
\end{tabular}} & Voltage (V) & Res.(ohms) & Std. Dev. Of $\Omega$ & \multirow{2}{*}{$\frac{\text { Power }(W)}{\text { jtes }}$} & \multirow[t]{2}{*}{ Date/time } & \multirow[t]{2}{*}{ Cernox (ohms) } & \multicolumn{2}{|c|}{ Cernox (K) 4th order poly. } \\
\hline & Beam Energy $=1.1$ & Time ol & on target $=135 \mathrm{minu}$ & & & & & \\
\hline \multicolumn{9}{|c|}{ post-irradiation cooldown data after $1.1 \mathrm{GeV}$ runs and after 3 -month room temperature anneal } \\
\hline & & & & & & & & \\
\hline $2.000268 \mathrm{E}-03$ & $-9.465684 \mathrm{E}-03$ & & & & $6 / 22 / 9916: 12: 26$ & & & \\
\hline$-2.000833 E-03$ & 9.444249E-03 & & & & $6 / 22 / 9916: 12: 28$ & & & \\
\hline$-2.000829 \mathrm{E}-03$ & $9.444217 \mathrm{E}-03$ & & & & $6 / 22 / 99$ 16:12:31 & & & \\
\hline $2.000279 \mathrm{E}-03$ & $-9.465803 E-03$ & & & & $6 / 22 / 99$ 16:12:34 & & & \\
\hline $2.000277 \mathrm{E}-03$ & $-9.465745 \mathrm{E}-03$ & & & & 6/22/99 16:12:37 & & & \\
\hline$-2.000826 \mathrm{E}-03$ & $9.444135 \mathrm{E}-03$ & & & & $6 / 22 / 99$ 16:12:40 & & & \\
\hline$-2.000826 \mathrm{E}-03$ & $9.444216 \mathrm{E}-03$ & & & & $6 / 22 / 99$ 16:12:43 & & & \\
\hline $2.000283 E-03$ & $-9.465710 \mathrm{E}-03$ & & & & $6 / 22 / 99$ 16:12:45 & & & \\
\hline $2.000275 \mathrm{E}-03$ & $-9.465787 \mathrm{E}-03$ & & & & $6 / 22 / 9916: 12: 48$ & & & \\
\hline$-2.000825 E-03$ & 9.444185E-03 & & & & $6 / 22 / 9916: 12: 51$ & & & \\
\hline$-2.000829 E-03$ & 9.444253E-03 & & & & $6 / 22 / 99 \quad 16: 12: 54$ & & & \\
\hline $2.000282 E-03$ & $-9.465694 \mathrm{E}-03$ & & & & 6/22/99 16:12:57 & & & \\
\hline $2.000283 E-03$ & $-9.465758 \mathrm{E}-03$ & & & & $6 / 22 / 99$ 16:13:00 & & & \\
\hline$-2.000819 E-03$ & $9.444208 \mathrm{E}-03$ & & & & $6 / 22 / 9916: 13: 02$ & & & \\
\hline$-2.000818 E-03$ & $9.444302 \mathrm{E}-03$ & & & & $6 / 22 / 99$ 16:13:05 & & & \\
\hline $2.000279 E-03$ & $-9.465702 \mathrm{E}-03$ & & & & $6 / 22 / 9916: 13: 08$ & & & \\
\hline $2.000288 \mathrm{E}-03$ & $-9.465699 \mathrm{E}-03$ & & & & 6/22/99 16:13:11 & & & \\
\hline$-2.000821 \mathrm{E}-03$ & 9.444213E-03 & & & & $6 / 22 / 99$ 16:13:14 & & & \\
\hline$-2.000826 \mathrm{E}-03$ & $9.444167 \mathrm{E}-03$ & & & & 6/22/99 16:13:17 & & & \\
\hline $2.000281 \mathrm{E}-03$ & $-9.465715 \mathrm{E}-03$ & & & & $6 / 22 / 99$ 16:13:19 & & & \\
\hline $2.000282 \mathrm{E}-03$ & $-9.465763 \mathrm{E}-03$ & & & & $6 / 22 / 99$ 16:13:22 & & & \\
\hline$-2.000822 \mathrm{E}-03$ & $9.444279 \mathrm{E}-03$ & & & & $6 / 22 / 99$ 16:13:25 & & & \\
\hline$-2.000822 \mathrm{E}-03$ & $9.444239 \mathrm{E}-03$ & & & & $6 / 22 / 99$ 16:13:28 & & & \\
\hline $2.000284 \mathrm{E}-03$ & $-9.465719 \mathrm{E}-03$ & & & & $6 / 22 / 99$ 16:13:31 & & & \\
\hline $2.000283 E-03$ & $-9.465767 \mathrm{E}-03$ & & & & $6 / 22 / 99$ 16:13:34 & & & \\
\hline$-2.000816 \mathrm{E}-03$ & $9.444287 E-03$ & & & & $6 / 22 / 99$ 16:13:36 & & & \\
\hline$-2.000825 E-03$ & $9.444322 \mathrm{E}-03$ & & & & $6 / 22 / 99$ 16:13:39 & & & \\
\hline $2.000283 E-03$ & $-9.465715 \mathrm{E}-03$ & & & & $6 / 22 / 9916: 13: 42$ & & & \\
\hline $2.000283 E-03$ & $-9.465657 E-03$ & & & & $6 / 22 / 99$ 16:13:45 & & & \\
\hline$-2.000822 E-03$ & $9.444331 \mathrm{E}-03$ & & & & $6 / 22 / 99$ 16:13:48 & & & \\
\hline$-2.000822 E-03$ & 9.444283E-03 & & & & $6 / 22 / 99$ 16:13:51 & & & \\
\hline $2.000286 E-03$ & $-9.465810 \mathrm{E}-03$ & & & & $6 / 22 / 99$ 16:13:53 & & & \\
\hline $2.000286 \mathrm{E}-03$ & $-9.465775 \mathrm{E}-03$ & & & & $6 / 22 / 99$ 16:13:56 & & & \\
\hline$-2.000831 E-03$ & $9.444331 \mathrm{E}-03$ & & & & $6 / 22 / 99$ 16:13:59 & & & \\
\hline$-2.000830 \mathrm{E}-03$ & $9.444246 \mathrm{E}-03$ & & & & $6 / 22 / 99$ 16:14:02 & & & \\
\hline $2.000280 \mathrm{E}-03$ & $-9.465709 \mathrm{E}-03$ & & & & $6 / 22 / 99$ 16:14:05 & & & \\
\hline $2.000281 \mathrm{E}-03$ & $-9.465688 \mathrm{E}-03$ & & & & $6 / 22 / 9916: 14: 07$ & & & \\
\hline$-2.000820 \mathrm{E}-03$ & $9.444242 \mathrm{E}-03$ & & & & $6 / 22 / 99$ 16:14:10 & & & \\
\hline$-2.000828 \mathrm{E}-03$ & $9.444327 \mathrm{E}-03$ & & & & $6 / 22 / 9916: 14: 13$ & & & \\
\hline $2.000281 \mathrm{E}-03$ & $-9.465682 E-03$ & & & & $6 / 22 / 9916: 14: 16$ & & & \\
\hline $2.000553 \mathrm{E}-03$ & $9.454990 \mathrm{E}-03$ & 4.726189 & $1.554656 \mathrm{E}-05$ & $1.891525 \mathrm{E}-05$ & $6 / 22 / 9916: 14: 20$ & 4790.495053 & 4.829228504 & \\
\hline
\end{tabular}


